Supporting Information

\title{
Sulfur-Directed C7-Selective Alkenylation of Indoles under Rhodium Catalysis
}

Chandrababu Naidu Kona, ${ }^{a}$ Yuji Nishii, ${ }^{*}, \mathrm{~b}$ and Masahiro Miura ${ }^{*}$ a

${ }^{a}$ Innovative Catalysis Science Division, Institute for Open and Transdisciplinary Research

Initiatives (ICS-OTRI), Osaka University, Suita, Osaka 565-0871, Japan

b Department of Applied Chemistry, Graduate School of Engineering, Osaka University, Suita, Osaka 565-0871, Japan

y_nishii@chem.eng.osaka-u.ac.jp.

miura@chem.eng.osaka-u.ac.jp.

\section{Contents:}

1. General information $\quad$ S2

2. Additional data $\quad$ S3

3. Deuterium incorporation $\quad$ S5

4. Experimental procedures and identification data $\quad$ S7

$\begin{array}{ll}\text { 5. X-ray crystallographic analysis } & \text { S27 }\end{array}$

$\begin{array}{ll}\text { 6. Computational study } & \text { S30 }\end{array}$

$\begin{array}{ll}\text { 7. Copy of NMR spectra } & \text { S32 }\end{array}$

$\begin{array}{ll}\text { 8. References } & \text { S77 }\end{array}$ 


\section{General information}

All manipulations were performed under $\mathrm{N}_{2}$ using standard screw cap sealed tubes unless otherwise noted. Toluene, acetonitrile, $N, N$-dimethylformamide (DMF) and 1,4-dioxane were dried and deoxygenated by a Glass Counter Solvent Dispending System (Nikko Hansen \& Co., Ltd.). Dichloroethane (DCE) and $\mathrm{PhCF}_{3}$ were distilled from $\mathrm{CaH}_{2}$ and stored with MS 4A. THF and ethanol were purchased as dehydrated solvents and used as received. $\mathrm{Cp} * \mathrm{Rh}$ complexes were prepared according to the literature procedure. ${ }^{1}$ All other reagents were purchased from commercial suppliers and used without further purification.

NMR spectra were recorded on Bruker AVANCE III 400 spectrometer operating at $400 \mathrm{MHz}\left({ }^{1} \mathrm{H} \mathrm{NMR}\right)$ and at $100 \mathrm{MHz}\left({ }^{13} \mathrm{C}\right.$ NMR). The chemical shifts were reported in parts per million $(\delta)$ relative to the resonance of internal standard TMS $(0.00 \mathrm{ppm})$ or the residual solvent signals for $\mathrm{CDCl}_{3}\left(7.26 \mathrm{ppm}\right.$ for ${ }^{1} \mathrm{H}$ NMR, $\delta 77.0$ for ${ }^{13} \mathrm{C}$ NMR). The peak patterns are indicated as follows: s, singlet; $d$, doublet; dd, double doublet; t, triplet; m, multiplet; q, quartet; bs, broad singlet. The coupling constants, $J$, are reported in Hertz (Hz). High resolution mass spectra (HRMS) were recorded on Bruker micrOTOF II-H3 by APCI-TOF. Single crystal X-ray diffraction analyses were conducted with Rigaku XtaLAB Synergy diffractometer and HyPix-6000 detector. Melting points were measured with Mettler Toledo MP90. Silica gel column chromatography was performed using Wakosil@C-200 $(64-210 \mu \mathrm{m})$. Preparative gel permeation chromatography (GPC) was conducted with YMC T2000 column. 


\section{Additional data}

Table S1. Optimization study

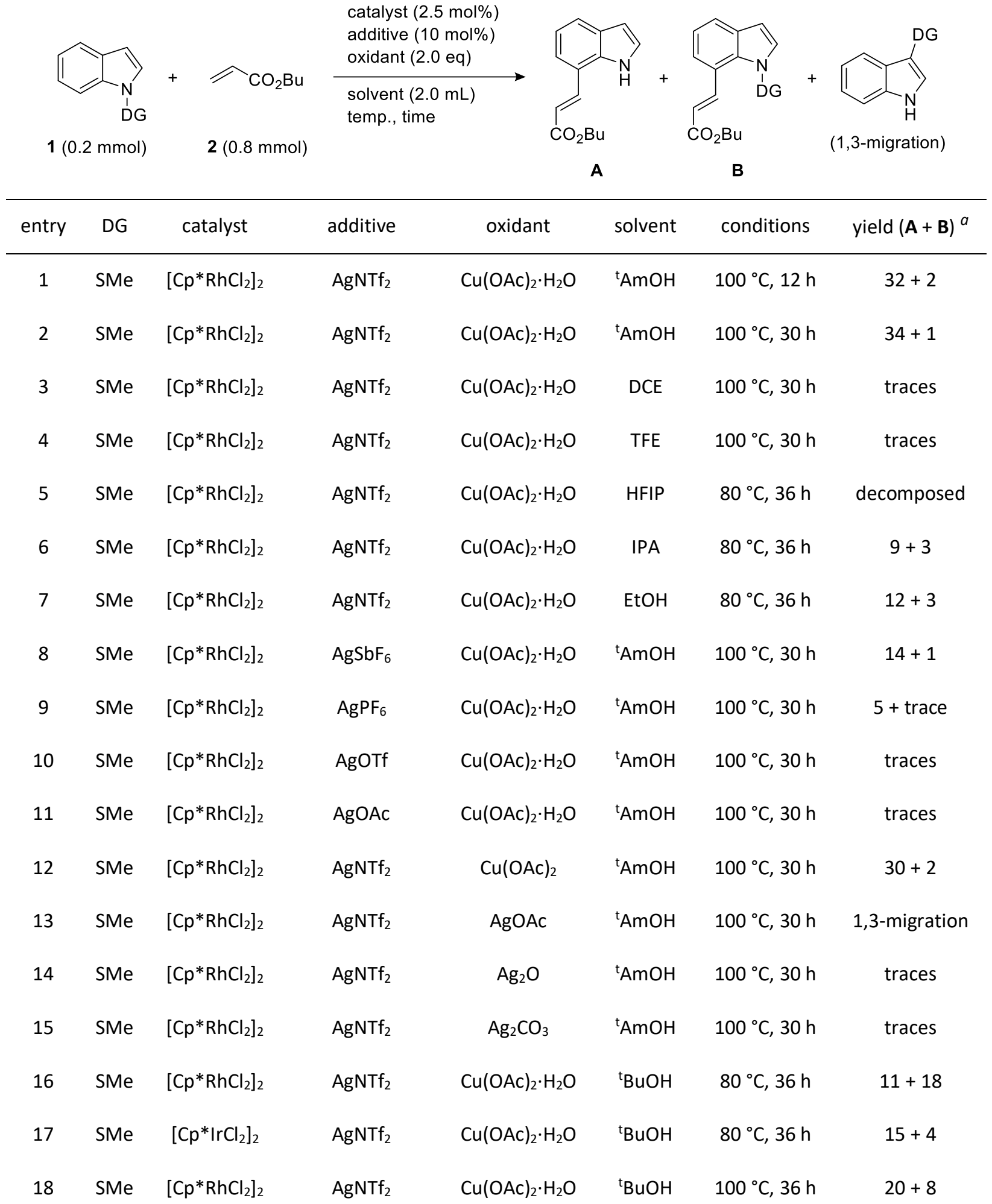


Table S1. Optimization study (continued)

\begin{tabular}{|c|c|c|c|c|c|c|c|}
\hline entry & DG & catalyst & additive & oxidant & solvent & conditions & yield $(\mathbf{A}+\mathbf{B})^{a}$ \\
\hline 19 & SMe & {$\left[\mathrm{Cp}^{*} \mathrm{RhCl}_{2}\right]_{2}$} & $\begin{array}{c}\mathrm{AgNTf}_{2} \\
\mathrm{AcOH}(0.5 \mathrm{~mL})\end{array}$ & $\mathrm{Cu}(\mathrm{OAc})_{2} \cdot \mathrm{H}_{2} \mathrm{O}$ & $\begin{array}{c}{ }^{\text {t}} \mathrm{BuOH} \\
(1.5 \mathrm{~mL})\end{array}$ & $100^{\circ} \mathrm{C}, 36 \mathrm{~h}$ & 1,3-migration \\
\hline 20 & SMe & {$\left[\mathrm{Cp} * \mathrm{RhCl}_{2}\right]_{2}$} & $\begin{array}{c}\mathrm{AgNTf}_{2} \\
\text { PivOH (1.0 eq) }\end{array}$ & $\mathrm{Cu}(\mathrm{OAc})_{2} \cdot \mathrm{H}_{2} \mathrm{O}$ & ${ }^{\mathrm{t}} \mathrm{BuOH}$ & $100^{\circ} \mathrm{C}, 36 \mathrm{~h}$ & $32+1$ \\
\hline $21^{b}$ & SMe & {$\left[\mathrm{Cp}^{*} \mathrm{RhCl}_{2}\right]_{2}$} & $\mathrm{AgNTf}_{2}$ & $\mathrm{Cu}(\mathrm{OAc})_{2} \cdot \mathrm{H}_{2} \mathrm{O}$ & ${ }^{\mathrm{t}} \mathrm{BuOH}$ & $100^{\circ} \mathrm{C}, 36 \mathrm{~h}$ & (41) \\
\hline $22^{b}$ & SMe & {$\left[\mathrm{Cp}^{*} \mathrm{RhCl}_{2}\right]_{2}$} & $\mathrm{AgNTf}_{2}$ & $\mathrm{Cu}(\mathrm{OAc})_{2} \cdot \mathrm{H}_{2} \mathrm{O}$ & ${ }^{\mathrm{t}} \mathrm{BuOH}$ & $90^{\circ} \mathrm{C}, 12 \mathrm{~h}$ & $(42)$ \\
\hline $23^{b}$ & SCy & {$\left[\mathrm{Cp} * \mathrm{RhCl}_{2}\right]_{2}$} & $\mathrm{AgNTf}_{2}$ & $\mathrm{Cu}(\mathrm{OAc})_{2} \cdot \mathrm{H}_{2} \mathrm{O}$ & ${ }^{\mathrm{t}} \mathrm{BuOH}$ & $90^{\circ} \mathrm{C}, 12 \mathrm{~h}$ & (93) \\
\hline $24^{c}$ & SCy & $\mathrm{Cp} * \mathrm{Co}(\mathrm{CO}) \mathrm{I}_{2}$ & $\mathrm{AgNTf}_{2}$ & $\mathrm{Cu}(\mathrm{OAc})_{2} \cdot \mathrm{H}_{2} \mathrm{O}$ & ${ }^{\mathrm{t}} \mathrm{BuOH}$ & $90^{\circ} \mathrm{C}, 7 \mathrm{~h}$ & no reaction \\
\hline $25^{b}$ & SPh & {$\left[\mathrm{Cp} * \mathrm{RhCl}_{2}\right]_{2}$} & $\mathrm{AgNTf}_{2}$ & $\mathrm{Cu}(\mathrm{OAc})_{2} \cdot \mathrm{H}_{2} \mathrm{O}$ & ${ }^{\mathrm{t}} \mathrm{BuOH}$ & $90{ }^{\circ} \mathrm{C}, 12 \mathrm{~h}$ & no reaction \\
\hline
\end{tabular}

[a] Determined by ${ }^{1} \mathrm{H}$ NMR yield. Isolated yield of $\mathrm{A}$ is shown in parentheses. [b] With 5.0 mol \% catalyst and $20 \mathrm{~mol} \%$ additive. [c] With $10 \mathrm{~mol} \%$ catalyst and $20 \mathrm{~mol} \%$ additive. 


\section{Deuterium incorporation}

To a $5 \mathrm{~mm}$ NMR tube were added $\mathrm{Cp} * \mathrm{Rh}(\mathrm{OAc})_{2}(24.7 \mathrm{mg}, 0.07 \mathrm{mmol}), \mathbf{1 b}(16.0 \mathrm{mg}, 0.07 \mathrm{mmol})$, and $\mathrm{MeOH}-d_{4}(0.5 \mathrm{~mL})$. The tube was heated at $70{ }^{\circ} \mathrm{C}$ with an oil bath and the reaction was monitored by ${ }^{1} \mathrm{H}$ NMR analyses. As an control experiment, another NMR tube without Rh complex was prepared and monitored similarly.

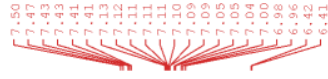

${ }^{1} \mathrm{H}$ NMR $\left(400 \mathrm{MHz}, \mathrm{CD}_{3} \mathrm{OD}\right)$ with $\mathrm{Cp} * \mathrm{Rh}(\mathrm{OAc})_{2}$ after $5 \mathrm{~h}$
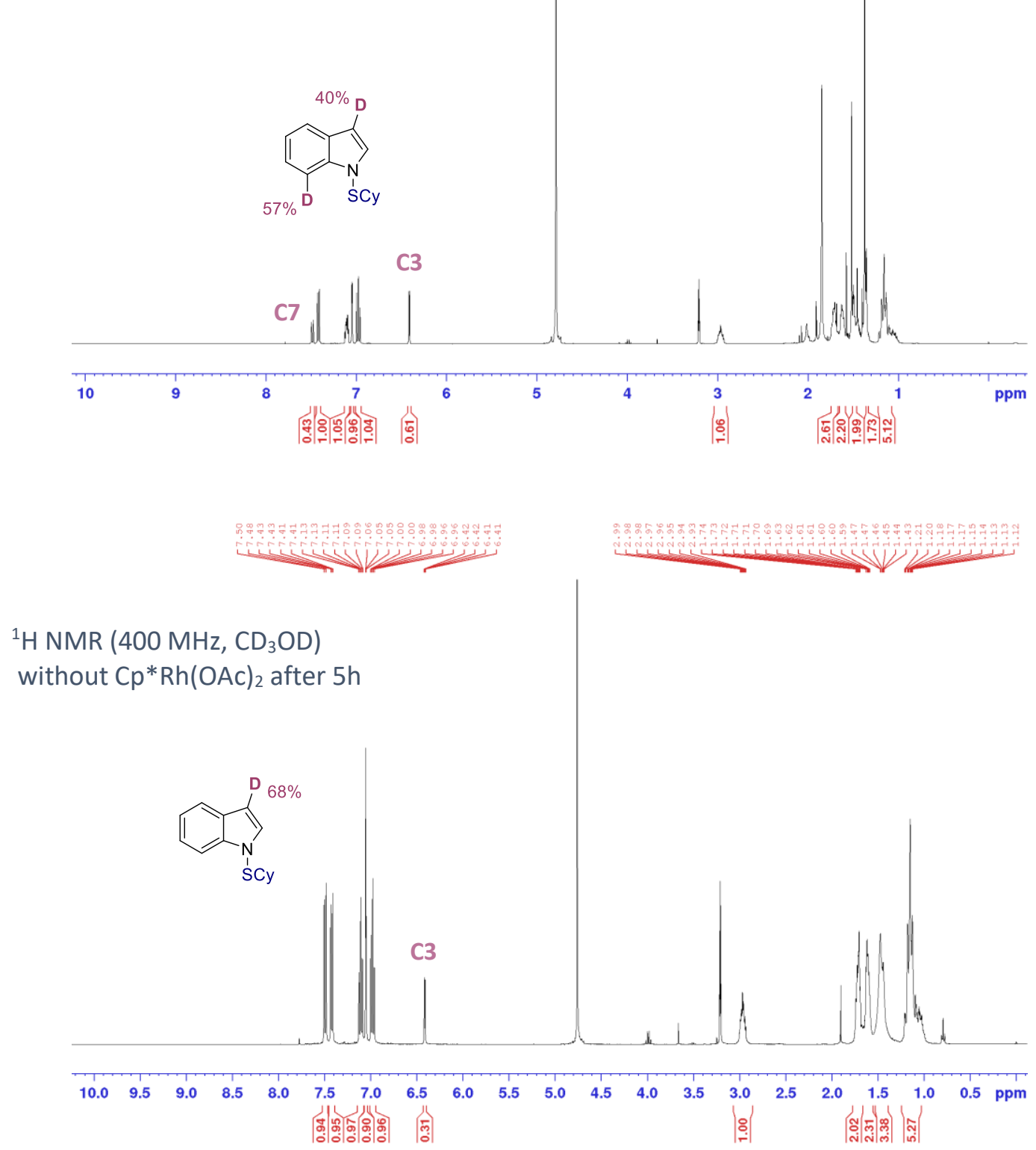


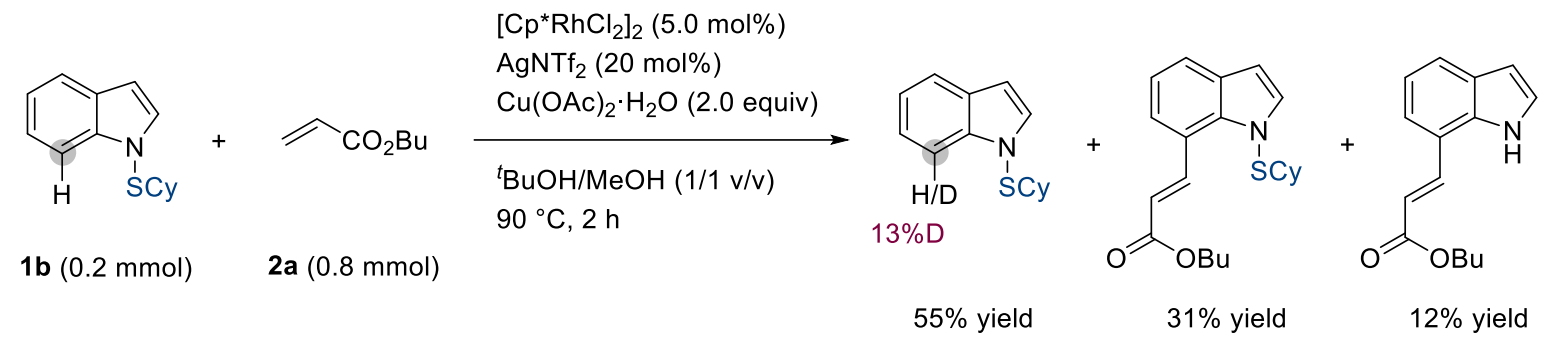

To an oven dried screw-top tube were added $N$-SCy-indole $\mathbf{1 b}(0.2 \mathrm{mmol}, 1.0$ equiv), olefin $\mathbf{2 a}(0.8 \mathrm{mmol}$, 4.0 equiv), $\left[\mathrm{Cp}^{*} \mathrm{RhCl}_{2}\right]_{2}(0.01 \mathrm{mmol}, 5.0 \mathrm{~mol} \%), \mathrm{AgNTf}_{2}\left(0.04 \mathrm{mmol}, 0.2\right.$ equiv), $\mathrm{Cu}(\mathrm{OAc})_{2} \cdot \mathrm{H}_{2} \mathrm{O}(0.4$ mmol, 2.0 equiv), $\mathrm{MeOH}-d_{4}(1.0 \mathrm{~mL})$, and ${ }^{t} \mathrm{BuOH}(1.0 \mathrm{~mL})$. The tube was flushed with $\mathrm{N}_{2}$ and sealed. The mixture was heated for $2 \mathrm{~h}$ at $90{ }^{\circ} \mathrm{C}$ with an oil bath. After cooling to room temperature, the resulting suspension was filtered through a pad of silica gel, and the filtrate was evaporated under reduced pressure to remove volatiles. The starting material was recovered by silica gel column chromatography (eluent: 5\% Ethyl acetate/Hexane). 


\section{Experimental procedures and identification data}

\section{4-1. General procedure for the synthesis of $N$-SCy-indoles 1}

$\mathrm{N}$-SCy-indoles 1 were prepared similarly to our previous report with slight modification. To a round-bottom flask were added indole (1.0 mmol), tetrabutylphosphonium bromide (34 mg, $0.1 \mathrm{mmol}$ ), aqueous $\mathrm{KOH}$ (1.0 mL, 50wt\%), and DCE (3.0 mL). At room temperature, a solution of $N$-SCy-phthalimide (287 mg, 1.1 $\mathrm{mmol})$ in DCE $(15 \mathrm{~mL})$ was added to the flask over 5 minutes with vigorous stirring, and another $0.1 \mathrm{mmol}$ (34 mg) of tetrabutylphosphonium bromide was added to the reaction mixture when the half amount of $N$ SCy-phthalimide solution was added. The resulting mixture was stirred until the complete consumption of indole was indicated by TLC followed by the addition of resin Amberlite ${ }^{\circledR}$ IRC120 H to neutralize the solution. The resin was filtered off through a pad of silica gel eluting with DCE. The filtrate was dried over $\mathrm{Na}_{2} \mathrm{SO}_{4}$ and concentrated under reduced pressure. The obtained crude material was purified by silica gel column chromatography (the eluent was indicated for each compound below).

1-(Cyclohexylthio)-1H-indole (1b)

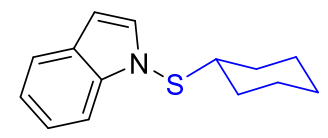

Prepared according to the general procedure in $10.0 \mathrm{mmol}$ scale.

Colorless liquid (2.2 g, 95\%); ( $\mathrm{R}_{f}=0.6,5 \%$ Ethyl acetate/Hexane); ${ }^{1} \mathrm{H}$ NMR $\left(400 \mathrm{MHz}, \mathrm{CDCl}_{3}\right) \delta 7.71$ (dq, $J=8.3,0.9 \mathrm{~Hz}, 1 \mathrm{H}), 7.65(\mathrm{dt}, J=7.8,0.9 \mathrm{~Hz}, 1 \mathrm{H}), 7.31-7.35(\mathrm{~m}, 1 \mathrm{H}), 7.19-7.23(\mathrm{~m}, 1 \mathrm{H}), 7.17(\mathrm{~d}, J=3.3$ $\mathrm{Hz}, 1 \mathrm{H}), 6.59$ (dd, $J=3.3,0.9 \mathrm{~Hz}, 1 \mathrm{H}), 3.08-3.15(\mathrm{~m}, 1 \mathrm{H}), 1.88-1.94(\mathrm{~m}, 2 \mathrm{H}), 1.77-1.82(\mathrm{~m}, 2 \mathrm{H}), 1.60-1.65$ (m, 1H), 1.15-1.41 (m, 5H); ${ }^{13} \mathrm{C}$ NMR (100 MHz, $\left.\mathrm{CDCl}_{3}\right) \delta 141.0,135.6,129.3,122.4,120.8,120.5,111.4$, 104.1, 50.8, 31.4, 25.7, 25.4; HRMS $m / z$ : $[\mathrm{M}+\mathrm{H}]^{+}$Calcd for $\mathrm{C}_{14} \mathrm{H}_{18} \mathrm{NS} 232.1154$; Found 232.1151.

1-(Phenylthio)-1H-indole (1c)

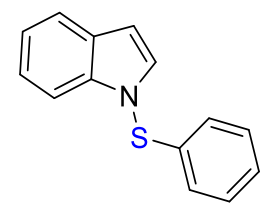

Prepared similarly to the general procedure using $N$-SPh-phthalimide.

White solid (290 mg, 86\%); $\left(\mathrm{R}_{f}=0.5\right.$, 5\% Ethyl acetate/Hexane); mp 132-134 ${ }^{\circ} \mathrm{C} ;{ }^{1} \mathrm{H}$ NMR (400 MHz, $\left.\mathrm{CDCl}_{3}\right) \delta$ 7.60-7.65 (m, 2H), 7.17-7.29 (m, 5H), 7.11-7.15 (m, 1H), 6.90-6.93 (m, 2H), $6.66(\mathrm{dd}, J=3.3$, $0.9 \mathrm{~Hz}, 1 \mathrm{H}) ;{ }^{13} \mathrm{C} \mathrm{NMR}\left(100 \mathrm{MHz}, \mathrm{CDCl}_{3}\right) \delta 140.2,138.9,134.2,129.5,129.2,126.7,123.8,123.1,121.2$, 121.1, 111.2, 105.8; HRMS m/z: $[\mathrm{M}+\mathrm{H}]^{+}$Calcd for $\mathrm{C}_{14} \mathrm{H}_{12} \mathrm{NS} 226.0685$; Found 226.0683. 
1-(Cyclohexylthio)-5-methyl-1 $H$-indole (1d)

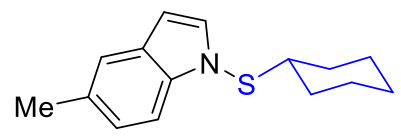

Colorless liquid (303 mg, 81\%); ( $\mathrm{R}_{f}=0.6,5 \%$ Ethyl acetate/Hexane); ${ }^{1} \mathrm{H}$ NMR (400 MHz, $\left.\mathrm{CDCl}_{3}\right) \delta 7.61$ $(\mathrm{d}, J=8.3 \mathrm{~Hz}, 1 \mathrm{H}), 7.45-7.45(\mathrm{~m}, 1 \mathrm{H}), 7.14-7.18(\mathrm{~m}, 2 \mathrm{H}), 6.52(\mathrm{dd}, J=3.2,0.9 \mathrm{~Hz}, 1 \mathrm{H}), 3.08-3.15(\mathrm{~m}$, $1 \mathrm{H}), 2.53(\mathrm{~s}, 3 \mathrm{H}), 1.89-1.94(\mathrm{~m}, 2 \mathrm{H}), 1.78-1.83(\mathrm{~m}, 2 \mathrm{H}), 1.62-1.67(\mathrm{~m}, 1 \mathrm{H}), 1.17-1.41(\mathrm{~m}, 5 \mathrm{H}) ;{ }^{13} \mathrm{C} \mathrm{NMR}$ $\left(100 \mathrm{MHz}, \mathrm{CDCl}_{3}\right) \delta 139.3,135.7,129.8,129.5,124.0,120.5,111.1,103.6,50.7,31.4,25.7,25.4,21.3$; HRMS $m / z:[\mathrm{M}+\mathrm{H}]^{+}$Calcd for $\mathrm{C}_{15} \mathrm{H}_{20} \mathrm{NS}$ 246.1311; Found 246.1310.

1-(Cyclohexylthio)-5-methoxy-1H-indole (1e)

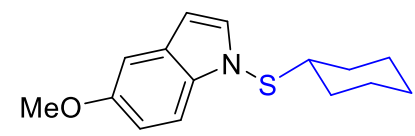

Colorless liquid (347 mg, 86\%); $\left(\mathrm{R}_{f}=0.6,5 \%\right.$ Ethyl acetate/Hexane); ${ }^{1} \mathrm{H}$ NMR $\left(400 \mathrm{MHz}, \mathrm{CDCl}_{3}\right) \delta 7.51$ $7.54(\mathrm{~m}, 1 \mathrm{H}), 7.09$ (d, $J=3.2 \mathrm{~Hz}, 1 \mathrm{H}), 7.05(\mathrm{~d}, J=2.4 \mathrm{~Hz}, 1 \mathrm{H}), 6.91$ (dd, $J=8.8,2.4 \mathrm{~Hz}, 1 \mathrm{H}), 6.46$ (dd, $J$ $=3.2,1.9 \mathrm{~Hz}, 1 \mathrm{H}), 3.85(\mathrm{~s}, 3 \mathrm{H}), 3.02-3.09(\mathrm{~m}, 1 \mathrm{H}), 1.83-1.88(\mathrm{~m}, 2 \mathrm{H}), 1,72-1.78(\mathrm{~m}, 2 \mathrm{H}), 1.56-1.61(\mathrm{~m}$, 1H), 1.12-1.35 (m, 5H); $\left.{ }^{13} \mathrm{C} \mathrm{NMR} \mathrm{(100} \mathrm{MHz,} \mathrm{CDCl}_{3}\right) \delta 154.8,136.3,136.0,129.7,112.4,112.2,103.8$, 102.7, 55.8, 50.6, 31.5, 25.7, 25.5; HRMS $m / z:[\mathrm{M}+\mathrm{H}]^{+}$Calcd for $\mathrm{C}_{15} \mathrm{H}_{20} \mathrm{NOS}$ 262.1260; Found 262.1252. 5-Chloro-1-(cyclohexylthio)-1H-indole (1f)

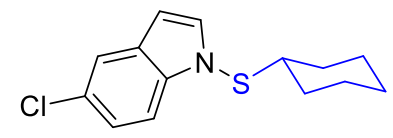

Colorless gum (343 mg, 85\%); ( $\mathrm{R}_{f}=0.6,5 \%$ Ethyl acetate/Hexane); ${ }^{1} \mathrm{H}$ NMR (400 MHz, $\left.\mathrm{CDCl}_{3}\right) \delta 7.54-$ $7.56(\mathrm{~m}, 2 \mathrm{H}), 7.21(\mathrm{dd}, J=8.7,1.9 \mathrm{~Hz}, 1 \mathrm{H}), 7.13(\mathrm{~d}, J=3.2 \mathrm{~Hz}, 1 \mathrm{H}), 6.47(\mathrm{dd}, J=3.3,0.8 \mathrm{~Hz}, 1 \mathrm{H}), 3.02-$ $3.10(\mathrm{~m}, 1 \mathrm{H}), 1.73-1.86(\mathrm{~m}, 4 \mathrm{H}), 1.57-1.62(\mathrm{~m}, 1 \mathrm{H}), 1.13-1.33(\mathrm{~m}, 5 \mathrm{H}) ;{ }^{13} \mathrm{C} \mathrm{NMR}\left(100 \mathrm{MHz}, \mathrm{CDCl}_{3}\right) \delta$ $139.5,136.9,130.3,126.3,122.8,120.2$, 112.6, 103.7, 51.0, 31.4, 25.7, 25.4; HRMS m/z: $[\mathrm{M}+\mathrm{H}]^{+}$Calcd for $\mathrm{C}_{14} \mathrm{H}_{17} \mathrm{ClNS} 266.0765$; Found 266.0758.

5-Bromo-1-(cyclohexylthio)-1H-indole (1g)

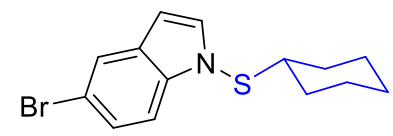

Colorless liquid (361 mg, 76\%); $\left(\mathrm{R}_{f}=0.6,5 \%\right.$ Ethyl acetate/Hexane); ${ }^{1} \mathrm{H}$ NMR $\left(400 \mathrm{MHz}, \mathrm{CDCl}_{3}\right) \delta 7.73$ $7.74(\mathrm{~m}, 1 \mathrm{H}), 7.53(\mathrm{dq}, J=8.8,0.8 \mathrm{~Hz}, 1 \mathrm{H}), 7.36(\mathrm{dd}, J=8.7,1.9 \mathrm{~Hz}, 1 \mathrm{H}), 7.13(\mathrm{~d}, J=3.3 \mathrm{~Hz}, 1 \mathrm{H}), 6.49$ 
$(\mathrm{dd}, J=3.3,0.9 \mathrm{~Hz}, 1 \mathrm{H}), 3.03-3.11(\mathrm{~m}, 1 \mathrm{H}), 1.73-1.87(\mathrm{~m}, 4 \mathrm{H}), 1.57-1.63(\mathrm{~m}, 1 \mathrm{H}), 1.12-1.34(\mathrm{~m}, 5 \mathrm{H}) ;{ }^{13} \mathrm{C}$ NMR (100 MHz, $\left.\mathrm{CDCl}_{3}\right) \delta 139.8,136.8,130.9,125.3,123.3,113.9,113.0,103.6,51.0,31.4,25.7,25.4$; HRMS m/z: $[\mathrm{M}+\mathrm{H}]^{+}$Calcd for $\mathrm{C}_{14} \mathrm{H}_{17} \mathrm{BrNS}$ 310.0260; Found 310.0267, 312.0248.

6-Chloro-1-(cyclohexylthio)-1 $H$-indole (1h)

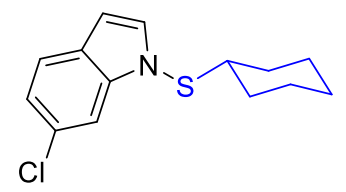

Colorless liquid (351 mg, 87\%); $\left(\mathrm{R}_{f}=0.6,5 \%\right.$ Ethyl acetate/Hexane); ${ }^{1} \mathrm{H}$ NMR $\left(400 \mathrm{MHz}, \mathrm{CDCl}_{3}\right) \delta 7.63$ $7.64(\mathrm{~m}, 1 \mathrm{H}), 7.48(\mathrm{~d}, J=8.3 \mathrm{~Hz}, 1 \mathrm{H}), 7.10-7.13(\mathrm{~m}, 2 \mathrm{H}), 6.50(\mathrm{dd}, J=3.3,0.9 \mathrm{~Hz}, 1 \mathrm{H}), 3.05-3.12(\mathrm{~m}, 1 \mathrm{H})$, 1.82-1.87 (m, 2H), 1.74-1.79 (m, 2H), 1.58-1.63 (m, 1H), 1.18-1.35 (m, 5H); $\left.{ }^{13} \mathrm{C} \mathrm{NMR} \mathrm{(100} \mathrm{MHz,} \mathrm{CDCl}_{3}\right)$ $\delta$ 141.6, 136.3, 128.7, 127.8, 121.6, 121.3, 111.6, 104.2, 50.8, 31.4, 25.7, 25.4; HRMS $m / z:[\mathrm{M}+\mathrm{H}]^{+}$Calcd for $\mathrm{C}_{14} \mathrm{H}_{17} \mathrm{ClNS} 266.0765$; Found 266.0765.

6-Bromo-1-(cyclohexylthio)-1H-indole (1i)

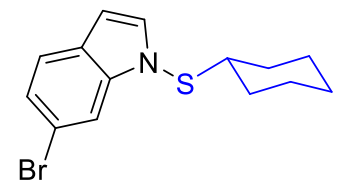

Colorless liquid (441 mg, 95\%); $\left(\mathrm{R}_{f}=0.6,5 \%\right.$ Ethyl acetate/Hexane); ${ }^{1} \mathrm{H}$ NMR $\left(400 \mathrm{MHz}, \mathrm{CDCl}_{3}\right) \delta 7.79$ $7.80(\mathrm{~m}, 1 \mathrm{H}), 7.44(\mathrm{~d}, J=8.3 \mathrm{~Hz}, 1 \mathrm{H}), 7.23-7.26(\mathrm{~m}, 1 \mathrm{H}), 7.09$ (d, $J=3.3 \mathrm{~Hz}, 1 \mathrm{H}), 6.50$ (dd, $J=3.3,0.9$ $\mathrm{Hz}, 1 \mathrm{H}), 3.05-3.13(\mathrm{~m}, 1 \mathrm{H}), 1.82-1.86(\mathrm{~m}, 2 \mathrm{H}), 1.74-1.79(\mathrm{~m}, 2 \mathrm{H}), 1.58-1.62(\mathrm{~m}, 1 \mathrm{H}), 1.18-1.34(\mathrm{~m}, 5 \mathrm{H})$; ${ }^{13} \mathrm{C}$ NMR $\left(100 \mathrm{MHz}, \mathrm{CDCl}_{3}\right) \delta 141.9,136.1,128.1,123.8,121.9,116.3,114.4,104.1,50.7,31.3,25.6$, 25.4; HRMS $m / z:[\mathrm{M}+\mathrm{H}]^{+}$Calcd for $\mathrm{C}_{14} \mathrm{H}_{17} \mathrm{BrNS}$ 310.0260; Found 310.0265.

Methyl 1-(cyclohexylthio)-1 $H$-indole-6-carboxylate (1j)

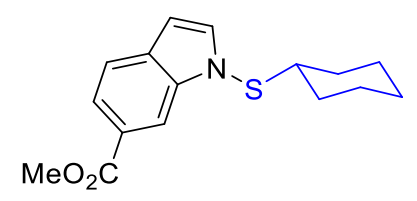

Colorless liquid (398 mg, 92\%); ( $\mathrm{R}_{f}=0.5,15 \%$ Ethyl acetate/Hexane); ${ }^{1} \mathrm{H}$ NMR $\left(400 \mathrm{MHz}, \mathrm{CDCl}_{3}\right) \delta 8.36$ $8.37(\mathrm{~m}, 1 \mathrm{H}), 7.84(\mathrm{dd}, J=8.3,1.5 \mathrm{~Hz}, 1 \mathrm{H}), 7.61(\mathrm{dd}, J=8.3,0.5 \mathrm{~Hz}, 1 \mathrm{H}), 7.27(\mathrm{~d}, J=3.2 \mathrm{~Hz}, 1 \mathrm{H}), 6.58$ $(\mathrm{dd}, J=3.2,0.9 \mathrm{~Hz}, 1 \mathrm{H}), 3.96(\mathrm{~s}, 3 \mathrm{H}), 3.08-3.15(\mathrm{~m}, 1 \mathrm{H}), 1.82-1.87(\mathrm{~m}, 2 \mathrm{H}), 1.73-1.78(\mathrm{~m}, 2 \mathrm{H}), 1.57-1.62$ $(\mathrm{m}, 1 \mathrm{H}), 1.15-1.35(\mathrm{~m}, 5 \mathrm{H}) ;{ }^{13} \mathrm{C} \mathrm{NMR}\left(100 \mathrm{MHz}, \mathrm{CDCl}_{3}\right) \delta 168.0,140.5,138.8,133.0,124.4,121.7,120.5$, 113.7, 104.4, 52.0, 50.9, 31.3, 25.6, 25.4; HRMS m/z: $[\mathrm{M}+\mathrm{H}]^{+}$Calcd for $\mathrm{C}_{16} \mathrm{H}_{20} \mathrm{NO}_{2} \mathrm{~S}$ 290.1209; Found 290.1204. 
1-(Cyclohexylthio)-3-methyl-1H-indole (1k)

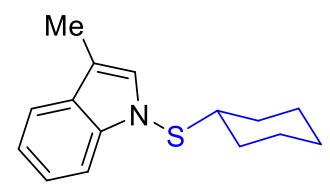

Colorless liquid (348 mg, 93\%); ( $\mathrm{R}_{f}=0.6,5 \%$ Ethyl acetate/Hexane); ${ }^{1} \mathrm{H}$ NMR (400 MHz, $\left.\mathrm{CDCl}_{3}\right) \delta 7.61$ (dt, $J=8.2,0.8 \mathrm{~Hz}, 1 \mathrm{H}), 7.52-7.54(\mathrm{~m}, 1 \mathrm{H}), 7.24-7.29(\mathrm{~m}, 1 \mathrm{H}), 7.14-7.18(\mathrm{~m}, 1 \mathrm{H}), 6.88(\mathrm{q}, J=1.2 \mathrm{~Hz}, 1 \mathrm{H})$, 3.00-3.07 (m, 1H), $2.29(\mathrm{~s}, 3 \mathrm{H}), 1.84-1.89(\mathrm{~m}, 2 \mathrm{H}), 1,72-1.77(\mathrm{~m}, 2 \mathrm{H}), 1.57-1.61(\mathrm{~m}, 1 \mathrm{H}), 1.14-1.36(\mathrm{~m}$, $5 \mathrm{H}) ;{ }^{13} \mathrm{C}$ NMR (100 MHz, $\left.\mathrm{CDCl}_{3}\right) \delta 141.4,132.8,129.9,122.4,120.1,118.8,113.1,111.4,50.8,31.5,25.8$, 25.5, 9.6; HRMS m/z: [M+H] ${ }^{+}$Calcd for $\mathrm{C}_{15} \mathrm{H}_{20} \mathrm{NS} 246.1311$; Found 246.1300.

1-(Cyclohexylthio)-2-methyl-1H-indole (11)

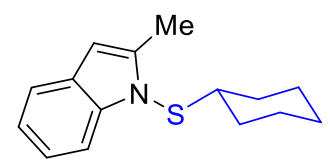

Brown solid (333 mg, 89\%); $\left(\mathrm{R}_{f}=0.5,5 \%\right.$ Ethyl acetate/Hexane); mp 67-69 ${ }^{\circ} \mathrm{C} ;{ }^{1} \mathrm{H} \mathrm{NMR}\left(400 \mathrm{MHz}, \mathrm{CDCl}_{3}\right)$ $\delta 7.83(\mathrm{~d}, J=8.2 \mathrm{~Hz}, 1 \mathrm{H}), 7.69(\mathrm{~d}, J=7.7 \mathrm{~Hz}, 1 \mathrm{H}), 7.39-7.43(\mathrm{~m}, 1 \mathrm{H}), 7.31-7.35(\mathrm{~m}, 1 \mathrm{H}), 6.54-6.55(\mathrm{~m}$, 1H), 3.24-3.32 (m, 1H), 2.69 (s, 3H), 1.89-2.03 (m, 4H), 1.76-1.79 (m, 1H), 1.48-1.57 (m, 2H), 1.34-1.44 $(\mathrm{m}, 3 \mathrm{H}) ;{ }^{13} \mathrm{C}$ NMR $\left(100 \mathrm{MHz}, \mathrm{CDCl}_{3}\right) \delta 141.7,141.6,129.0,121.3,120.6,119.5,111.3,102.6,50.5,31.1$, 25.6, 25.5, 13.4; HRMS m/z: $[\mathrm{M}+\mathrm{H}]^{+}$Calcd for $\mathrm{C}_{15} \mathrm{H}_{20} \mathrm{NS}$ 246.1311; Found 246.1297.

1-(Cyclohexylthio)-2-phenyl-1H-indole (1m)

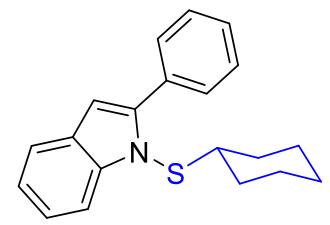

Colorless gum (435 mg, 94\%); ( $\mathrm{R}_{f}=0.5,5 \%$ Ethyl acetate/Hexane); ${ }^{1} \mathrm{H}$ NMR (400 MHz, $\left.\mathrm{CDCl}_{3}\right) \delta 7.75$ $7.78(\mathrm{~m}, 1 \mathrm{H}), 7.64-7.67(\mathrm{~m}, 2 \mathrm{H}), 7.61(\mathrm{~d}, J=7.8 \mathrm{~Hz}, 1 \mathrm{H}), 7.44-7.49(\mathrm{~m}, 2 \mathrm{H}), 7.39-7.43(\mathrm{~m}, 1 \mathrm{H}), 7.28-7.33$ (m, 1H), 7.18-7.22 (m, 1H), 6.69 (s, 1H), 2.85-2.93 (m, 1H), 1.46-1.56 (m, 5H), $1.06(\mathrm{bs}, 5 \mathrm{H}) ;{ }^{13} \mathrm{C}$ NMR $\left(100 \mathrm{MHz}, \mathrm{CDCl}_{3}\right) \delta 145.6,142.8,132.6,130.1,129.0,127.9,127.8,122.6,121.2,120.4,112.4,105.0$, 50.5, 30.7, 25.5, 25.3; HRMS $m / z:[\mathrm{M}+\mathrm{H}]^{+}$Calcd for $\mathrm{C}_{20} \mathrm{H}_{22} \mathrm{NS}$ 308.1467; Found 308.1464. 
1-(Cyclohexylthio)-2-(naphthalen-2-yl)-1H-indole (1n)

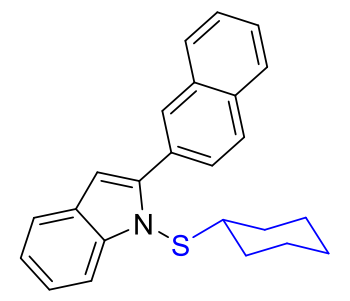

Pale red semisolid (338 mg, 92\%); $\left(\mathrm{R}_{f}=0.5,5 \%\right.$ Ethyl acetate/Hexane); ${ }^{1} \mathrm{H}$ NMR (400 MHz, $\left.\mathrm{CDCl}_{3}\right) \delta$ 8.14-8.15 (m, 1H), 7.88-7.96 (m, 3H), 7.77-7.80 (m, 2H), 7.61-7.64 (m, 1H), 7.51-7.56 (m, 2H), 7.30-7.34 (m, 1H), 7.19-7.23 (m, 1H), $6.79(\mathrm{~d}, J=0.7 \mathrm{~Hz}, 1 \mathrm{H}), 2.86-2.93(\mathrm{~m}, 1 \mathrm{H}), 1.40-1.52(\mathrm{~m}, 5 \mathrm{H}), 1.00-1.05(\mathrm{~m}$, $5 \mathrm{H}) ;{ }^{13} \mathrm{C} \mathrm{NMR}\left(100 \mathrm{MHz}, \mathrm{CDCl}_{3}\right) \delta 145.5,143.0,133.0,132.7,130.1,128.98,128.97,128.3,127.9,127.7$, 127.3, 126.2, 126.1, 122.6, 121.2, 120.5, 112.4, 105.5, 50.5, 30.7, 25.3 (1C alkyl peak overlapped); HRMS $m / z:[\mathrm{M}+\mathrm{H}]^{+}$Calcd for $\mathrm{C}_{24} \mathrm{H}_{24} \mathrm{NS} 358.1624$; Found 358.1627 .

4-(Cyclohexylthio)-1,2,3,4-tetrahydrocyclopenta[b]indole (10)

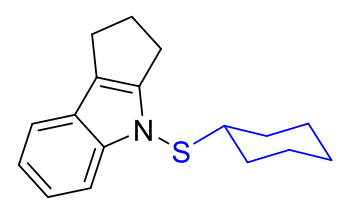

White solid (381 mg, 92\%); ( $\mathrm{R}_{f}=0.5,5 \%$ Ethyl acetate/Hexane); mp 99-101 ${ }^{\circ} \mathrm{C} ;{ }^{1} \mathrm{H}$ NMR (400 MHz, $\left.\mathrm{CDCl}_{3}\right) \delta$ 7.57-7.60 (m, 1H), 7.38-7.41 (m, 1H), 7.10-7.20 (m, 2H), 3.01-3.09 (m, 1H), 2.89-2.94 (m, 2H), 2.83-2.88 (m, 2H), 2.48-2.55 (m, 2H), 1.85-1.90 (m, 2H), 1.73-1.79 (m, 2H), 1.57-1.62 (m, 1H), 1.16-1.37 $(\mathrm{m}, 5 \mathrm{H}) ;{ }^{13} \mathrm{C} \mathrm{NMR}\left(100 \mathrm{MHz}, \mathrm{CDCl}_{3}\right) \delta 150.2,146.1,126.0,120.9,120.6,120.5,118.4,111.7,50.8,31.4$, 27.6, 25.9, 25.8, 25.5, 25.1; HRMS $m / z:[\mathrm{M}+\mathrm{H}]^{+}$Calcd for $\mathrm{C}_{17} \mathrm{H}_{22} \mathrm{NS}$ 272.1467; Found 272.1465.

9-(Cyclohexylthio)-2,3,4,9-tetrahydro-1 $H$-carbazole (1p)

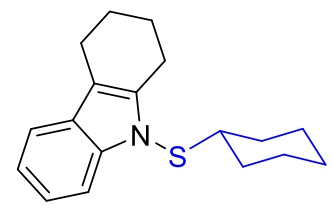

Colorless gum (397 mg, 93\%); ( $\mathrm{R}_{f}=0.5,5 \%$ Ethyl acetate/Hexane); ${ }^{1} \mathrm{H}$ NMR (400 MHz, $\left.\mathrm{CDCl}_{3}\right) \delta 7.65$ (dd, $J=8.1,0.8 \mathrm{~Hz}, 1 \mathrm{H}), 7.45-7.47(\mathrm{~m}, 1 \mathrm{H}), 7.21-7.25(\mathrm{~m}, 1 \mathrm{H}), 7.13-7.17(\mathrm{~m}, 1 \mathrm{H}), 3.04-3.11(\mathrm{~m}, 1 \mathrm{H})$, 2.71-2.86 (m, 4H), 1.85-1.98 (m, 6H), 1.75-1.81 (m, 2H), 1.60-1.65 (m, 1H), 1.20-1.41 (m, 5H); ${ }^{13} \mathrm{C}$ NMR $\left(100 \mathrm{MHz}, \mathrm{CDCl}_{3}\right) \delta 141.4,140.3,128.7,121.4,120.2,117.5,112.3,111.0,50.7,31.3,25.8,25.6,23.3$, 23.1, 22.9, 21.2; HRMS m/z: [M+H] $]^{+}$Calcd for $\mathrm{C}_{18} \mathrm{H}_{24} \mathrm{NS}$ 286.1624; Found 286.1616. 
9-(Cyclohexylthio)-9H-carbazole (1q)

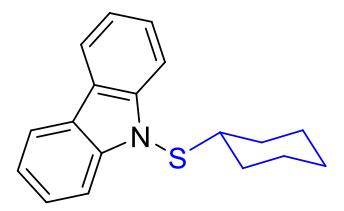

Colorless gum (404 mg, 96\%); ( $\mathrm{R}_{f}=0.5,5 \%$ Ethyl acetate/Hexane); ${ }^{1} \mathrm{H}$ NMR (400 MHz, $\left.\mathrm{CDCl}_{3}\right) \delta 8.03$ $8.05(\mathrm{~m}, 2 \mathrm{H}), 7.78-7.81(\mathrm{~m}, 2 \mathrm{H}), 7.48-7.52(\mathrm{~m}, 2 \mathrm{H}), 7.27-7.31(\mathrm{~m}, 2 \mathrm{H}), 3.17-3.25(\mathrm{~m}, 1 \mathrm{H}), 1.85-1.91(\mathrm{~m}$, $2 \mathrm{H}), 1.71-1.77(\mathrm{~m}, 2 \mathrm{H}), 1.55-1.60(\mathrm{~m}, 1 \mathrm{H}), 1.35-1.44(\mathrm{~m}, 2 \mathrm{H}), 1.15-1.28(\mathrm{~m}, 3 \mathrm{H}) ;{ }^{13} \mathrm{C}$ NMR $(100 \mathrm{MHz}$, $\left.\mathrm{CDCl}_{3}\right) \delta 144.5,126.1,124.2,120.6,120.0,111.3,50.3,31.7,25.8,25.5 ; \mathrm{HRMS} m / z:[\mathrm{M}+\mathrm{H}]^{+}$Calcd for $\mathrm{C}_{18} \mathrm{H}_{20} \mathrm{NS} 282.1311$; Found 282.1314. 


\section{4-2. Rhodium-catalyzed C7 alkenylation of indoles (Scheme 3 and 4)}

To an oven dried screw-top tube were added $N$-SCy-indole 1 ( $0.2 \mathrm{mmol}, 1.0$ equiv), olefin 2 ( $0.8 \mathrm{mmol}$, 4.0 equiv), $\left[\mathrm{Cp}^{*} \mathrm{RhCl}_{2}\right]_{2}(0.01 \mathrm{mmol}, 5.0 \mathrm{~mol} \%), \mathrm{AgNTf}_{2}\left(0.04 \mathrm{mmol}, 0.2\right.$ equiv), $\mathrm{Cu}(\mathrm{OAc})_{2} \cdot \mathrm{H}_{2} \mathrm{O}(0.4$ mmol, 2.0 equiv), and ${ }^{t} \mathrm{BuOH}(2.0 \mathrm{~mL})$. The tube was flushed with $\mathrm{N}_{2}$ and sealed. The mixture was heated for $12 \mathrm{~h}$ at $90{ }^{\circ} \mathrm{C}$ with an oil bath. After cooling to room temperature, the resulting suspension was filtered through a pad of silica gel, and the filtrate was evaporated under reduced pressure to remove volatiles. The obtained crude product was dissolved in THF $(5.0 \mathrm{~mL})$ and added tetrabutyl ammonium fluoride $(0.3 \mathrm{~mL}$ of 1.0 M THF solution, 1.5 equiv.). After stirring at room temperature for $1 \mathrm{~h}$, the mixture was poured into water and extracted with EtOAc. The organic layer was dried over $\mathrm{Na}_{2} \mathrm{SO}_{4}$, concentrated in vacuo, and purified by silica gel column chromatography (the eluent was indicated for each compounds below).

\section{4-3. Scale-up for the synthesis of 3ba (Scheme 3)}

To an oven dried screw-top tube were added $N$-SCy-indole $\mathbf{1 b}$ (233 mg, $1.0 \mathrm{mmol}$ ), olefin $\mathbf{2 a}$ (516 mg, 4.0 mmol), [Cp* $\left.\mathrm{RhCl}_{2}\right]_{2}(31 \mathrm{mg}, 0.05 \mathrm{mmol}), \mathrm{AgNTf}_{2}(78 \mathrm{mg}, 0.2 \mathrm{mmol}), \mathrm{Cu}(\mathrm{OAc})_{2} \cdot \mathrm{H}_{2} \mathrm{O}(402 \mathrm{mg}, 2.0 \mathrm{mmol})$, and ${ }^{t} \mathrm{BuOH}(10 \mathrm{~mL})$. The tube was flushed with $\mathrm{N}_{2}$ and sealed. The mixture was heated for $12 \mathrm{~h}$ at $90{ }^{\circ} \mathrm{C}$ with an oil bath. After cooling to room temperature, the resulting suspension was filtered through a pad of silica gel, and the filtrate was evaporated under reduced pressure to remove volatiles. The obtained crude product was dissolved in THF $(15 \mathrm{~mL})$ and added tetrabutyl ammonium fluoride $(1.5 \mathrm{~mL}$ of $1.0 \mathrm{M}$ THF solution). After stirring at room temperature for $1 \mathrm{~h}$, the mixture was poured into water and extracted with EtOAc. The organic layer was dried over $\mathrm{Na}_{2} \mathrm{SO}_{4}$, concentrated in vacuo, and purified by silica gel column chromatography to give $3 \mathbf{b a}(210 \mathrm{mg}, 86 \%)$.

Butyl (E)-3-(1H-indol-7-yl)acrylate (3ba)

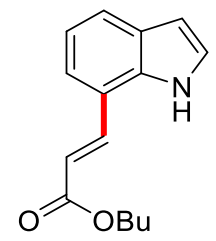

White solid (46 mg, 93\%); $\left(\mathrm{R}_{f}=0.4,20 \%\right.$ Ethyl acetate/Hexane); $\mathrm{mp} 74-76{ }^{\circ} \mathrm{C} ;{ }^{1} \mathrm{H} \mathrm{NMR}\left(400 \mathrm{MHz}, \mathrm{CDCl}_{3}\right)$ $\delta 9.05$ (bs, 1H), $8.14(\mathrm{~d}, J=16.0 \mathrm{~Hz}, 1 \mathrm{H}), 7.72-7.74(\mathrm{~m}, 1 \mathrm{H}), 7.46(\mathrm{~d}, J=7.4 \mathrm{~Hz}, 1 \mathrm{H}), 7.23-7.35(\mathrm{~m}, 1 \mathrm{H})$, $7.17(\mathrm{t}, J=7.6 \mathrm{~Hz}, 1 \mathrm{H}), 6.62(\mathrm{dd}, J=3.2,2.0 \mathrm{~Hz}, 1 \mathrm{H}), 6.60(\mathrm{~d}, J=16.0 \mathrm{~Hz}, 1 \mathrm{H}), 4.28(\mathrm{t}, J=6.7 \mathrm{~Hz}, 2 \mathrm{H})$, 1.70-1.77 (m, 2H), 1.43-1.52 (m, 2H), $1.00(\mathrm{t}, J=7.4 \mathrm{~Hz}, 3 \mathrm{H}) ;{ }^{13} \mathrm{C} \mathrm{NMR}\left(100 \mathrm{MHz}, \mathrm{CDCl}_{3}\right) \delta 167.7,141.2$, 134.3, 128.9, 124.9, 123.3, 122.0, 120.0, 118.2, 117.8, 103.1, 64.6, 30.7, 19.2, 13.7; HRMS $m / z:[\mathrm{M}+\mathrm{H}]^{+}$ Calcd for $\mathrm{C}_{15} \mathrm{H}_{18} \mathrm{NO}_{2}$ 244.1332; Found 244.1336. 
Methyl (E)-3-(1H-indol-7-yl)acrylate $(\mathbf{3 b b})^{2}$

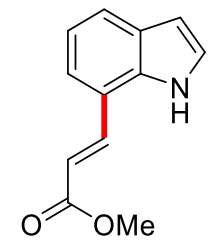

White solid (35 mg, 86\%); $\left(\mathrm{R}_{f}=0.4,20 \%\right.$ Ethyl acetate/Hexane); $\mathrm{mp} 94-96{ }^{\circ} \mathrm{C} ;{ }^{1} \mathrm{H}$ NMR $\left(400 \mathrm{MHz}, \mathrm{CDCl}_{3}\right)$ $\delta 8.73(\mathrm{bs}, 1 \mathrm{H}), 8.10(\mathrm{~d}, J=16.0 \mathrm{~Hz}, 1 \mathrm{H}), 7.70-7.72(\mathrm{~m}, 1 \mathrm{H}), 7.43$ (d, $J=7.5 \mathrm{~Hz}, 1 \mathrm{H}), 7.26-7.28$ $(\mathrm{m}, 1 \mathrm{H}), 7.16(\mathrm{t}, J=7.6 \mathrm{~Hz}, 1 \mathrm{H}), 6.62(\mathrm{dd}, J=3.2,2.0 \mathrm{~Hz}, 1 \mathrm{H}), 6.54(\mathrm{~d}, J=16.0 \mathrm{~Hz}, 1 \mathrm{H}), 3.85(\mathrm{~s}$, $3 \mathrm{H}) ;{ }^{13} \mathrm{C} \mathrm{NMR}\left(100 \mathrm{MHz}, \mathrm{CDCl}_{3}\right) \delta 167.8,141.3,134.3,128.9,124.8,123.4,122.2,120.1,118.1$, 117.5, 103.3, 51.8; HRMS m/z: [M+H] $]^{+}$Calcd for $\mathrm{C}_{12} \mathrm{H}_{12} \mathrm{NO}_{2}$ 202.0863; Found 202.0864.

Ethyl (E)-3-(1H-indol-7-yl)acrylate (3bc $)^{3}$<smiles>CCOC(=O)/C=C/c1cccc2cc[nH]c12</smiles>

White solid (40 mg, 91\%); ( $\mathrm{R}_{f}=0.4,20 \%$ Ethyl acetate/Hexane); mp 98-100 ${ }^{\circ} \mathrm{C} ;{ }^{1} \mathrm{H}$ NMR (400 MHz, $\left.\mathrm{CDCl}_{3}\right) \delta 8.79(\mathrm{bs}, 1 \mathrm{H}), 8.07(\mathrm{~d}, J=16.0 \mathrm{~Hz}, 1 \mathrm{H}), 7.70-7.72(\mathrm{~m}, 1 \mathrm{H}), 7.43(\mathrm{~d}, J=7.4 \mathrm{~Hz}, 1 \mathrm{H}), 7.26-$ $7.27(\mathrm{~m}, 1 \mathrm{H}), 7.16(\mathrm{t}, J=7.6 \mathrm{~Hz}, 1 \mathrm{H}), 6.61(\mathrm{dd}, J=3.2,2.0 \mathrm{~Hz}, 1 \mathrm{H}), 6.54(\mathrm{~d}, J=16.0 \mathrm{~Hz}, 1 \mathrm{H})$, $4.31(\mathrm{q}, J=7.1 \mathrm{~Hz}, 2 \mathrm{H}), 1.37(\mathrm{t}, J=7.1 \mathrm{~Hz}, 3 \mathrm{H}) ;{ }^{13} \mathrm{C} \mathrm{NMR}\left(100 \mathrm{MHz}, \mathrm{CDCl}_{3}\right) \delta 167.4,141.1,134.3$, 128.9, 124.8, 123.3, 122.3, 120.1, 118.2, 118.0, 103.3, 60.6, 14.3; HRMS m/z: $[\mathrm{M}+\mathrm{H}]^{+}$Calcd for $\mathrm{C}_{13} \mathrm{H}_{14} \mathrm{NO}_{2} 216.1019$; Found 216.1024.

iso-Butyl (E)-3-(1H-indol-7-yl)acrylate (3bd)<smiles>CCCCOC(=O)/C=C/c1cccc2cc[nH]c12</smiles>

White solid (41 mg, 83\%); ( $\mathrm{R}_{f}=0.5,5 \%$ Ethyl acetate/Hexane); mp 113-115 ${ }^{\circ} \mathrm{C} ;{ }^{1} \mathrm{H}$ NMR (400 MHz, $\left.\mathrm{CDCl}_{3}\right) \delta 8.80(\mathrm{bs}, 1 \mathrm{H}), 8.08(\mathrm{~d}, J=16.0 \mathrm{~Hz}, 1 \mathrm{H}), 7.70-7.72(\mathrm{~m}, 1 \mathrm{H}), 7.44(\mathrm{~d}, J=7.4 \mathrm{~Hz}, 1 \mathrm{H}), 7.26-7.28$ $(\mathrm{m}, 1 \mathrm{H}), 7.16(\mathrm{t}, J=7.6 \mathrm{~Hz}, 1 \mathrm{H}), 6.62(\mathrm{dd}, J=3.2,2.0 \mathrm{~Hz}, 1 \mathrm{H}), 6.56(\mathrm{~d}, J=16.0 \mathrm{~Hz}, 1 \mathrm{H}), 4.04(\mathrm{~d}, J=6.7$ $\mathrm{Hz}, 2 \mathrm{H}), 2.00-2.10(\mathrm{~m}, 1 \mathrm{H}), 1.00(\mathrm{~d}, J=6.7 \mathrm{~Hz}, 6 \mathrm{H}) ;{ }^{13} \mathrm{C} \mathrm{NMR}\left(100 \mathrm{MHz}, \mathrm{CDCl}_{3}\right) \delta 167.5,141.1,134.3$, 
$128.9,124.8,123.3,122.3,120.1,118.3,117.9,103.3,70.8,27.8,19.2$; HRMS $m / z:[\mathrm{M}+\mathrm{H}]^{+}$Calcd for $\mathrm{C}_{15} \mathrm{H}_{18} \mathrm{NO}_{2} 244.1332 ;$ Found 244.1334.

tert-Butyl (E)-3-(1H-indol-7-yl)acrylate (3be)

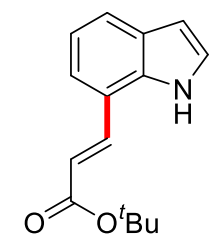

White solid (39 mg, 79\%); ( $\mathrm{R}_{f}=0.4,20 \%$ Ethyl acetate/Hexane); mp 109-111 ${ }^{\circ} \mathrm{C} ;{ }^{1} \mathrm{H}$ NMR (400 MHz, $\left.\mathrm{CDCl}_{3}\right) \delta 8.58(\mathrm{bs}, 1 \mathrm{H}), 7.92(\mathrm{~d}, J=16.0 \mathrm{~Hz}, 1 \mathrm{H}), 7.68-7.70(\mathrm{~m}, 1 \mathrm{H}), 7.39$ (d, $\left.J=7.4 \mathrm{~Hz}, 1 \mathrm{H}\right), 7.26-7.27$ (m, 1H), $7.14(\mathrm{t}, J=7.6 \mathrm{~Hz}, 1 \mathrm{H}), 6.61(\mathrm{dd}, J=3.2,2.0 \mathrm{~Hz}, 1 \mathrm{H}), 6.44(\mathrm{~d}, J=16.0 \mathrm{~Hz}, 1 \mathrm{H}), 1.57(\mathrm{~s}, 9 \mathrm{H}) ;{ }^{13} \mathrm{C}$ $\mathrm{NMR}\left(100 \mathrm{MHz}, \mathrm{CDCl}_{3}\right) \delta 166.6,140.1,134.2,128.8,124.7,123.0,122.5,120.1,120.0,118.4,103.3,80.7$, 28.2; HRMS $m / z:[\mathrm{M}+\mathrm{H}]^{+}$Calcd for $\mathrm{C}_{15} \mathrm{H}_{18} \mathrm{NO}_{2}$ 244.1332; Found 244.1339.

Cyclohexyl (E)-3-(1H-indol-7-yl)acrylate (3bf)<smiles>O=C(/C=C/c1cccc2cc[nH]c12)OCCO</smiles>

White solid (46 mg, 84\%); $\left(\mathrm{R}_{f}=0.4,20 \%\right.$ Ethyl acetate/Hexane); mp 105-107 ${ }^{\circ} \mathrm{C} ;{ }^{1} \mathrm{H}$ NMR (400 MHz, $\left.\mathrm{CDCl}_{3}\right) \delta 8.04(\mathrm{~d}, J=16.0 \mathrm{~Hz}, 1 \mathrm{H}), 7.69-7.71(\mathrm{~m}, 1 \mathrm{H}), 7.43(\mathrm{~d}, J=7.4 \mathrm{~Hz}, 1 \mathrm{H}), 7.26-7.27(\mathrm{~m}, 1 \mathrm{H}), 7.15(\mathrm{t}$, $J=7.6 \mathrm{~Hz}, 1 \mathrm{H}), 6.61(\mathrm{dd}, J=3.2,2.0 \mathrm{~Hz}, 1 \mathrm{H}), 6.53$ (d, $J=16.0 \mathrm{~Hz}, 1 \mathrm{H}), 4.90-4.97$ (m, 1H), 1.94-1.99 (m, $2 \mathrm{H}), 1.76-1.82(\mathrm{~m}, 2 \mathrm{H}), 1.26-1.62(\mathrm{~m}, 6 \mathrm{H}), 8.77$ (bs, $1 \mathrm{H}) ;{ }^{13} \mathrm{C} \mathrm{NMR}\left(100 \mathrm{MHz}, \mathrm{CDCl}_{3}\right) \delta 166.8,140.9$, 134.2, 128.9, 124.8, 123.2, 122.3, 120.1, 118.6, 118.3, 103.2, 72.9, 31.8, 25.4, 23.8; HRMS m/z: [M+H] ${ }^{+}$ Calcd for $\mathrm{C}_{17} \mathrm{H}_{20} \mathrm{NO}_{2} 270.1489$; Found 270.1502.

Phenyl (E)-3-(1H-indol-7-yl)acrylate (3bg)<smiles>O=C(/C=C/c1cccc2cc[nH]c12)c1ccccc1</smiles>

White solid (39 mg, 74\%); $\left(\mathrm{R}_{f}=0.4,20 \%\right.$ Ethyl acetate/Hexane); mp 174-176 ${ }^{\circ} \mathrm{C} ;{ }^{1} \mathrm{H}$ NMR (400 MHz, $\left.\mathrm{CDCl}_{3}\right) \delta 8.84(\mathrm{bs}, 1 \mathrm{H}), 8.28(\mathrm{~d}, J=16.0 \mathrm{~Hz}, 1 \mathrm{H}), 7.73-7.75(\mathrm{~m}, 1 \mathrm{H}), 7.50(\mathrm{~d}, J=7.5 \mathrm{~Hz}, 1 \mathrm{H}), 7.41-7.45$ $(\mathrm{m}, 2 \mathrm{H}), 7.26-7.30(\mathrm{~m}, 1 \mathrm{H}), 7.16-7.23(\mathrm{~m}, 3 \mathrm{H}), 7.08(\mathrm{t}, J=2.9 \mathrm{~Hz}, 1 \mathrm{H}), 6.74(\mathrm{~d}, J=16.0 \mathrm{~Hz}, 1 \mathrm{H}), 6.60(\mathrm{dd}$, 
$J=3.2,2.0 \mathrm{~Hz}, 1 \mathrm{H}) ;{ }^{13} \mathrm{C} \mathrm{NMR}\left(100 \mathrm{MHz}, \mathrm{CDCl}_{3}\right) \delta 165.9,150.8,143.2,134.4,129.5,129.0,125.9,125.0$, 123.9, 122.5, 121.7, 120.1, 118.0, 116.8, 103.2; HRMS m/z: $[\mathrm{M}+\mathrm{H}]^{+}$Calcd for $\mathrm{C}_{17} \mathrm{H}_{14} \mathrm{NO}_{2}$ 264.1019; Found 264.0999 .

(E)-4-(1H-indol-7-yl)but-3-en-2-one (3bh)

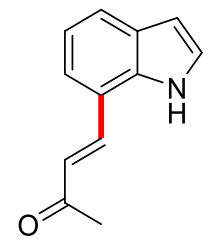

Pale green solid (21 mg, 56\%); $\left(\mathrm{R}_{f}=0.4,20 \%\right.$ Ethyl acetate/Hexane); mp 126-128 ${ }^{\circ} \mathrm{C} ;{ }^{1} \mathrm{H}$ NMR (400 MHz, $\left.\mathrm{CDCl}_{3}\right) \delta 9.10(\mathrm{bs}, 1 \mathrm{H}), 7.97(\mathrm{~d}, J=16.2 \mathrm{~Hz}, 1 \mathrm{H}), 7.73(\mathrm{dt}, J=8.0,1.0 \mathrm{~Hz}, 1 \mathrm{H}), 7.44(\mathrm{~d}, J=7.5 \mathrm{~Hz}, 1 \mathrm{H})$, $7.29(\mathrm{t}, J=2.8 \mathrm{~Hz}, 1 \mathrm{H}), 7.16(\mathrm{t}, J=7.6 \mathrm{~Hz}, 1 \mathrm{H}), 6.87(\mathrm{~d}, J=16.2 \mathrm{~Hz}, 1 \mathrm{H}), 6.62(\mathrm{dd}, J=3.2,2.0 \mathrm{~Hz}, 1 \mathrm{H})$, 2.44 (s, 3H); ${ }^{13} \mathrm{C}$ NMR $\left(100 \mathrm{MHz}, \mathrm{CDCl}_{3}\right) \delta 198.5,140.3,134.5,129.1,126.4,124.9,123.8,122.7,120.1$, 118.2, 103.3, 28.2; HRMS m/z: [M+H] ${ }^{+}$Calcd for $\mathrm{C}_{12} \mathrm{H}_{12} \mathrm{NO}$ 186.0913; Found 186.0909.

(E)-3-(1H-indol-7-yl)acrylonitrile (3bi)

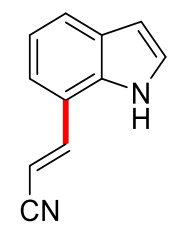

White solid (8 mg, 22\%); ( $\mathrm{R}_{f}=0.4,20 \%$ Ethyl acetate/Hexane); mp 145-147 ${ }^{\circ} \mathrm{C} ;{ }^{1} \mathrm{H}$ NMR (400 MHz, $\left.\mathrm{CDCl}_{3}\right) \delta 8.78(\mathrm{bs}, 1 \mathrm{H}), 7.88(\mathrm{~d}, J=16.5 \mathrm{~Hz}, 1 \mathrm{H}), 7.75(\mathrm{~d}, J=7.8 \mathrm{~Hz}, 1 \mathrm{H}), 7.37(\mathrm{~d}, J=7.4 \mathrm{~Hz}, 1 \mathrm{H}), 7.30$ $(\mathrm{t}, J=2.9 \mathrm{~Hz}, 1 \mathrm{H}), 7.16(\mathrm{t}, J=7.6 \mathrm{~Hz}, 1 \mathrm{H}), 6.62(\mathrm{dd}, J=3.3,2.0 \mathrm{~Hz}, 1 \mathrm{H}), 5.97(\mathrm{~d}, J=16.5 \mathrm{~Hz}, 1 \mathrm{H}) ;{ }^{13} \mathrm{C}$ NMR (100 MHz, $\left.\mathrm{CDCl}_{3}\right) \delta 147.3,134.0,129.2,125.3,124.4,120.7,120.1,118.8,117.5,103.3$, 95.4; HRMS $m / z:[\mathrm{M}+\mathrm{H}]^{+}$Calcd for $\mathrm{C}_{11} \mathrm{H}_{9} \mathrm{~N}_{2}$ 169.0760; Found 169.0769.

(E)-7-Styryl-1H-indole $(\mathbf{3 b j})^{3}$

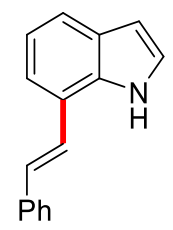

White solid (5 mg, 12\%); $\left(\mathrm{R}_{f}=0.3,10 \%\right.$ Ethyl acetate/Hexane); mp 95-97 ${ }^{\circ} \mathrm{C} ;{ }^{1} \mathrm{H} \mathrm{NMR}\left(400 \mathrm{MHz}, \mathrm{CDCl}_{3}\right)$ $\delta 8.49(\mathrm{bs}, 1 \mathrm{H}), 7.55-7.61(\mathrm{~m}, 3 \mathrm{H}), 7.37-7.42(\mathrm{~m}, 4 \mathrm{H}), 7.27-7.31(\mathrm{~m}, 2 \mathrm{H}), 7.14-7.21(\mathrm{~m}, 2 \mathrm{H}), 6.61(\mathrm{dd}, J=$ 3.2, $2.0 \mathrm{~Hz}, 1 \mathrm{H}) ;{ }^{13} \mathrm{C} \mathrm{NMR}\left(100 \mathrm{MHz}, \mathrm{CDCl}_{3}\right) \delta 137.5,133.7,129.8,128.8,128.5,127.7,126.4,125.1$, 
124.2, 121.4, 120.50, 120.47, 120.2, 103.3; HRMS m/z: $[\mathrm{M}+\mathrm{H}]^{+}$Calcd for $\mathrm{C}_{16} \mathrm{H}_{14} \mathrm{~N}$ 220.1121; Found 220.1137 .

Butyl (E)-3-(5-methyl-1H-indol-7-yl)acrylate (3da)

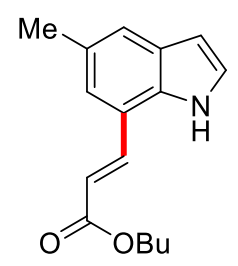

White solid (40 mg, 77\%); $\left(\mathrm{R}_{f}=0.4,20 \%\right.$ Ethyl acetate/Hexane); $\mathrm{mp} 94-96{ }^{\circ} \mathrm{C} ;{ }^{1} \mathrm{H} \mathrm{NMR}\left(400 \mathrm{MHz}, \mathrm{CDCl}_{3}\right)$ $\delta 8.72(\mathrm{bs}, 1 \mathrm{H}), 8.03(\mathrm{~d}, J=16.0 \mathrm{~Hz}, 1 \mathrm{H}), 7.50(\mathrm{~s}, 1 \mathrm{H}), 7.26-7.27(\mathrm{~m}, 1 \mathrm{H}), 7.22(\mathrm{t}, J=2.9 \mathrm{~Hz}, 1 \mathrm{H}), 6.51-$ $6.55(\mathrm{~m}, 2 \mathrm{H}), 4.26(\mathrm{t}, J=6.7 \mathrm{~Hz}, 2 \mathrm{H}), 2.46(\mathrm{~s}, 3 \mathrm{H}), 1.69-1.76(\mathrm{~m}, 2 \mathrm{H}), 1.42-1.51(\mathrm{~m}, 2 \mathrm{H}), 0.99(\mathrm{t}, J=7.4$ $\mathrm{Hz}, 3 \mathrm{H}) ;{ }^{13} \mathrm{C} \mathrm{NMR}\left(100 \mathrm{MHz}, \mathrm{CDCl}_{3}\right) \delta 167.5,141.3,132.8,129.3,124.9,123.7,123.3,117.9,117.7,102.7$, 64.5, 30.8, 21.8, 19.2, 13.7 (1C overlapped); HRMS $m / z$ : $[\mathrm{M}+\mathrm{H}]^{+}$Calcd for $\mathrm{C}_{16} \mathrm{H}_{20} \mathrm{NO}_{2}$ 258.1489; Found 258.1499 .

Butyl (E)-3-(5-methoxy-1H-indol-7-yl)acrylate (3ea)

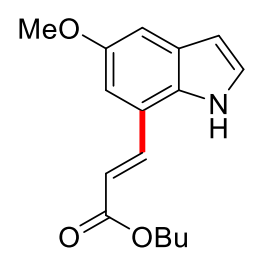

White solid (43 mg, 78\%); $\left(\mathrm{R}_{f}=0.4,20 \%\right.$ Ethyl acetate/Hexane); mp 76-78 ${ }^{\circ} \mathrm{C} ;{ }^{1} \mathrm{H} \mathrm{NMR}\left(400 \mathrm{MHz}, \mathrm{CDCl}_{3}\right)$ $\delta 8.57(\mathrm{bs}, 1 \mathrm{H}), 7.98(\mathrm{~d}, J=16.0,1 \mathrm{H}), 7.24(\mathrm{t}, J=2.8 \mathrm{~Hz}, 1 \mathrm{H}), 7.20(\mathrm{~d}, J=2.4 \mathrm{~Hz}, 1 \mathrm{H}), 7.07(\mathrm{~d}, J=2.4$ $\mathrm{Hz}, 1 \mathrm{H}), 6.49-6.54(\mathrm{~m}, 2 \mathrm{H}), 4.25$ (t, $J=6.7 \mathrm{~Hz}, 2 \mathrm{H}), 3.87$ (s, 3H), 1.68-1.73 (m, 2H), 1.41-1.50 (m, 2H), $0.98(\mathrm{t}, J=7.4 \mathrm{~Hz}, 3 \mathrm{H}){ }^{13} \mathrm{C} \mathrm{NMR}\left(100 \mathrm{MHz}, \mathrm{CDCl}_{3}\right) \delta 167.3,154.2,140.6,129.8,129.5,125.5,118.7$, 118.6, 111.1, 105.6, 102.9, 64.6, 56.0, 30.8, 19.2, 13.7; HRMS m/z: [M+H] $]^{+}$Calcd for $\mathrm{C}_{16} \mathrm{H}_{20} \mathrm{NO}_{3} 274.1438$; Found 274.1424.

Butyl (E)-3-(5-chloro-1 $H$-indol-7-yl)acrylate (3fa)

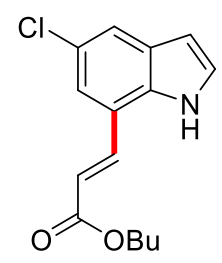

White solid (46 mg, 82\%); ( $\mathrm{R}_{f}=0.4,20 \%$ Ethyl acetate/Hexane); $\mathrm{mp} 83-85{ }^{\circ} \mathrm{C} ;{ }^{1} \mathrm{H} \mathrm{NMR}\left(400 \mathrm{MHz}, \mathrm{CDCl}_{3}\right)$ $\delta 8.94(\mathrm{bs}, 1 \mathrm{H}), 8.00(\mathrm{~d}, J=16.0 \mathrm{~Hz}, 1 \mathrm{H}), 7.64(\mathrm{~d}, J=1.7 \mathrm{~Hz}, 1 \mathrm{H}), 7.39(\mathrm{~d}, J=1.9 \mathrm{~Hz}, 1 \mathrm{H}), 7.28(\mathrm{t}, J=2.9$ 
$\mathrm{Hz}, 1 \mathrm{H}), 6.53-6.57(\mathrm{~m}, 2 \mathrm{H}), 4.25(\mathrm{t}, J=6.7 \mathrm{~Hz}, 2 \mathrm{H}), 1.68-1.75(\mathrm{~m}, 2 \mathrm{H}), 1.40-1.50(\mathrm{~m}, 2 \mathrm{H}), 0.97(\mathrm{t}, J=7.4$ $\mathrm{Hz}, 3 \mathrm{H}) ;{ }^{13} \mathrm{C} \mathrm{NMR}\left(100 \mathrm{MHz}, \mathrm{CDCl}_{3}\right) \delta 167.2,139.5,132.9,129.9,126.1,125.7,122.3,121.4,119.4$, 119.2, 102.9, 64.8, 30.7, 19.2, 13.7; HRMS m/z: $[\mathrm{M}+\mathrm{H}]^{+}$Calcd for $\mathrm{C}_{15} \mathrm{H}_{17} \mathrm{ClNO}_{2}$ 278.0942; Found 278.0927.

Butyl (E)-3-(5-bromo-1H-indol-7-yl)acrylate (3ga)

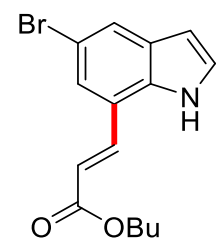

White solid (55 mg, 85\%); $\left(\mathrm{R}_{f}=0.4,20 \%\right.$ Ethyl acetate/Hexane); $\mathrm{mp} 97-99{ }^{\circ} \mathrm{C} ;{ }^{1} \mathrm{H} \mathrm{NMR}\left(400 \mathrm{MHz}, \mathrm{CDCl}_{3}\right)$ $\delta 8.84(\mathrm{bs}, 1 \mathrm{H}), 7.96(\mathrm{~d}, J=16.0 \mathrm{~Hz}, 1 \mathrm{H}), 7.79(\mathrm{~d}, J=1.8 \mathrm{~Hz}, 1 \mathrm{H}), 7.52(\mathrm{~d}, J=1.8 \mathrm{~Hz}, 1 \mathrm{H}), 7.27(\mathrm{t}, J=2.9$ $\mathrm{Hz}, 1 \mathrm{H}), 6.51-6.56(\mathrm{~m}, 2 \mathrm{H}), 4.25(\mathrm{t}, J=6.7 \mathrm{~Hz}, 2 \mathrm{H}), 1.67-1.75(\mathrm{~m}, 2 \mathrm{H}), 1.40-1.50(\mathrm{~m}, 2 \mathrm{H}), 0.97(\mathrm{t}, J=7.4$ $\mathrm{Hz}, 3 \mathrm{H}) ;{ }^{13} \mathrm{C} \mathrm{NMR}\left(100 \mathrm{MHz}, \mathrm{CDCl}_{3}\right) \delta 167.1,139.3,133.1,130.5,126.0,125.3,124.1,119.7,119.5$, 113.2, 102.9, 64.8, 30.7, 19.2, 13.7; HRMS $m / z:[\mathrm{M}+\mathrm{H}]^{+}$Calcd for $\mathrm{C}_{15} \mathrm{H}_{17} \mathrm{BrNO}_{2}$ 322.0437; Found 322.0425 .

Butyl (E)-3-(6-chloro-1H-indol-7-yl)acrylate (3ha)<smiles>CCCCC(=O)/C=C/c1c(Cl)ccc2cc[nH]c12</smiles>

White solid (16 mg, 29\%); $\left(\mathrm{R}_{f}=0.4,20 \%\right.$ Ethyl acetate/Hexane); $\mathrm{mp} 80-82{ }^{\circ} \mathrm{C} ;{ }^{1} \mathrm{H} \mathrm{NMR}\left(400 \mathrm{MHz}, \mathrm{CDCl}_{3}\right)$ $\delta 8.69(\mathrm{bs}, 1 \mathrm{H}), 8.23(\mathrm{~d}, J=16.4 \mathrm{~Hz}, 1 \mathrm{H}), 7.57(\mathrm{~d}, J=8.4 \mathrm{~Hz}, 1 \mathrm{H}), 7.25-7.27(\mathrm{~m}, 1 \mathrm{H}), 7.19(\mathrm{~d}, J=8.4 \mathrm{~Hz}$, $1 \mathrm{H}), 6.54-6.59(\mathrm{~m}, 2 \mathrm{H}), 4.27(\mathrm{t}, J=6.7 \mathrm{~Hz}, 2 \mathrm{H}), 1.70-1.77(\mathrm{~m}, 2 \mathrm{H}), 1.42-1.52(\mathrm{~m}, 2 \mathrm{H}), 0.99$ (t, $J=7.4 \mathrm{~Hz}$, $3 \mathrm{H}) ;{ }^{13} \mathrm{C} \mathrm{NMR}\left(100 \mathrm{MHz}, \mathrm{CDCl}_{3}\right) \delta 166.7,139.9,134.2,129.2,127.4,125.3,123.2,121.8,121.6,116.9$, 103.6, 64.8, 30.7, 19.2, 13.7,; HRMS $m / z:[\mathrm{M}+\mathrm{H}]^{+}$Calcd for $\mathrm{C}_{15} \mathrm{H}_{17} \mathrm{ClNO}_{2} 278.0942$; Found 278.0949.

Butyl (E)-3-(6-bromo-1H-indol-7-yl)acrylate (3ia)<smiles>CCCCC(=O)/C=C/c1c(Br)ccc2cc[nH]c12</smiles> 
White solid (17 mg, 27\%); $\left(\mathrm{R}_{f}=0.4,20 \%\right.$ Ethyl acetate/Hexane); mp 92-94 ${ }^{\circ} \mathrm{C} ;{ }^{1} \mathrm{H}$ NMR $\left(400 \mathrm{MHz}, \mathrm{CDCl}_{3}\right)$ $\delta 8.63(\mathrm{bs}, 1 \mathrm{H}), 8.16(\mathrm{~d}, J=16.4 \mathrm{~Hz}, 1 \mathrm{H}), 7.49(\mathrm{~d}, J=8.4 \mathrm{~Hz}, 1 \mathrm{H}), 7.35(\mathrm{~d}, J=8.4 \mathrm{~Hz}, 1 \mathrm{H}), 7.24(\mathrm{t}, J=2.9$ $\mathrm{Hz}, 1 \mathrm{H}), 6.57(\mathrm{dd}, J=3.2,2.0 \mathrm{~Hz}, 1 \mathrm{H}), 6.50(\mathrm{~d}, J=16.4 \mathrm{~Hz}, 1 \mathrm{H}), 4.27$ (t, $J=6.7 \mathrm{~Hz}, 2 \mathrm{H}), 1.69-1.76$ (m, $2 \mathrm{H}), 1.42-1.51(\mathrm{~m}, 2 \mathrm{H}), 0.98(\mathrm{t}, J=7.4 \mathrm{~Hz}, 3 \mathrm{H}) ;{ }^{13} \mathrm{C} \mathrm{NMR}\left(100 \mathrm{MHz}, \mathrm{CDCl}_{3}\right) \delta 166.6,142.4,134.3,127.9$, 125.2, 124.7, 123.4, 121.8, 119.0, 118.7, 103.7, 64.8, 30.7, 19.2, 13.8; HRMS $m / z:[\mathrm{M}+\mathrm{H}]^{+}$Calcd for $\mathrm{C}_{15} \mathrm{H}_{17} \mathrm{BrNO}_{2}$ 322.0437; Found 322.0426, 324.0404.

Butyl (E)-3-(3-methyl-1H-indol-7-yl)acrylate (3ka)

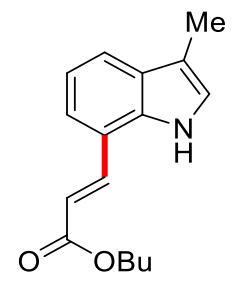

Single crystals suitable for the X-ray analysis were obtained by slow evaporation from the $n$-hexane solution. White solid (45 mg, 86\%); ( $\mathrm{R}_{f}=0.4,20 \%$ Ethyl acetate/Hexane); $\mathrm{mp} 114-116{ }^{\circ} \mathrm{C} ;{ }^{1} \mathrm{H}$ NMR (400 MHz, $\left.\mathrm{CDCl}_{3}\right) \delta 8.37(\mathrm{bs}, 1 \mathrm{H}), 8.01(\mathrm{~d}, J=16.0,1 \mathrm{H}), 7.63(\mathrm{~d}, J=7.8 \mathrm{~Hz}, 1 \mathrm{H}), 7.41(\mathrm{~d}, J=7.4 \mathrm{~Hz}, 1 \mathrm{H}), 7.15(\mathrm{t}, J$ $=7.6 \mathrm{~Hz}, 1 \mathrm{H}), 7.02-7.03(\mathrm{~m}, 1 \mathrm{H}), 6.50(\mathrm{~d}, J=16.0 \mathrm{~Hz}, 1 \mathrm{H}), 4.25(\mathrm{t}, J=6.7 \mathrm{~Hz}, 2 \mathrm{H}), 2.34(\mathrm{~d}, J=1.1 \mathrm{~Hz}$, $3 \mathrm{H}), 1.68-1.75(\mathrm{~m}, 2 \mathrm{H}), 1.41-1.51(\mathrm{~m}, 2 \mathrm{H}), 0.98(\mathrm{t}, J=7.4 \mathrm{~Hz}, 3 \mathrm{H}) ;{ }^{13} \mathrm{C}$ NMR $\left(100 \mathrm{MHz}, \mathrm{CDCl}_{3}\right) \delta 167.4$, 141.2, 134.7, 129.4, 122.4, 122.2, 121.4, 119.4, 118.1, 117.8, 112.4, 64.5, 30.8, 19.2, 13.8, 9.6; HRMS m/z: $[\mathrm{M}+\mathrm{H}]^{+}$Calcd for $\mathrm{C}_{16} \mathrm{H}_{20} \mathrm{NO}_{2}$ 258.1489; Found 258.1473.

Butyl (E)-3-(2-methyl-1H-indol-7-yl)acrylate (3la)

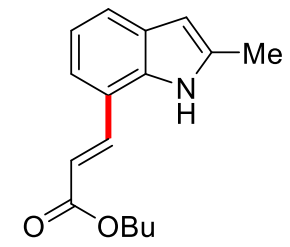

Pale red gum (40 mg, 76\%); $\left(\mathrm{R}_{f}=0.4,20 \%\right.$ Ethyl acetate/Hexane); ${ }^{1} \mathrm{H}$ NMR (400 MHz, $\left.\mathrm{CDCl}_{3}\right) 8.29$ (bs, 1H), $7.99(\mathrm{~d}, J=16.0 \mathrm{~Hz}, 1 \mathrm{H}), 7.55(\mathrm{~d}, J=7.8 \mathrm{~Hz}, 1 \mathrm{H}), 7.32$ (d, $J=7.4 \mathrm{~Hz}, 1 \mathrm{H}), 7.09$ (t, $J=7.6 \mathrm{~Hz}, 1 \mathrm{H})$, $6.48(\mathrm{~d}, J=16.0 \mathrm{~Hz}, 1 \mathrm{H}), 6.26-6.27(\mathrm{~m}, 1 \mathrm{H}), 4.24(\mathrm{t}, J=6.7 \mathrm{~Hz}, 2 \mathrm{H}), 2.49(\mathrm{~d}, J=0.9 \mathrm{~Hz}, 3 \mathrm{H}), 1.68-1.76$ $(\mathrm{m}, 2 \mathrm{H}), 1.41-1.51(\mathrm{~m}, 2 \mathrm{H}), \delta 0.98(\mathrm{t}, J=7.4 \mathrm{~Hz}, 3 \mathrm{H}) ;{ }^{13} \mathrm{C} \mathrm{NMR}\left(100 \mathrm{MHz}, \mathrm{CDCl}_{3}\right) \delta 13.8,19.2,30.8$, 64.5, 101.1, 117.4, 117.7, 120.0, 121.3, 122.2, 130.1, 134.6, 135.8, 141.3, 167.5 (1C overlapped); HRMS $m / z:[\mathrm{M}+\mathrm{H}]^{+}$Calcd for $\mathrm{C}_{16} \mathrm{H}_{20} \mathrm{NO}_{2}$ 258.1489; Found 258.1493.

Butyl (E)-3-(2-phenyl-1 $H$-indol-7-yl)acrylate (3ma) 


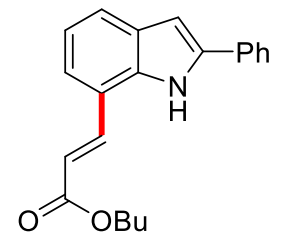

Orange gum (35 mg, 55\%); $\left(\mathrm{R}_{f}=0.4,20 \%\right.$ Ethyl acetate/Hexane); ${ }^{1} \mathrm{H}$ NMR $\left(400 \mathrm{MHz}, \mathrm{CDCl}_{3}\right) \delta 8.93$ (bs, $1 \mathrm{H}), 8.16(\mathrm{~d}, J=16.0 \mathrm{~Hz}, 1 \mathrm{H}), 7.71-7.73(\mathrm{~m}, 2 \mathrm{H}), 7.67-7.69(\mathrm{~m}, 1 \mathrm{H}), 7.43-7.49(\mathrm{~m}, 3 \mathrm{H}), 7.34-7.38(\mathrm{~m}$, $1 \mathrm{H}), 7.15(\mathrm{t}, J=7.6 \mathrm{~Hz}, 1 \mathrm{H}), 6.86(\mathrm{~d}, J=2.0 \mathrm{~Hz}, 1 \mathrm{H}), 6.58(\mathrm{~d}, J=15.9 \mathrm{~Hz}, 1 \mathrm{H}), 4.20(\mathrm{t}, J=6.7 \mathrm{~Hz}, 2 \mathrm{H})$, $1.65-1.72(\mathrm{~m}, 2 \mathrm{H}), 1.38-1.47(\mathrm{~m}, 2 \mathrm{H}), 0.96(\mathrm{t}, J=7.4 \mathrm{~Hz}, 3 \mathrm{H}) ;{ }^{13} \mathrm{C} \mathrm{NMR}\left(100 \mathrm{MHz}, \mathrm{CDCl}_{3}\right) \delta 167.6,140.7$, 138.8, 135.6, 131.9, 130.3, 129.0, 128.0, 125.5, 123.0, 121.7, 120.5, 118.1, 118.0, 100.4, 64.6, 30.7, 19.2, 13.7; HRMS $m / z$ : $[\mathrm{M}+\mathrm{H}]^{+}$Calcd for $\mathrm{C}_{21} \mathrm{H}_{22} \mathrm{NO}_{2}$ 320.1645; Found 320.1638 .

Butyl (E)-3-(2-(naphthalen-2-yl)-1H-indol-7-yl)acrylate (3na)

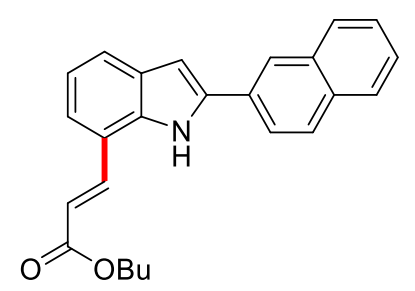

White solid (37 mg, 50\%); $\left(\mathrm{R}_{f}=0.4,20 \%\right.$ Ethyl acetate/Hexane); mp 107-109 ${ }^{\circ} \mathrm{C} ;{ }^{1} \mathrm{H}$ NMR (400 MHz, $\left.\mathrm{CDCl}_{3}\right) \delta 8.92(\mathrm{bs}, 1 \mathrm{H}), 8.17(\mathrm{~d}, J=15.9 \mathrm{~Hz}, 1 \mathrm{H}), 8.13-8.14(\mathrm{~m}, 1 \mathrm{H}), 7.92-7.94(\mathrm{~m}, 2 \mathrm{H}), 7.84-7.87(\mathrm{~m}, 2 \mathrm{H})$, $7.70(\mathrm{~d}, J=7.8 \mathrm{~Hz}, 1 \mathrm{H}), 7.45-7.57(\mathrm{~m}, 3 \mathrm{H}), 7.17(\mathrm{t}, J=7.7 \mathrm{~Hz}, 1 \mathrm{H}), 6.99(\mathrm{~d}, J=2.0 \mathrm{~Hz}, 1 \mathrm{H}), 6.61(\mathrm{~d}, J=$ $15.9 \mathrm{~Hz}, 1 \mathrm{H}), 4.25(\mathrm{t}, J=6.7 \mathrm{~Hz}, 2 \mathrm{H}), 1.67-1.74(\mathrm{~m}, 2 \mathrm{H}), 1.40-1.49(\mathrm{~m}, 2 \mathrm{H}), 0.97(\mathrm{t}, J=7.4 \mathrm{~Hz}, 3 \mathrm{H}) ;{ }^{13} \mathrm{C}$ NMR $\left(100 \mathrm{MHz}, \mathrm{CDCl}_{3}\right) \delta 167.6,140.5,138.7,135.8,133.5,133.0,130.3,129.2,128.9,128.1,127.8$, 126.8, 126.3 123.8, 123.5, 123.1, 121.8, 120.6, 118.1, 101.1, 64.6, 30.8, 19.2, 13.7 (1C overlapped); HRMS $m / z:[\mathrm{M}+\mathrm{H}]^{+}$Calcd for $\mathrm{C}_{25} \mathrm{H}_{24} \mathrm{NO}_{2} 370.1802$; Found 370.1800.

Butyl (E)-3-(1,2,3,4-tetrahydrocyclopenta[b]indol-5-yl)acrylate (3oa)

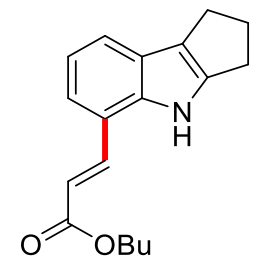

Pale yellow solid (50 mg, 87\%); ( $\mathrm{R}_{f}=0.4,20 \%$ Ethyl acetate/Hexane); mp 89-91 ${ }^{\circ} \mathrm{C} ;{ }^{1} \mathrm{H} \mathrm{NMR}(400 \mathrm{MHz}$, $\left.\mathrm{CDCl}_{3}\right) \delta 8.36(\mathrm{bs}, 1 \mathrm{H}), 8.00(\mathrm{~d}, J=16.0 \mathrm{~Hz}, 1 \mathrm{H}), 7.48(\mathrm{~d}, J=7.8 \mathrm{~Hz}, 1 \mathrm{H}), 7.32(\mathrm{~d}, J=7.4 \mathrm{~Hz}, 1 \mathrm{H}), 7.10$ $(\mathrm{t}, J=7.7 \mathrm{~Hz}, 1 \mathrm{H}), 6.50(\mathrm{~d}, J=16.0 \mathrm{~Hz}, 1 \mathrm{H}), 4.25(\mathrm{t}, J=6.7 \mathrm{~Hz}, 2 \mathrm{H}), 2.82-2.91(\mathrm{~m}, 4 \mathrm{H}), 2.54-2.59(\mathrm{~m}$, 
2H), 1.68-1.75 (m, 2H), 1.41-1.51 (m, 2H), $0.98(\mathrm{t}, J=7.4 \mathrm{~Hz}, 3 \mathrm{H}) ;{ }^{13} \mathrm{C}$ NMR $\left(100 \mathrm{MHz}, \mathrm{CDCl}_{3}\right) \delta 167.5$, 144.5, 141.3, 139.4, 125.7, 121.0, 120.7, 120.4, 119.7, 118.3, 117.7, 64.5, 30.8, 28.6, 25.9, 24.4, 19.2 , 13.7; HRMS m/z: [M+H] ${ }^{+}$Calcd for $\mathrm{C}_{18} \mathrm{H}_{22} \mathrm{NO}_{2} 284.1645$; Found 284.1632.

Butyl (E)-3-(2,3,4,9-tetrahydro-1H-carbazol-8-yl)acrylate (3pa)

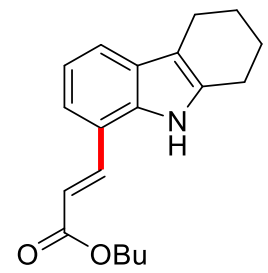

White solid (53 mg, 89\%); ( $\mathrm{R}_{f}=0.4,20 \%$ Ethyl acetate/Hexane); mp 120-122 ${ }^{\circ} \mathrm{C} ;{ }^{1} \mathrm{H}$ NMR (400 MHz, $\left.\mathrm{CDCl}_{3}\right) \delta 8.08(\mathrm{bs}, 1 \mathrm{H}), 7.98(\mathrm{~d}, J=16.0 \mathrm{~Hz}, 1 \mathrm{H}), 7.50(\mathrm{~d}, J=7.8 \mathrm{~Hz}, 1 \mathrm{H}), 7.33(\mathrm{~d}, J=7.4 \mathrm{~Hz}, 1 \mathrm{H}), 7.10$ (t, $J=7.7 \mathrm{~Hz}, 1 \mathrm{H}), 6.48(\mathrm{~d}, J=16.0 \mathrm{~Hz}, 1 \mathrm{H}), 4.24(\mathrm{t}, J=6.7 \mathrm{~Hz}, 2 \mathrm{H}), 2.75-2.78(\mathrm{~m}, 2 \mathrm{H}), 2.69-2.72(\mathrm{~m}$, $2 \mathrm{H}), 1.85-1.97(\mathrm{~m}, 4 \mathrm{H}), 1.68-1.75(\mathrm{~m}, 2 \mathrm{H}), 1.41-1.51(\mathrm{~m}, 2 \mathrm{H}), 0.98(\mathrm{t}, J=7.4 \mathrm{~Hz}, 3 \mathrm{H}) ;{ }^{13} \mathrm{C}$ NMR $(100$ $\left.\mathrm{MHz}, \mathrm{CDCl}_{3}\right) \delta 167.5,141.5,134.9,134.1,128.9,121.6,120.3,119.4,117.5,117.4,110.9,64.5,30.8,23.2$, 23.1, 23.09, 20.8, 19.2, 13.8; HRMS $m / z:[\mathrm{M}+\mathrm{H}]^{+}$Calcd for $\mathrm{C}_{19} \mathrm{H}_{24} \mathrm{NO}_{2}$ 298.1802; Found 298.1803.

Butyl (E)-3-(9H-carbazol-1-yl)acrylate (3qa)<smiles>CCCCOC(=O)/C=C/c1cccc2c1[nH]c1ccccc12</smiles>

White solid (40 mg, 67\%); $\left(\mathrm{R}_{f}=0.4,20 \%\right.$ Ethyl acetate/Hexane); $\mathrm{mp} 90-92{ }^{\circ} \mathrm{C} ;{ }^{1} \mathrm{H} \mathrm{NMR}\left(400 \mathrm{MHz}, \mathrm{CDCl}_{3}\right)$ $\delta 8.68(\mathrm{bs}, 1 \mathrm{H}), 8.05-8.13(\mathrm{~m}, 3 \mathrm{H}), 7.59-7.61(\mathrm{~m}, 1 \mathrm{H}), 7.42-7.48(\mathrm{~m}, 2 \mathrm{H}), 7.22-7.28(\mathrm{~m}, 2 \mathrm{H}), 6.60(\mathrm{~d}, J=$ $16.0 \mathrm{~Hz}, 1 \mathrm{H}), 4.26(\mathrm{t}, J=6.7 \mathrm{~Hz}, 2 \mathrm{H}), 1.68-1.78(\mathrm{~m}, 2 \mathrm{H}), 1.40-1.49(\mathrm{~m}, 2 \mathrm{H}), 0.97(\mathrm{t}, J=7.4 \mathrm{~Hz}, 3 \mathrm{H}) ;{ }^{13} \mathrm{C}$ NMR (100 MHz, $\left.\mathrm{CDCl}_{3}\right) \delta 167.5,140.6,139.6,138.2,126.4,125.2,124.5,123.0,122.4,120.4,120.1$, 119.7, 118.2, 117.6, 111.0, 64.7, 30.8, 19.2, 13.7; HRMS m/z: $[\mathrm{M}+\mathrm{H}]^{+}$Calcd for $\mathrm{C}_{19} \mathrm{H}_{20} \mathrm{NO}_{2}$ 294.1489; Found 294.1492.

Methyl (E)-5-(3-butoxy-3-oxoprop-1-en-1-yl)-1-(cyclohexylthio)-1H-indole-6-carboxylate (4ja)<smiles>CCOC(=O)/C=C/c1cc2ccn(C3CCCCC3)c2cc1C(C)=O</smiles>

This compound was obtained without the TBAF treatment (directing group removal). 
Pale yellow gum (35 mg, 42\%); $\left(\mathrm{R}_{f}=0.3,20 \%\right.$ Ethyl acetate/Hexane); ${ }^{1} \mathrm{H}$ NMR (400 MHz, $\left.\mathrm{CDCl}_{3}\right) \delta 8.55$ $(\mathrm{d}, J=15.7 \mathrm{~Hz}, 1 \mathrm{H}), 8.26(\mathrm{~s}, 1 \mathrm{H}), 7.82(\mathrm{~s}, 1 \mathrm{H}), 7.27(\mathrm{~d}, J=3.3 \mathrm{~Hz}, 1 \mathrm{H}), 6.58(\mathrm{dd}, J=3.3,0.9 \mathrm{~Hz}, 1 \mathrm{H}), 6.30$ $(\mathrm{d}, J=15.7 \mathrm{~Hz}, 1 \mathrm{H}), 4.22(\mathrm{t}, J=6.7 \mathrm{~Hz}, 2 \mathrm{H}), 3.97(\mathrm{~s}, 3 \mathrm{H}), 3.08-3.15(\mathrm{~m}, 1 \mathrm{H}), 1.80-1.86(\mathrm{~m}, 2 \mathrm{H}), 1.67-1.78$ $(\mathrm{m}, 4 \mathrm{H}), 1.58-1.62(\mathrm{~m}, 1 \mathrm{H}), 1.40-1.50(\mathrm{~m}, 2 \mathrm{H}), 1.12-1.34(\mathrm{~m}, 5 \mathrm{H}), 0.97(\mathrm{t}, J=7.4 \mathrm{~Hz}, 3 \mathrm{H}) ;{ }^{13} \mathrm{C}$ NMR $(100$ $\left.\mathrm{MHz}, \mathrm{CDCl}_{3}\right) \delta 168.0,167.1,145.4,140.9,139.5,132.1,129.2,124.4,120.6,118.7,114.7,104.8,64.2$, 52.3, 51.1, 31.3, 30.8, 25.6, 25.4, 19.2, 13.8; HRMS m/z: $[\mathrm{M}+\mathrm{H}]^{+}$Calcd for $\mathrm{C}_{23} \mathrm{H}_{30} \mathrm{NO}_{4} \mathrm{~S} 416.1890$; Found 416.1877. 


\section{4-4. $\mathrm{AuCl}_{3}$-catalyzed Michael addition to acrolein (Scheme 5)}

To a round-bottom flask equipped with a magnetic stirrer bar were added $\mathbf{3 b a}$ ( $25 \mathrm{mg}, 0.1 \mathrm{mmol}$ ), acrolein (10 $\mu \mathrm{L}, 0.15 \mathrm{mmol})$, and $\mathrm{CH}_{3} \mathrm{CN}(5.0 \mathrm{~mL}) . \mathrm{AuCl}_{3}(1.5 \mathrm{mg}, 0.005 \mathrm{mmol})$ was added to the flask, and the solution was stirred at room temperature for $3 \mathrm{~h}$. The resulting mixture was filtered through a small pad of silica gel. The filtrate was concentrated in vacuo and purified by gel column chromatography $\left(\mathrm{R}_{f}=0.4\right.$, 15\% Ethyl acetate/Hexane) to give $\mathbf{3 b a - 1}$ as white solid (20 mg, 67\%).

Butyl (E)-3-(3-(3-oxopropyl)-1H-indol-7-yl)acrylate (3ba-1)

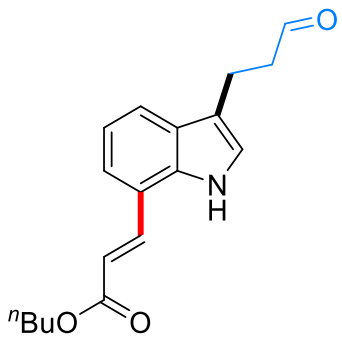

mp 94-96 ${ }^{\circ} \mathrm{C} ;{ }^{1} \mathrm{H}$ NMR $\left(400 \mathrm{MHz}, \mathrm{CDCl}_{3}\right) \delta 9.85(\mathrm{t}, J=1.5 \mathrm{~Hz}, 1 \mathrm{H}), 8.47$ (bs, 1H), 8.00 (d, $J=16.0 \mathrm{~Hz}$, $1 \mathrm{H}), 7.64(\mathrm{~d}, J=7.8 \mathrm{~Hz}, 1 \mathrm{H}), 7.43(\mathrm{~d}, J=7.4 \mathrm{~Hz}, 1 \mathrm{H}), 7.16(\mathrm{t}, J=7.6 \mathrm{~Hz}, 1 \mathrm{H}), 7.05-7.06(\mathrm{~m}, 1 \mathrm{H}), 6.50$ (d, $J=16.0 \mathrm{~Hz}, 1 \mathrm{H}), 4.24(\mathrm{t}, J=6.7 \mathrm{~Hz}, 2 \mathrm{H}), 3.12(\mathrm{t}, J=7.3 \mathrm{~Hz}, 2 \mathrm{H}), 2.84-2.88(\mathrm{~m}, 2 \mathrm{H}), 1.68-1.75(\mathrm{~m}, 2 \mathrm{H})$, $1.41-1.50(\mathrm{~m}, 2 \mathrm{H}), 0.98(\mathrm{t}, J=7.4 \mathrm{~Hz}, 3 \mathrm{H}) ;{ }^{13} \mathrm{C} \mathrm{NMR}\left(100 \mathrm{MHz}, \mathrm{CDCl}_{3}\right) \delta 202.1,167.3,140.9,134.8$, 128.2, 122.6, 122.1, 121.1, 119.8, 118.4, 118.2, 115.4, 64.6, 43.9, 30.8, 19.2, 17.6, 13.7; HRMS m/z: $[\mathrm{M}+\mathrm{H}]^{+}$Calcd for $\mathrm{C}_{18} \mathrm{H}_{22} \mathrm{NO}_{3}$ 300.1594; Found 300.1594.

\section{4-5. Base-mediated Michael addition to 2-benzylidenemalononitrile (Scheme 5)}

To an oven dried Schlenk tube equipped with a magnetic stirrer bar were added 3ba (25 mg, $0.1 \mathrm{mmol}), 2$ benzylidenemalononitrile (16 mg, $0.1 \mathrm{mmol})$, and $\mathrm{CH}_{3} \mathrm{CN}(1.5 \mathrm{~mL}) . \mathrm{KOH}$ (powder) $(5.8 \mathrm{mg}, 0.1 \mathrm{mmol})$ was added to the tube, and the solution was stirred at room temperature for $3 \mathrm{~h}$. The resulting mixture was extracted with EtOAc, and the extract was washed with brine. The organic phase was dried over $\mathrm{Na}_{2} \mathrm{SO}_{4}$, concentrated in vacuo, and purified by silica gel column chromatography $\left(\mathrm{R}_{f}=0.4\right.$, $15 \%$ Ethyl acetate/Hexane) to give $\mathbf{3 b a - 2}$ as pale yellow gum (25 mg, 61\%). 
Butyl (E)-3-(3-(2,2-dicyano-1-phenylethyl)-1H-indol-7-yl)acrylate (3ba-2)

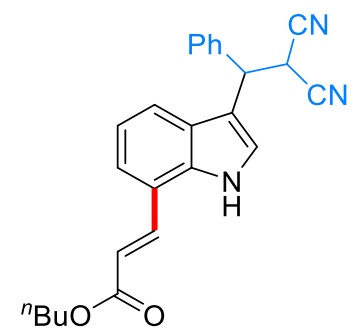

${ }^{1} \mathrm{H}$ NMR (400 MHz, $\left.\mathrm{CDCl}_{3}\right) \delta 8.96$ (bs, $\left.1 \mathrm{H}\right), 8.01(\mathrm{~d}, J=16.0 \mathrm{~Hz}, 1 \mathrm{H}), 7.35-7.45(\mathrm{~m}, 7 \mathrm{H}), 7.32(\mathrm{~d}, J=8.0$ $\mathrm{Hz}, 1 \mathrm{H}), 7.08(\mathrm{t}, J=8.0 \mathrm{~Hz}, 1 \mathrm{H}), 6.52(\mathrm{~d}, J=16.0 \mathrm{~Hz}, 1 \mathrm{H}), 4.94(\mathrm{~d}, J=6.4 \mathrm{~Hz}, 1 \mathrm{H}), 4.49$ (d, $J=6.4 \mathrm{~Hz}$, $1 \mathrm{H}), 4.26(\mathrm{t}, J=6.7 \mathrm{~Hz}, 2 \mathrm{H}), 1.68-1.75(\mathrm{~m}, 2 \mathrm{H}), 1.41-1.50(\mathrm{~m}, 2 \mathrm{H}), 0.9(\mathrm{t}, J=7.4 \mathrm{~Hz}, 3 \mathrm{H}) ;{ }^{13} \mathrm{C} \mathrm{NMR}(100$ $\left.\mathrm{MHz}, \mathrm{CDCl}_{3}\right) \delta 167.3,140.1,136.9,134.8,129.2,128.8,128.1,126.9,122.8,122.75,121.1,120.6,118.8$, 113.0, 112.2, 112.1, 64.7, 44.0, 30.7, 29.6, 19.2, 13.7; HRMS m/z: $[\mathrm{M}+\mathrm{H}]^{+}$Calcd for $\mathrm{C}_{25} \mathrm{H}_{24} \mathrm{~N}_{3} \mathrm{O}_{2} 398.1863$; Found 398.1862.

\section{4-6. Intramolecular cyclization (Scheme 5)}

To a round-bottom flask equipped with a magnetic stirrer bar were $3 \mathbf{b a}(25 \mathrm{mg}, 0.1 \mathrm{mmol}), \mathrm{MeOH}(5.0$ $\mathrm{mL}$ ), and $\mathrm{K}_{2} \mathrm{CO}_{3}(42 \mathrm{mg}, 0.3 \mathrm{mmol})$. The solution stirred at $50{ }^{\circ} \mathrm{C}$ for $12 \mathrm{~h}$ with an oil bath. The resulting mixture was filtered through a pad of silica gel. The filtrate was concentrated in vacuo, and the residue was purified by chromatography $\left(\mathrm{R}_{f}=0.4,20 \%\right.$ Ethyl acetate/Hexane) to give $\mathbf{3 b a - 3}$ as white solid ( $\left.8 \mathrm{mg}, 45 \%\right)$. The ${ }^{1} \mathrm{H}$ NMR data was identical to one reported in a literature.

4H-Pyrrolo[3,2,1-ij]quinolin-4-one (3ba-3) ${ }^{4}$<smiles></smiles>

${ }^{1} \mathrm{H}$ NMR $\left(400 \mathrm{MHz}, \mathrm{CDCl}_{3}\right) \delta 7.97(\mathrm{~d}, J=3.6 \mathrm{~Hz}, 1 \mathrm{H}), 7.84-7.87(\mathrm{~m}, 2 \mathrm{H}), 7.62(\mathrm{~d}, J=7.6 \mathrm{~Hz}, 1 \mathrm{H}), 7.44$ $(\mathrm{t}, J=7.6 \mathrm{~Hz}, 1 \mathrm{H}), 6.90(\mathrm{~d}, J=3.6 \mathrm{~Hz}, 1 \mathrm{H}), 6.73(\mathrm{~d}, J=9.5 \mathrm{~Hz}, 1 \mathrm{H})$; HRMS $m / z:[\mathrm{M}+\mathrm{H}]^{+}$Calcd for $\mathrm{C}_{11} \mathrm{H}_{8} \mathrm{NO}$ 170.0600; found 170.0605 .

\section{4-7. Synthesis of oxoindoline (Scheme 5)}

To an oven dried Schlenk tube equipped with a magnetic bar were added $3 \mathbf{k a}$ ( $26 \mathrm{mg}, 0.1 \mathrm{mmol}), \mathrm{KBr}(1.2$ $\mathrm{mg}, 10 \mathrm{~mol} \%)$, tert- $\mathrm{BuOH} / \mathrm{H}_{2} \mathrm{O}(20: 1 \mathrm{v} / \mathrm{v}, 1.0 \mathrm{~mL})$, and oxone $(74.5 \mathrm{mg}, 0.12 \mathrm{mmol})$. The mixture was stirred at room temperature for $2.5 \mathrm{~h}$. The resulting suspension was poured into saturated aqueous $\mathrm{Na}_{2} \mathrm{SO}_{3}$ 
and extracted with EtOAc. The organic layer was dried over $\mathrm{Na}_{2} \mathrm{SO}_{4}$, concentrated in vacuo, and purified by silica gel column chromatography $\left(\mathrm{R}_{f}=0.3,30 \%\right.$ Ethyl acetate/Hexane) to give $\mathbf{3 k a - 1}$ as white solid (25 $\mathrm{mg}, 91 \%)$.

Butyl (E)-3-(3-methyl-2-oxoindolin-7-yl)acrylate (3ka-1)

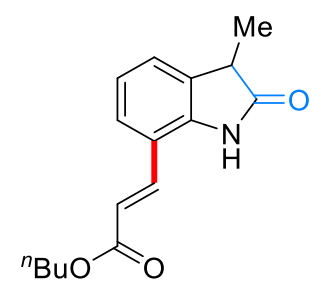

mp 101-103 ${ }^{\circ} \mathrm{C} ;{ }^{1} \mathrm{H}$ NMR $\left(400 \mathrm{MHz}, \mathrm{CDCl}_{3}\right) \delta 9.35$ (bs, $\left.1 \mathrm{H}\right), 7.89(\mathrm{~d}, J=15.8 \mathrm{~Hz}, 1 \mathrm{H}), 7.43(\mathrm{~d}, J=8.1$ $\mathrm{Hz}, 1 \mathrm{H}), 7.22(\mathrm{~d}, J=7.3 \mathrm{~Hz}, 1 \mathrm{H}), 7.04(\mathrm{t}, J=7.6 \mathrm{~Hz}, 1 \mathrm{H}), 6.43(\mathrm{~d}, J=15.8 \mathrm{~Hz}, 1 \mathrm{H}), 4.25(\mathrm{t}, J=6.7 \mathrm{~Hz}$, 2H), $3.49(\mathrm{~d}, J=7.7 \mathrm{~Hz}, 1 \mathrm{H}), 1.66-1.73(\mathrm{~m}, 2 \mathrm{H}), 1.52$ (d, $J=7.7 \mathrm{~Hz}, 3 \mathrm{H}), 1.40-1.49(\mathrm{~m}, 2 \mathrm{H}), 0.96(\mathrm{t}, J=$ $7.4 \mathrm{~Hz}, 3 \mathrm{H}) ;{ }^{13} \mathrm{C}$ NMR $\left(100 \mathrm{MHz}, \mathrm{CDCl}_{3}\right) \delta 180.8,167.1,141.0,138.7,132.4,125.5,125.3,122.5,119.1$, 116.9, 64.7, 40.8, 30.7, 19.2, 15.2, 13.7; HRMS m/z: $[\mathrm{M}+\mathrm{H}]^{+}$Calcd for $\mathrm{C}_{16} \mathrm{H}_{20} \mathrm{NO}_{3}$ 274.1438; Found 274.1440 .

\section{4-8. Light-induced $[2+2]$ cyclization (Scheme 5)}

Asahi Spectra MAX-303 compact xenon lamp (300 W, white light: 300 600 nm) was used as the light source. The powder of $\mathbf{3 b a}(25 \mathrm{mg}, 0.1 \mathrm{mmol})$ was exposed to the light at room temperature for $17 \mathrm{~h}$, producing the cycloadduct 3ba-4 as a single isomer in $80 \%$ NMR yield. This compound was isolated by GPC (EtOAc) as white solid (20 mg, 77\%). Single crystals suitable for the X-ray analysis were obtained by slow evaporation from the $n$-hexane solution.

Dibutyl 2,4-di(1H-indol-7-yl)cyclobutane-1,3-dicarboxylate (3ba-4)

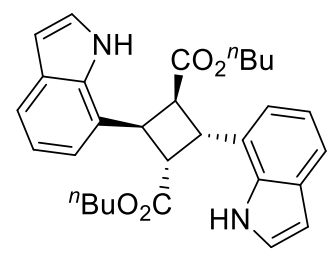

$\left(\mathrm{R}_{f}=0.3,20 \%\right.$ Ethyl acetate/Hexane); mp 229-231 ${ }^{\circ} \mathrm{C} ;{ }^{1} \mathrm{H}$ NMR (400 MHz, $\left.\mathrm{CDCl}_{3}\right) \delta 8.60$ (bs, $\left.2 \mathrm{H}\right), 7.57$ $(\mathrm{d}, J=7.8 \mathrm{~Hz}, 2 \mathrm{H}), 7.21-7.23(\mathrm{~m}, 4 \mathrm{H}), 7.12(\mathrm{t}, J=7.6 \mathrm{~Hz}, 2 \mathrm{H}), 6.56(\mathrm{dd}, J=3.2,2.0 \mathrm{~Hz}, 2 \mathrm{H}), 4.86(\mathrm{dd}, J$ $=10.2 \mathrm{~Hz}, 7.1 \mathrm{~Hz}, 2 \mathrm{H}), 4.28(\mathrm{dd}, J=10.2,7.1 \mathrm{~Hz}, 2 \mathrm{H}), 3.63-3.69(\mathrm{~m}, 2 \mathrm{H}), 3.45-3.51(\mathrm{~m}, 2 \mathrm{H}), 0.83-0.97$ (m, 8H), 0.66-0.70 (m, 6H); ${ }^{13} \mathrm{C}$ NMR (100 MHz, $\left.\mathrm{CDCl}_{3}\right) \delta 172.7,134.8,128.1,124.1,121.3,120.0,119.8$, 
119.0, 103.1, 64.7, 45.7, 37.3, 29.9, 18.7, 13.5; HRMS m/z: $[\mathrm{M}+\mathrm{H}]^{+}$Calcd for $\mathrm{C}_{30} \mathrm{H}_{35} \mathrm{~N}_{2} \mathrm{O}_{4}$ 487.2591; Found 487.2586 .

On the other hand, when the chloroform $(3.0 \mathrm{~mL})$ solution of $\mathbf{3 b a}(25 \mathrm{mg}, 0.1 \mathrm{mmol})$ was exposed to the light at room temperature for $6 \mathrm{~h}$, only the corresponding $Z$ isomer $\mathbf{3 b a}-\mathbf{5}$ formed, whereas the dimer $\mathbf{3 b a}$ 4 was not detected. The $Z$ isomer 3 ba-5 was isolated by GPC (EtOAc) as pale yellow gum (13 mg, 50\%).

Butyl (Z)-3-(1H-indol-7-yl)acrylate (3ba-5)

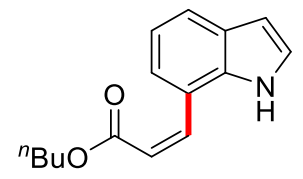

$\left(\mathrm{R}_{f}=0.4,20 \%\right.$ Ethyl acetate/Hexane); ${ }^{1} \mathrm{H}$ NMR (400 MHz, $\left.\mathrm{CDCl}_{3}\right) \delta 8.60$ (bs, $\left.1 \mathrm{H}\right), 7.64(\mathrm{~d}, J=7.9 \mathrm{~Hz}$, $1 \mathrm{H}), 7.30(\mathrm{dt}, J=7.4,1.0 \mathrm{~Hz}, 1 \mathrm{H}), 7.20-7.23(\mathrm{~m}, 2 \mathrm{H}), 7.11(\mathrm{t}, J=7.6 \mathrm{~Hz}, 1 \mathrm{H}), 6.57(\mathrm{dd}, J=3.2,2.0 \mathrm{~Hz}$, $1 \mathrm{H}), 6.12(\mathrm{~d}, J=12.5 \mathrm{~Hz}, 1 \mathrm{H}), 4.03(\mathrm{t}, J=6.7 \mathrm{~Hz}, 2 \mathrm{H}), 1.38-1.45(\mathrm{~m}, 2 \mathrm{H}), 1.11-1.20(\mathrm{~m}, 2 \mathrm{H}), 0.82(\mathrm{t}, J=$ $7.4 \mathrm{~Hz}, 3 \mathrm{H}) ;{ }^{13} \mathrm{C} \mathrm{NMR}\left(100 \mathrm{MHz}, \mathrm{CDCl}_{3}\right) \delta 166.8,139.3,133.9,128.3,124.3,123.8,122.0,120.9,119.4$, 119.1, 103.1, 64.5, 30.3, 19.0, 13.6; HRMS m/z: $[\mathrm{M}+\mathrm{H}]^{+}$Calcd for $\mathrm{C}_{15} \mathrm{H}_{18} \mathrm{NO}_{2}$ 244.1332; Found 244.1327. 


\section{X-ray crystallographic analysis}

Single crystals of 3ka and 3ba-4 suitable for X-ray analysis were obtained by slow evaporation from $n$ hexane solutions. The structures were refined by full-matrix least-squares method using SHELXL-2016/6. ${ }^{5}$ Hydrogen atoms were included in the refinement on calculated positions riding on their carrier atoms. ORTEP-3 programs were used to draw the molecules. ${ }^{6}$ These data can be obtained free of charge from the Cambridge Crystallographic Data Centre via www.ccdc.cam.ac.uk/data_request/cif.

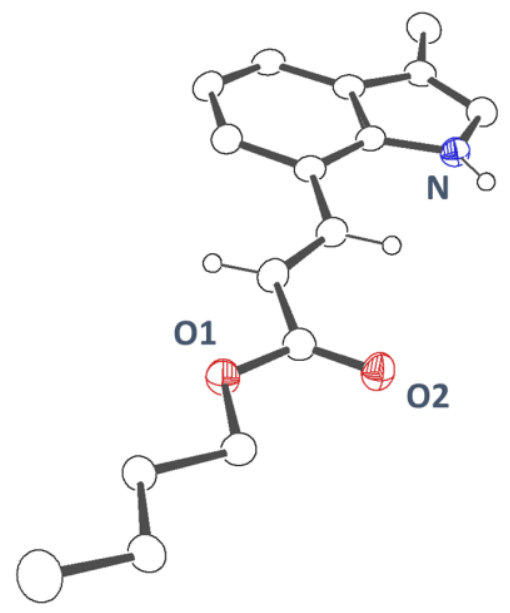

Figure S1. ORTEP drawing for 3ka with $40 \%$ thermal ellipsoid. Hydrogen atoms of C-H bonds within indole rings and alkyl groups are omitted for clarity.

Table S2. Crystal data for $3 \mathbf{k a}$

CCDC No.

2089543

Crystal system

triclinic

Space group

P-1 (No. 2)

Unit cell parameter $[\AA$, deg]

$a=7.5705(5), \alpha=94.436(4)$

$b=8.4949(4), \beta=101.818(5)$

$c=11.6454(6), \gamma=107.239(5)$

Cell volume $\left[\AA^{3}\right]$

692.44(7)

Z

2

$\mathrm{R}$ factor $(I>2.0 \sigma(I))$

$R 1=0.0495, w R 2=0.1346$

$\mathrm{R}$ factor (all data)

$R 1=0.0602, w R 2=0.1435$

Rint

0.0514

Goodness of fit

1.071 


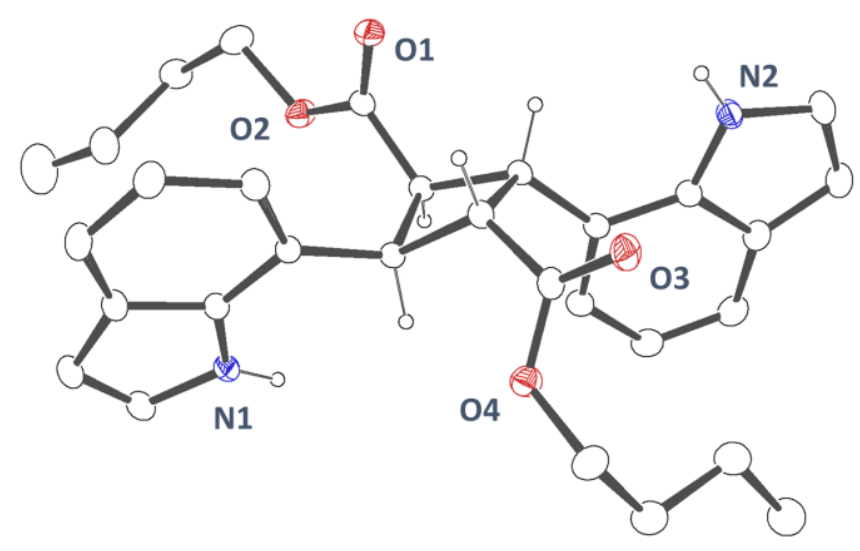

Figure S2. ORTEP drawing for 3ba-4 with 30\% thermal ellipsoid. Hydrogen atoms of C-H bonds within indole rings and $n$-butyl groups are omitted for clarity.

Table S3. Crystal data for 3ba-4

CCDC No.

Crystal system

Space group

Unit cell parameter $[\AA$, deg]

Cell volume $\left[\AA^{3}\right]$

$\mathrm{Z}$

$\mathrm{R}$ factor $(I>2.0 \sigma(I))$

$\mathrm{R}$ factor (all data)

Rint

Goodness of fit
2089544

monoclinic

$P 2{ }_{1} / c$ (No. 14)

$a=13.9839(2)$

$b=12.1482(2), \beta=105.1500(10)$

$c=15.9164(2)$

2609.89(7)

4

$R 1=0.0528, w R 2=0.1444$

$R 1=0.0584, w R 2=0.1500$

0.0374

1.067 

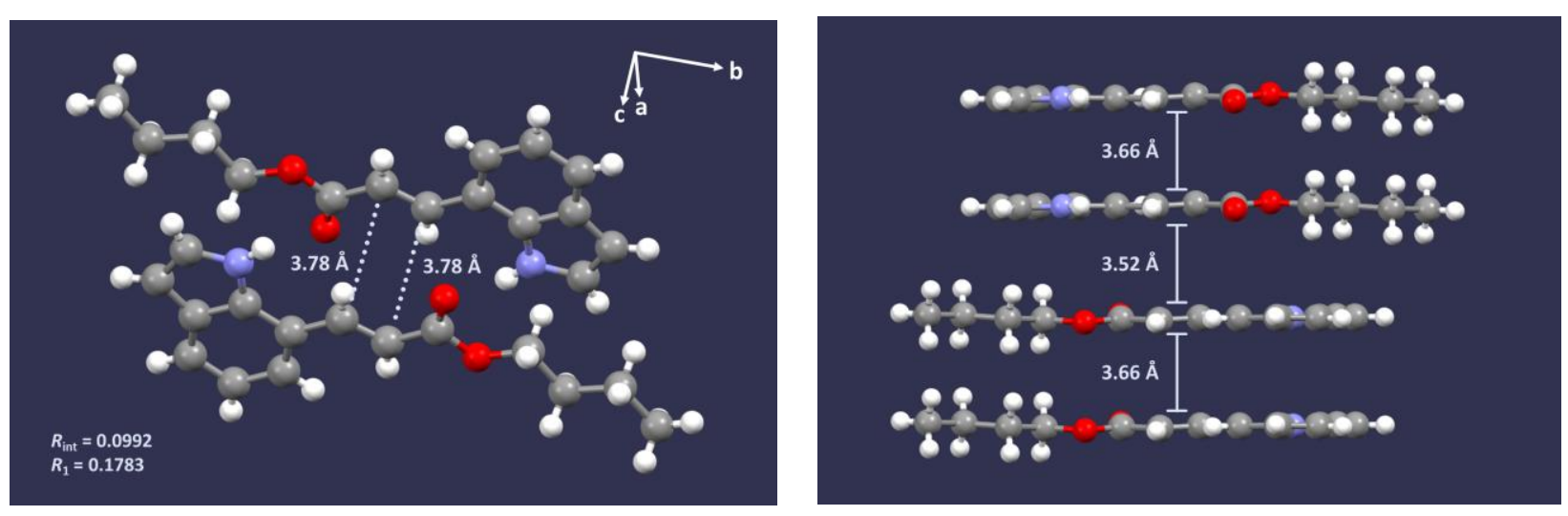

Figure S3. A crystal packing model for the compound $\mathbf{3 b a}$ in a preliminary crystallographic analysis $($ carbon $=$ gray, hydrogen $=$ white, oxygen $=$ red, nitrogen $=$ blue $)$. 


\section{Computation study}

The Hirshfeld population analysis was conducted at the B3LYP/6-311++G(3df,2pd)//B3LYP/6-31+G(d,p) level using the Gaussian 16 program. $^{7}$ The optimized molecular structures were verified by vibrational analysis; equilibrium structures did not have imaginary frequencies.
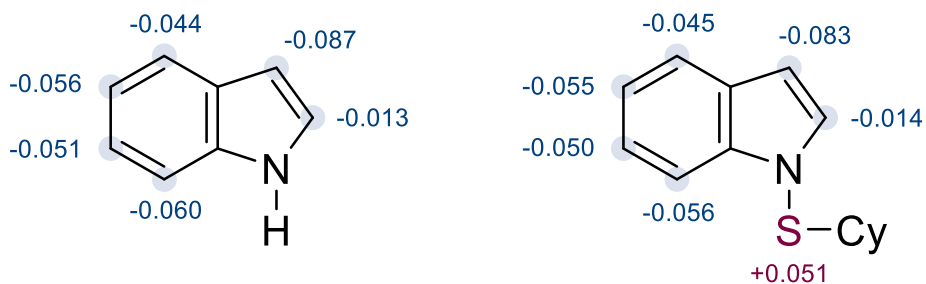

Figure S4. Summary of the calculated Hirshfeld charge (elementary charge).

Table S4. Cartesian coordinates for the optimized structure of indole

\begin{tabular}{|l|l|l|l|}
\hline C & 0.248458 & -0.67233 & 0.000123 \\
\hline C & 0.24987 & 0.751732 & 0.00006 \\
\hline C & -0.98363 & 1.430471 & 0.000125 \\
\hline C & -2.16165 & 0.692424 & $-5.4 \mathrm{E}-05$ \\
\hline C & -2.13773 & -0.71989 & -0.00038 \\
\hline C & -0.93539 & -1.42032 & 0.000319 \\
\hline C & 1.626518 & 1.16848 & -0.00009 \\
\hline H & -1.01264 & 2.516651 & 0.000523 \\
\hline H & -3.11843 & 1.20634 & 0.000364 \\
\hline H & -3.07457 & -1.26924 & -0.00075 \\
\hline H & -0.91957 & -2.50686 & 0.000287 \\
\hline H & 2.000266 & 2.182686 & $-5.4 \mathrm{E}-05$ \\
\hline C & 2.392707 & 0.030273 & -0.00012 \\
\hline N & 1.568429 & -1.08174 & $-9.6 \mathrm{E}-05$ \\
\hline H & 1.883688 & -2.03838 & 0.000738 \\
\hline H & 3.467333 & -0.0841 & -0.00038 \\
\hline & & & \\
\hline
\end{tabular}


Table S5. Cartesian coordinates for the optimized structure of $\mathbf{1 b}$

\begin{tabular}{|c|c|c|c|}
\hline C & 1.83534 & 0.084026 & -0.28209 \\
\hline C & 2.870042 & 0.441712 & 0.624889 \\
\hline C & 3.986357 & -0.4048 & 0.752553 \\
\hline C & 4.047903 & -1.5621 & -0.0163 \\
\hline C & 3.012412 & -1.89154 & -0.91667 \\
\hline C & 1.893938 & -1.07459 & -1.0625 \\
\hline C & 2.488164 & 1.690169 & 1.231939 \\
\hline $\mathrm{H}$ & 4.789137 & -0.15364 & 1.440415 \\
\hline $\mathrm{H}$ & 4.905838 & -2.2222 & 0.072121 \\
\hline $\mathrm{H}$ & 3.089551 & -2.79789 & -1.51009 \\
\hline $\mathrm{H}$ & 1.099163 & -1.32067 & -1.75868 \\
\hline $\mathrm{H}$ & 3.047247 & 2.253772 & 1.966031 \\
\hline C & 1.281035 & 2.047783 & 0.698281 \\
\hline$N$ & 0.858158 & 1.080476 & -0.21536 \\
\hline $\mathrm{H}$ & 0.664704 & 2.917581 & 0.873621 \\
\hline$S$ & -0.57116 & 1.179815 & -1.15913 \\
\hline C & -1.86134 & 0.478142 & -0.02343 \\
\hline C & -3.2192 & 0.687335 & -0.7155 \\
\hline C & -1.63205 & -0.9949 & 0.334764 \\
\hline $\mathrm{H}$ & -1.82928 & 1.087924 & 0.888316 \\
\hline C & -4.3658 & 0.145417 & 0.158511 \\
\hline $\mathrm{H}$ & -3.21945 & 0.160565 & -1.68056 \\
\hline $\mathrm{H}$ & -3.3777 & 1.750599 & -0.93057 \\
\hline C & -2.77709 & -1.52667 & 1.21622 \\
\hline $\mathrm{H}$ & -1.57892 & -1.58156 & -0.59237 \\
\hline $\mathrm{H}$ & -0.67079 & -1.11535 & 0.844489 \\
\hline C & -4.1444 & -1.32496 & 0.545238 \\
\hline $\mathrm{H}$ & -5.3172 & 0.263138 & -0.37392 \\
\hline $\mathrm{H}$ & -4.44053 & 0.754357 & 1.070654 \\
\hline $\mathrm{H}$ & -2.60964 & -2.58825 & 1.433752 \\
\hline $\mathrm{H}$ & -2.76473 & -1.0051 & 2.184114 \\
\hline $\mathrm{H}$ & -4.94853 & -1.66468 & 1.209101 \\
\hline $\mathrm{H}$ & -4.19933 & -1.94918 & -0.35835 \\
\hline
\end{tabular}




\section{Copy of NMR Spectra}

${ }^{1} \mathrm{H}$ NMR of $\mathbf{1 b}\left(400 \mathrm{MHz}, \mathrm{CDCl}_{3}\right)$

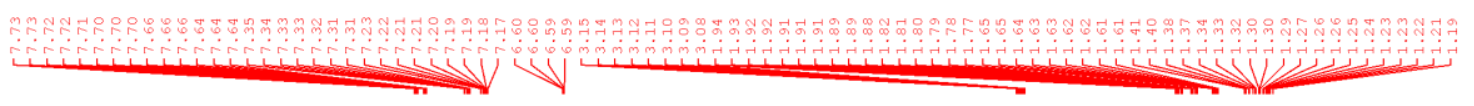<smiles>c1ccc2c(c1)ccn2SC1CCCC1</smiles>

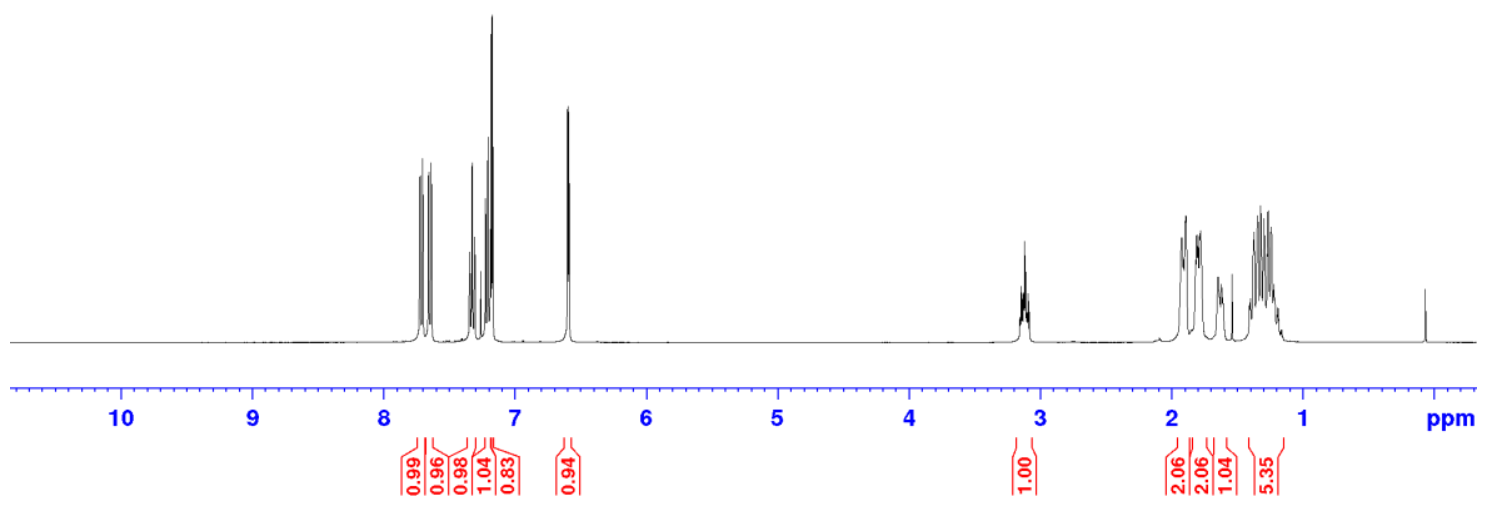

${ }^{13} \mathrm{C}$ NMR of $\mathbf{1 b}\left(100 \mathrm{MHz}, \mathrm{CDCl}_{3}\right)$
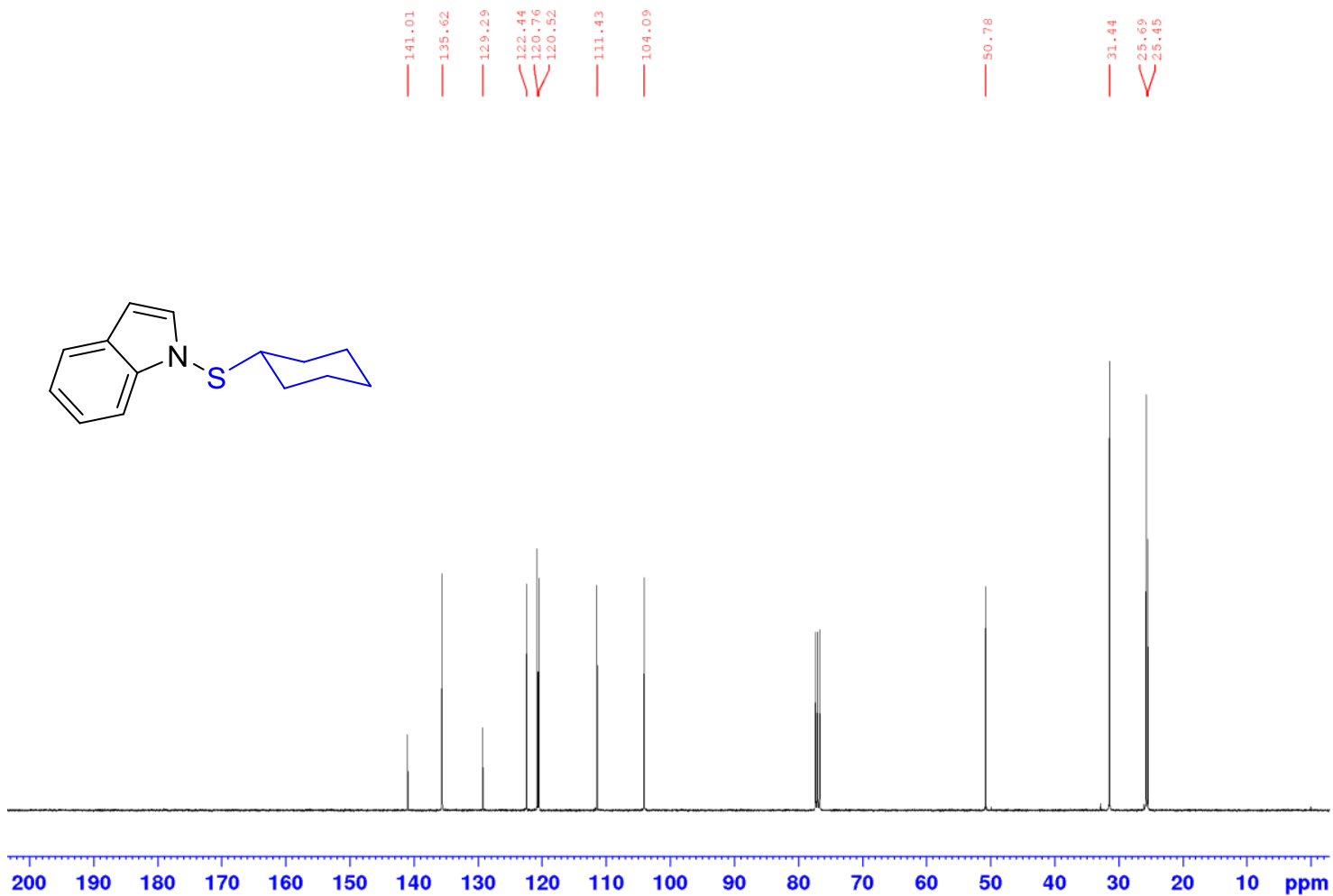
${ }^{1} \mathrm{H}$ NMR of $1 \mathrm{c}\left(400 \mathrm{MHz}, \mathrm{CDCl}_{3}\right)$

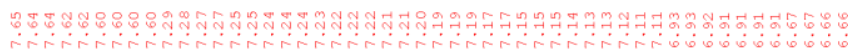

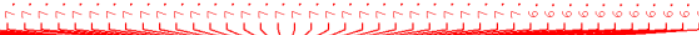
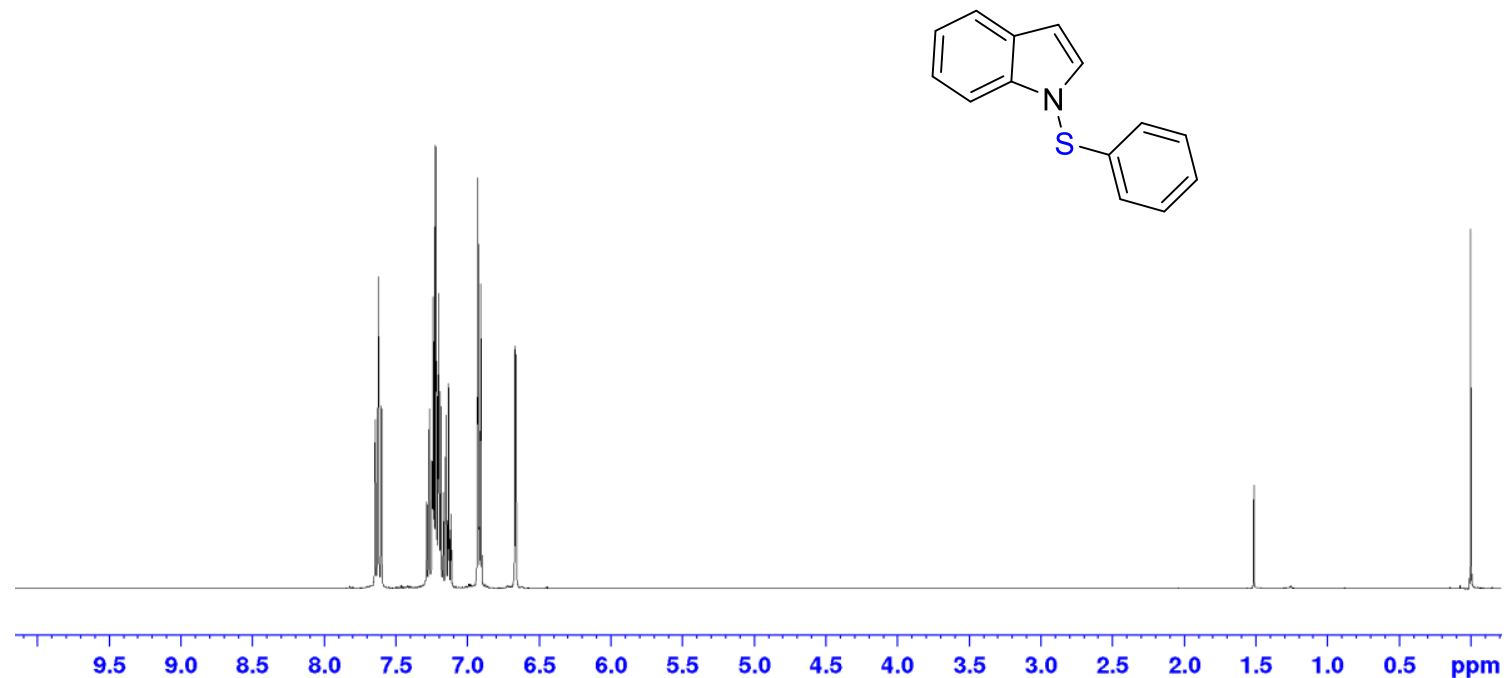

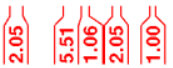

${ }^{13} \mathrm{C}$ NMR of $1 \mathbf{c}\left(100 \mathrm{MHz}, \mathrm{CDCl}_{3}\right)$
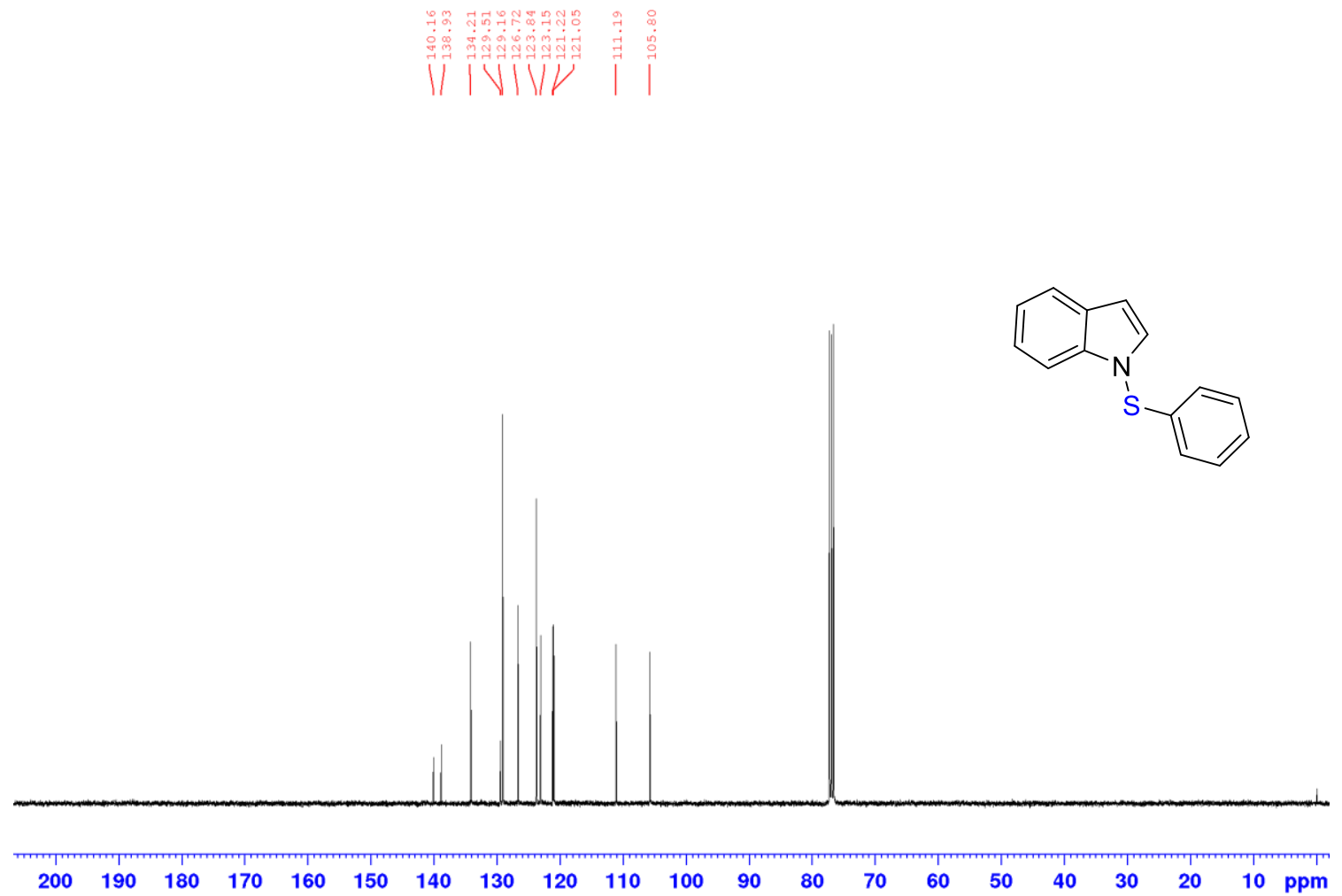
${ }^{1} \mathrm{H}$ NMR of $1 \mathbf{d}\left(400 \mathrm{MHz}, \mathrm{CDCl}_{3}\right)$

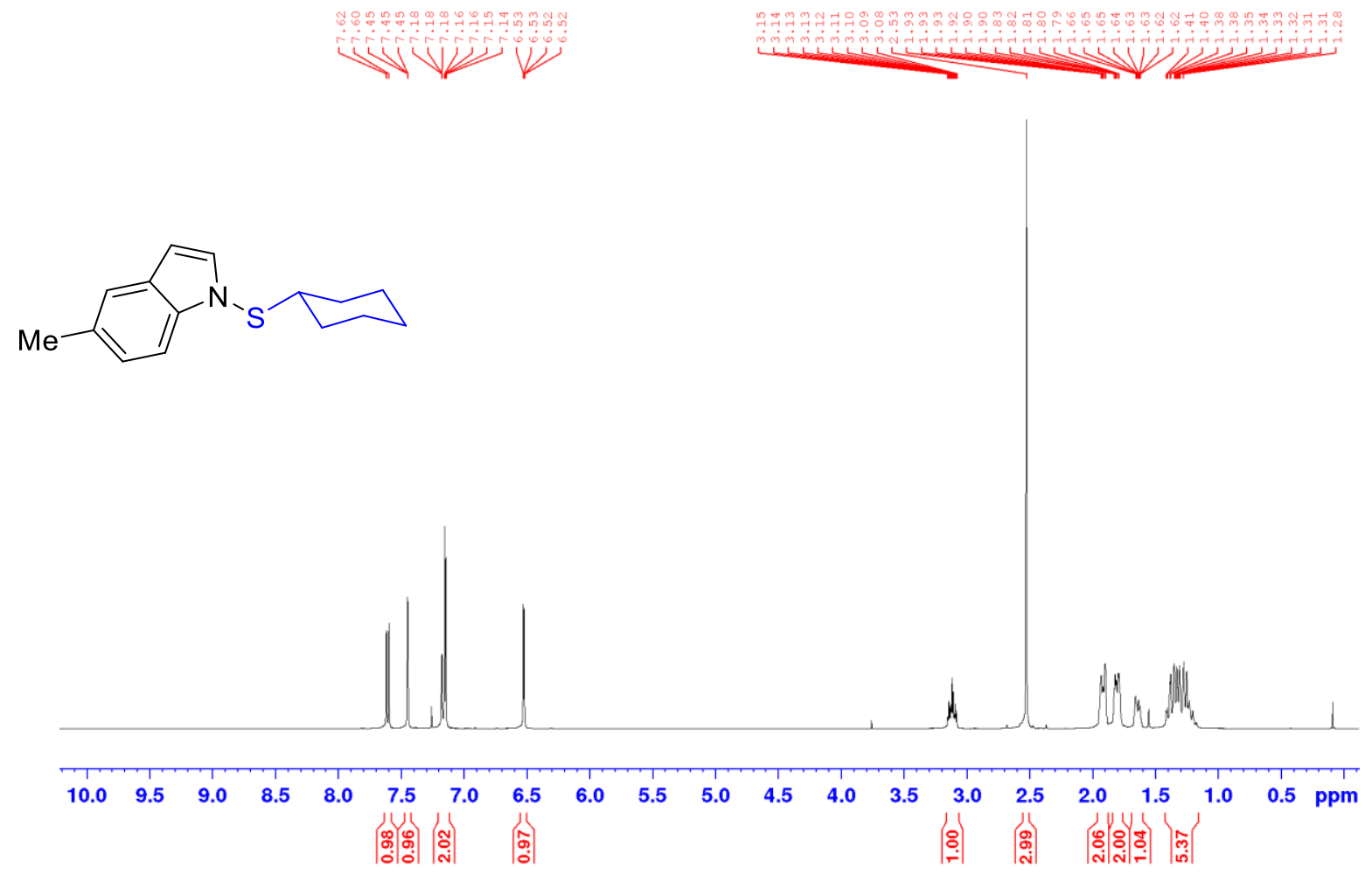

${ }^{13} \mathrm{C} \mathrm{NMR}$ of $1 d\left(100 \mathrm{MHz}, \mathrm{CDCl}_{3}\right)$

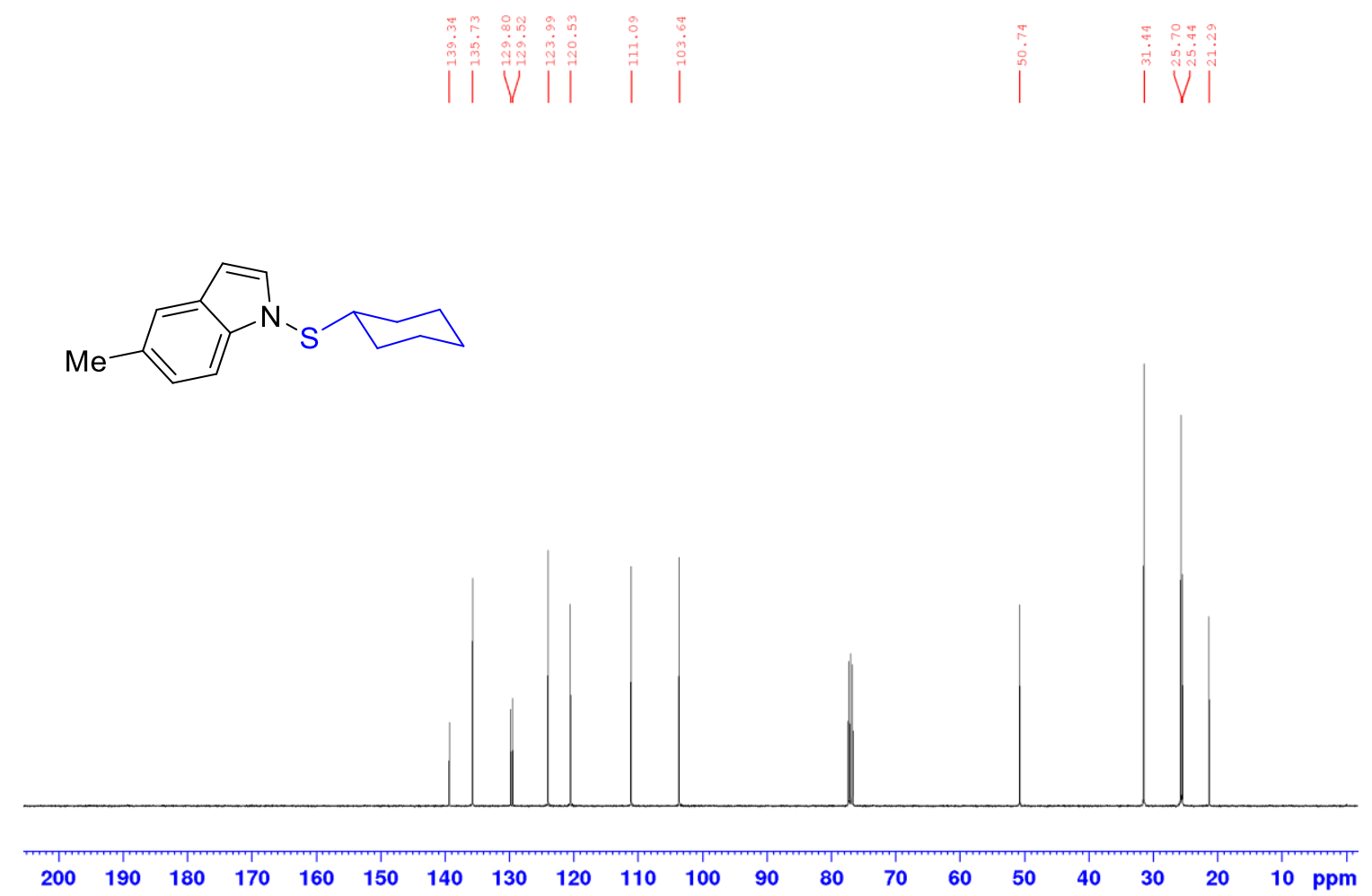


${ }^{1} \mathrm{H}$ NMR of $1 e\left(400 \mathrm{MHz}, \mathrm{CDCl}_{3}\right)$

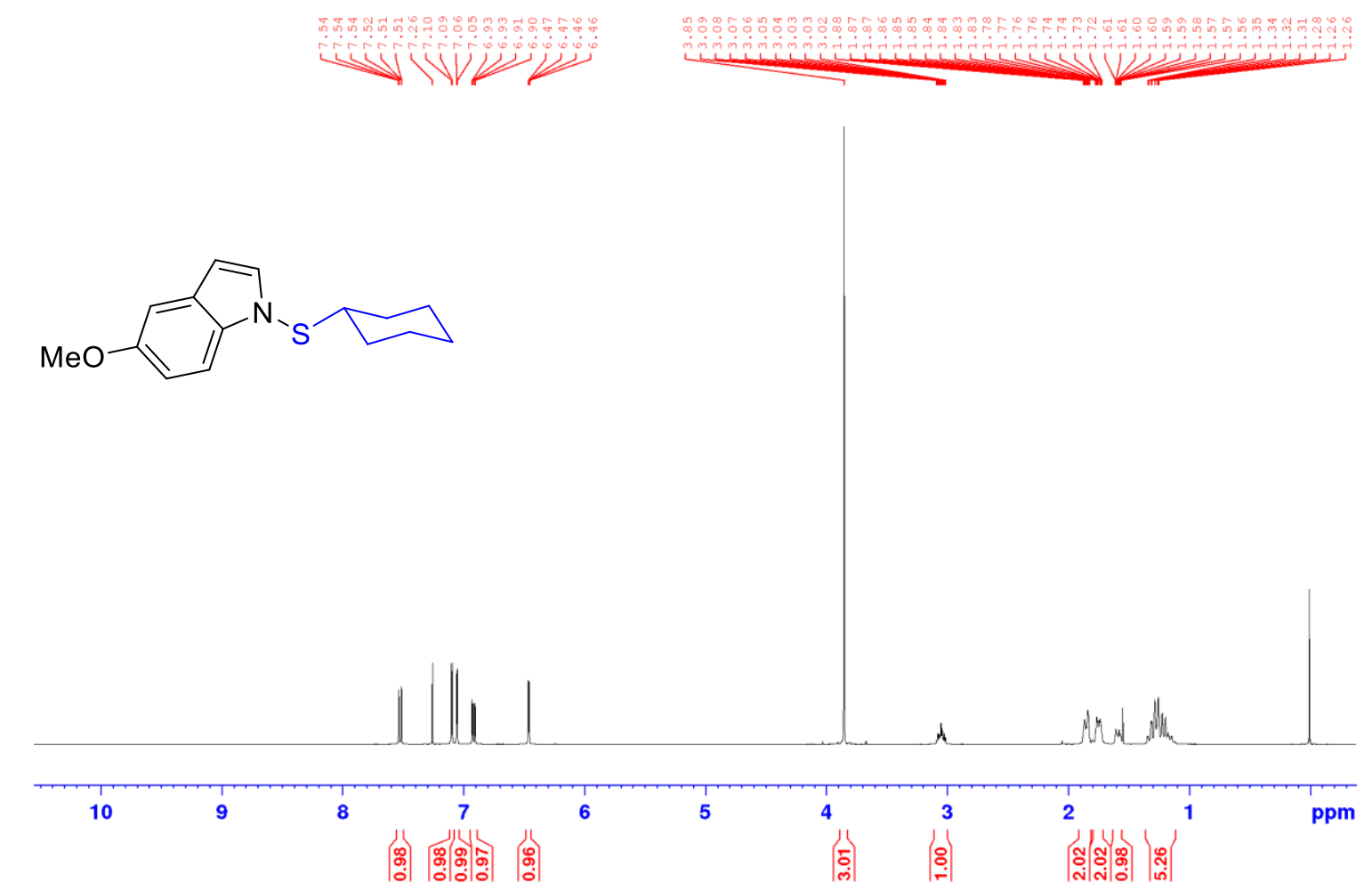

${ }^{13} \mathrm{C}$ NMR of 1 e $\left(100 \mathrm{MHz}, \mathrm{CDCl}_{3}\right)$
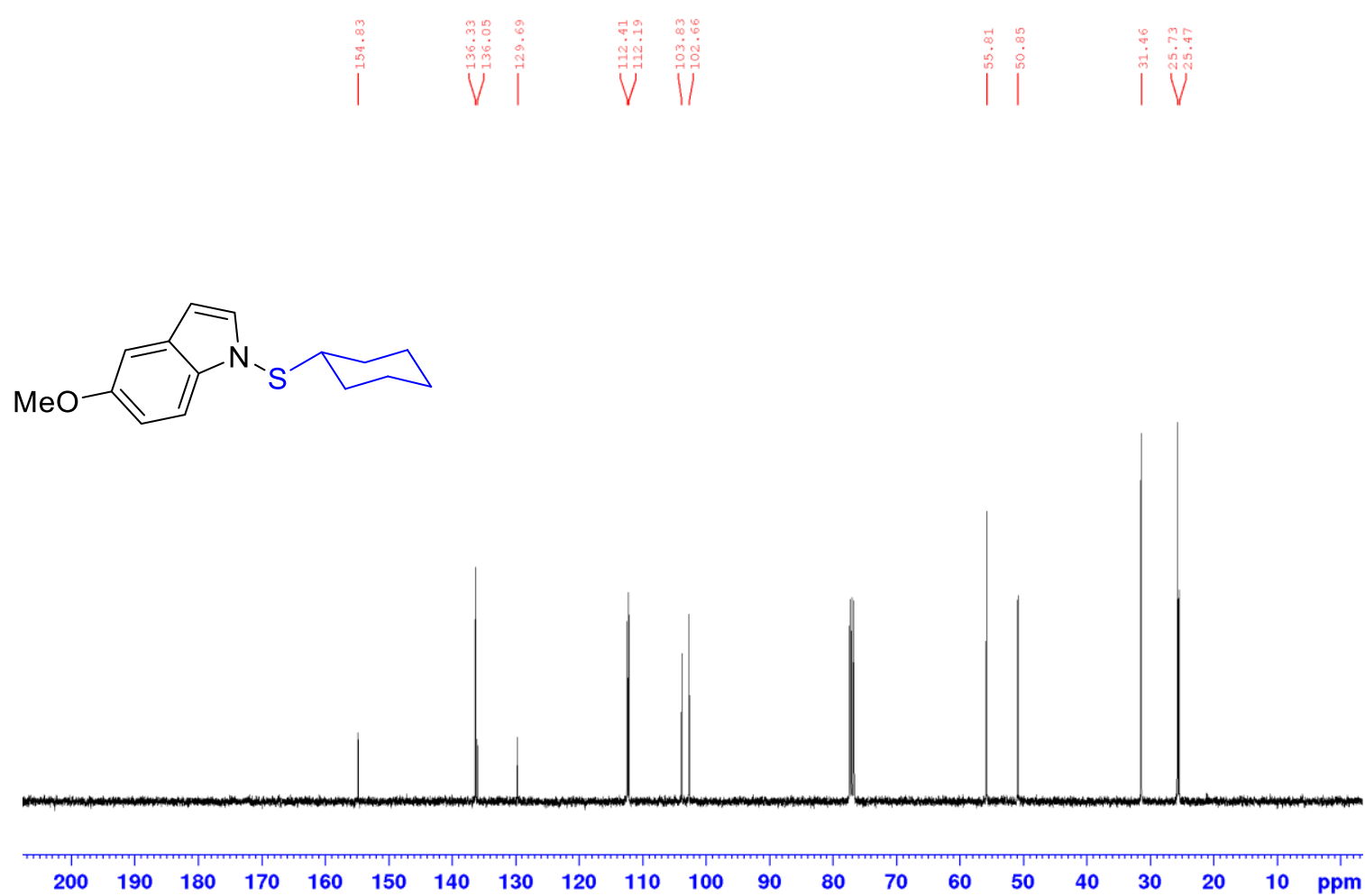
${ }^{1} \mathrm{H}$ NMR of $\mathbf{1 f}\left(400 \mathrm{MHz}, \mathrm{CDCl}_{3}\right)$

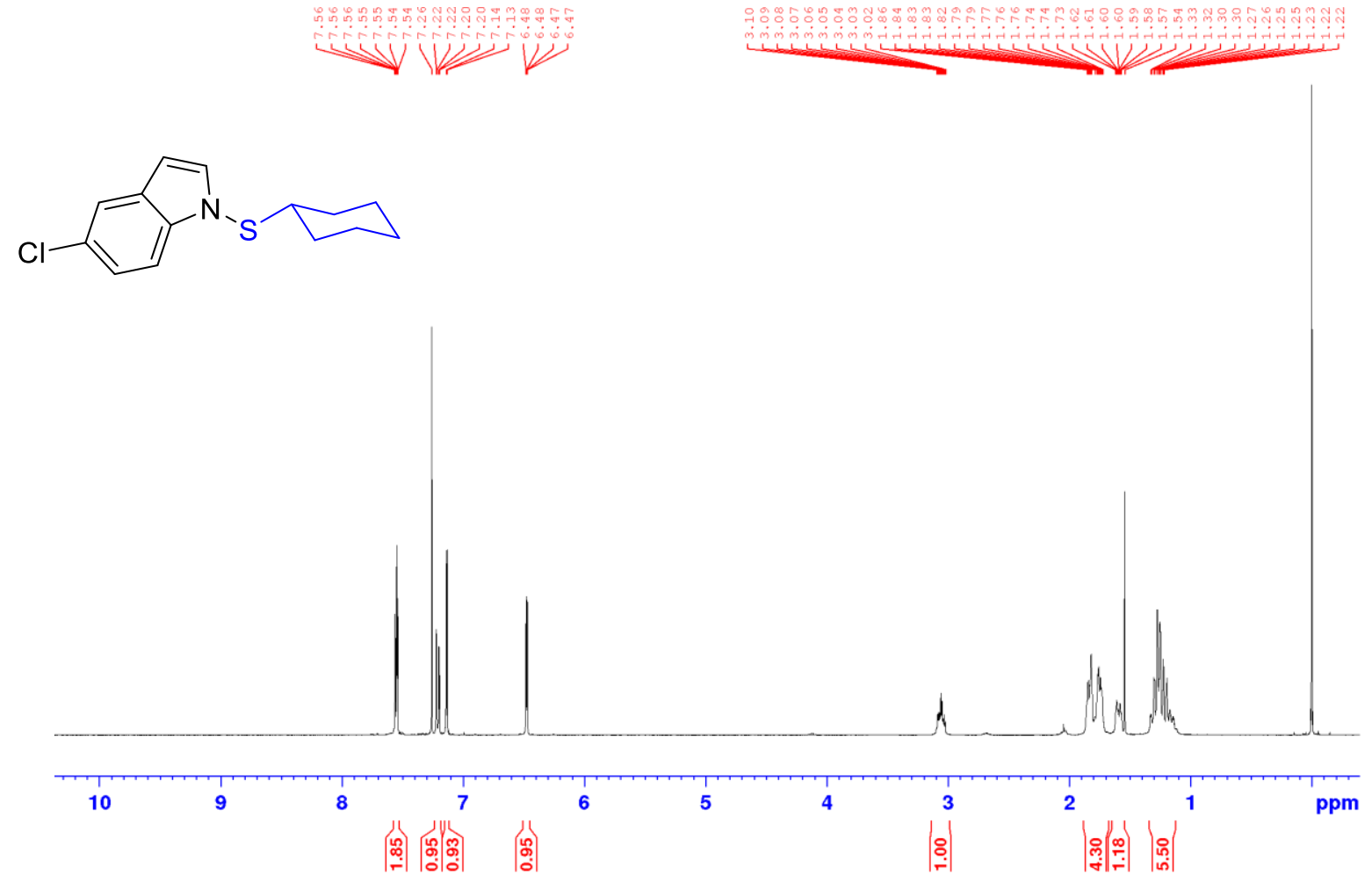

${ }^{13} \mathrm{C}$ NMR of $\mathbf{1 f}\left(100 \mathrm{MHz}, \mathrm{CDCl}_{3}\right)$
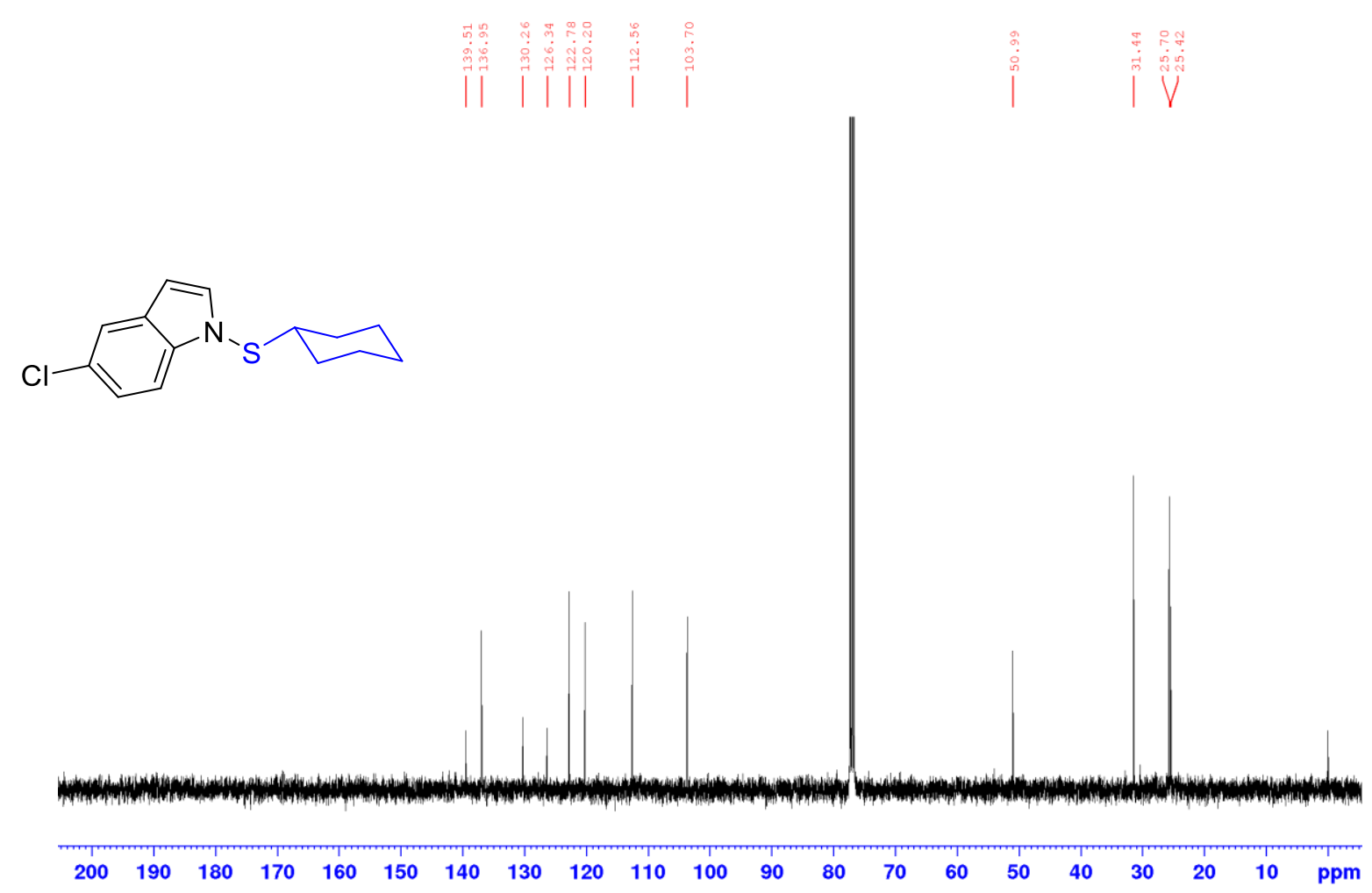
${ }^{1} \mathrm{H}$ NMR of $\mathbf{1 g}\left(400 \mathrm{MHz}, \mathrm{CDCl}_{3}\right)$
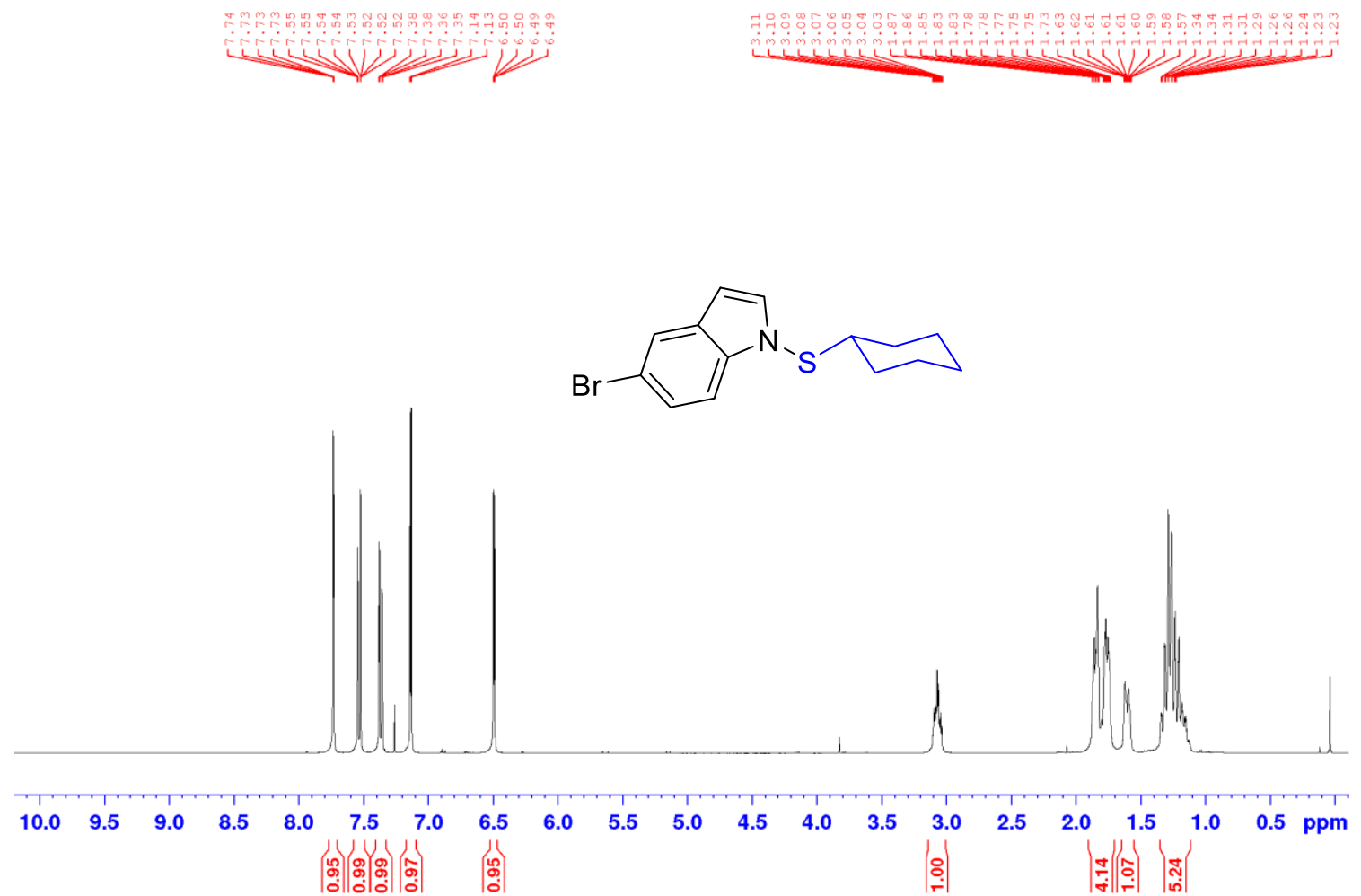

${ }^{13} \mathrm{C}$ NMR of 1 g $\left(100 \mathrm{MHz}, \mathrm{CDCl}_{3}\right)$
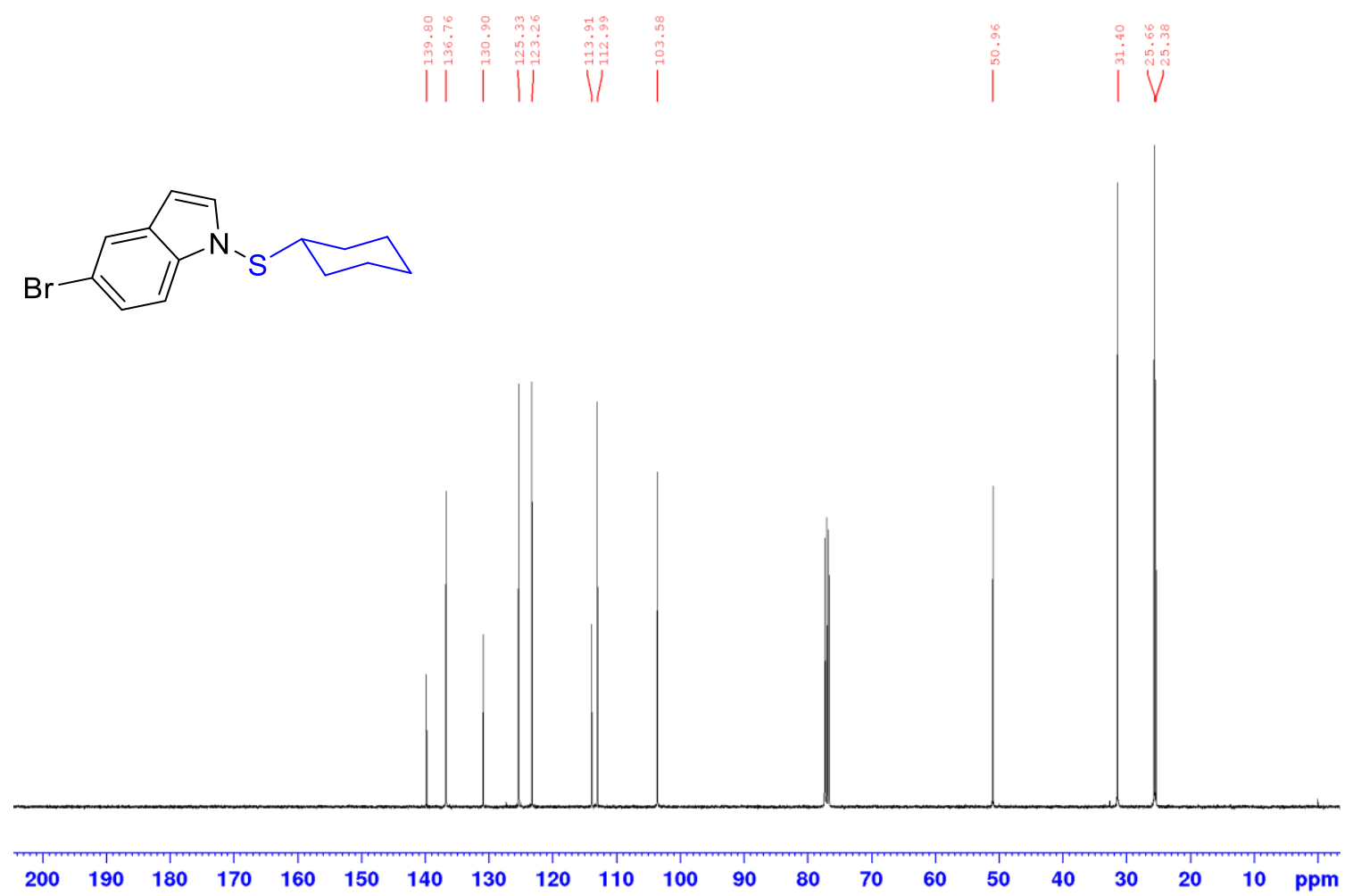
${ }^{1} \mathrm{H}$ NMR of $\mathbf{1 h}\left(400 \mathrm{MHz}, \mathrm{CDCl}_{3}\right)$
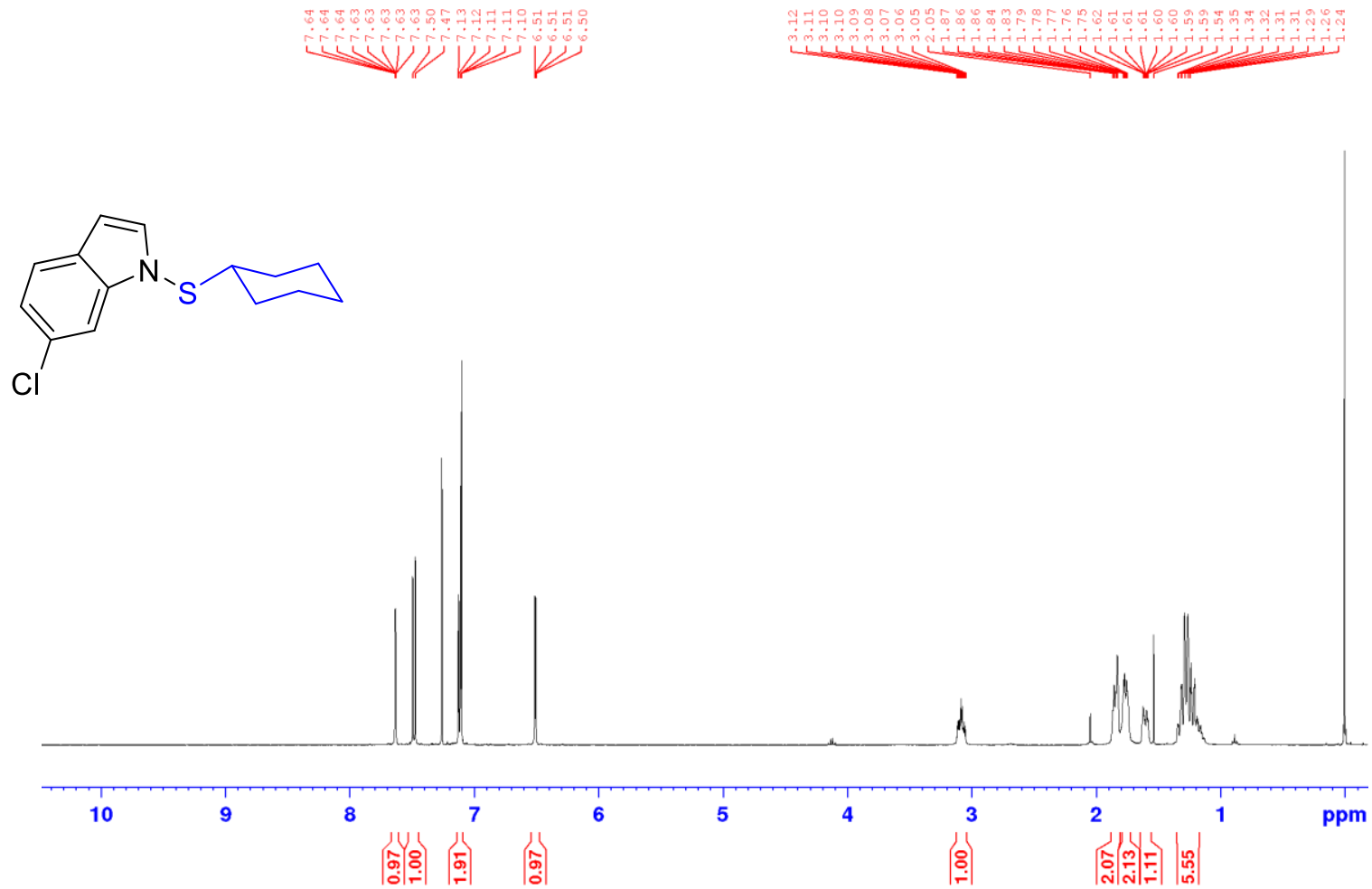

${ }^{13} \mathrm{C}$ NMR of $\mathbf{1 h}\left(100 \mathrm{MHz}, \mathrm{CDCl}_{3}\right)$
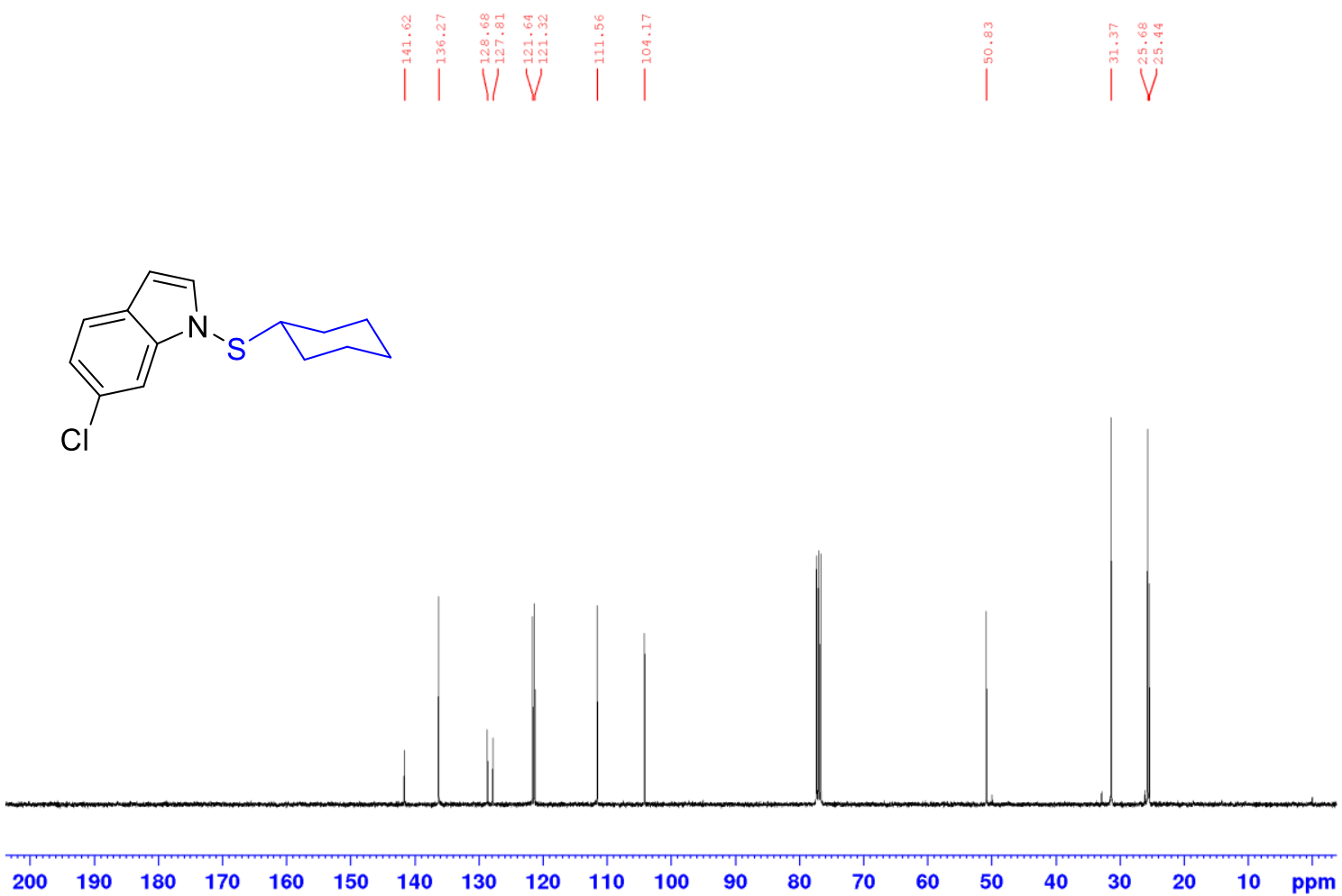
${ }^{1} \mathrm{H}$ NMR of $1 \mathbf{i}\left(400 \mathrm{MHz}, \mathrm{CDCl}_{3}\right)$

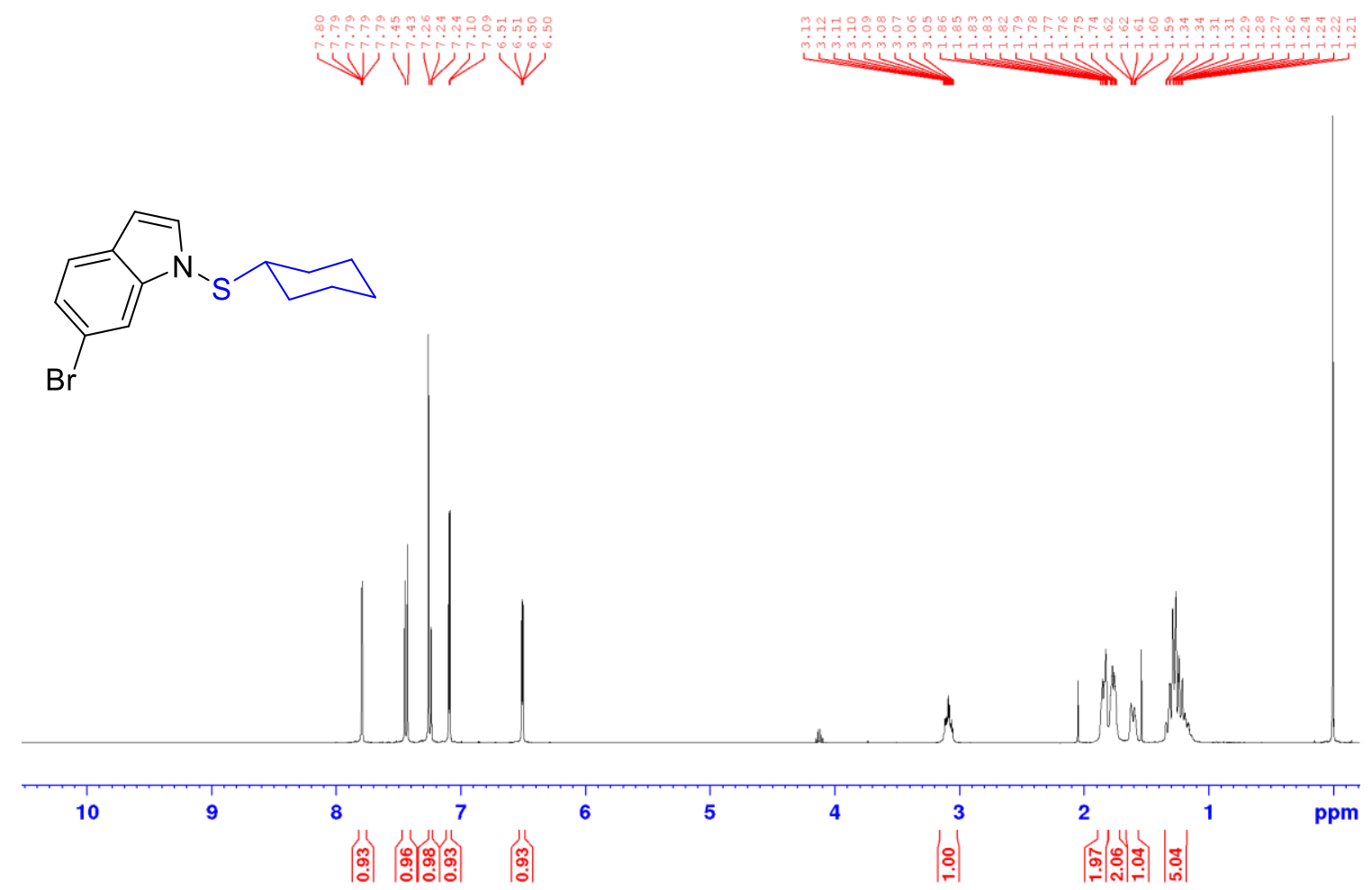

${ }^{13} \mathrm{C}$ NMR of $1 i\left(100 \mathrm{MHz}, \mathrm{CDCl}_{3}\right)$
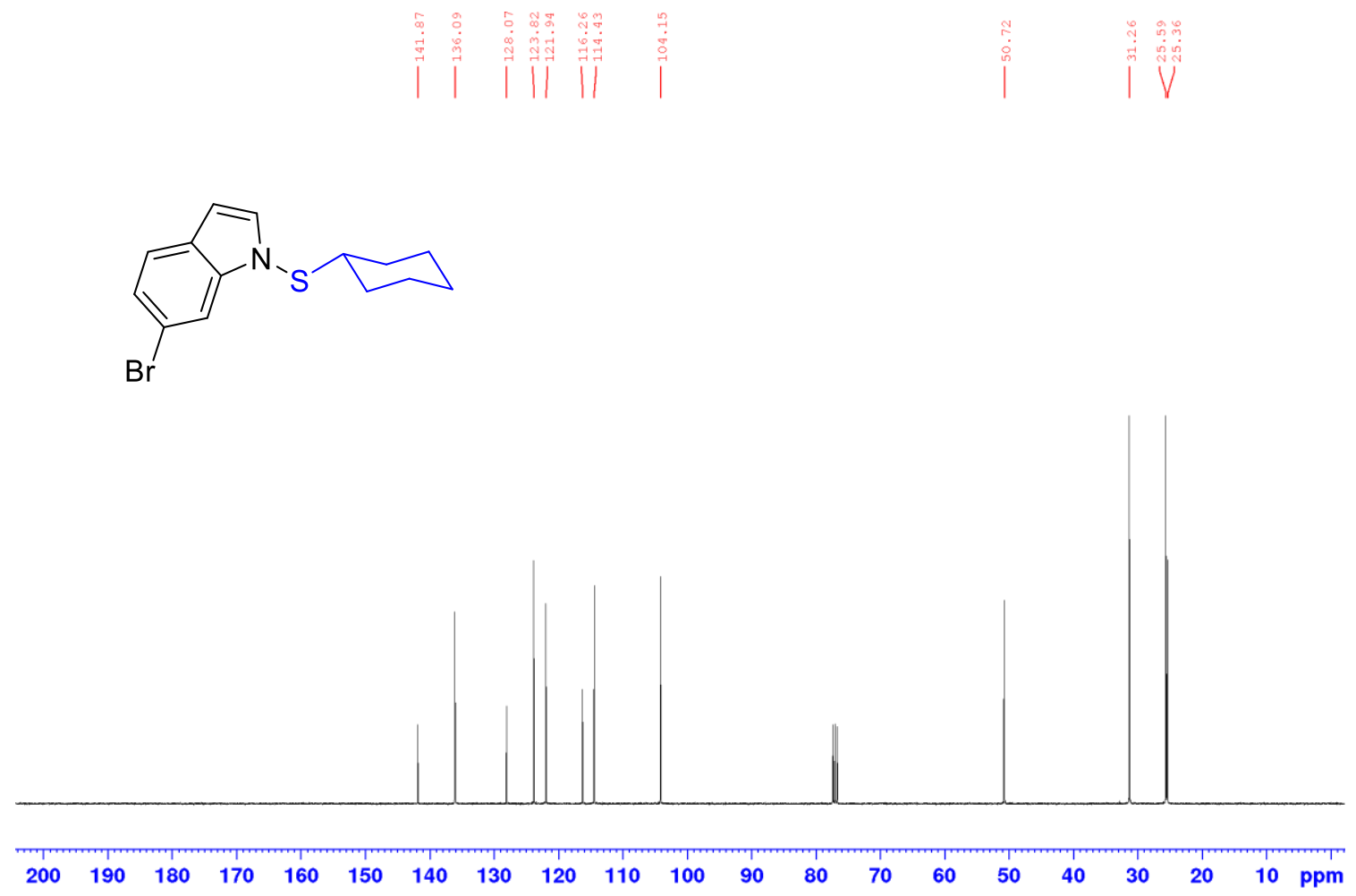
${ }^{1} \mathrm{H}$ NMR of $\mathbf{1 j}\left(400 \mathrm{MHz}, \mathrm{CDCl}_{3}\right.$ )

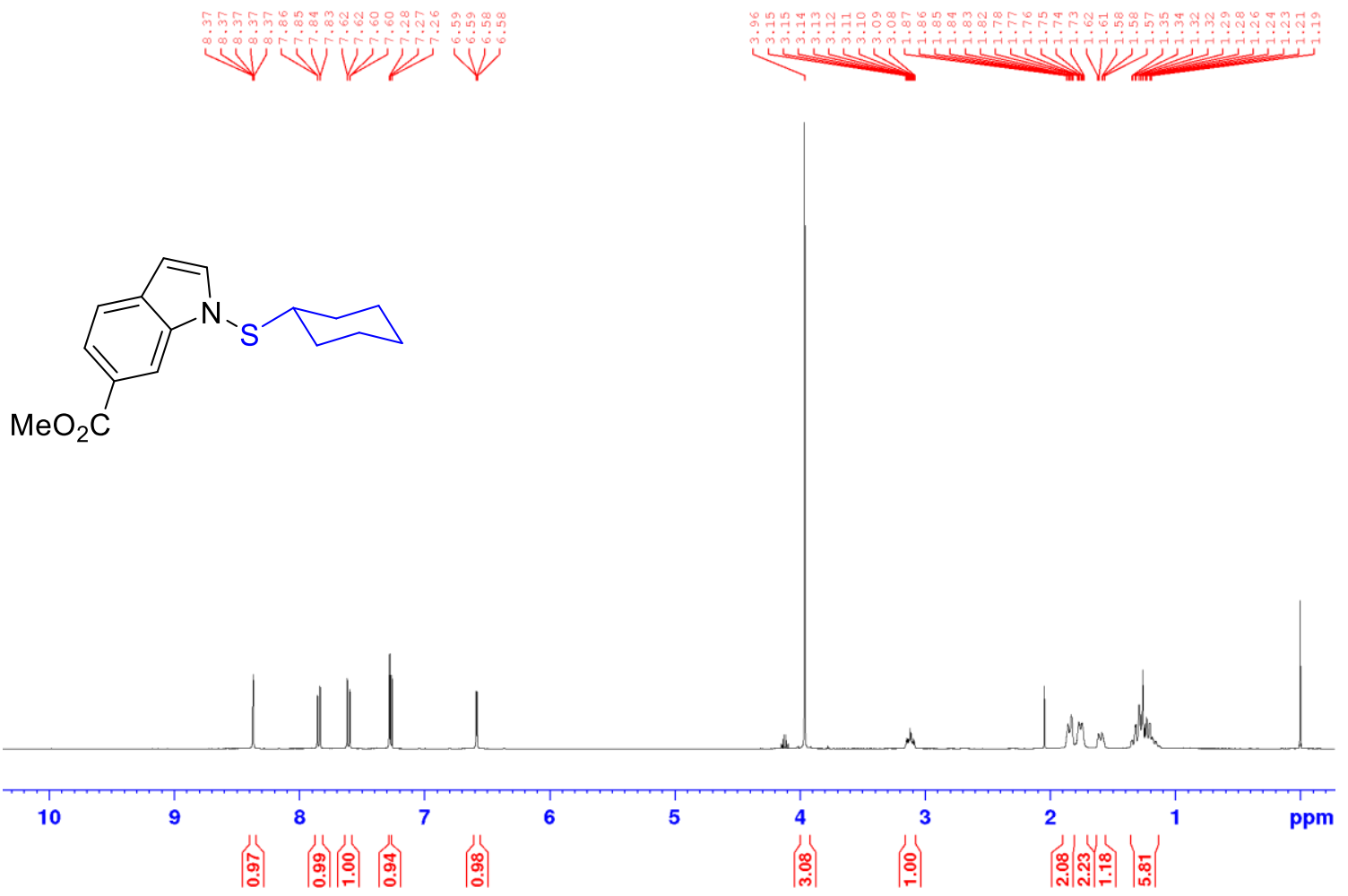

${ }^{13} \mathrm{C}$ NMR of $\mathbf{1 j}\left(100 \mathrm{MHz}, \mathrm{CDCl}_{3}\right)$
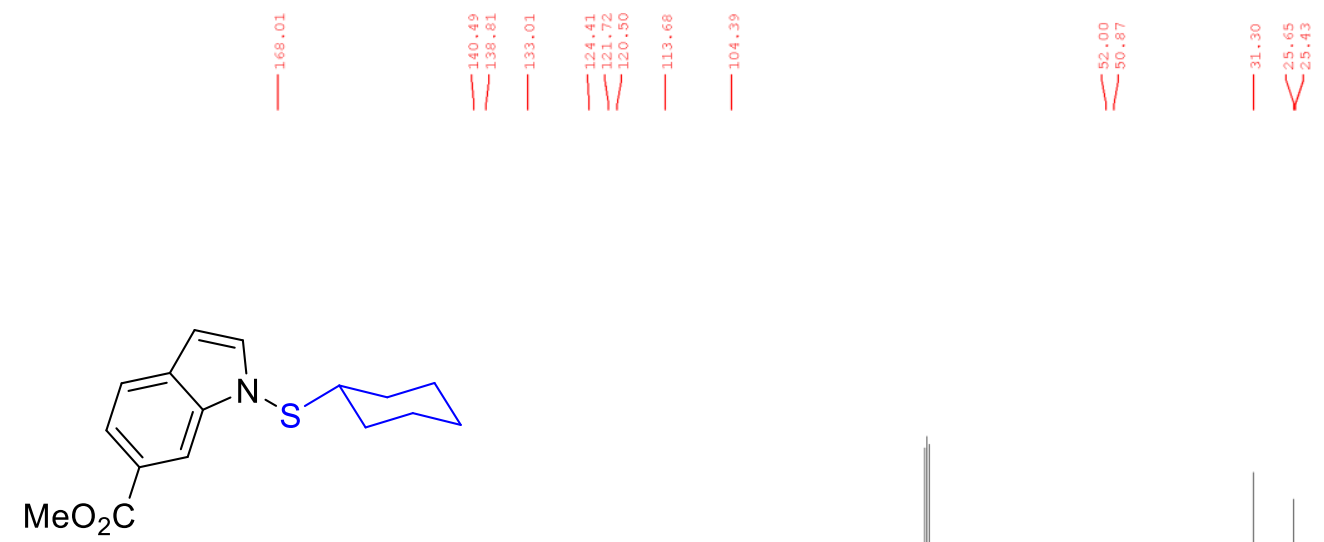
${ }^{1} \mathrm{H}$ NMR of $\mathbf{1 k}\left(400 \mathrm{MHz}, \mathrm{CDCl}_{3}\right)$
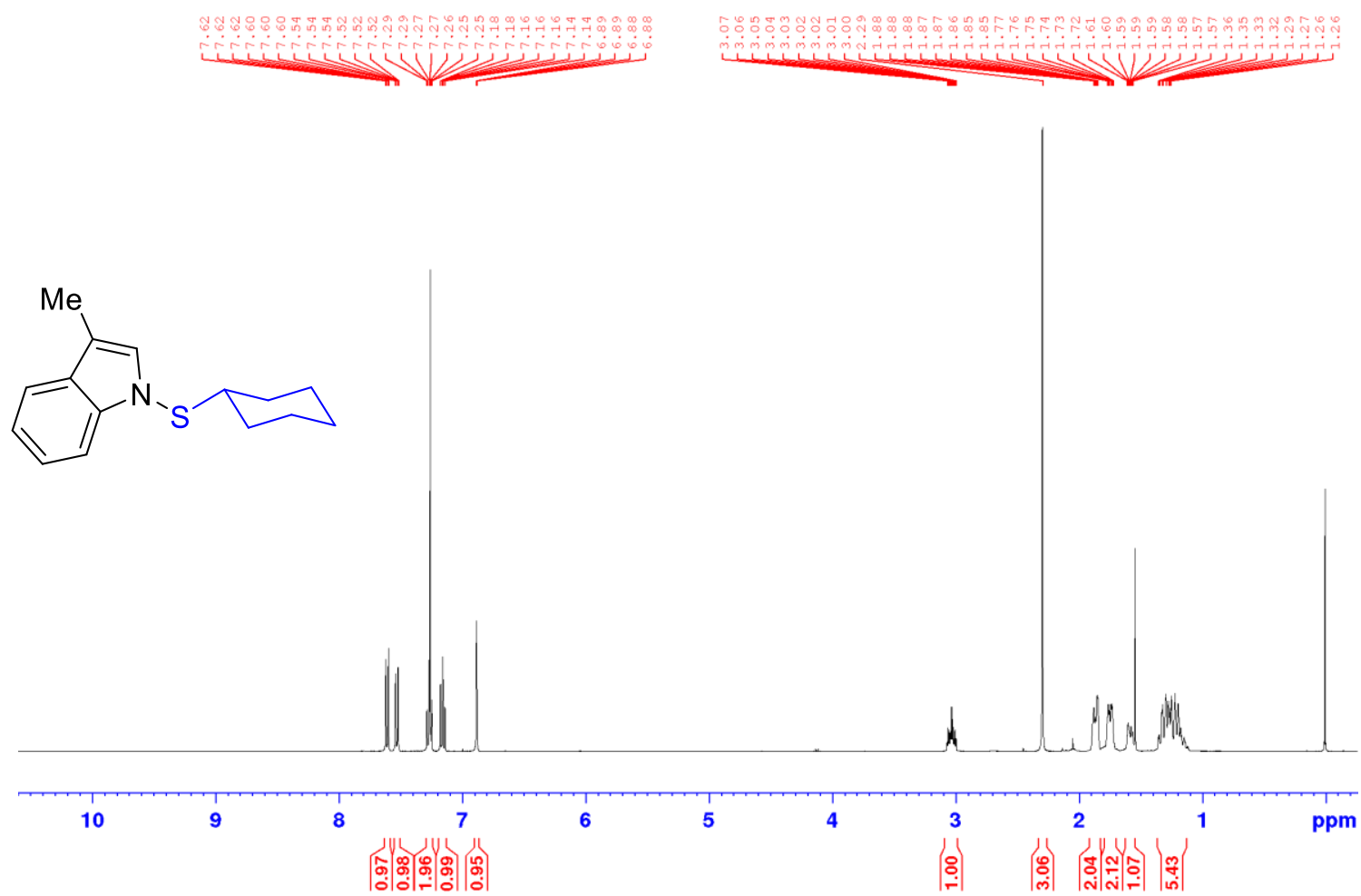

${ }^{13} \mathrm{C} \mathrm{NMR}$ of $\mathbf{1 k}\left(100 \mathrm{MHz}, \mathrm{CDCl}_{3}\right)$<smiles>Cc1cn(SC2CCCC2)c2ccccc12</smiles>

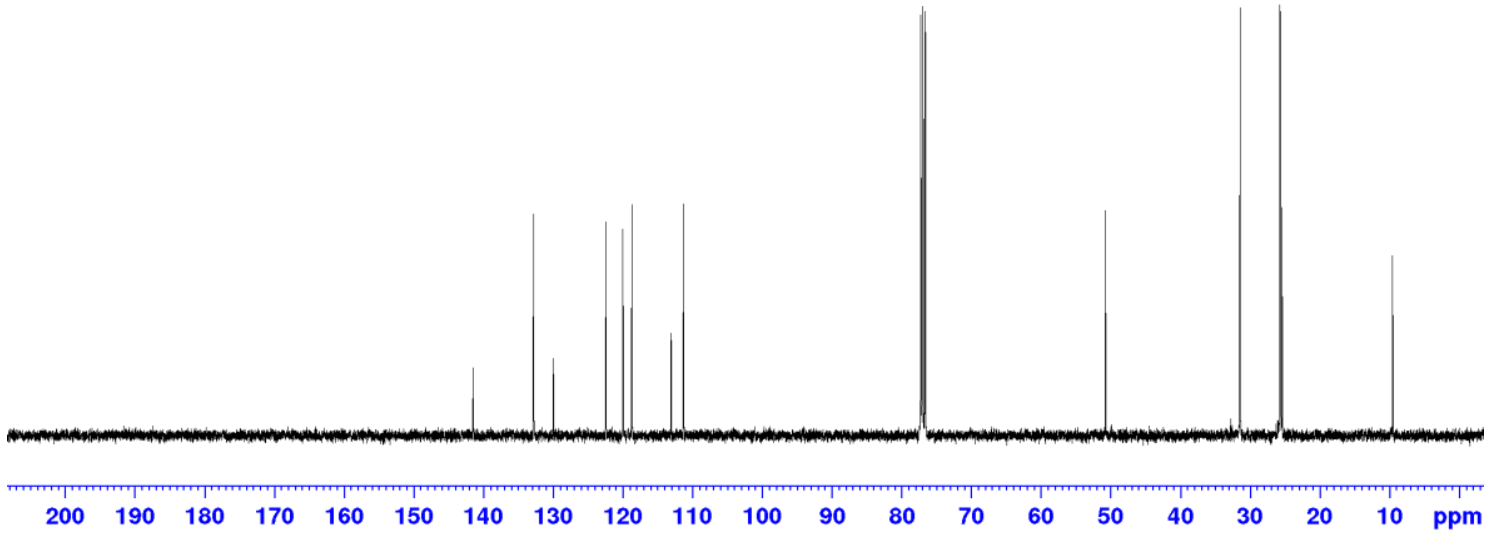


${ }^{1} \mathrm{H}$ NMR of 11 (400 MHz, $\mathrm{CDCl}_{3}$ )

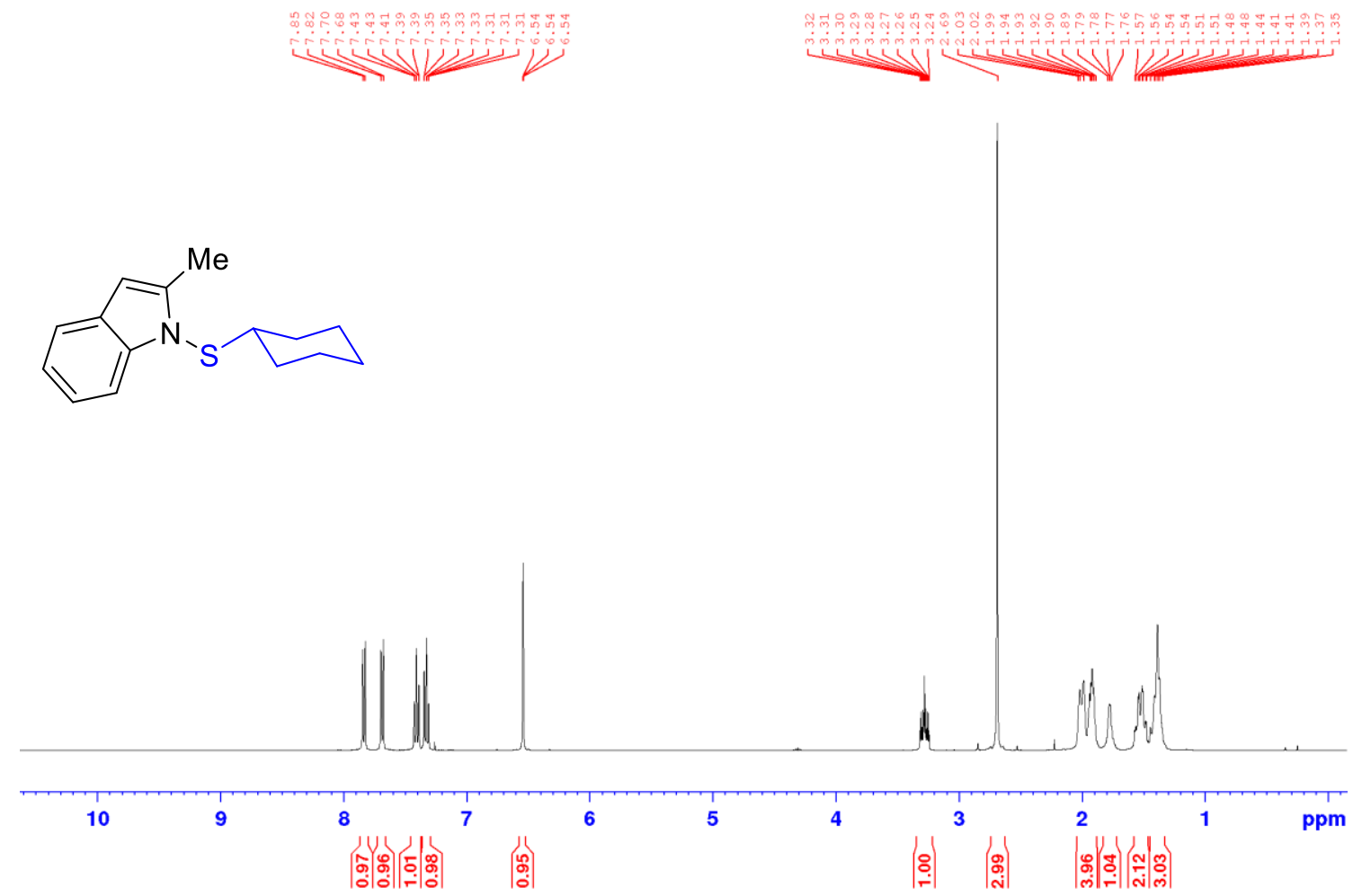

${ }^{13} \mathrm{C}$ NMR of $11\left(100 \mathrm{MHz}, \mathrm{CDCl}_{3}\right)$
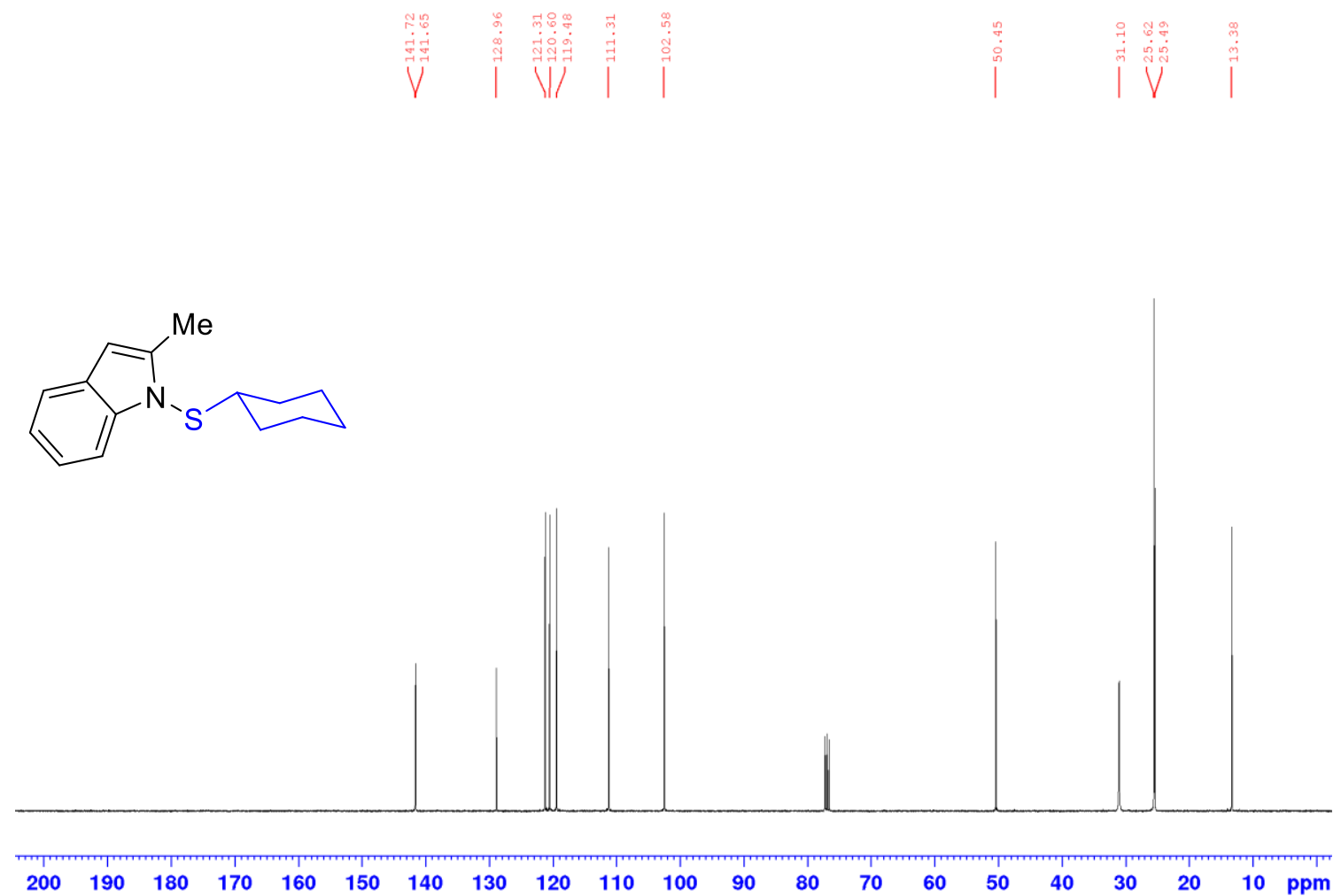
${ }^{1} \mathrm{H}$ NMR of $1 \mathbf{m}\left(400 \mathrm{MHz}, \mathrm{CDCl}_{3}\right)$
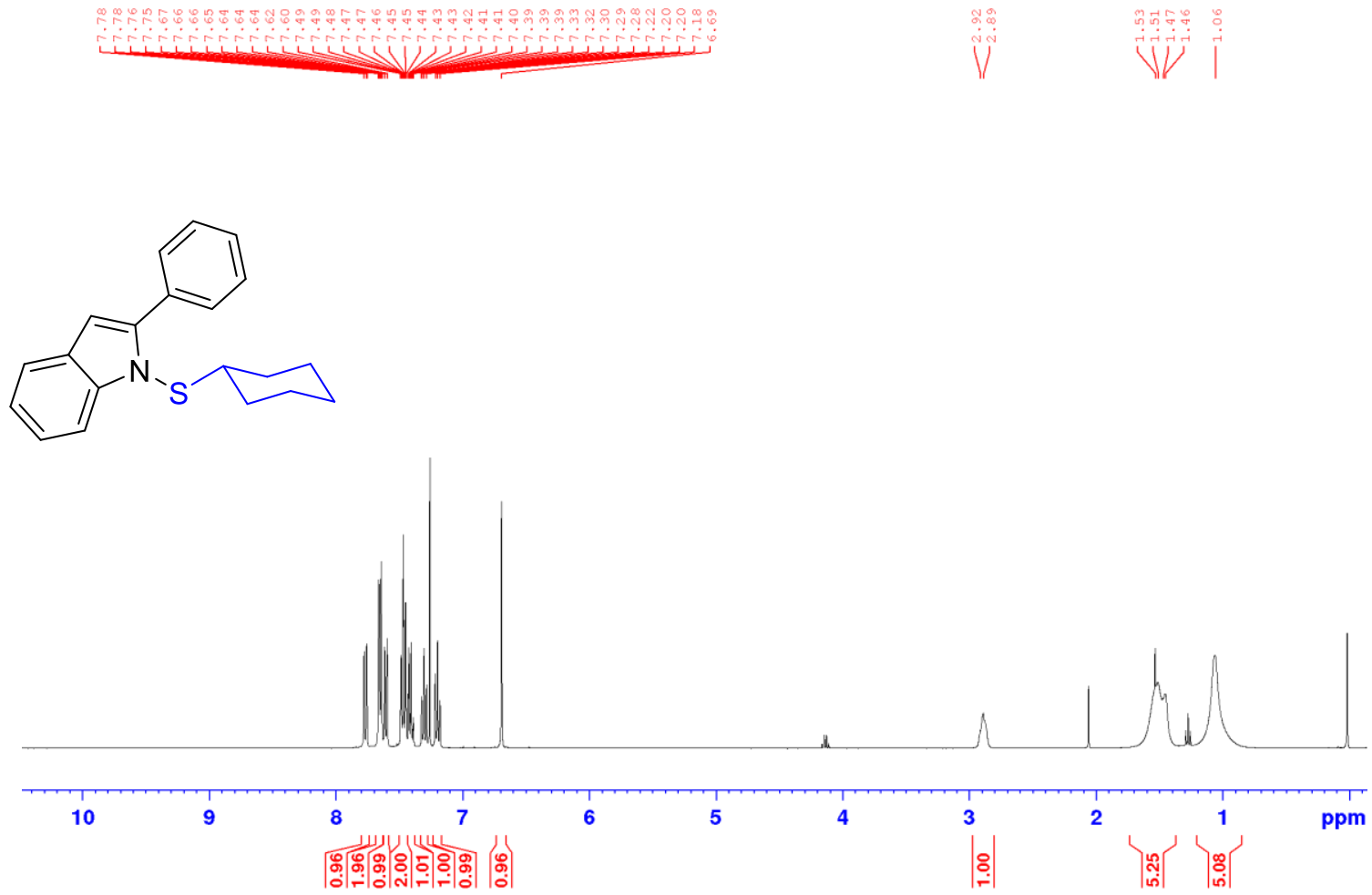

${ }^{13} \mathrm{C}$ NMR of $1 \mathbf{m}\left(100 \mathrm{MHz}, \mathrm{CDCl}_{3}\right)$
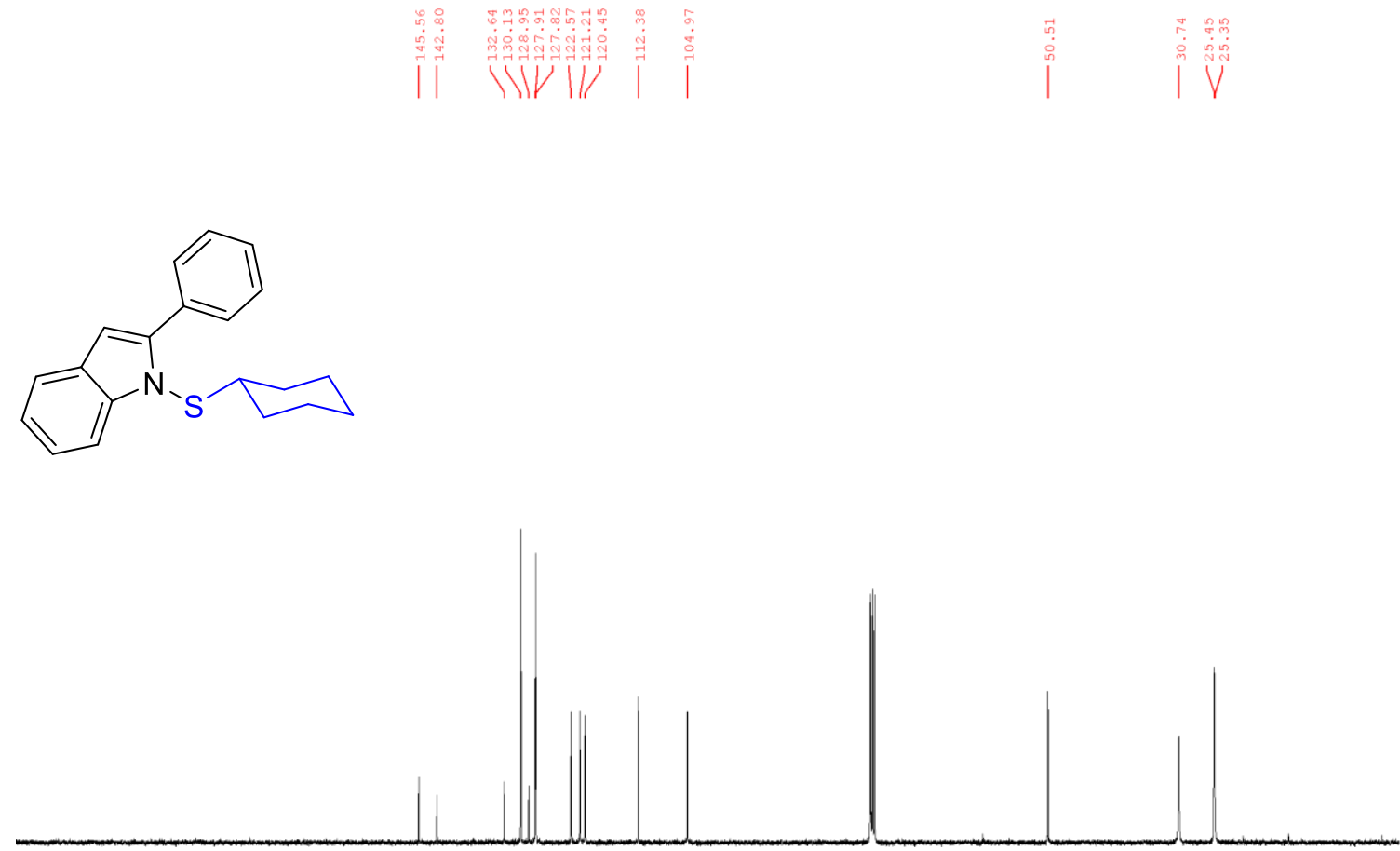

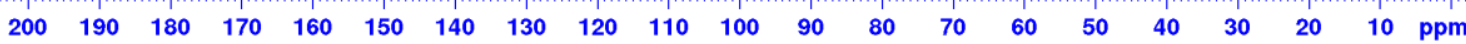


${ }^{1} \mathrm{H}$ NMR of 1 (n $\left(400 \mathrm{MHz}, \mathrm{CDCl}_{3}\right)$

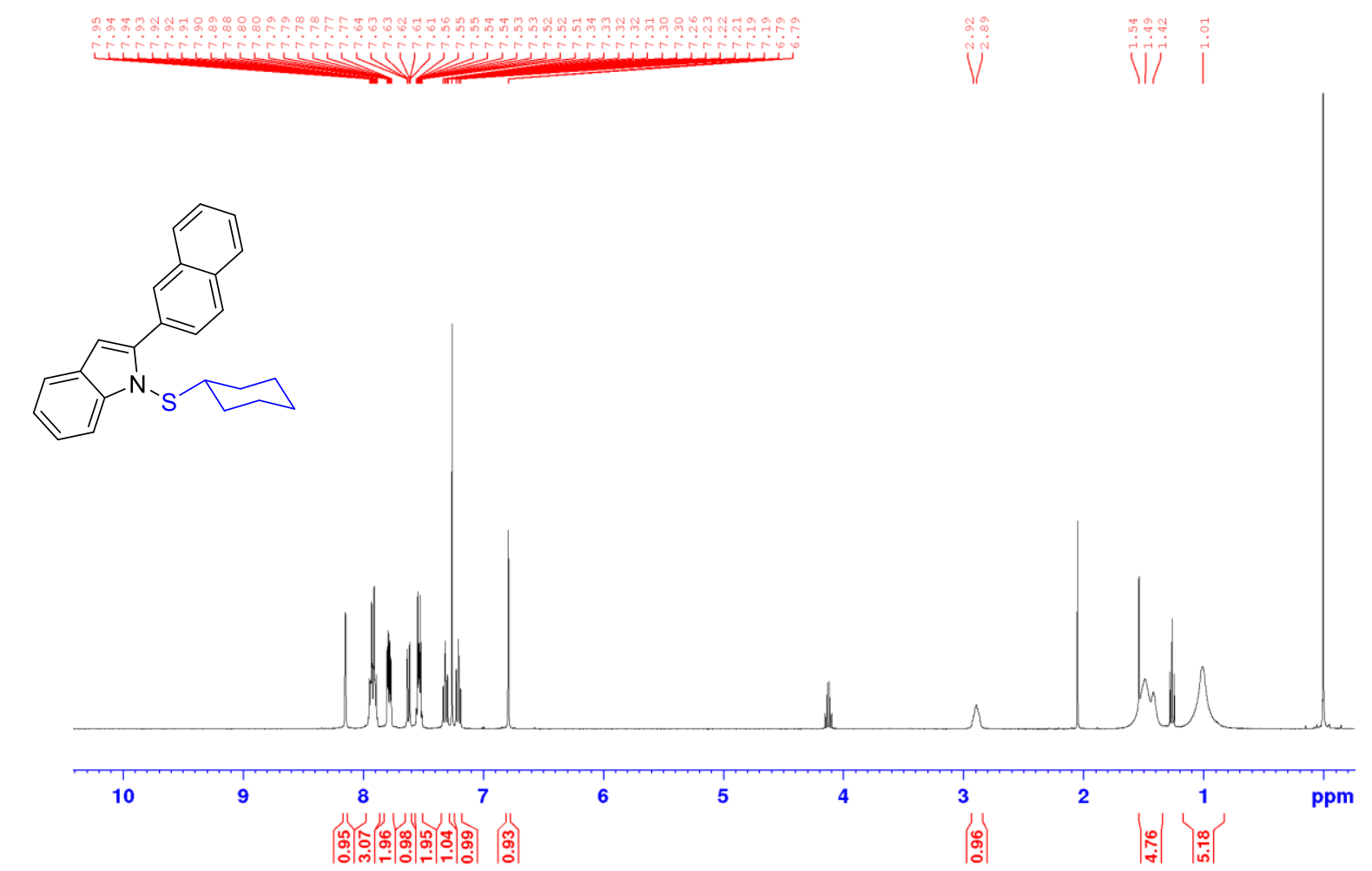

${ }^{13} \mathrm{C}$ NMR of 1 n $\left(100 \mathrm{MHz}, \mathrm{CDCl}_{3}\right)$

|1|
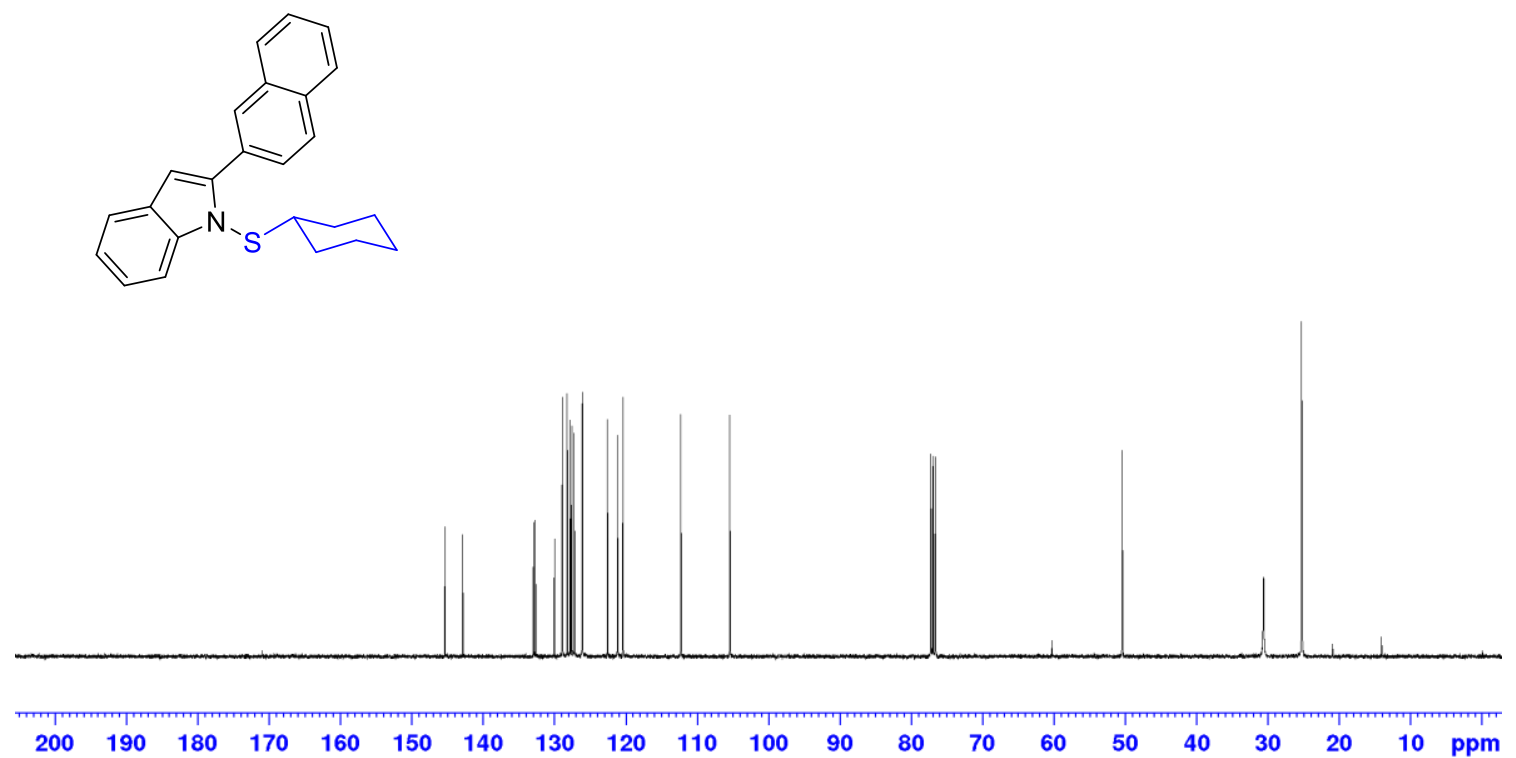
${ }^{1} \mathrm{H}$ NMR of $10\left(400 \mathrm{MHz}, \mathrm{CDCl}_{3}\right)$

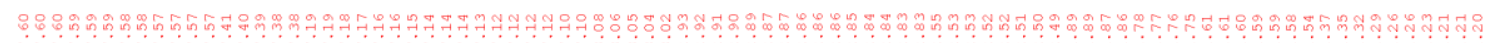

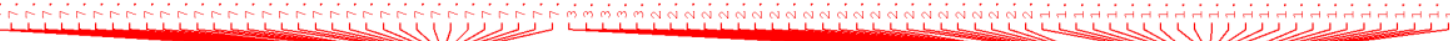<smiles>c1ccc2c(c1)c1c(n2SC2CCCCC2)CCC1</smiles>
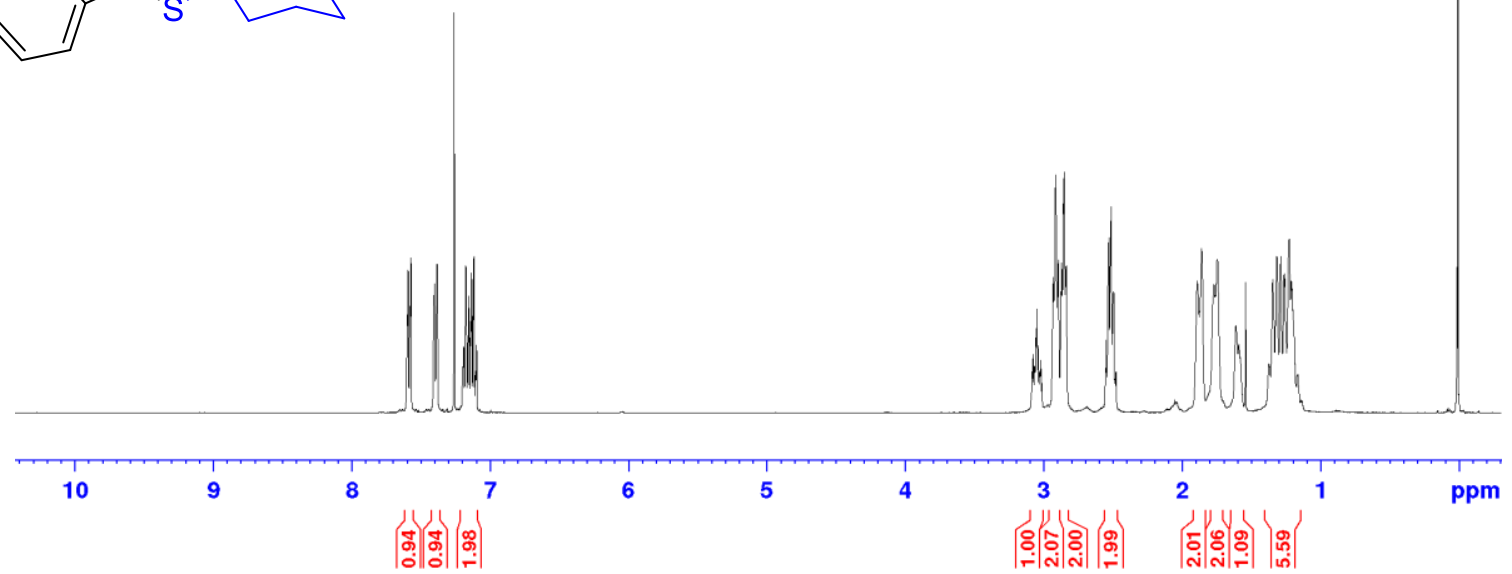

${ }^{13} \mathrm{C} \mathrm{NMR}$ of $10\left(100 \mathrm{MHz}, \mathrm{CDCl}_{3}\right)$

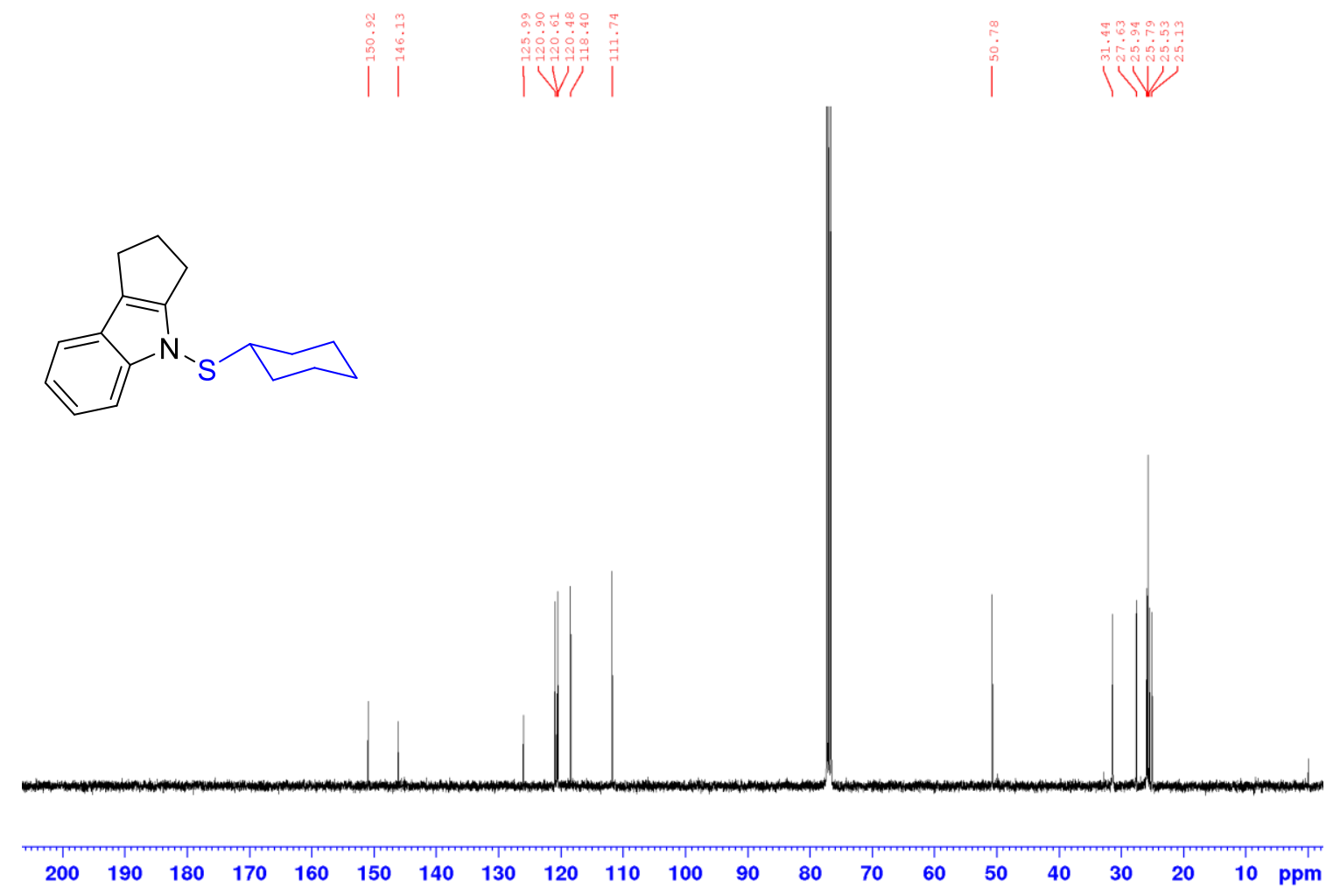


${ }^{1} \mathrm{H}$ NMR of $\mathbf{1 p}\left(400 \mathrm{MHz}, \mathrm{CDCl}_{3}\right.$ )
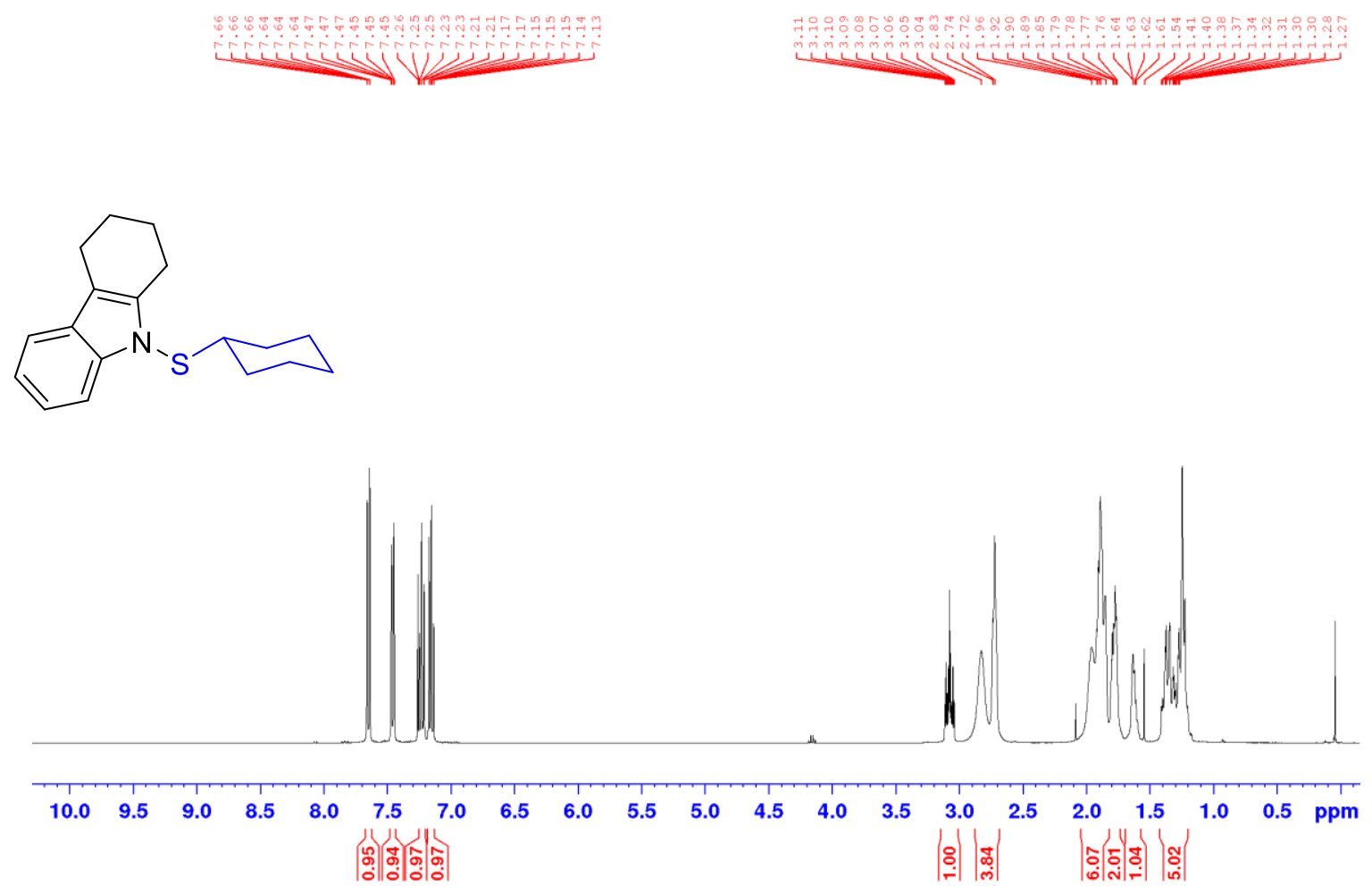

${ }^{13} \mathrm{C} \mathrm{NMR}$ of $1 p\left(100 \mathrm{MHz}, \mathrm{CDCl}_{3}\right)$
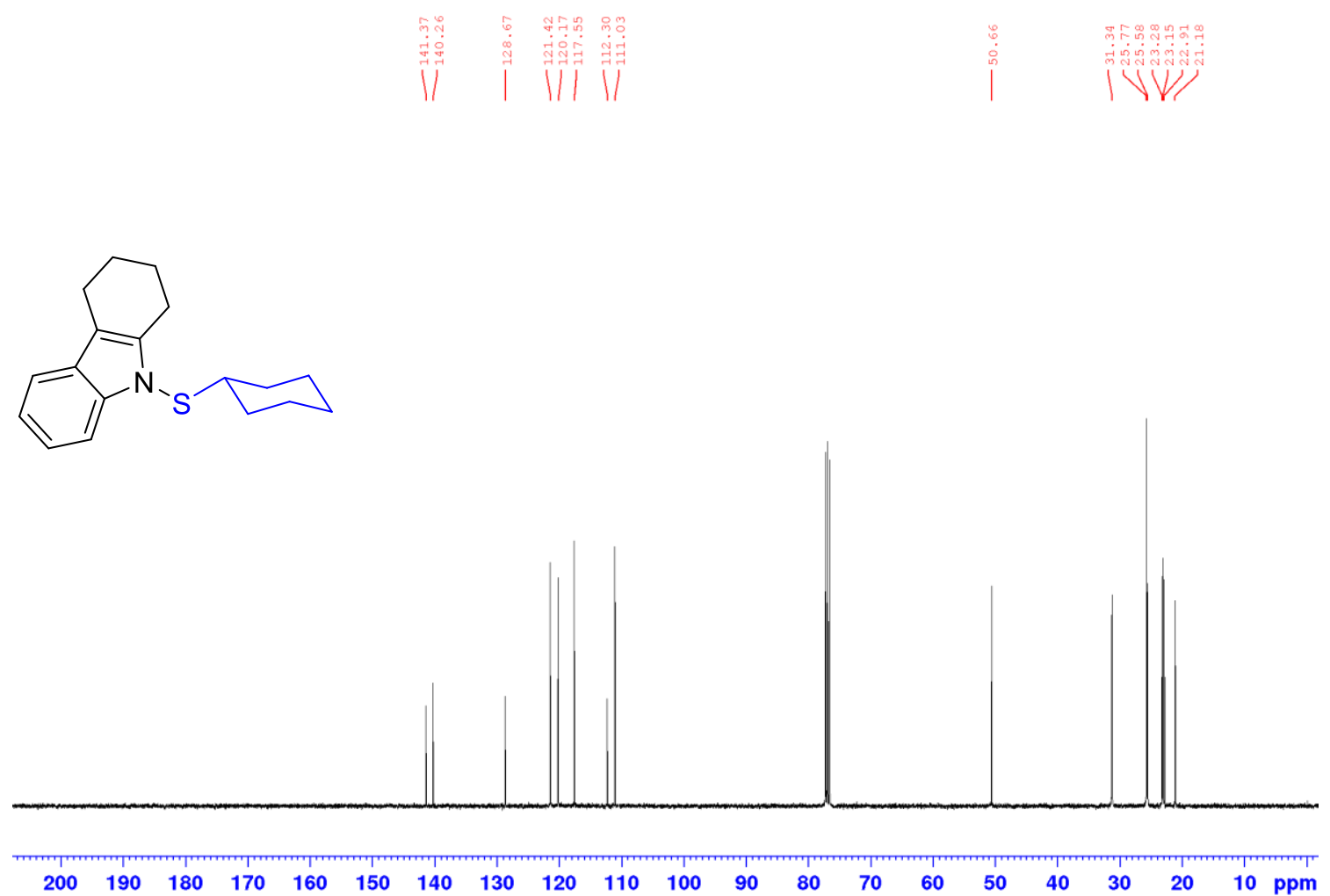
${ }^{1} \mathrm{H}$ NMR of 1q (400 MHz, $\left.\mathrm{CDCl}_{3}\right)$
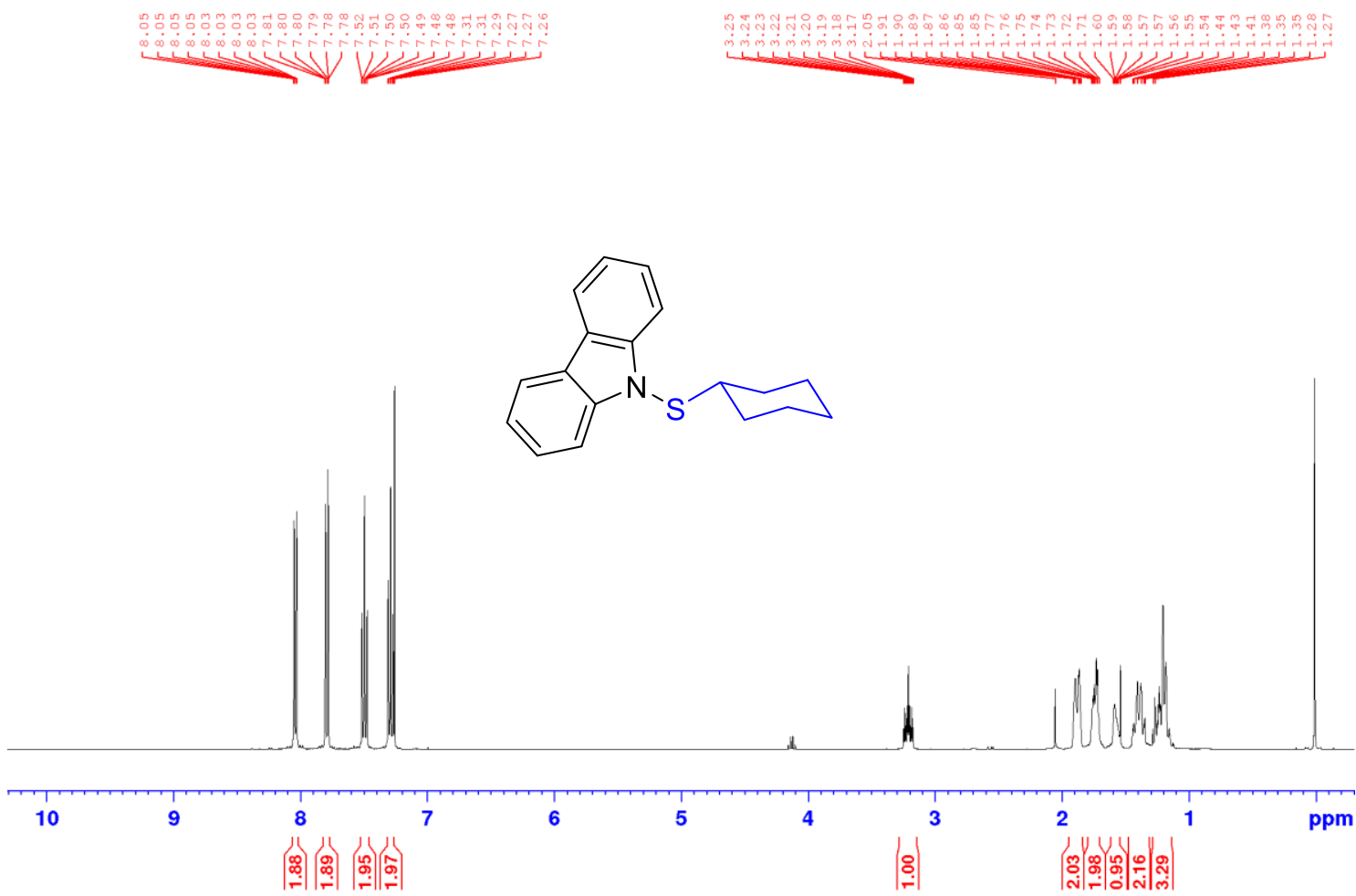

${ }^{13} \mathrm{C} \mathrm{NMR}$ of $1 \mathbf{q}\left(100 \mathrm{MHz}, \mathrm{CDCl}_{3}\right)$
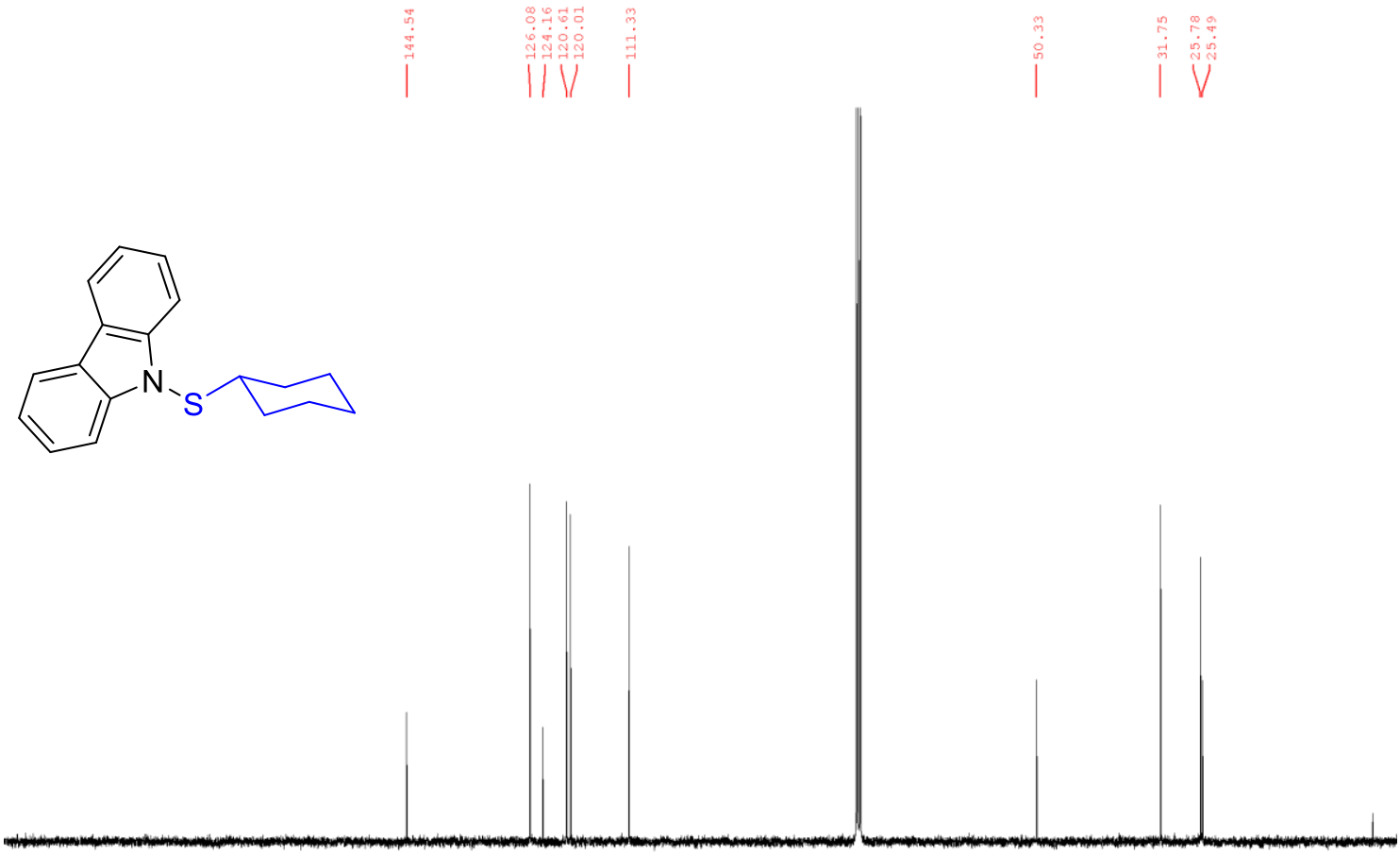

$\begin{array}{llllllllllllllllllllll}200 & 190 & 180 & 170 & 160 & 150 & 140 & 130 & 120 & 110 & 100 & 90 & 80 & 70 & 60 & 50 & 40 & 30 & 20 & 10 & \text { ppm }\end{array}$ 
${ }^{1} \mathrm{H} \mathrm{NMR}$ of $\mathbf{3 b a}\left(400 \mathrm{MHz}, \mathrm{CDCl}_{3}\right)$

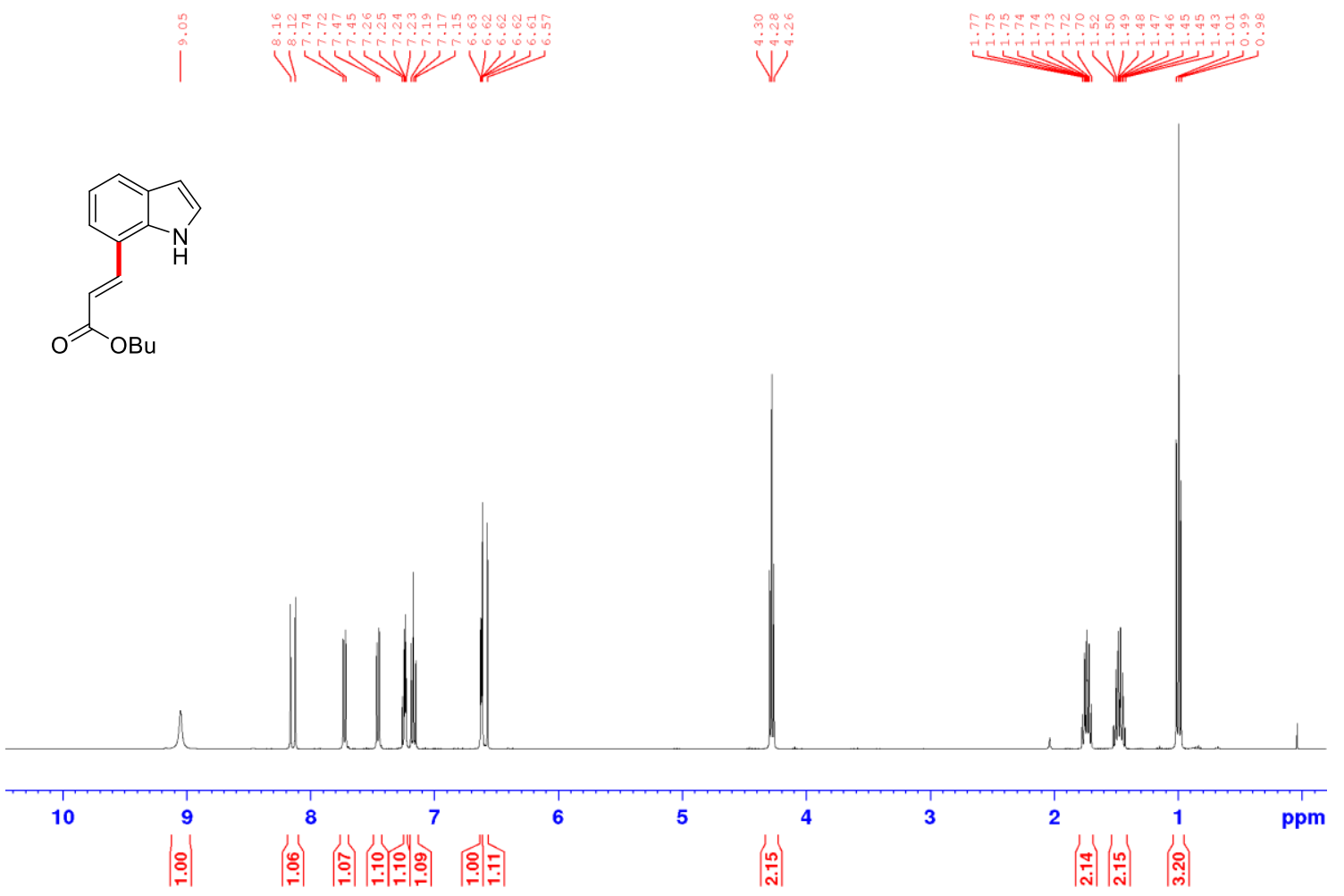

${ }^{13} \mathrm{C}$ NMR of $\mathbf{3 b a}\left(100 \mathrm{MHz}, \mathrm{CDCl}_{3}\right.$

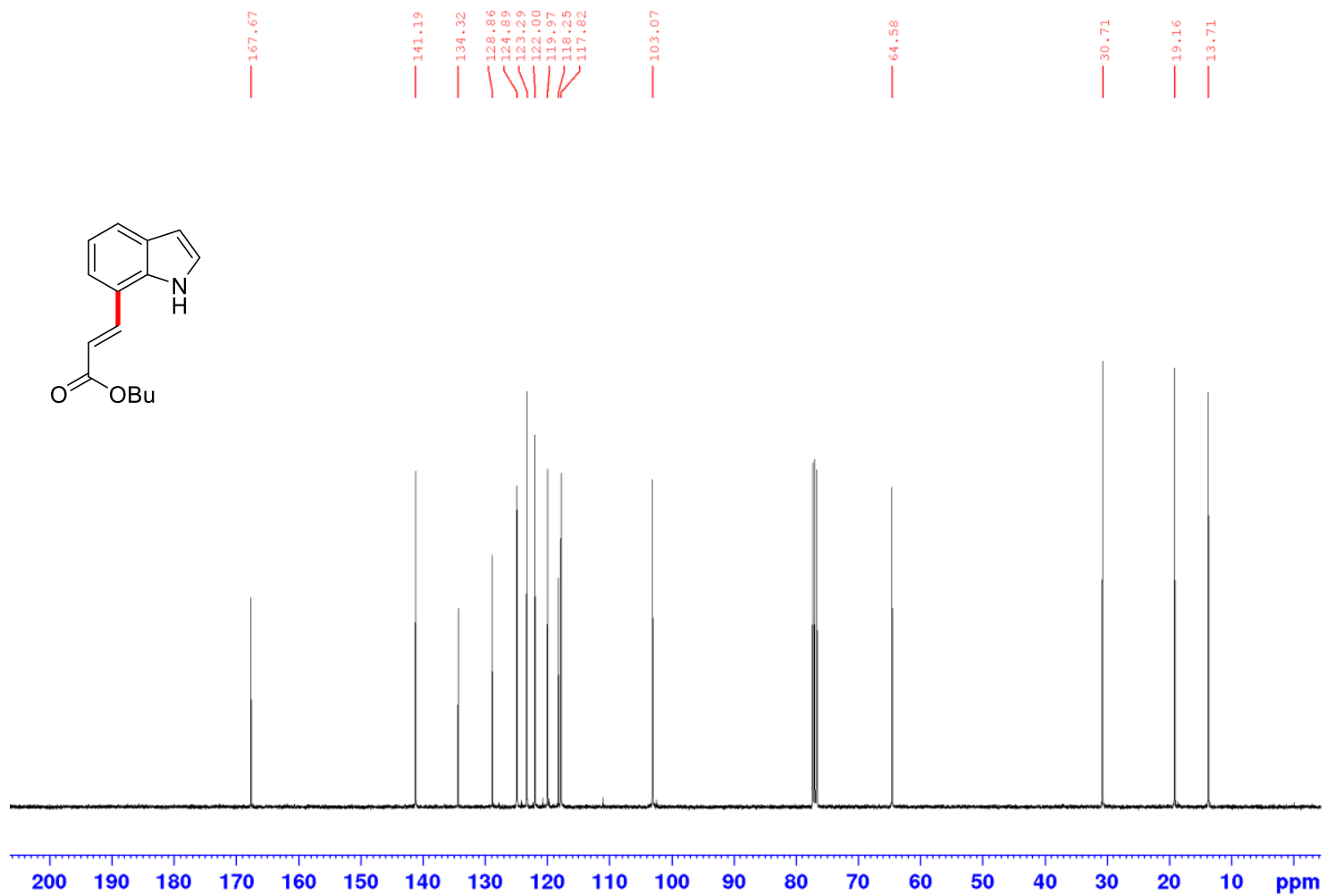


${ }^{1} \mathrm{H}$ NMR of $\mathbf{3 b b}\left(400 \mathrm{MHz}, \mathrm{CDCl}_{3}\right)$

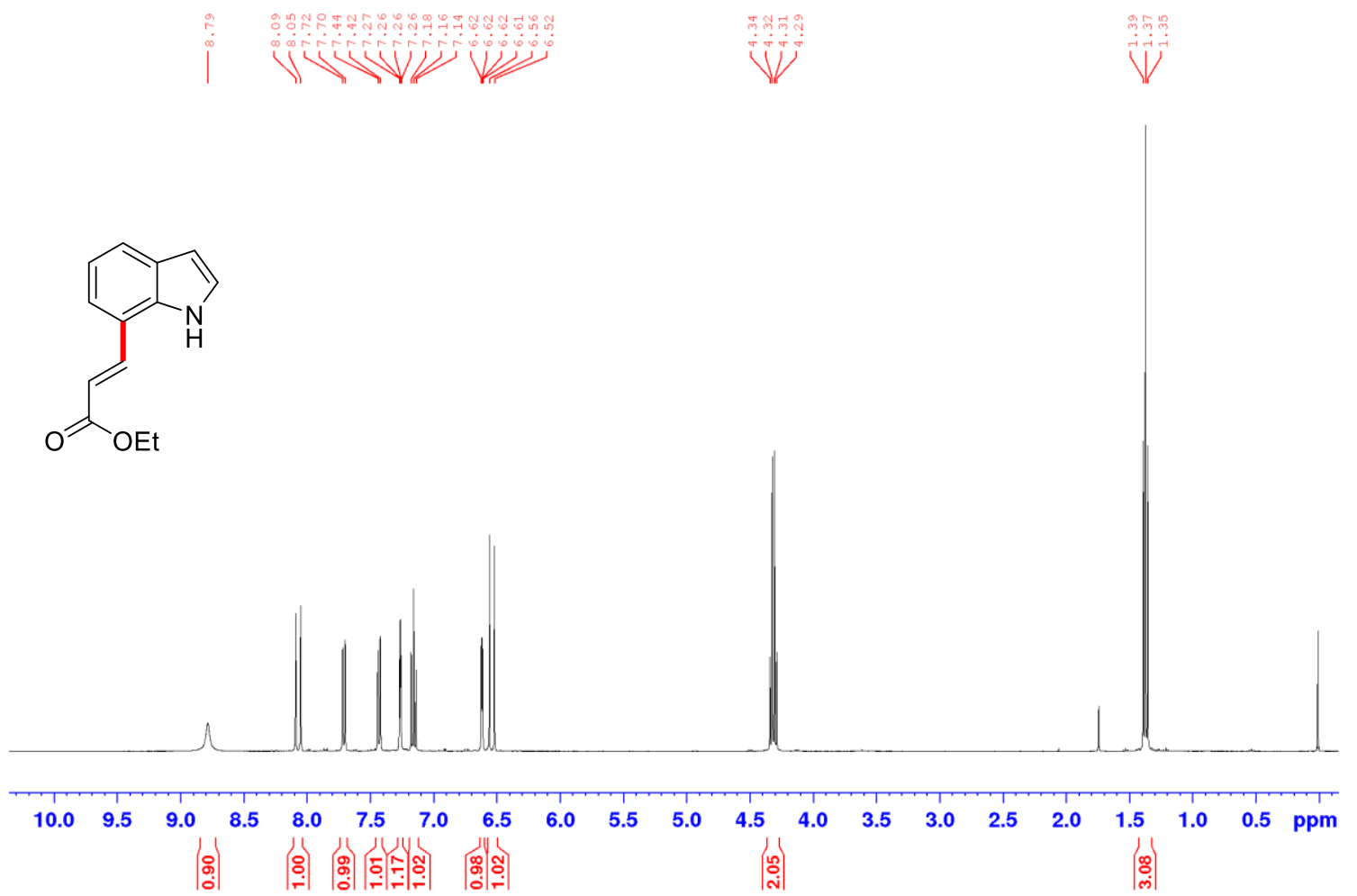

${ }^{13} \mathrm{C}$ NMR of $\mathbf{3 b b}\left(100 \mathrm{MHz}, \mathrm{CDCl}_{3}\right.$

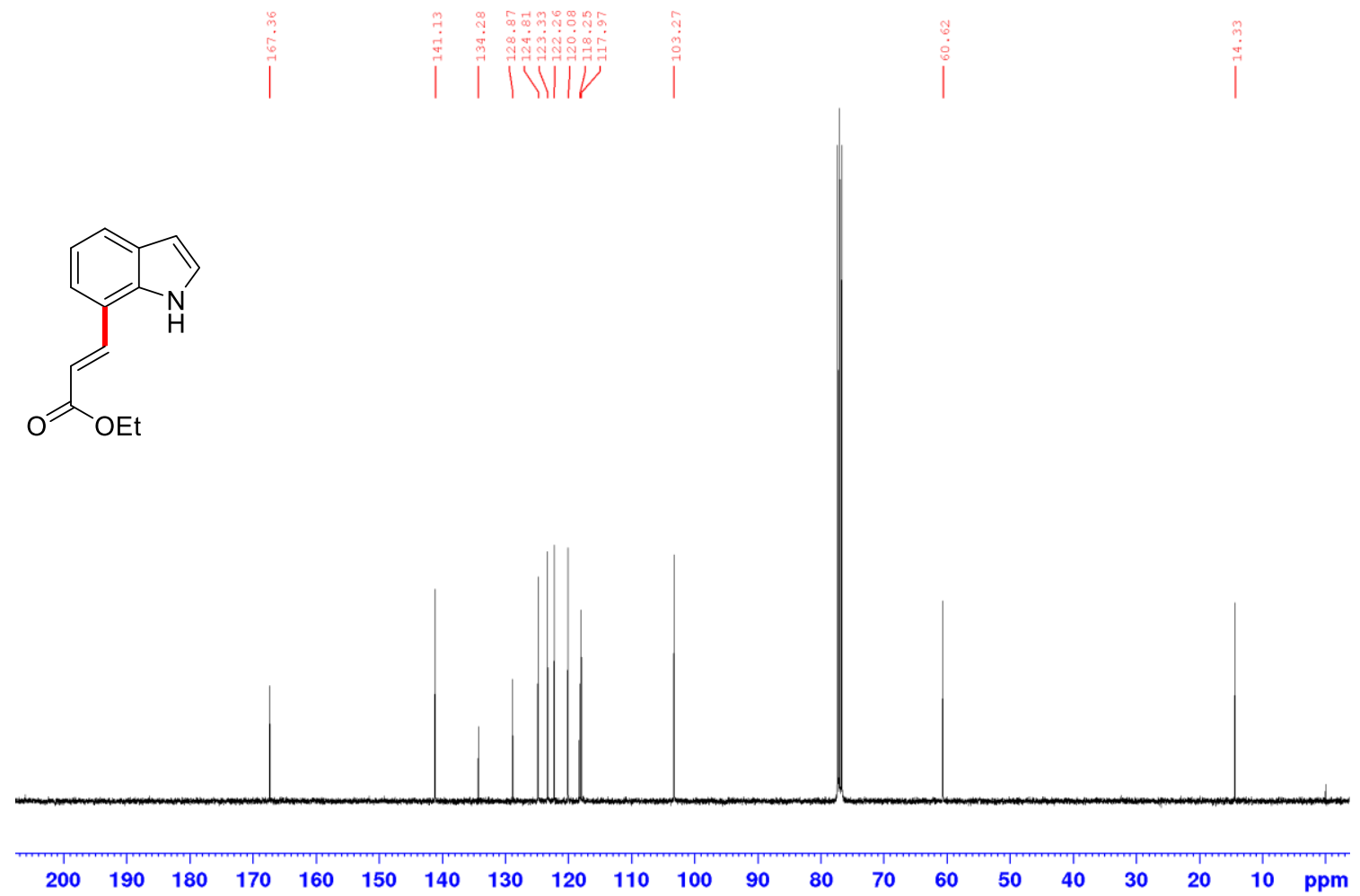


${ }^{1} \mathrm{H}$ NMR of $3 \mathbf{b c}\left(400 \mathrm{MHz}, \mathrm{CDCl}_{3}\right)$

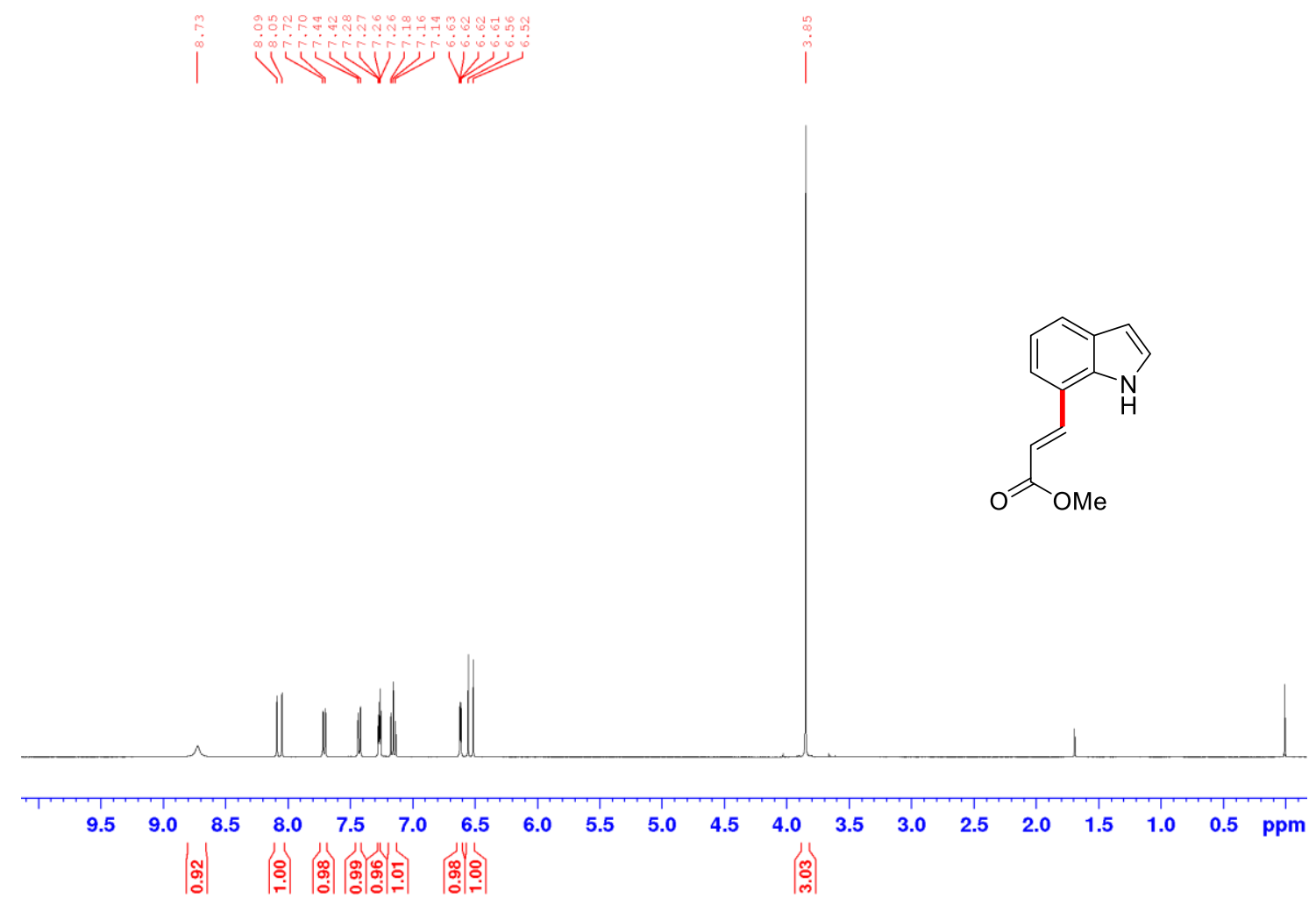

${ }^{13} \mathrm{C}$ NMR of 3 bc $\left(100 \mathrm{MHz}, \mathrm{CDCl}_{3}\right)$
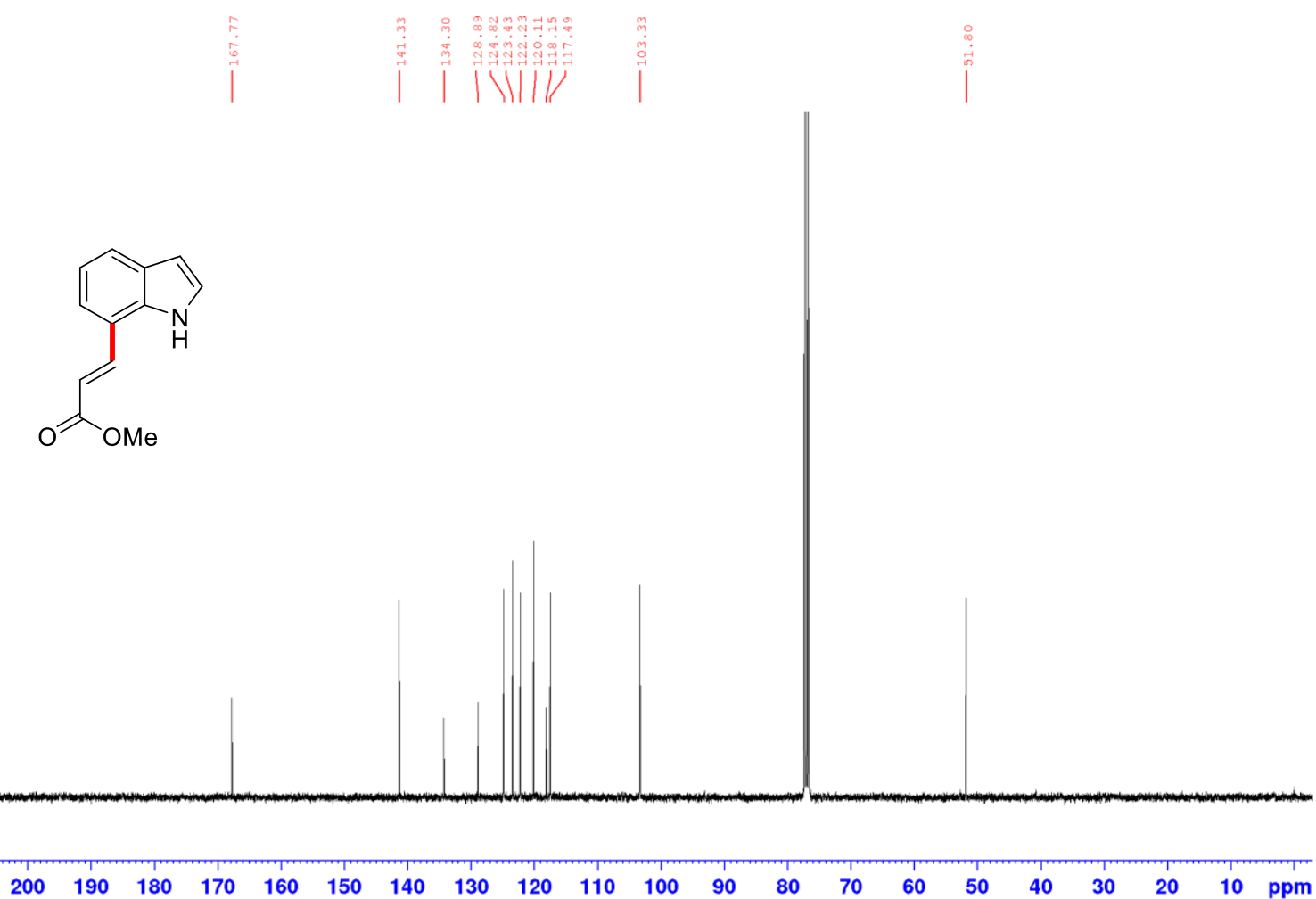
${ }^{1} \mathrm{H}$ NMR of $3 \mathbf{b d}\left(400 \mathrm{MHz}, \mathrm{CDCl}_{3}\right)$

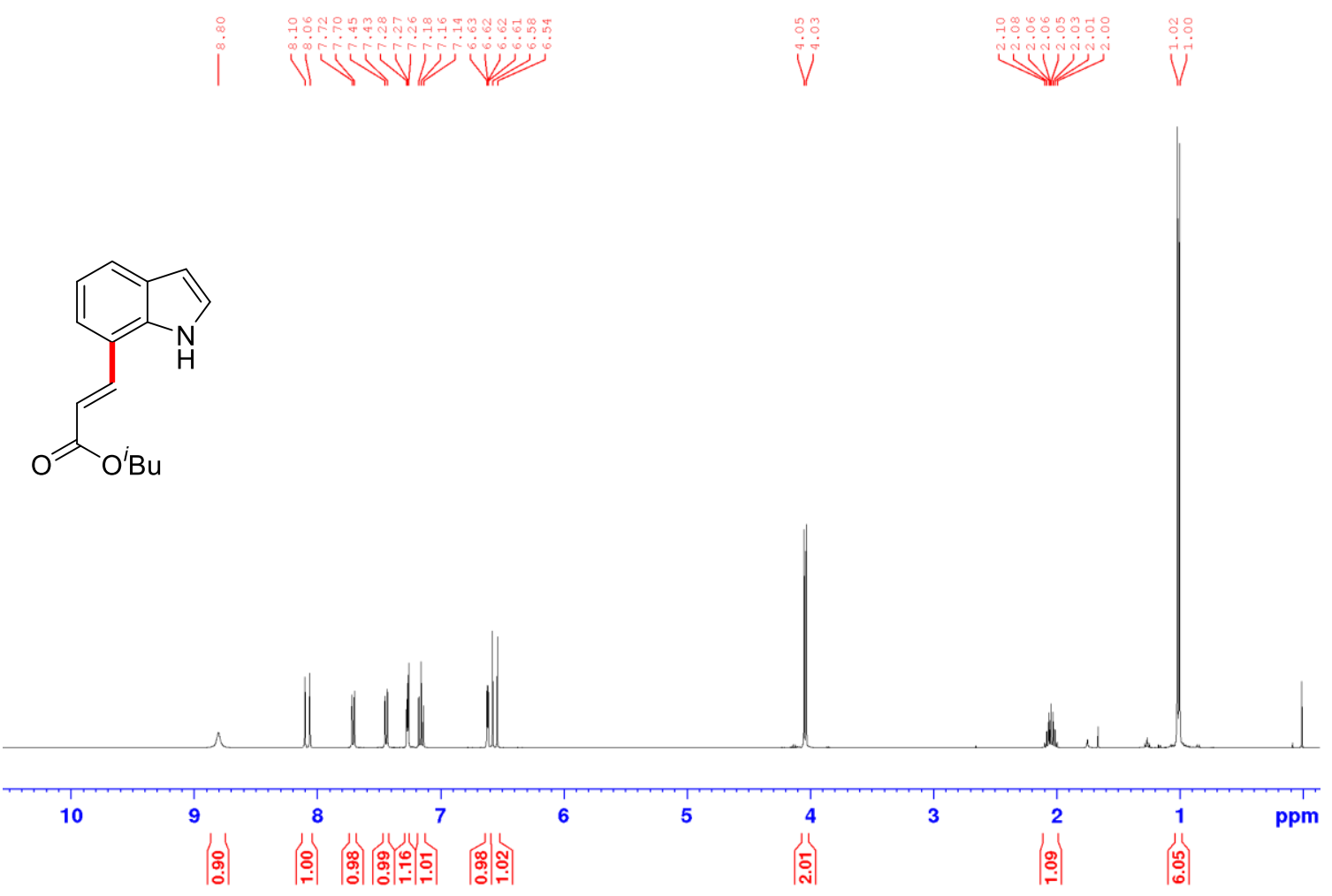

${ }^{13} \mathrm{C}$ NMR of 3 bd $\left(100 \mathrm{MHz}, \mathrm{CDCl}_{3}\right)$<smiles>CCCCOC(=O)/C=C/c1cccc2cc[nH]c12</smiles> 
${ }^{1} \mathrm{H}$ NMR of 3be $\left(400 \mathrm{MHz}, \mathrm{CDCl}_{3}\right)$

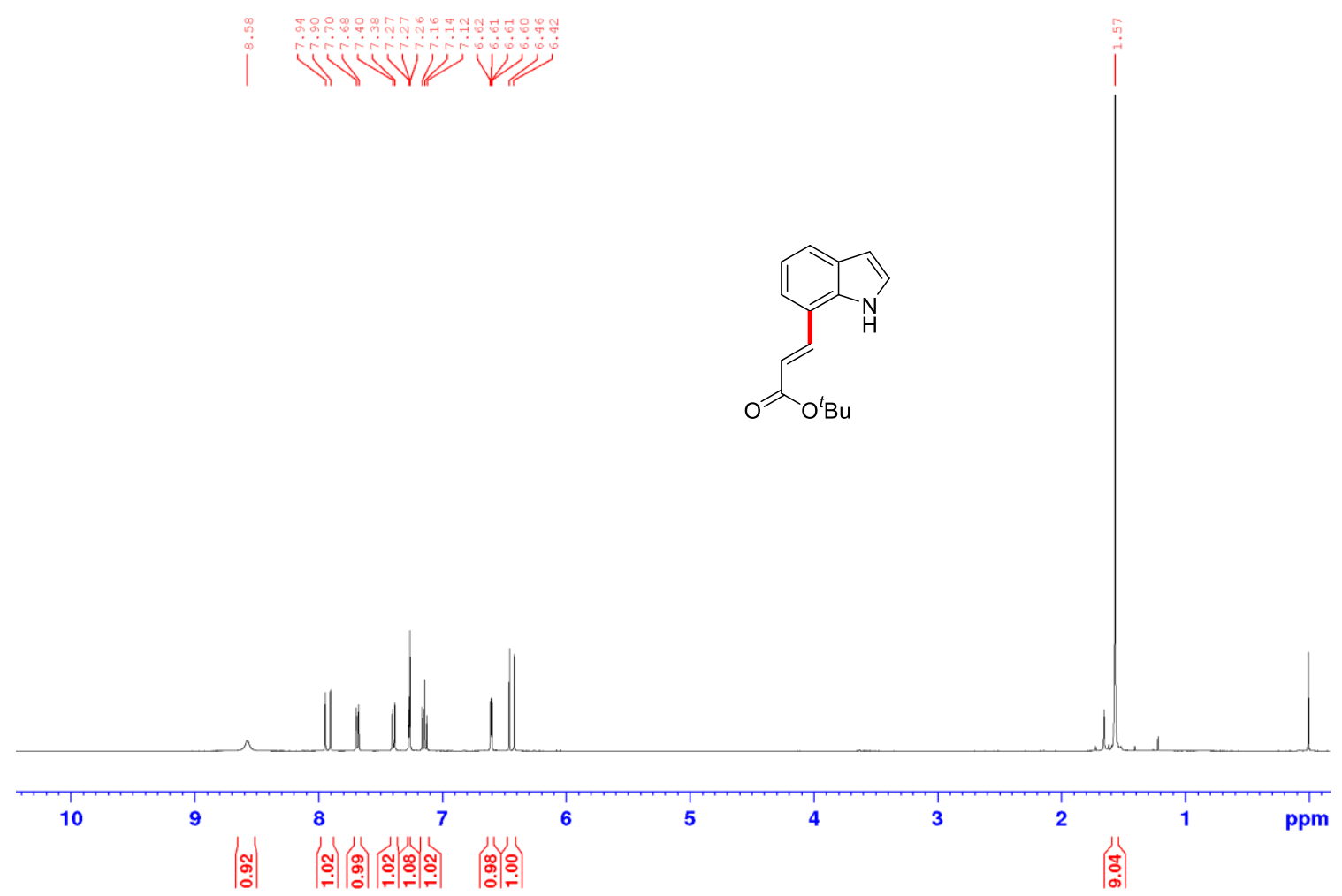

${ }^{13} \mathrm{C}$ NMR of 3be $\left(100 \mathrm{MHz}, \mathrm{CDCl}_{3}\right)$
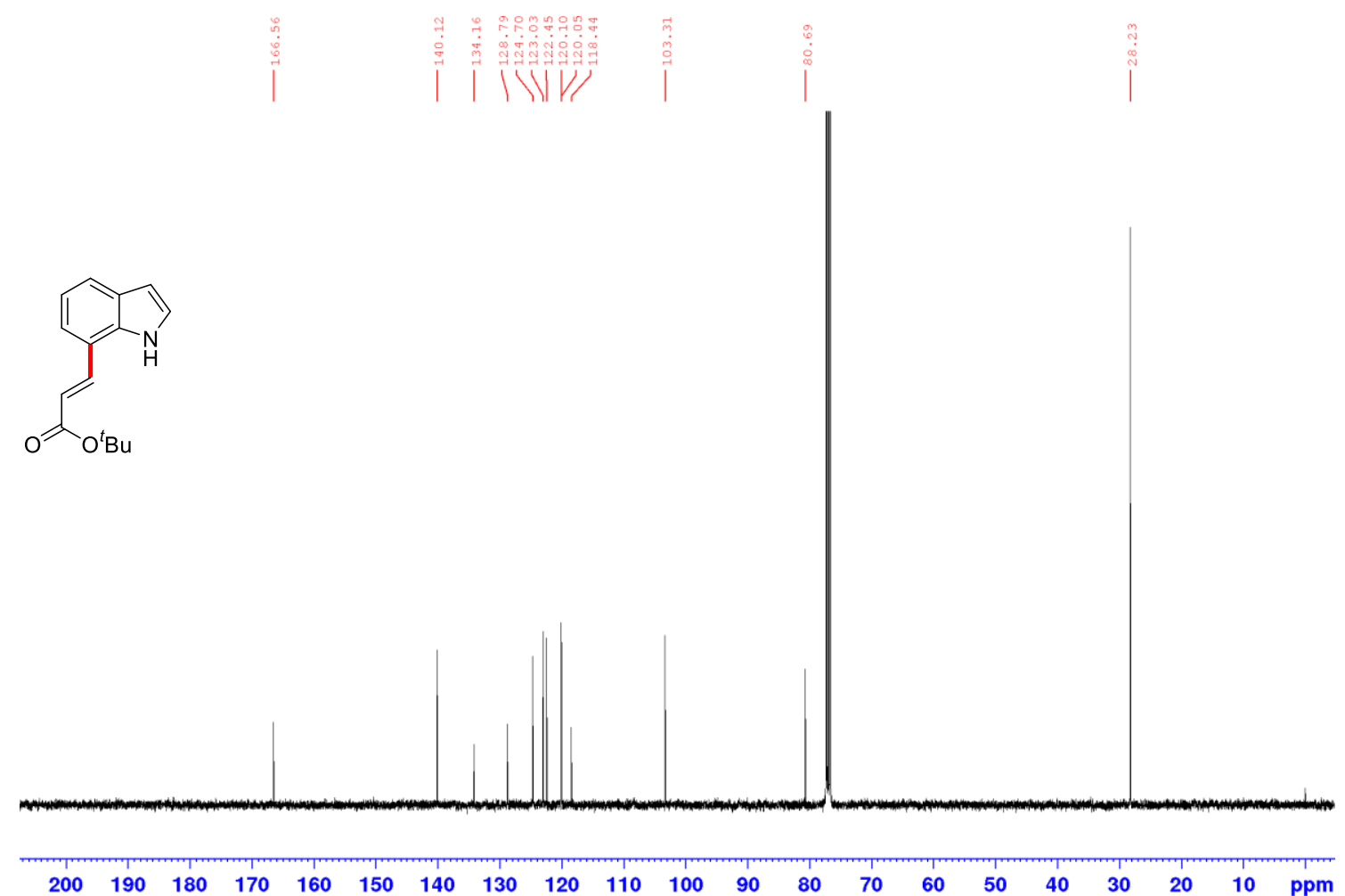
${ }^{1} \mathrm{H}$ NMR of $3 \mathbf{b f}\left(400 \mathrm{MHz}, \mathrm{CDCl}_{3}\right)$<smiles>O=C(/C=C/c1cccc2cc[nH]c12)OC1CCCCC1</smiles>
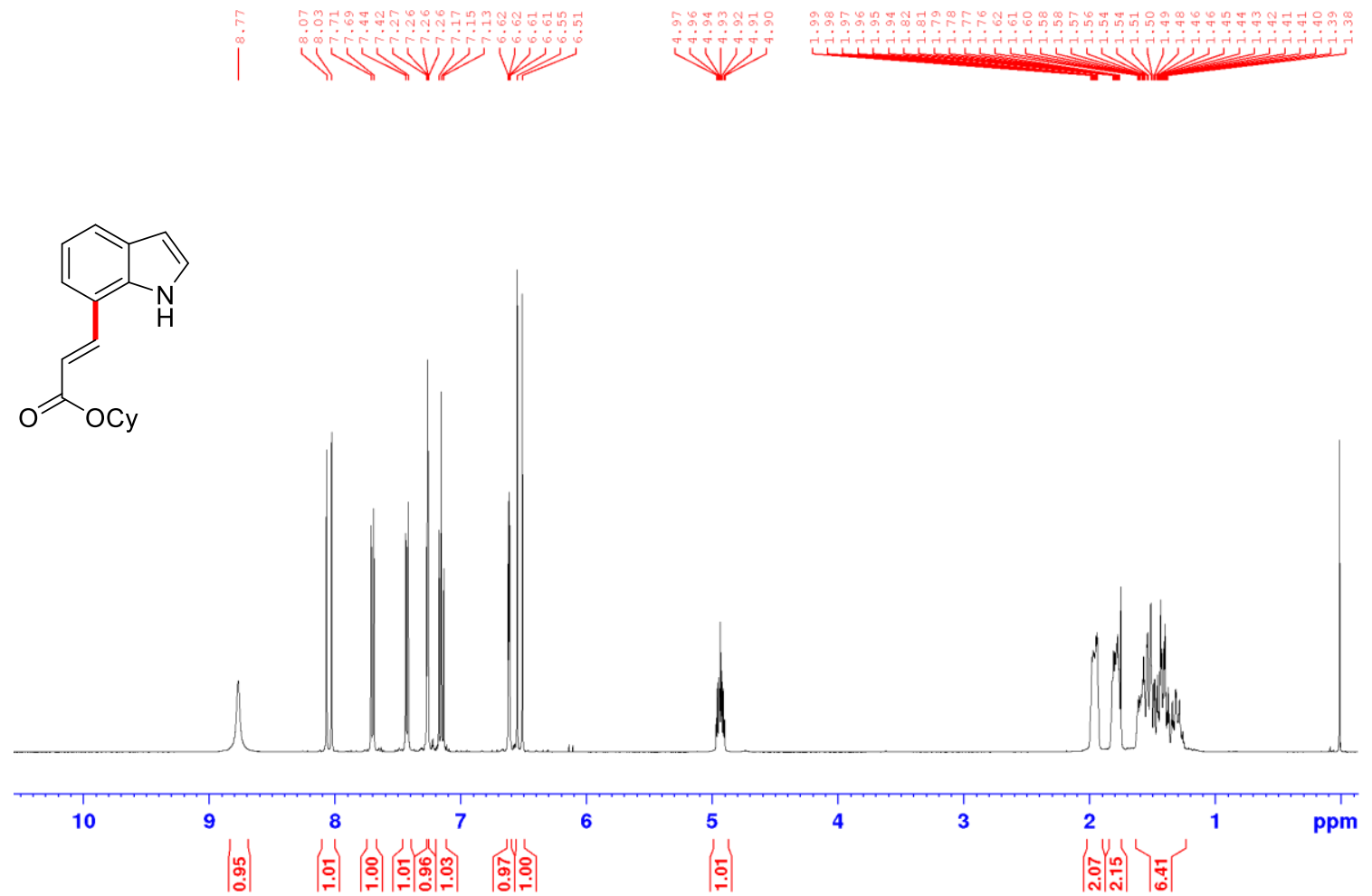

${ }^{13} \mathrm{C}$ NMR of $\mathbf{3 b f}\left(100 \mathrm{MHz}, \mathrm{CDCl}_{3}\right)$<smiles>O=C(/C=C/c1cccc2cc[nH]c12)OCCO</smiles>

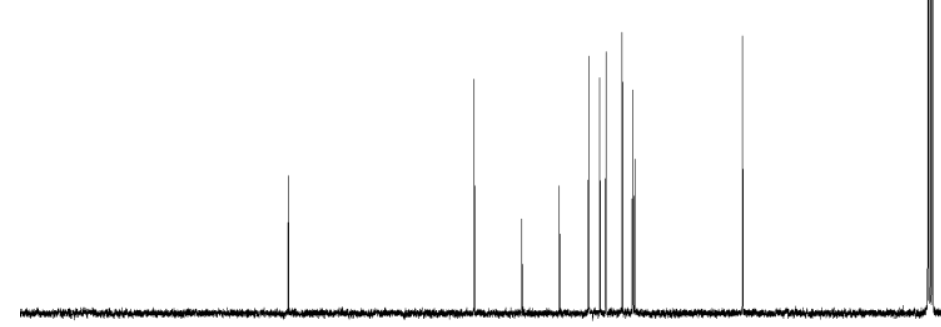


${ }^{1} \mathrm{H}$ NMR of $\mathbf{3 b g}\left(400 \mathrm{MHz}, \mathrm{CDCl}_{3}\right)$

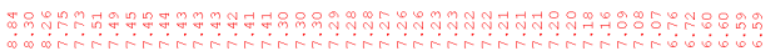

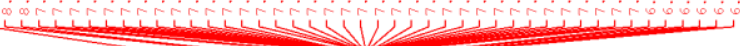

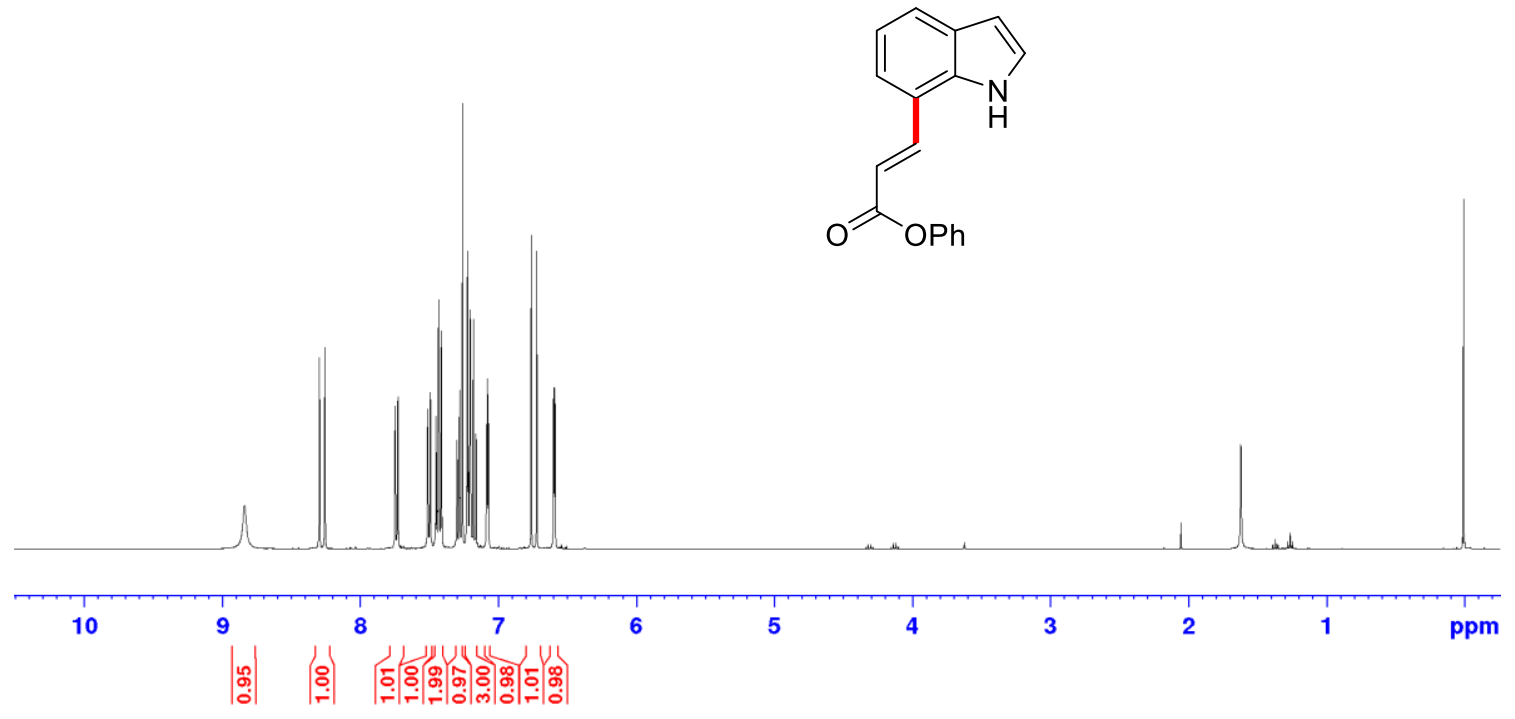

${ }^{13} \mathrm{C} \mathrm{NMR}$ of $\mathbf{3 b g}\left(100 \mathrm{MHz}, \mathrm{CDCl}_{3}\right)$

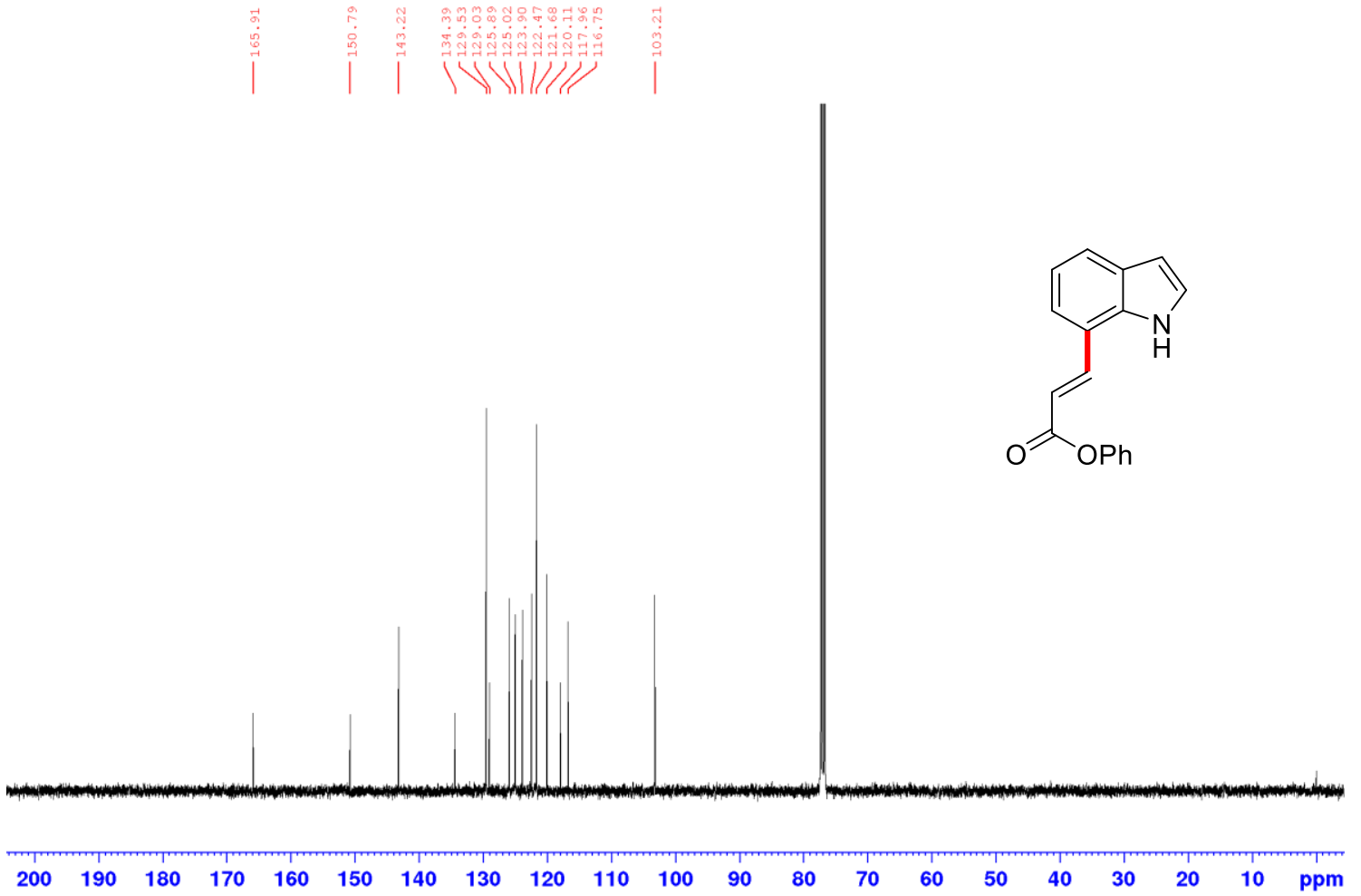


${ }^{1} \mathrm{H}$ NMR of $\mathbf{3 b h}\left(400 \mathrm{MHz}, \mathrm{CDCl}_{3}\right)$

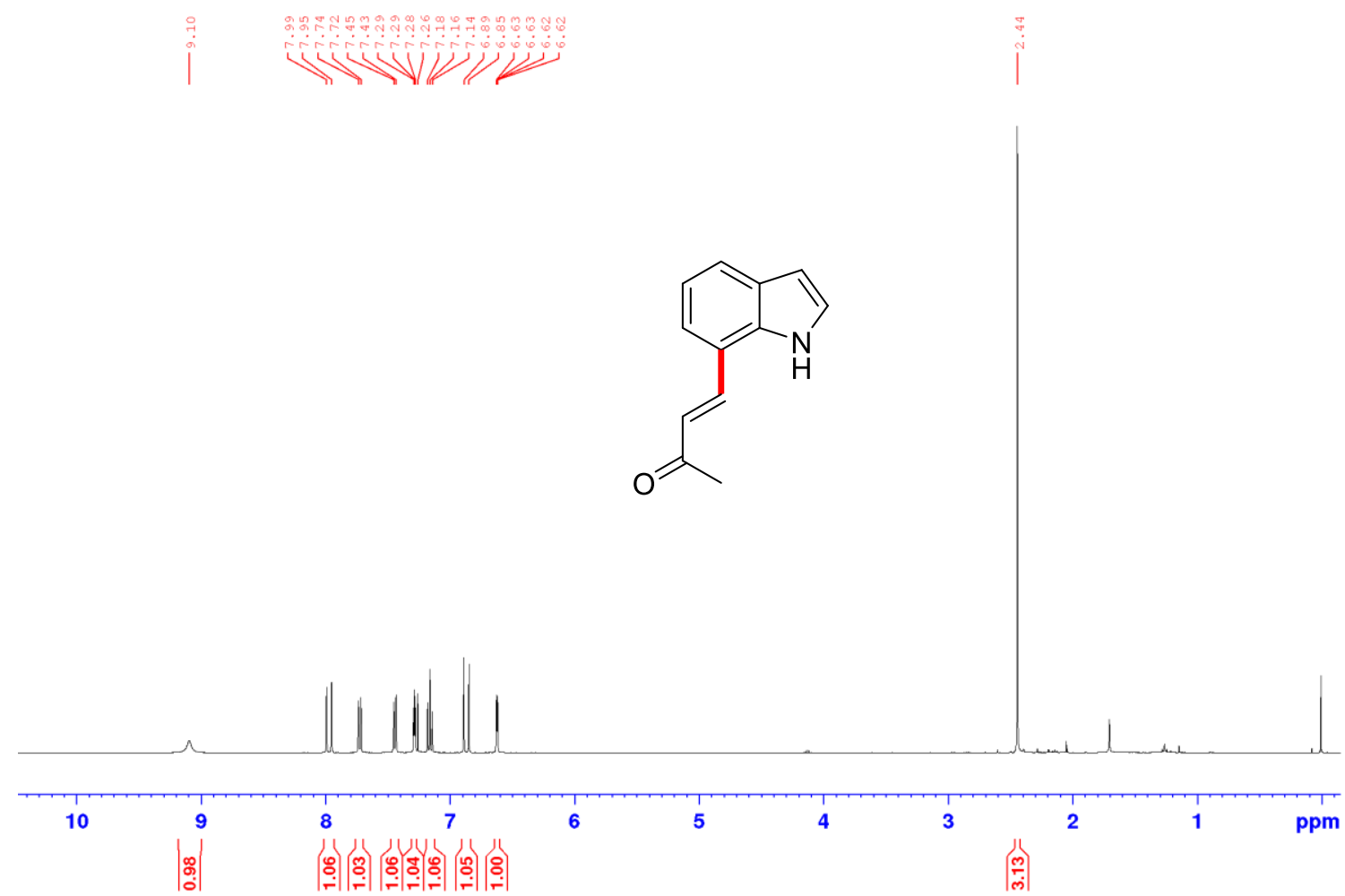

${ }^{13} \mathrm{C}$ NMR of $\mathbf{3 b h}\left(100 \mathrm{MHz}, \mathrm{CDCl}_{3}\right)$

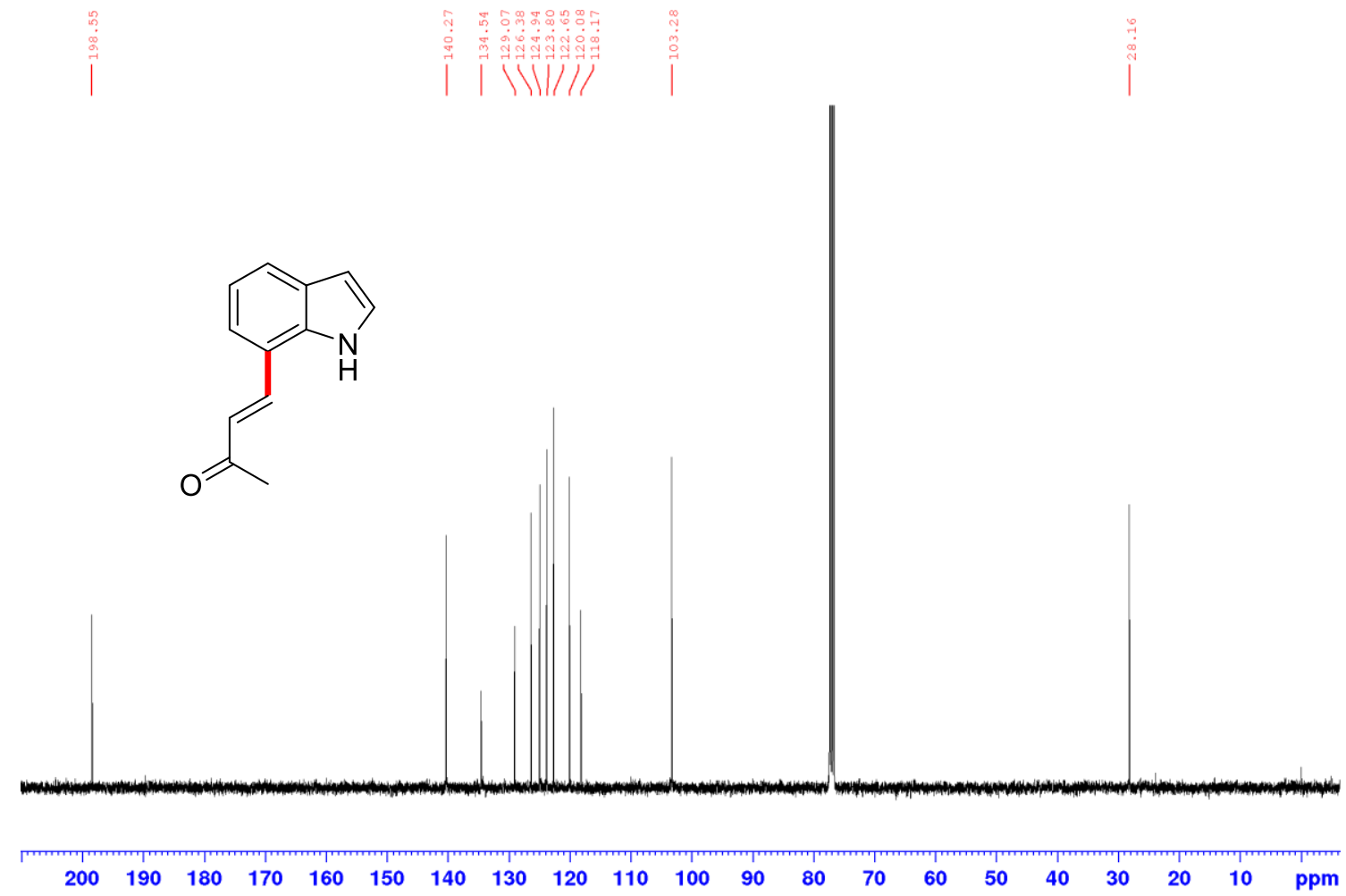


${ }^{1} \mathrm{H}$ NMR of 3bi $\left(400 \mathrm{MHz}, \mathrm{CDCl}_{3}\right)$
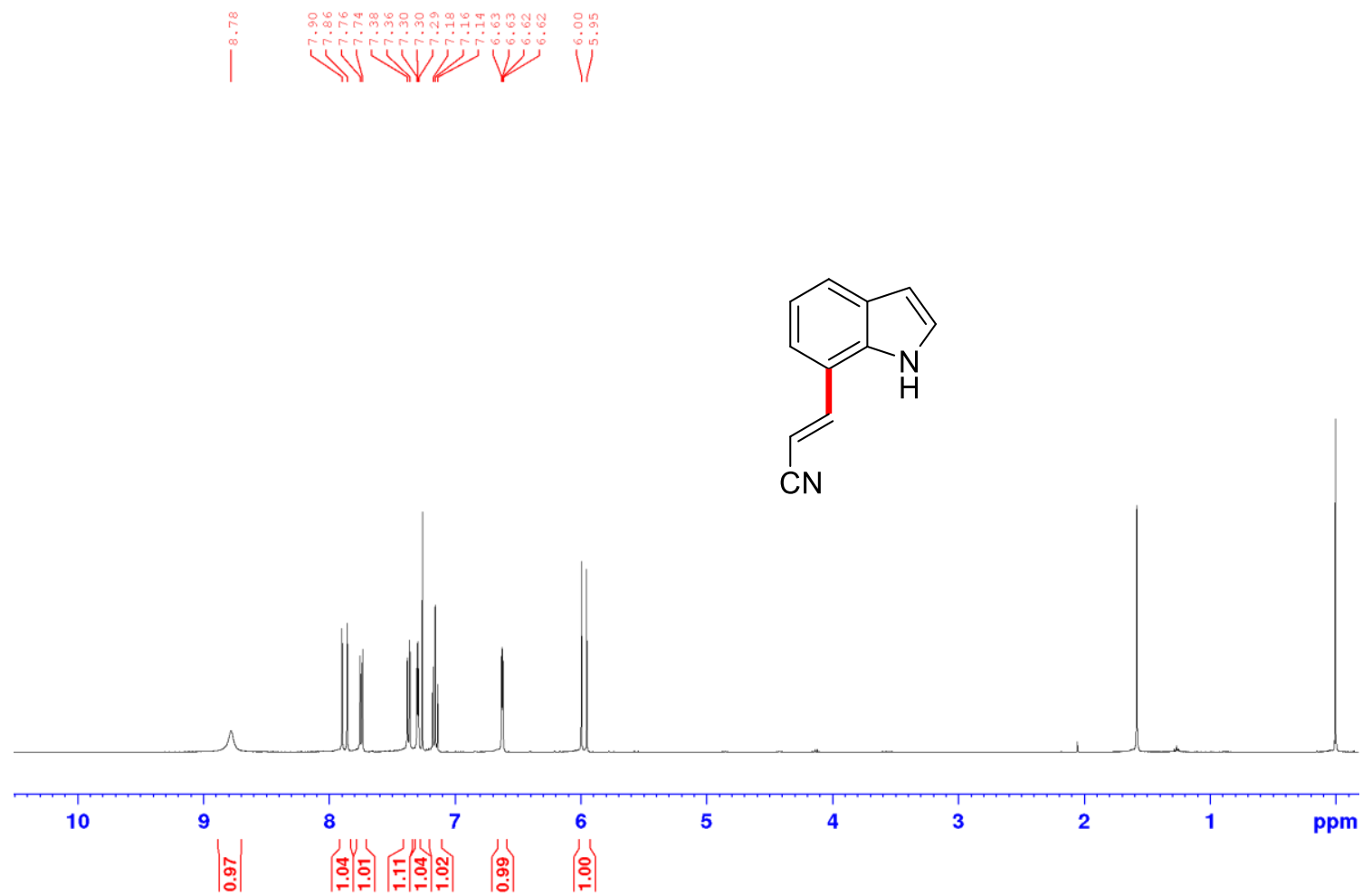

${ }^{13} \mathrm{C}$ NMR of 3 bi $\left(100 \mathrm{MHz}, \mathrm{CDCl}_{3}\right)$

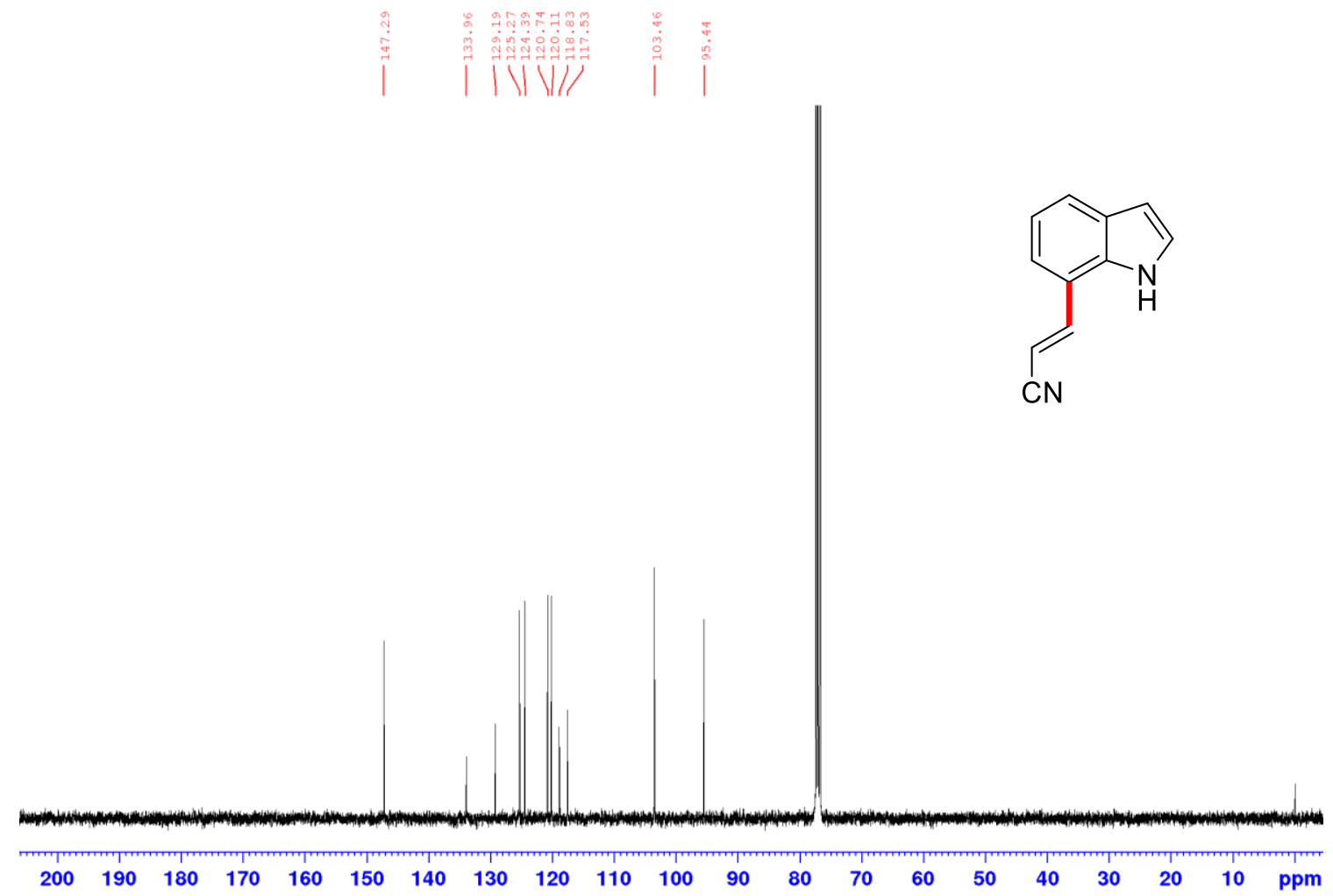


${ }^{1} \mathrm{H}$ NMR of $3 \mathbf{b j}\left(400 \mathrm{MHz}, \mathrm{CDCl}_{3}\right)$
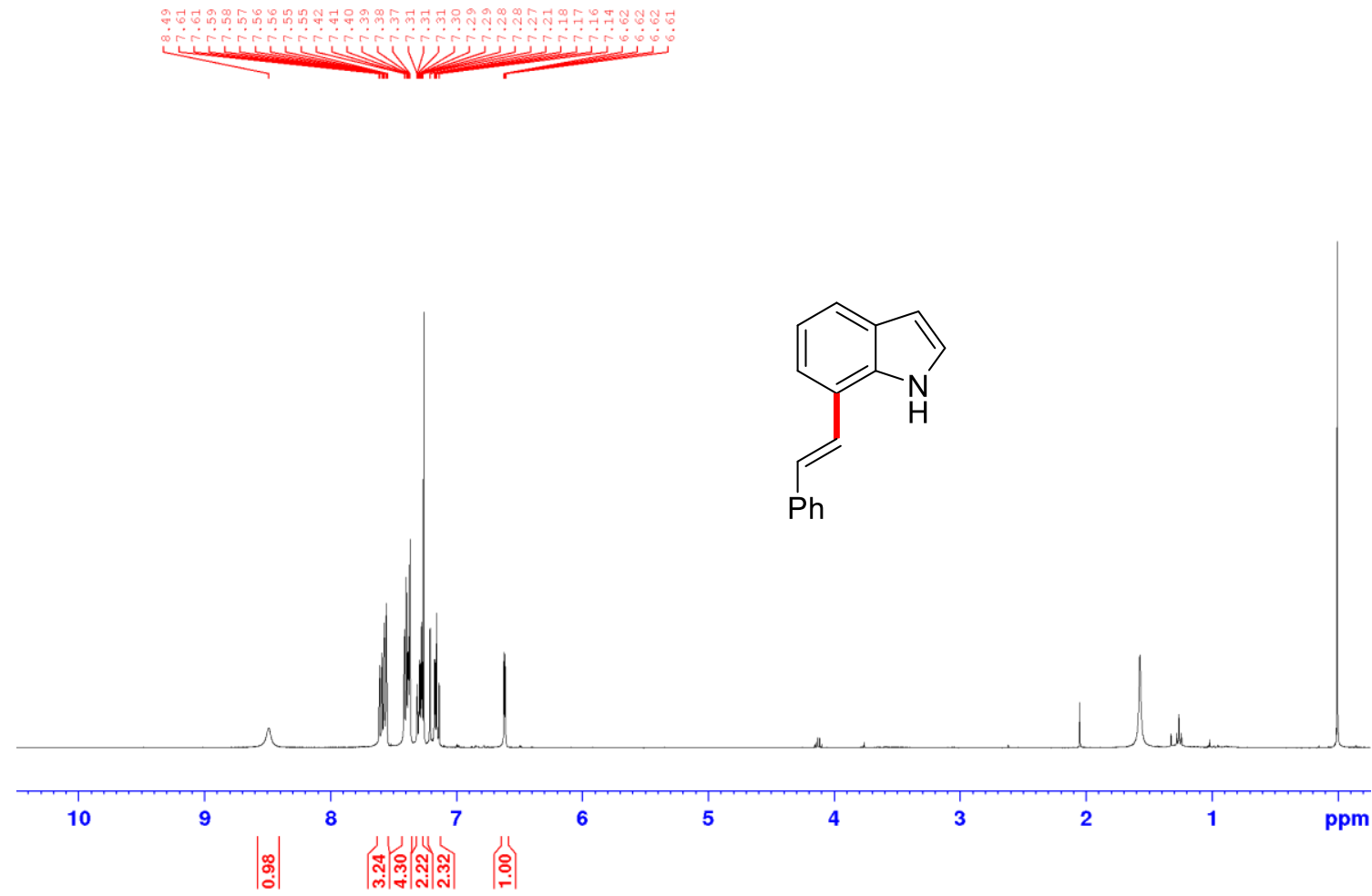

${ }^{13} \mathrm{C}$ NMR of $\mathbf{3 b j}\left(100 \mathrm{MHz}, \mathrm{CDCl}_{3}\right)$

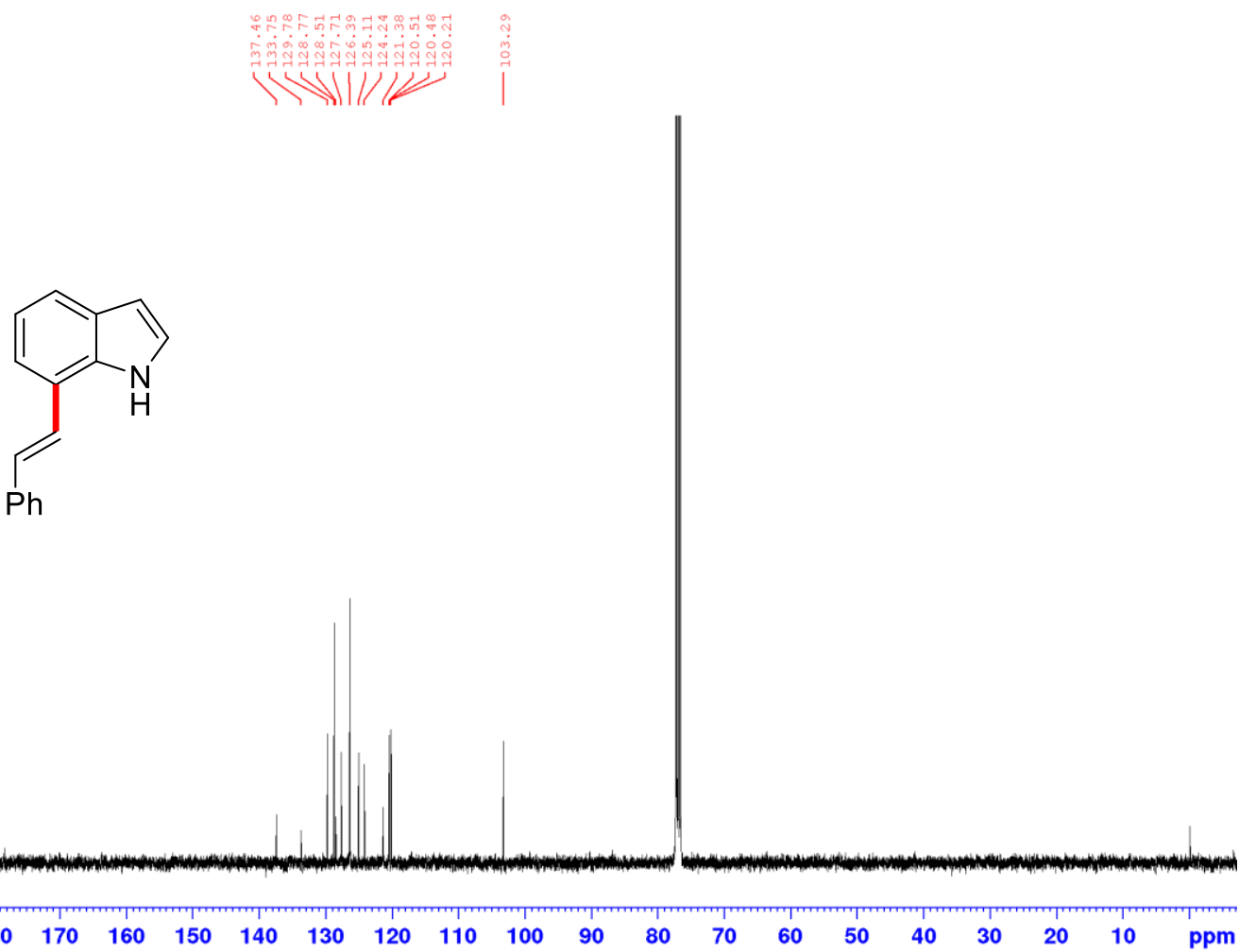


${ }^{1} \mathrm{H}$ NMR of 3da $\left(400 \mathrm{MHz}, \mathrm{CDCl}_{3}\right)$

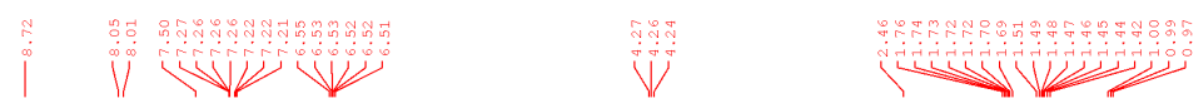<smiles>CCCCC(=O)/C=C/c1cc(C)cc2cc[nH]c12</smiles>

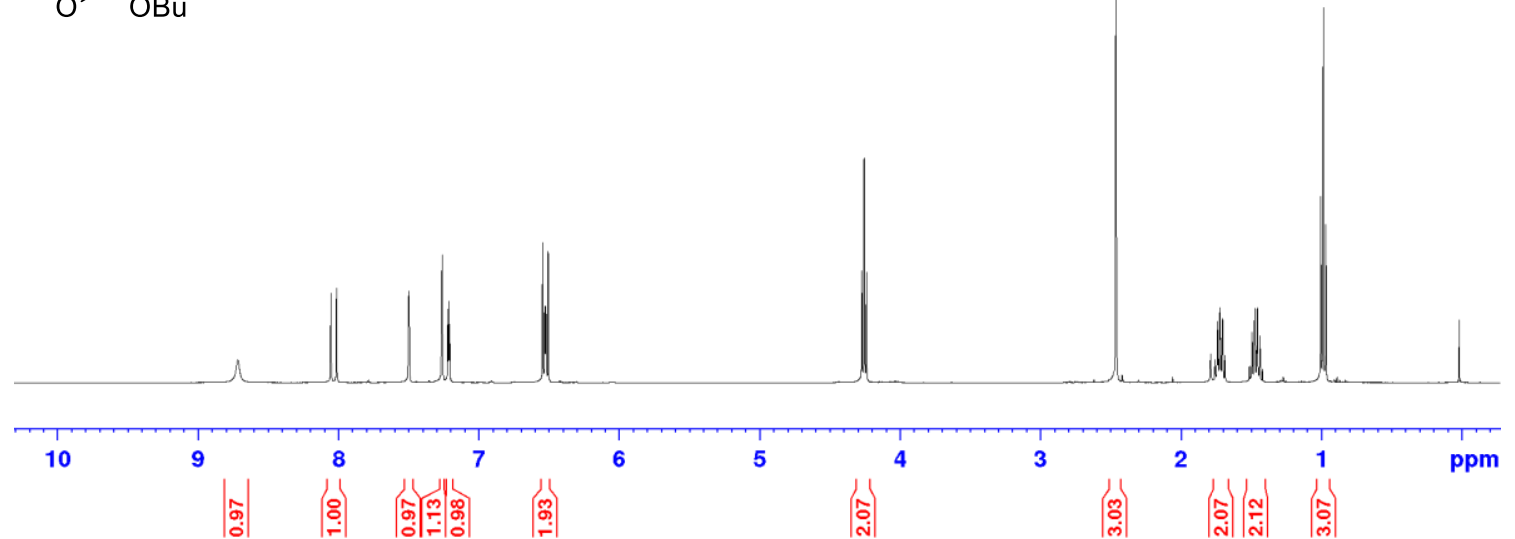

${ }^{13} \mathrm{C} \mathrm{NMR}$ of 3da $\left(100 \mathrm{MHz}, \mathrm{CDCl}_{3}\right)$

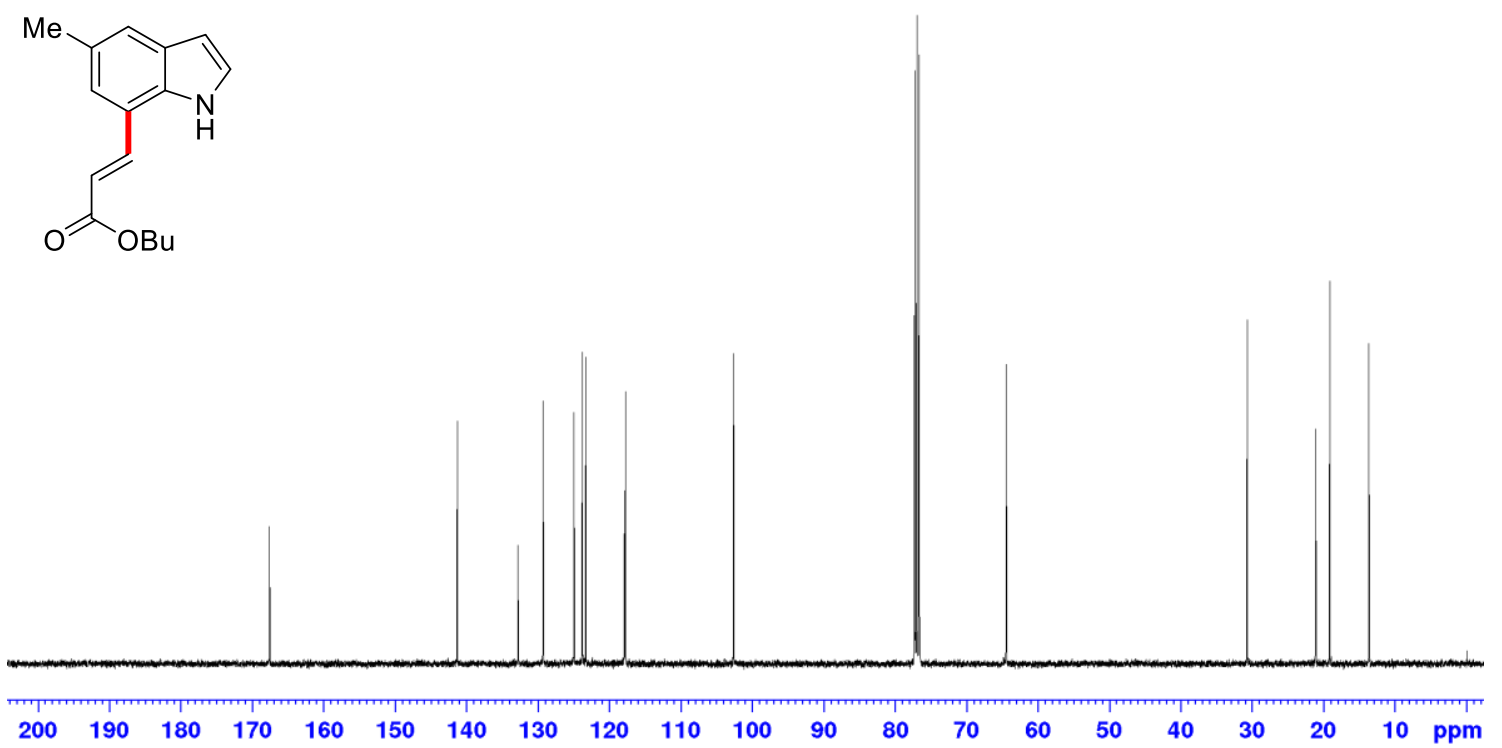


${ }^{1} \mathrm{H}$ NMR of $3 \mathbf{e a}\left(400 \mathrm{MHz}, \mathrm{CDCl}_{3}\right)$

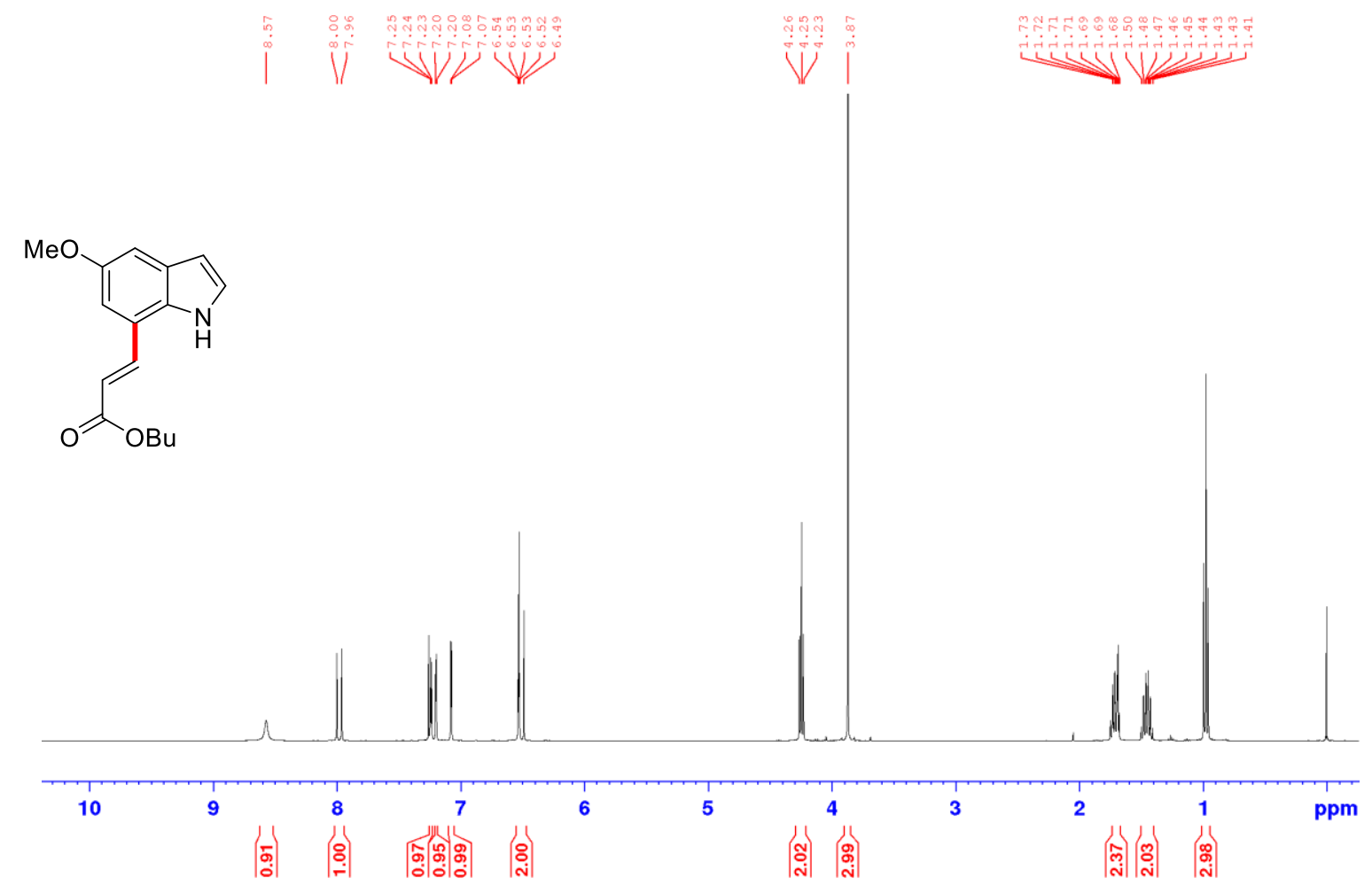

${ }^{13} \mathrm{C}$ NMR of 3ea $\left(100 \mathrm{MHz}, \mathrm{CDCl}_{3}\right)$

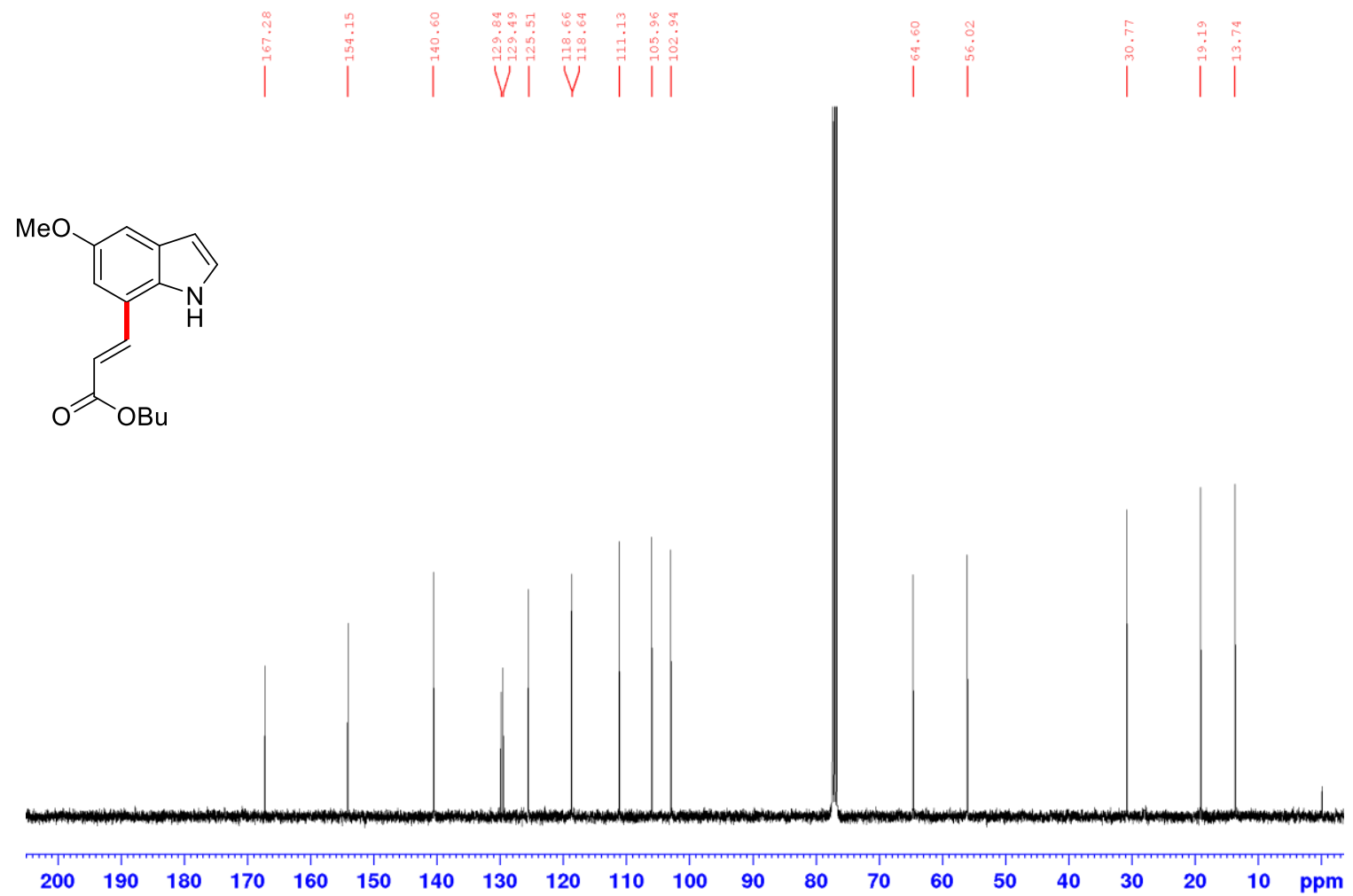


${ }^{1} \mathrm{H}$ NMR of $3 f a\left(400 \mathrm{MHz}, \mathrm{CDCl}_{3}\right)$

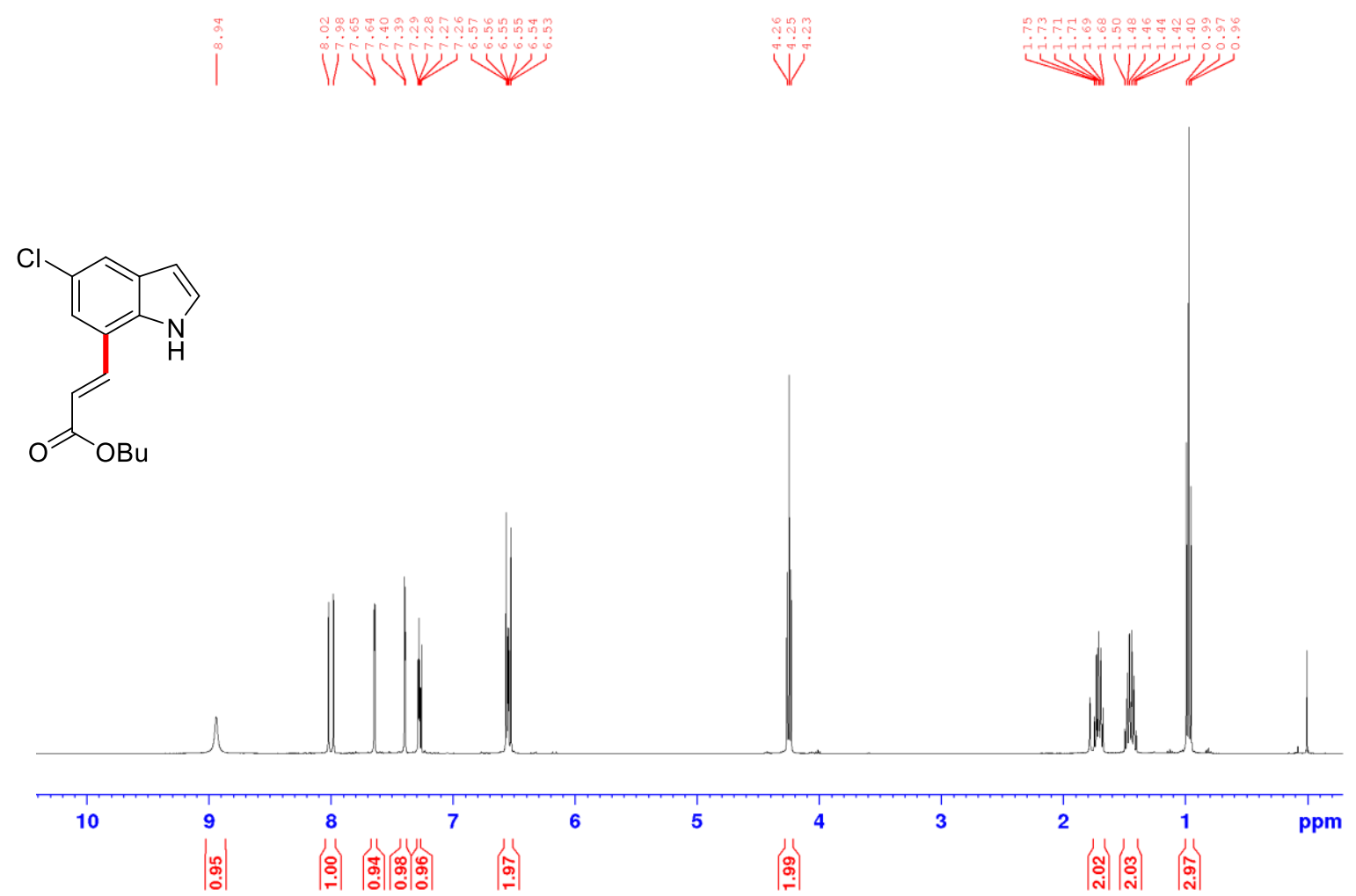

${ }^{13} \mathrm{C}$ NMR of $\mathbf{3 f a}\left(100 \mathrm{MHz}, \mathrm{CDCl}_{3}\right)$

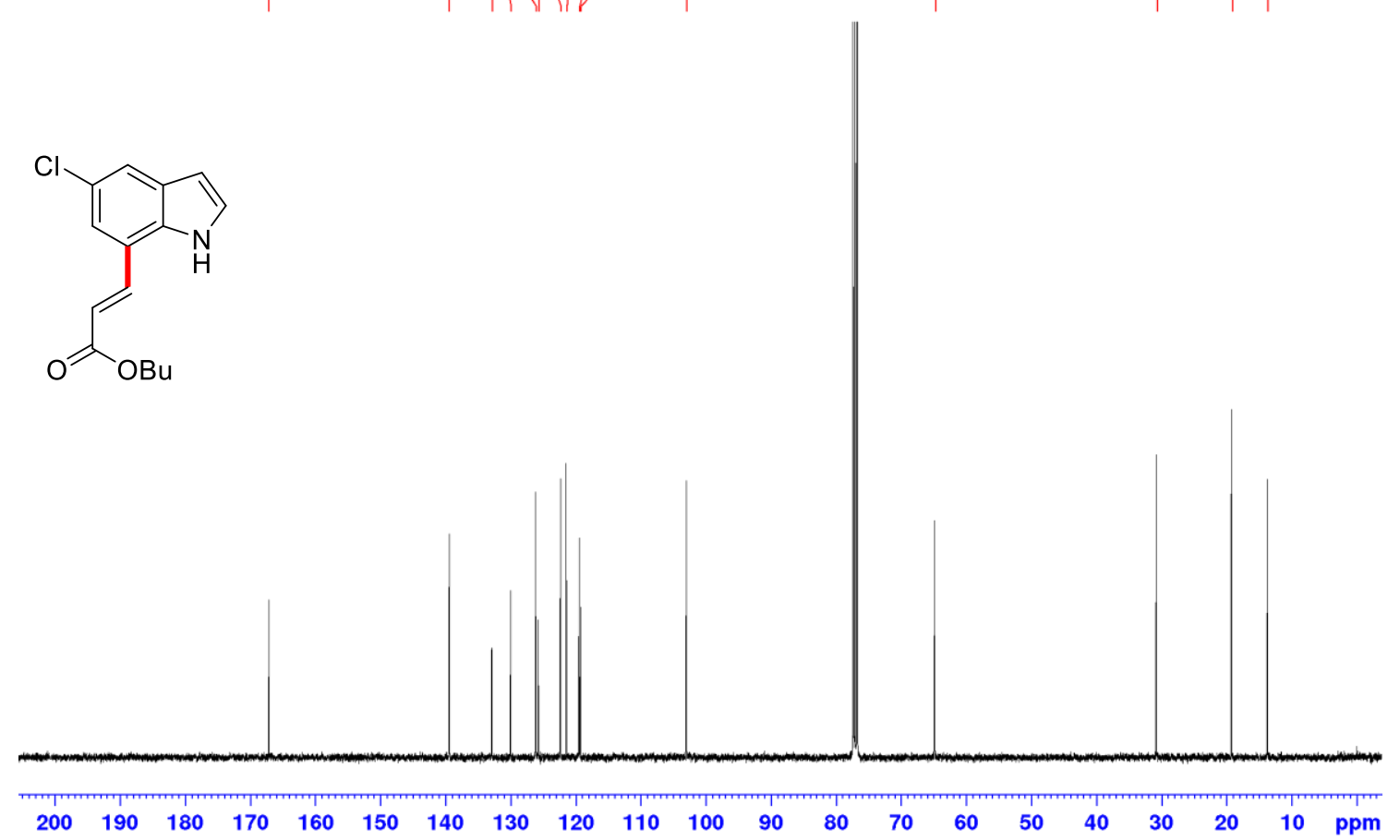


${ }^{1} \mathrm{H}$ NMR of $3 g a\left(400 \mathrm{MHz}, \mathrm{CDCl}_{3}\right)$

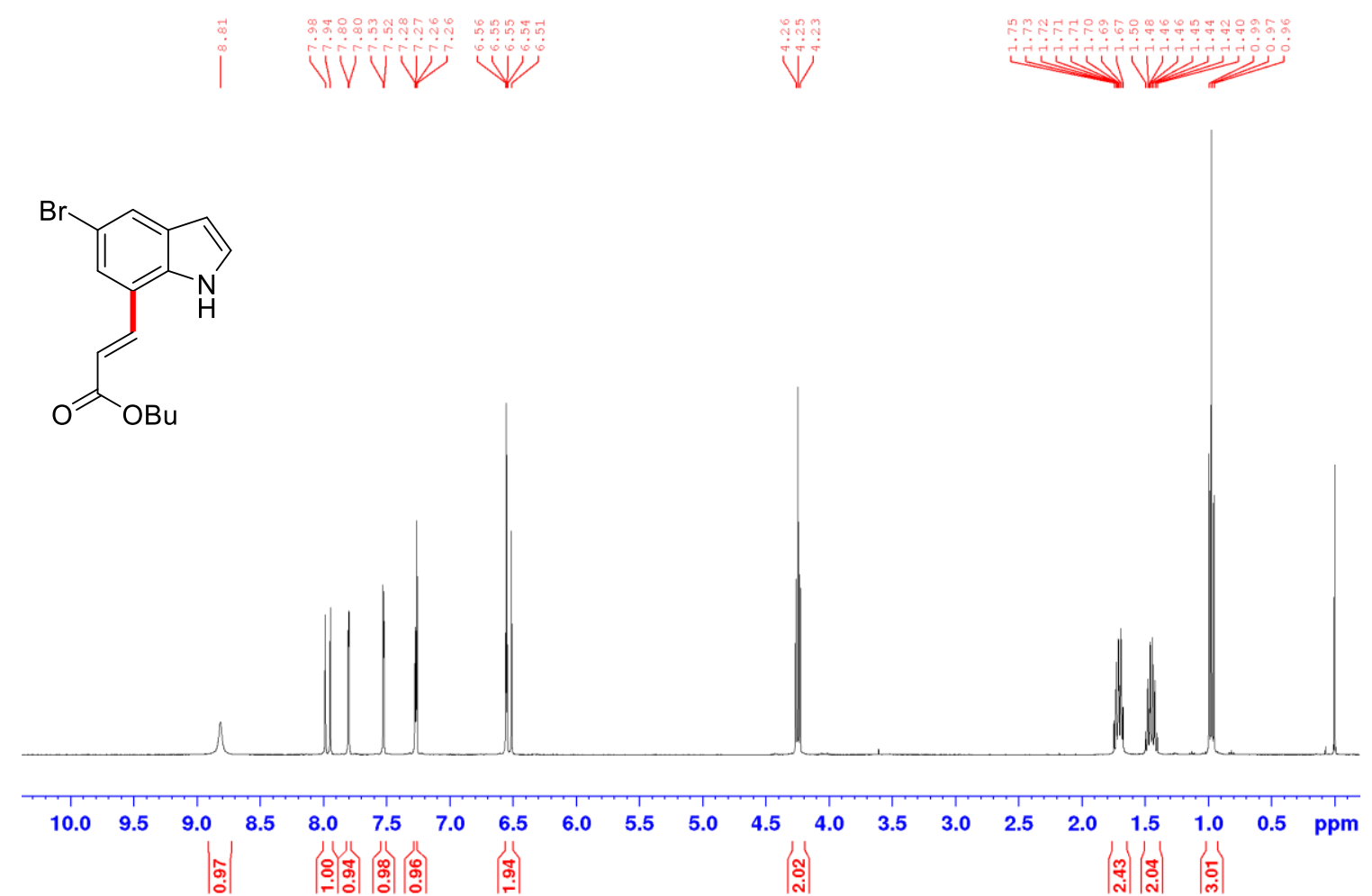

${ }^{13} \mathrm{C} \mathrm{NMR}$ of 3 ga $\left(100 \mathrm{MHz}, \mathrm{CDCl}_{3}\right)$

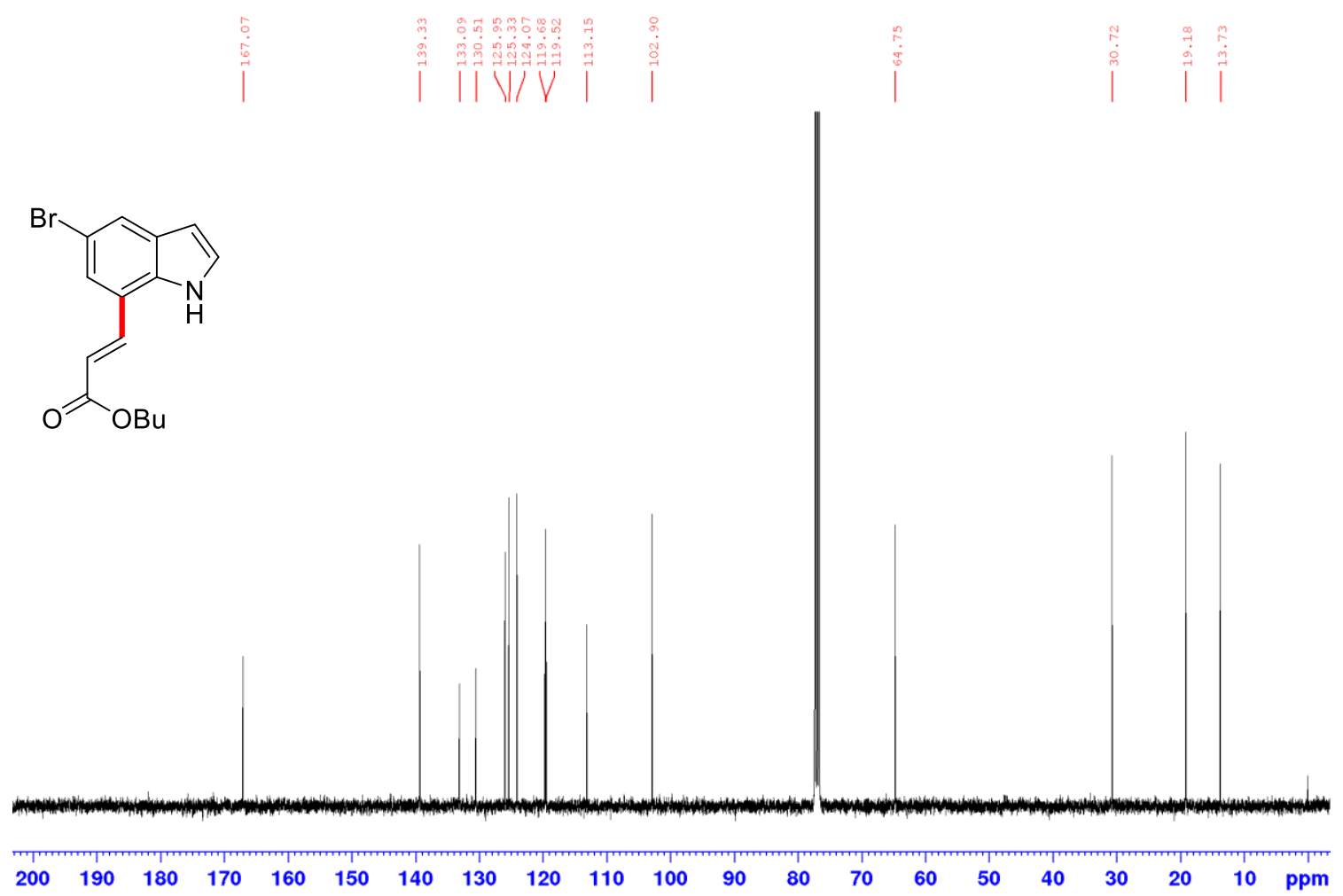


${ }^{1} \mathrm{H}$ NMR of 3 ha $\left(400 \mathrm{MHz}, \mathrm{CDCl}_{3}\right)$

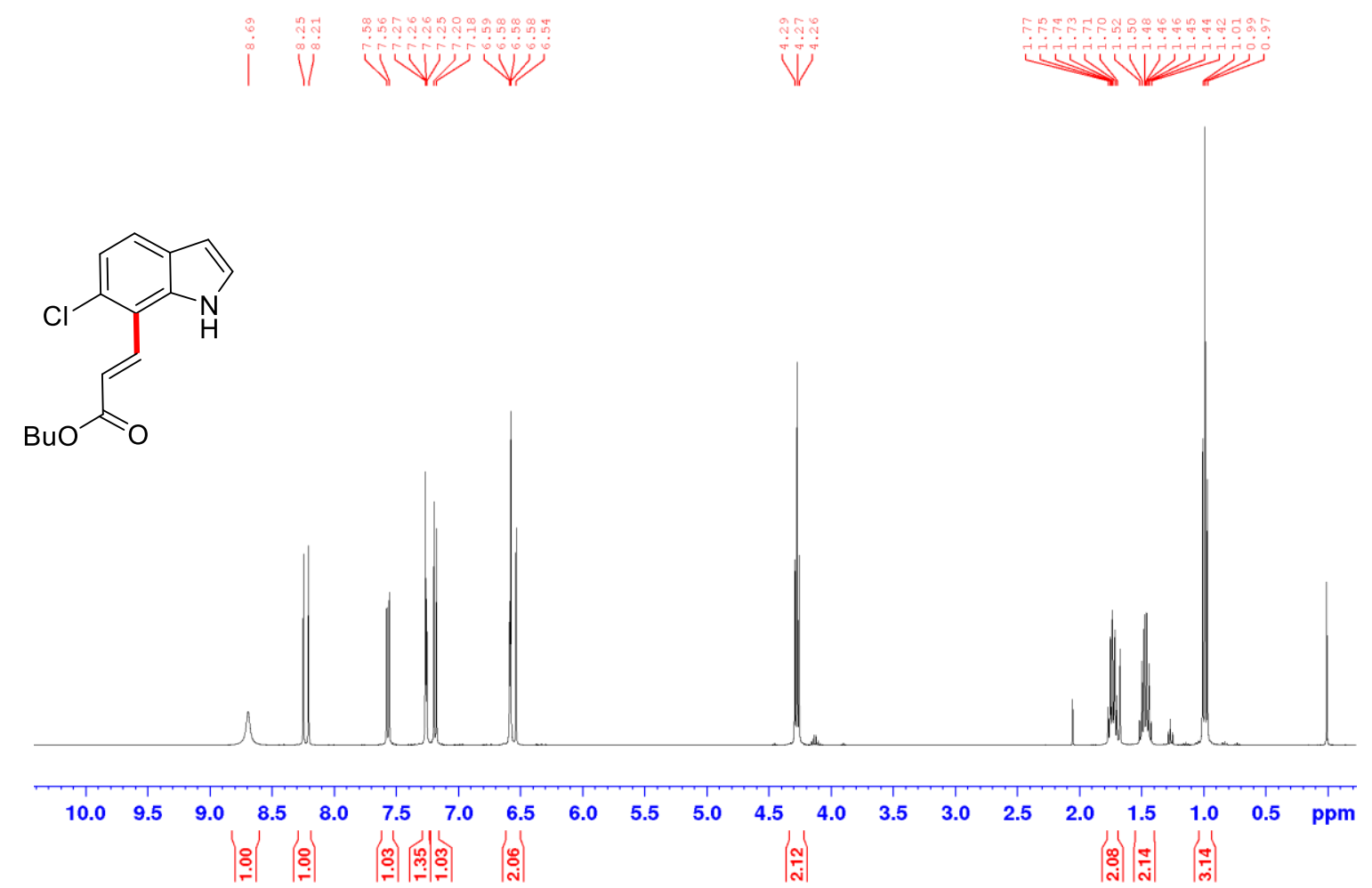

${ }^{13} \mathrm{C} \mathrm{NMR}$ of $3 \mathbf{h a}\left(100 \mathrm{MHz}, \mathrm{CDCl}_{3}\right)$

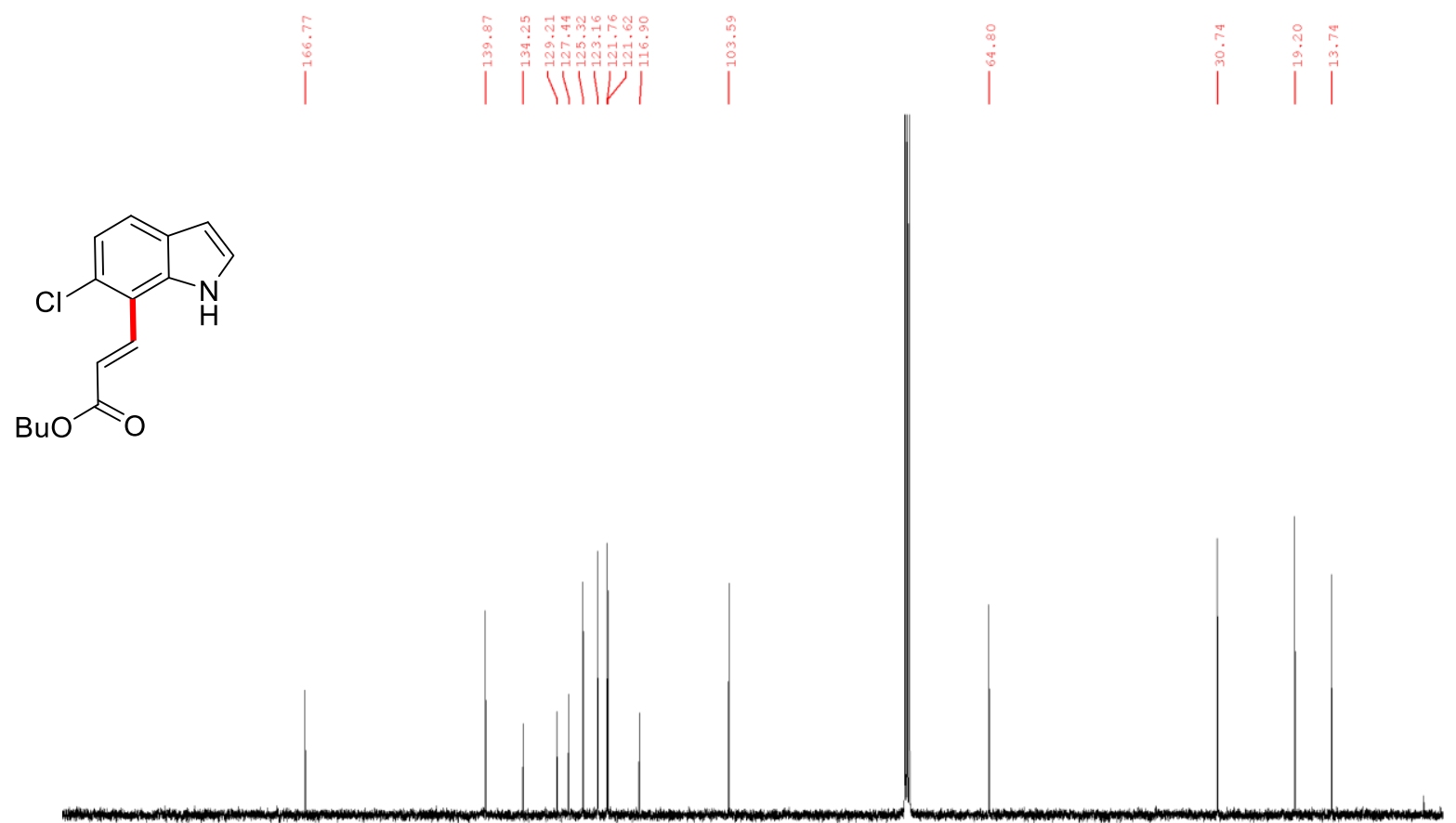

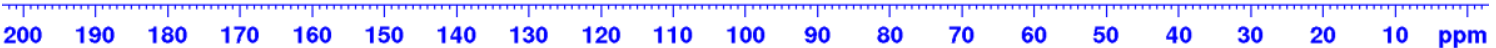


${ }^{1} \mathrm{H}$ NMR of $3 i a\left(400 \mathrm{MHz}, \mathrm{CDCl}_{3}\right)$

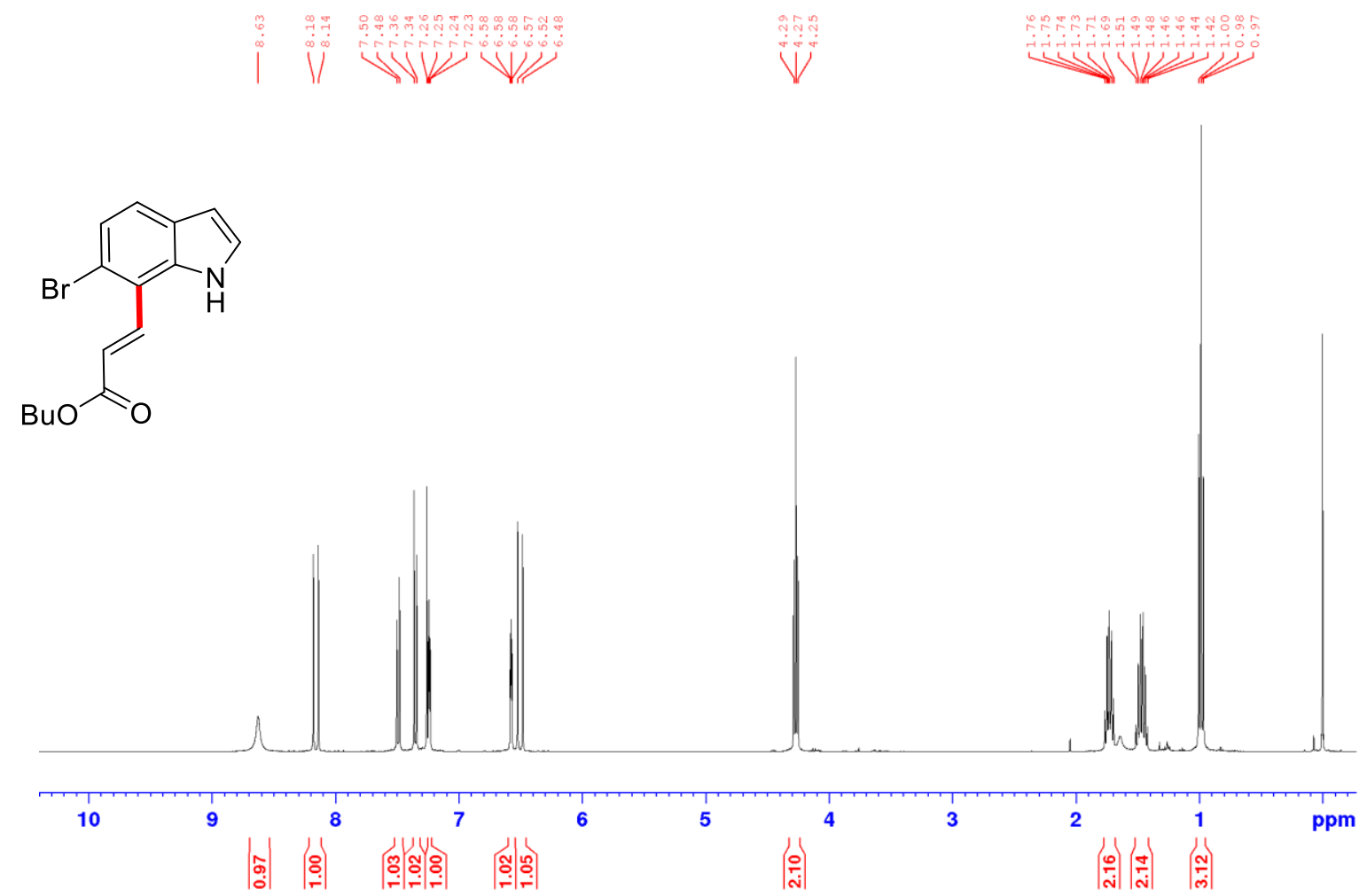

${ }^{13} \mathrm{C}$ NMR of 3ia $\left(100 \mathrm{MHz}, \mathrm{CDCl}_{3}\right)$

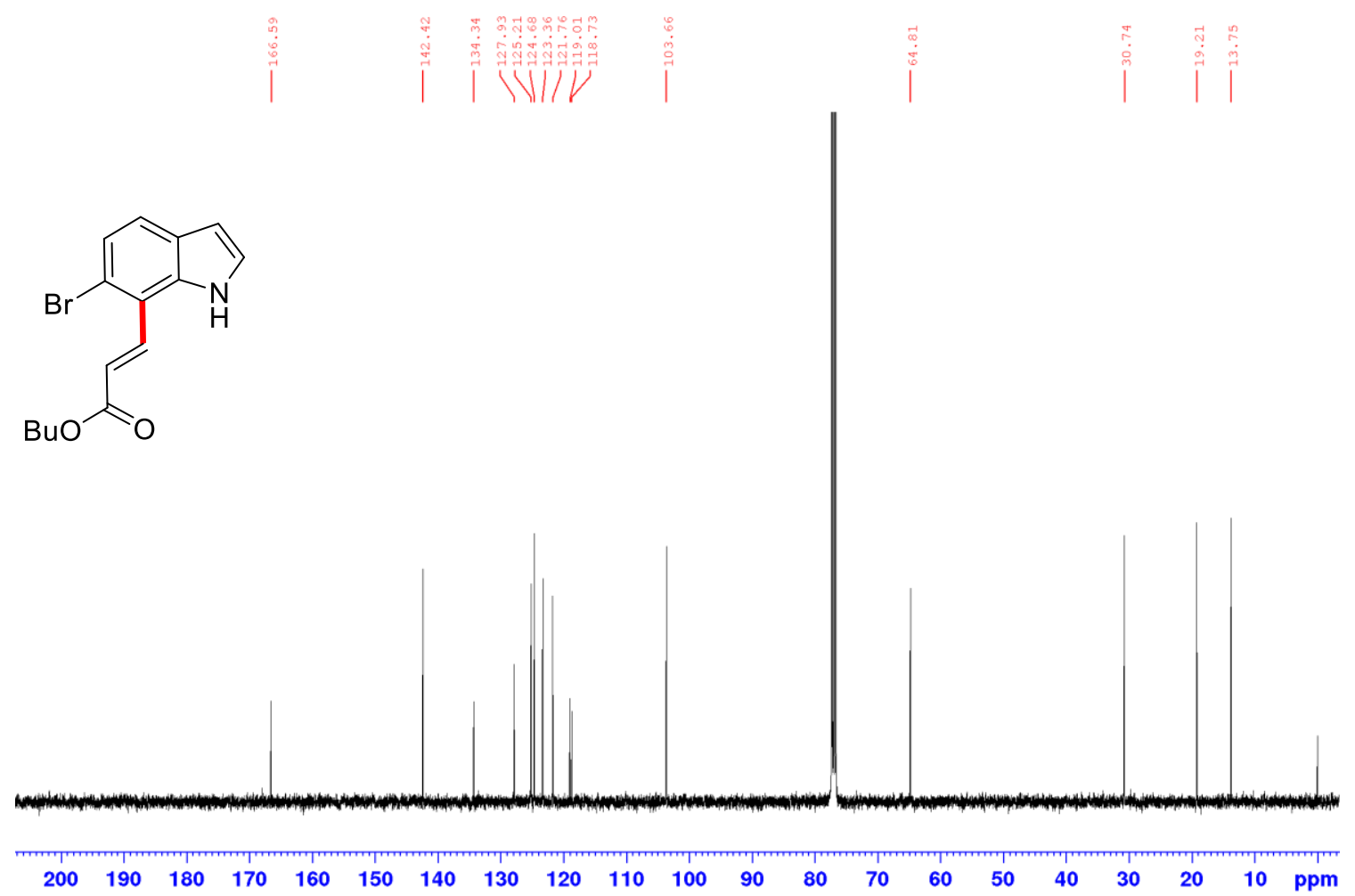


${ }^{1} \mathrm{H}$ NMR of 3ka (400 MHz, $\left.\mathrm{CDCl}_{3}\right)$

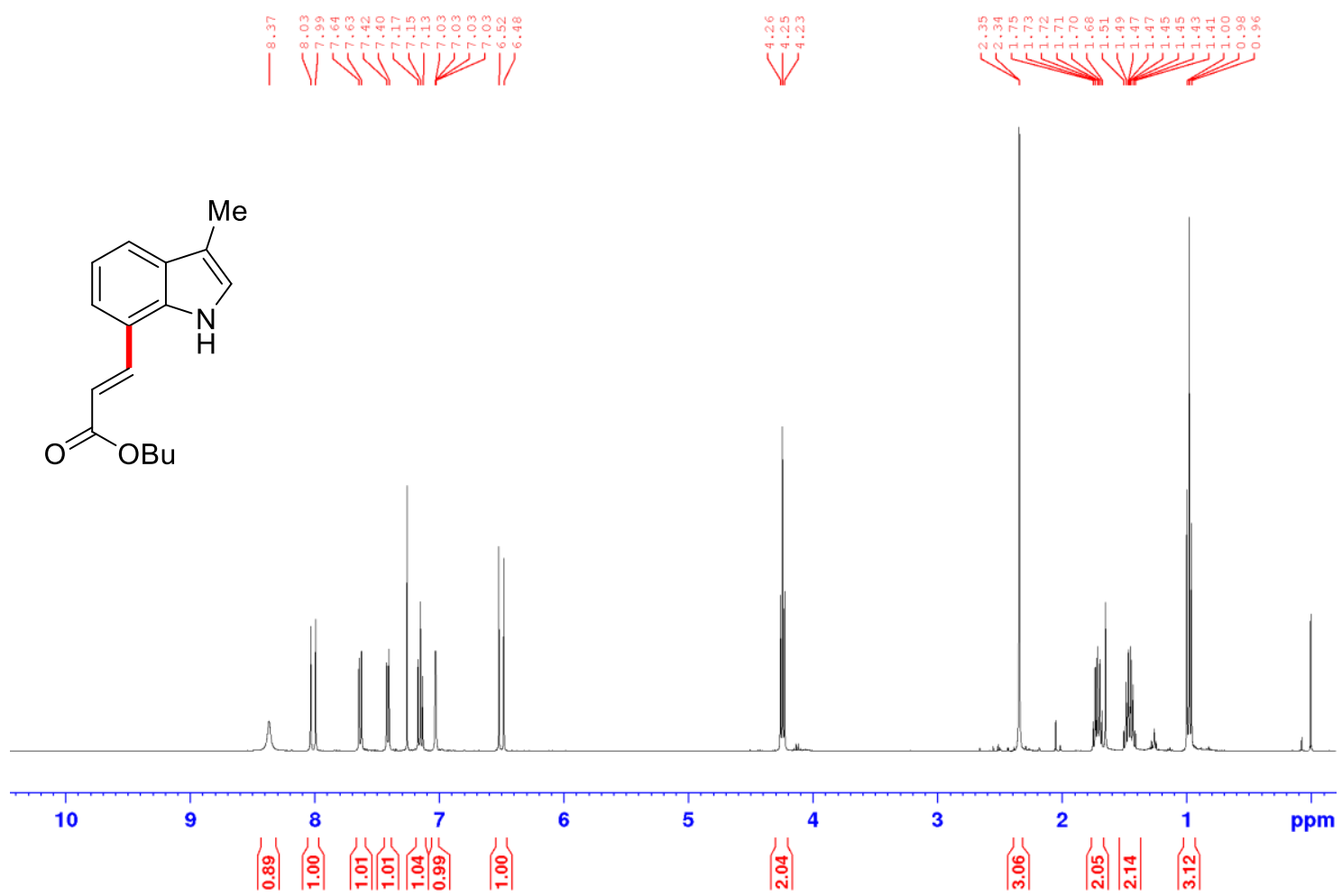

${ }^{13} \mathrm{C}$ NMR of 3ka $\left(100 \mathrm{MHz}, \mathrm{CDCl}_{3}\right)$

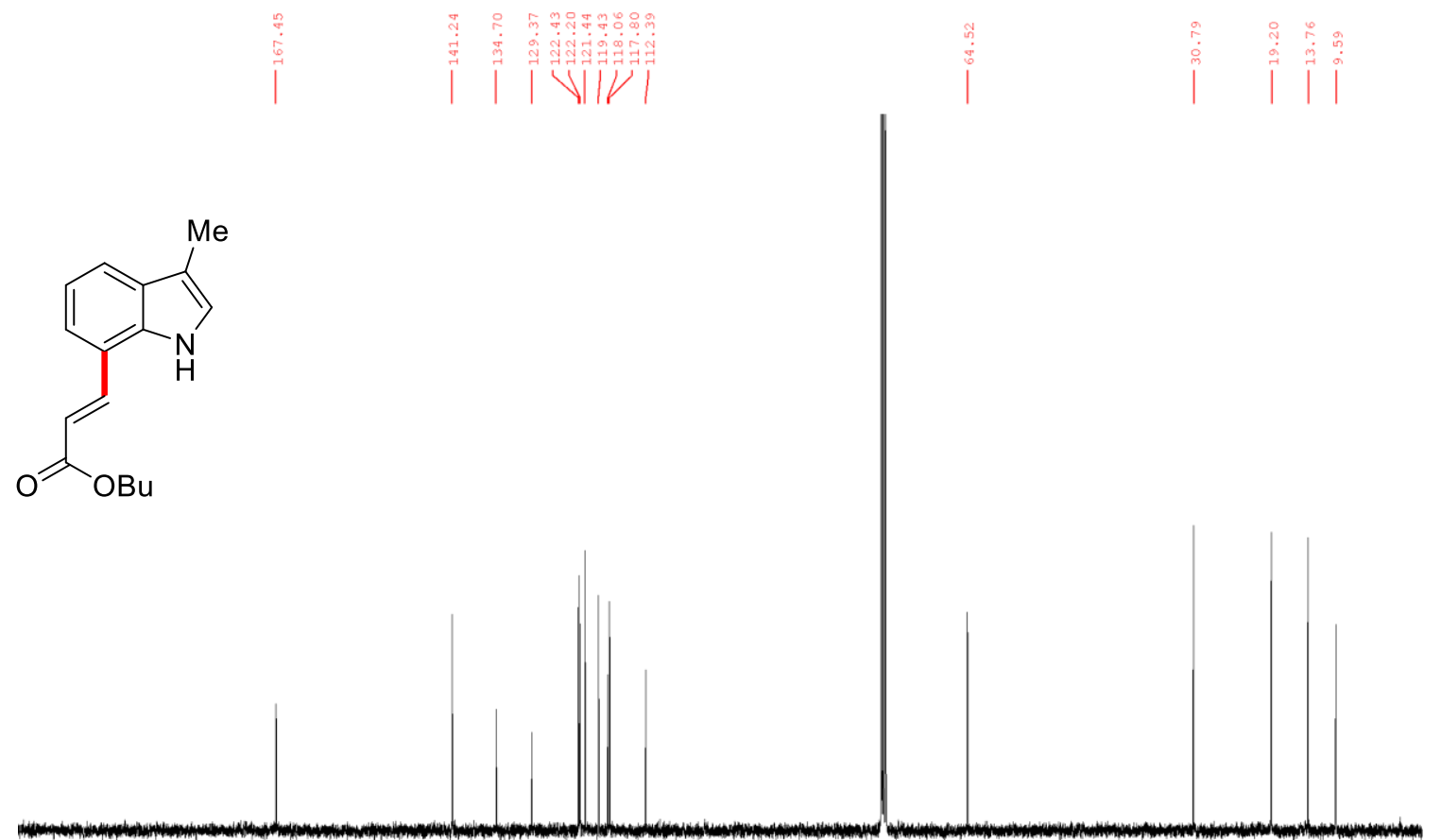


${ }^{1} \mathrm{H}$ NMR of $3 \mathbf{a}\left(400 \mathrm{MHz}, \mathrm{CDCl}_{3}\right)$
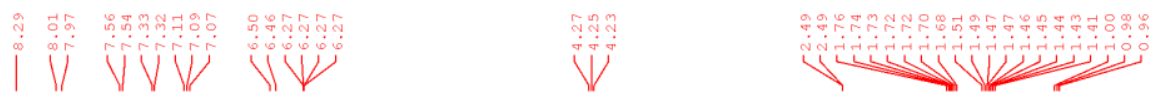<smiles>CCC(C)OC(=O)/C=C/c1cccc2cc(C)[nH]c12</smiles>

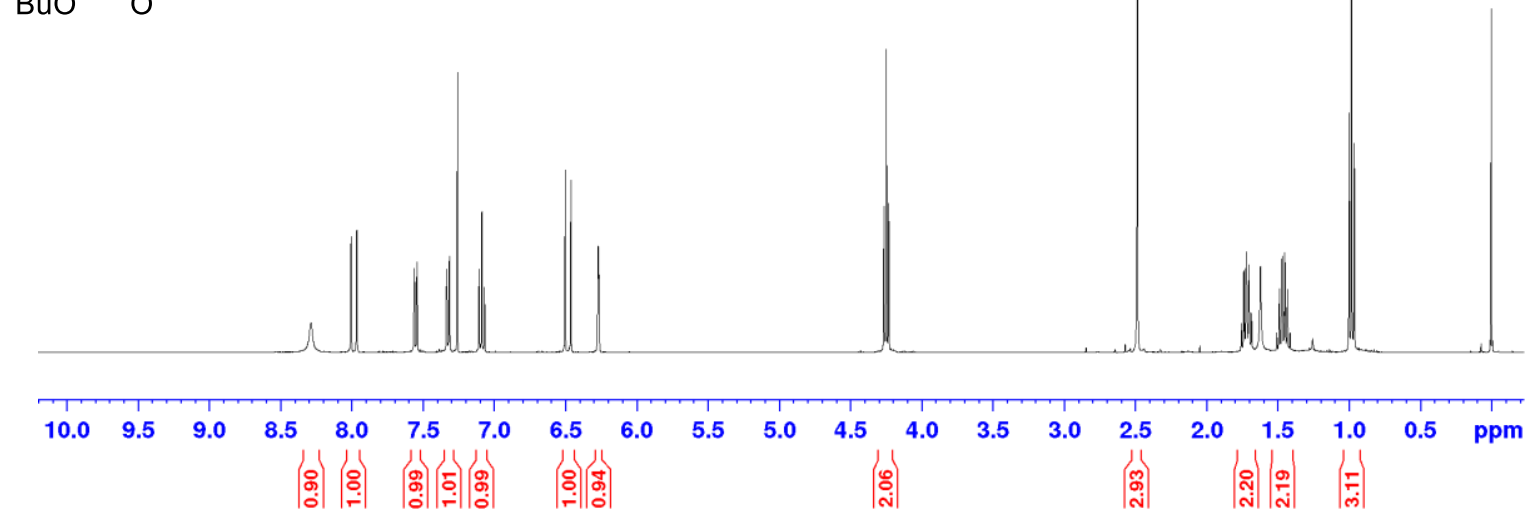

${ }^{13} \mathrm{C}$ NMR of 3la $\left(100 \mathrm{MHz}, \mathrm{CDCl}_{3}\right)$<smiles>Cc1cc2cccc(/C=C/C(=O)OC(C)(C)C)c2[nH]1</smiles> 
${ }^{1} \mathrm{H}$ NMR of $\mathbf{3 m a}\left(400 \mathrm{MHz}, \mathrm{CDCl}_{3}\right)$

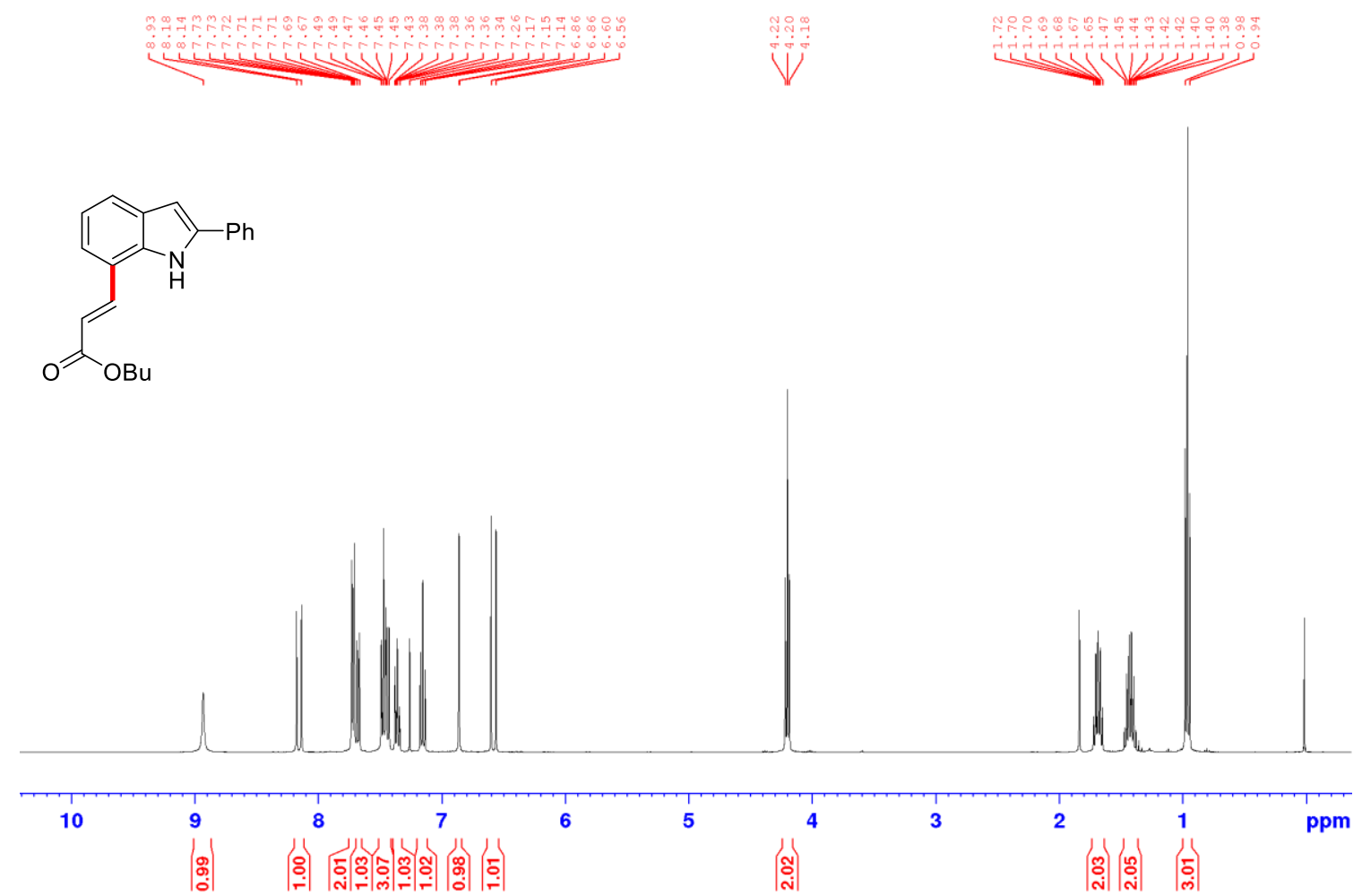

${ }^{13} \mathrm{C} \mathrm{NMR}$ of 3 ma $\left(100 \mathrm{MHz}, \mathrm{CDCl}_{3}\right)$

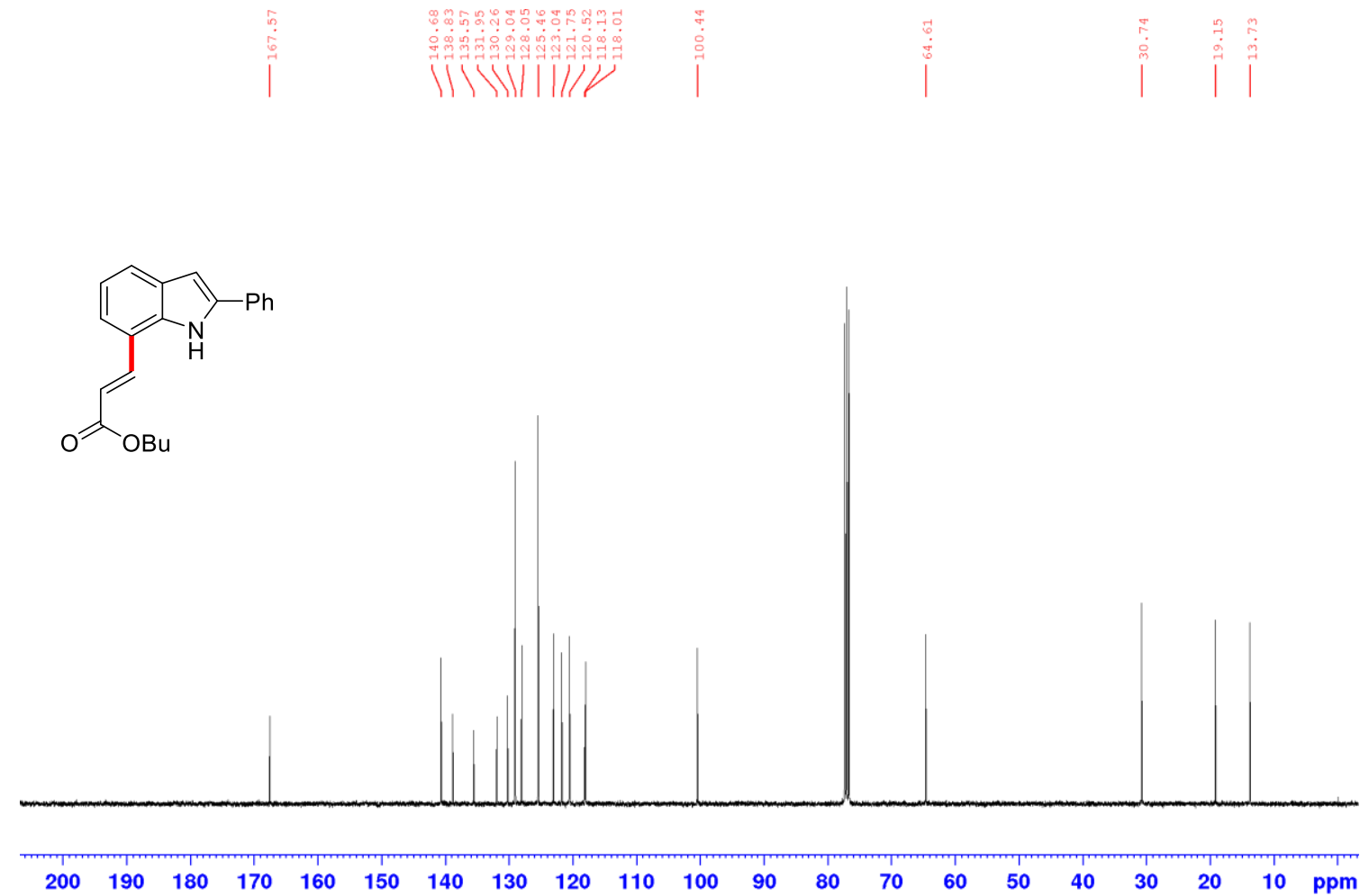


${ }^{1} \mathrm{H}$ NMR of 3na (400 MHz, $\left.\mathrm{CDCl}_{3}\right)$

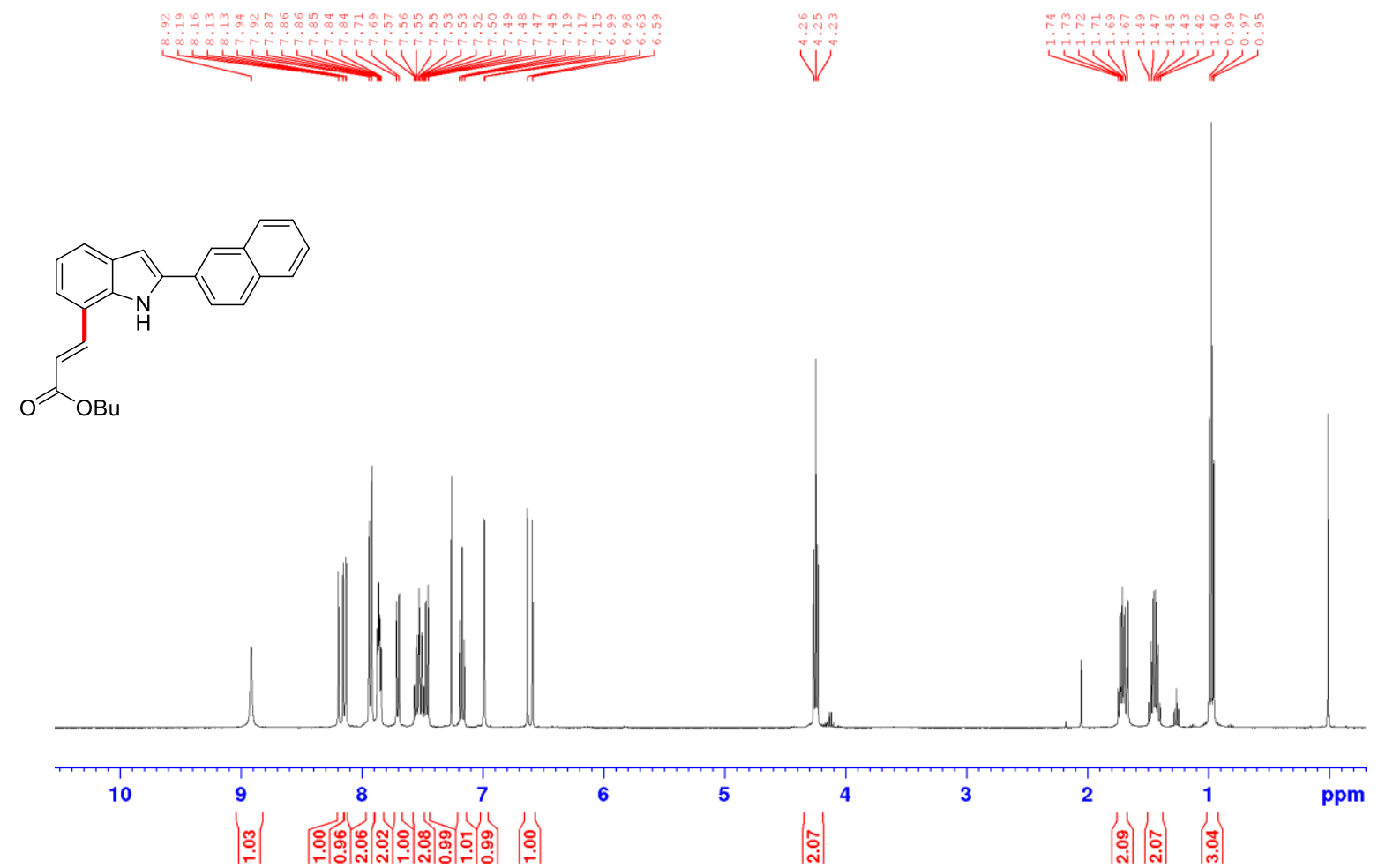

${ }^{13} \mathrm{C} \mathrm{NMR}$ of 3na $\left(100 \mathrm{MHz}, \mathrm{CDCl}_{3}\right)$
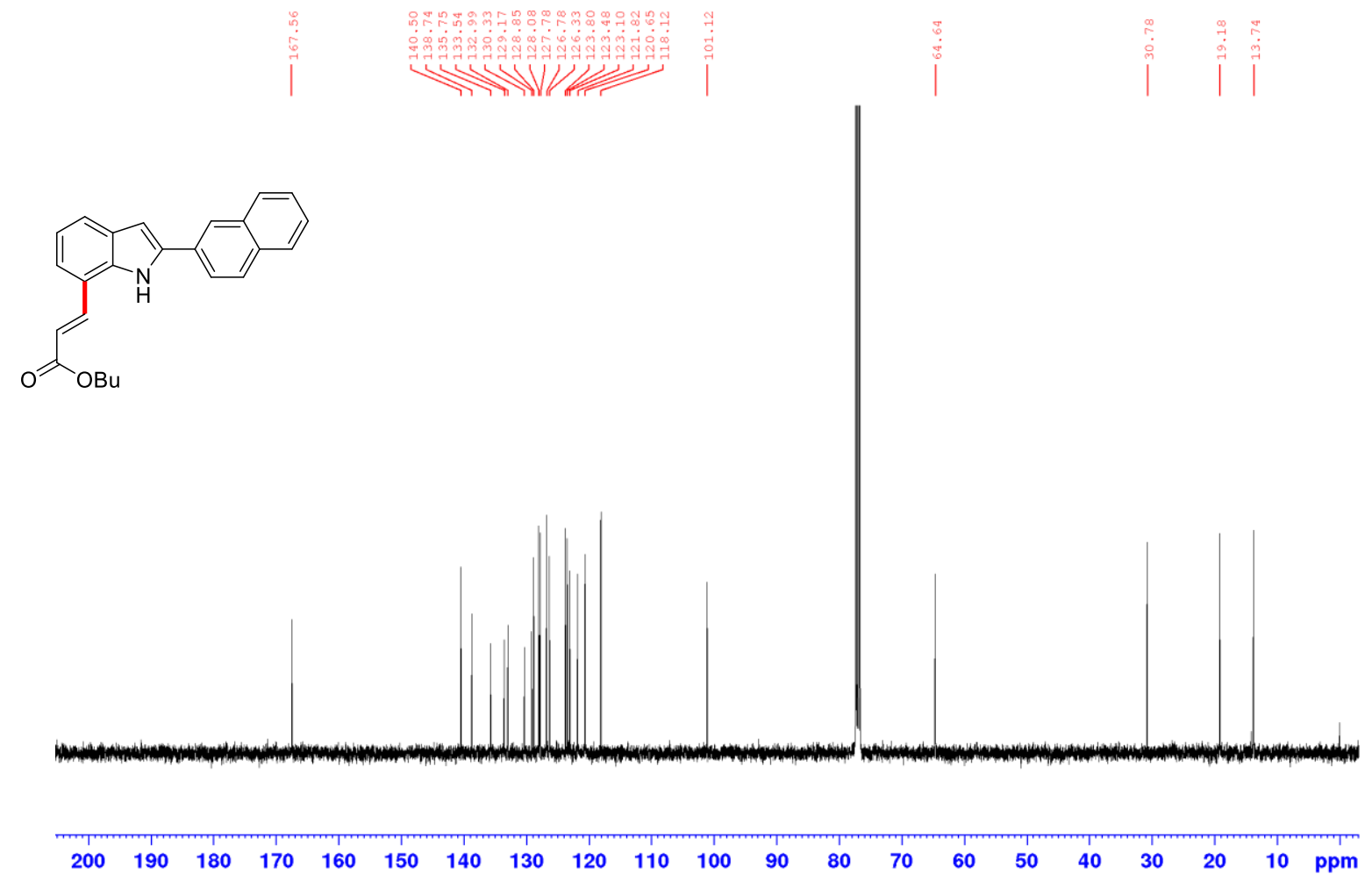
${ }^{1} \mathrm{H}$ NMR of $3 \mathbf{o a}\left(400 \mathrm{MHz}, \mathrm{CDCl}_{3}\right)$

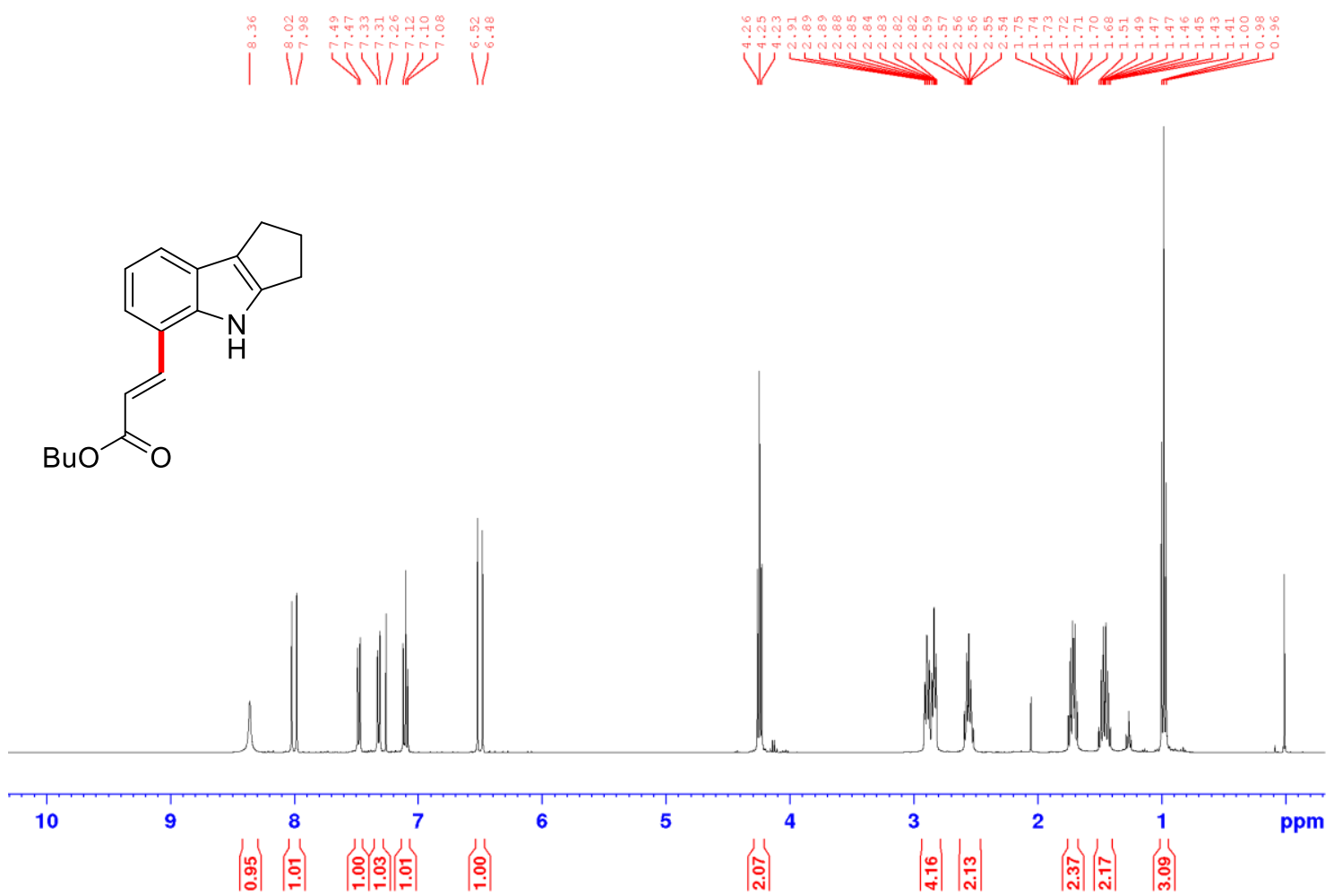

${ }^{13} \mathrm{C}$ NMR of $3 \mathbf{o a}\left(100 \mathrm{MHz}, \mathrm{CDCl}_{3}\right)$

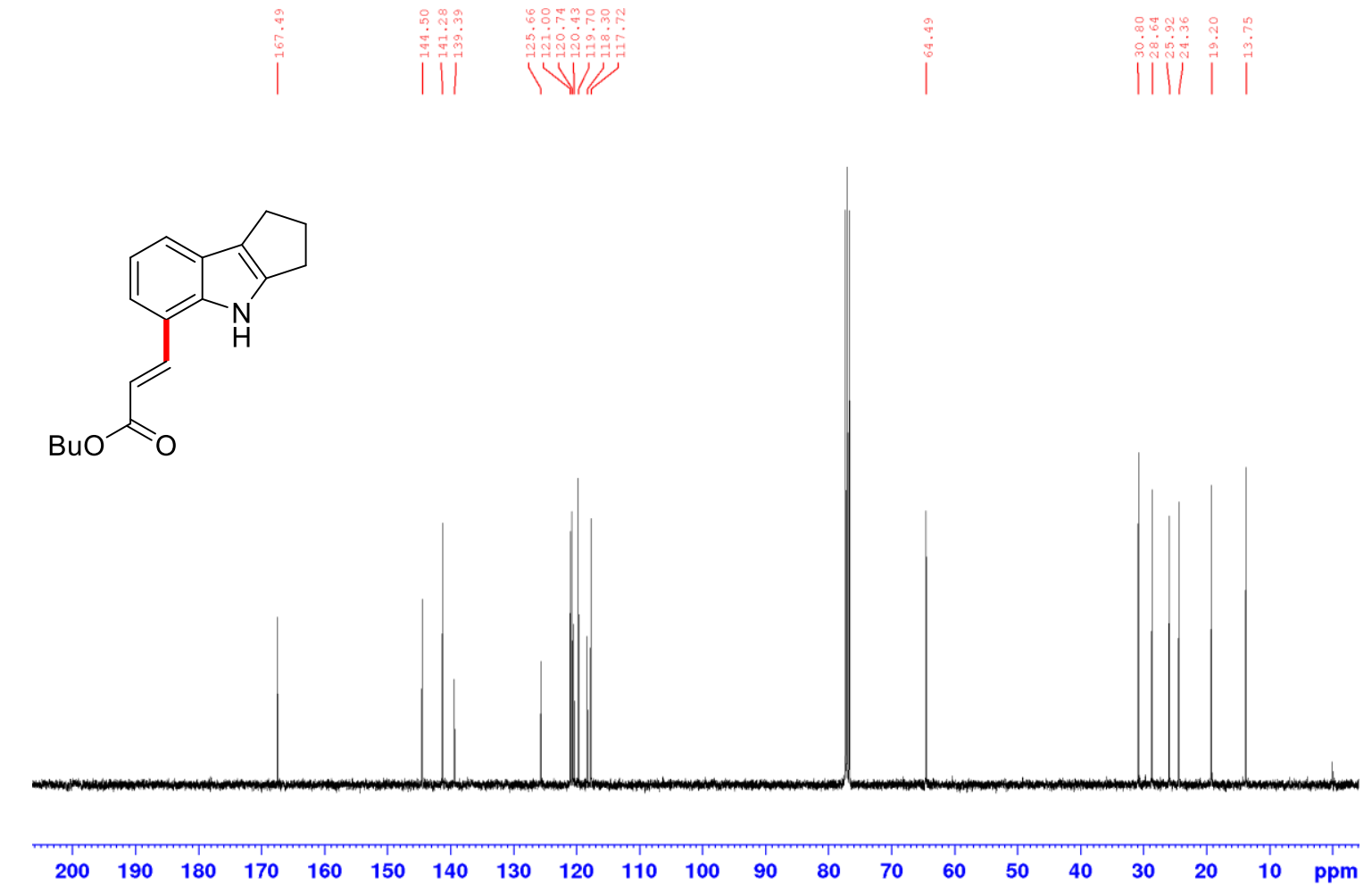


${ }^{1} \mathrm{H}$ NMR of 3pa (400 MHz, $\left.\mathrm{CDCl}_{3}\right)$
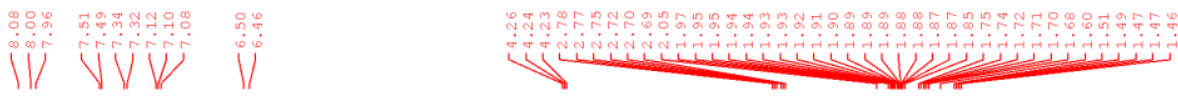<smiles>CC(C)(C)OC(=O)/C=C/c1cccc2c3c([nH]c12)CCCC3</smiles>

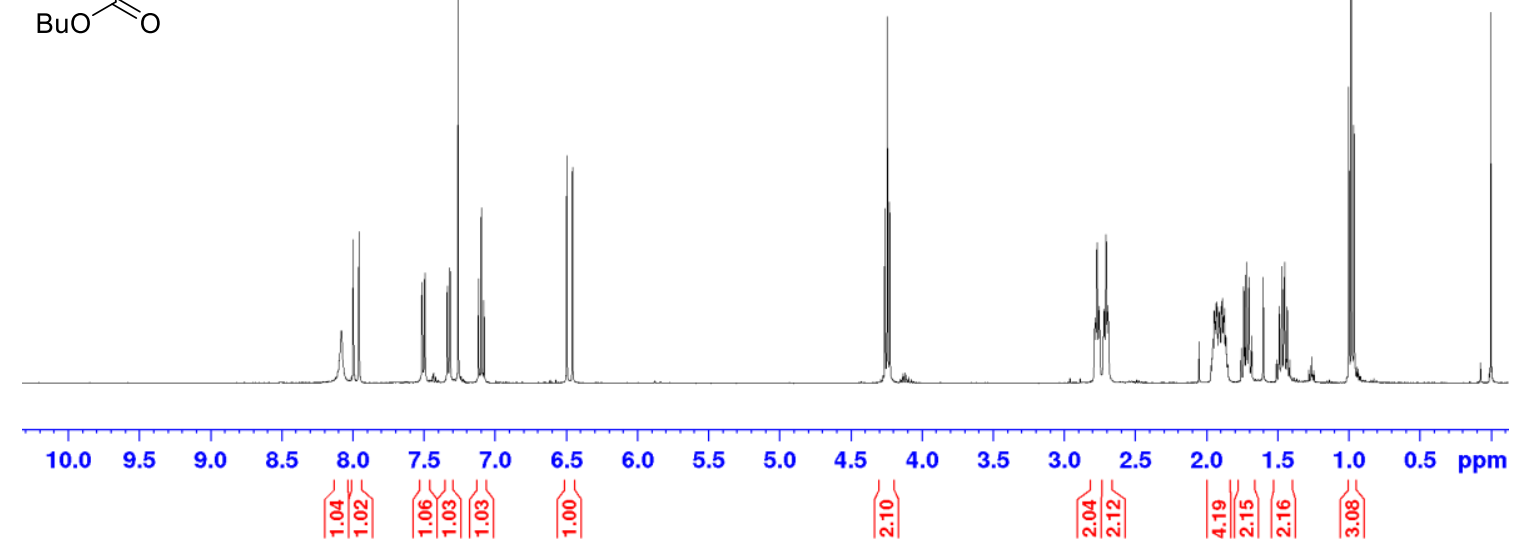

${ }^{13} \mathrm{C}$ NMR of 3pa $\left(100 \mathrm{MHz}, \mathrm{CDCl}_{3}\right)$

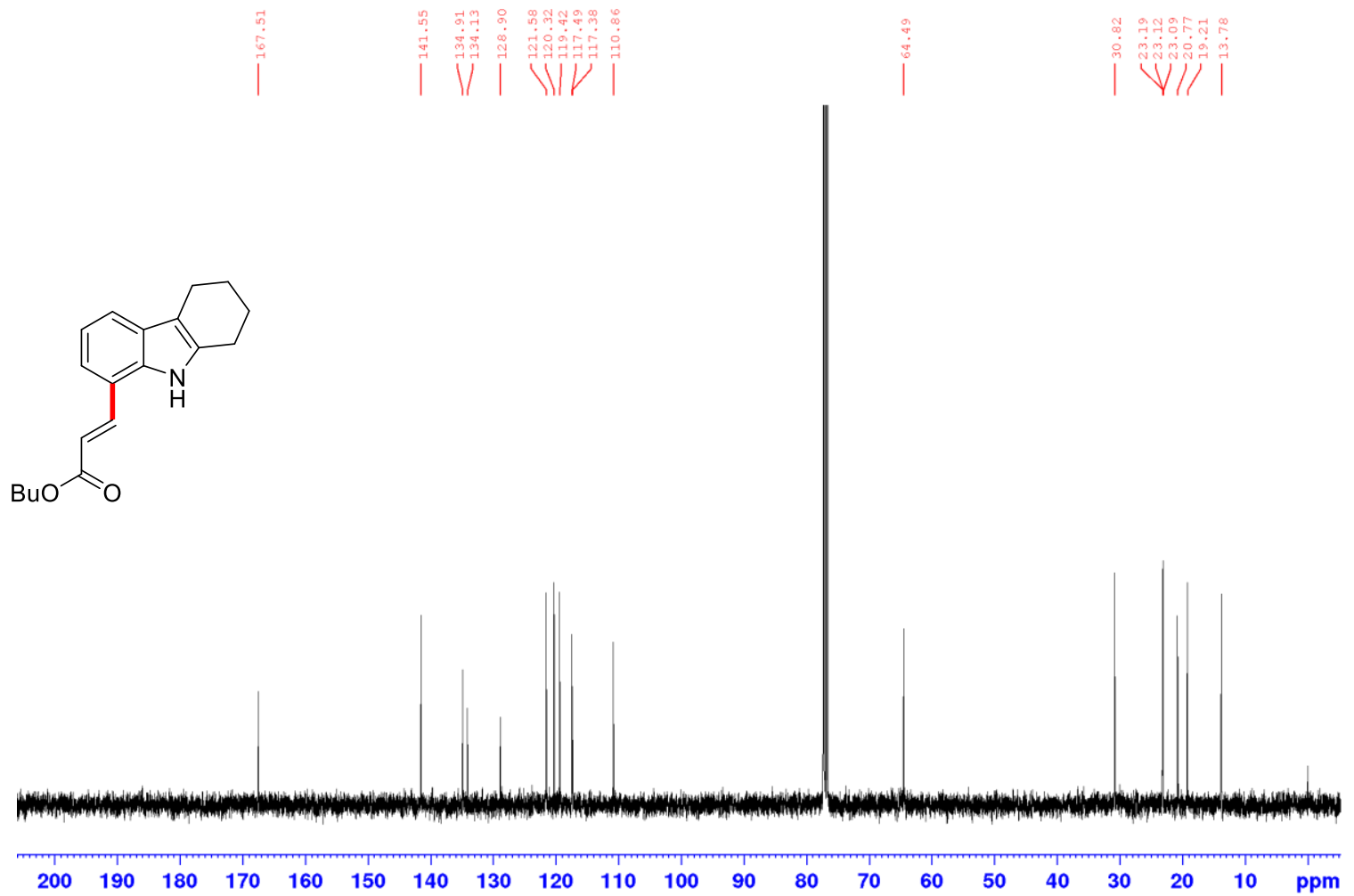


${ }^{1} \mathrm{H}$ NMR of $\mathbf{3 q a}\left(400 \mathrm{MHz}, \mathrm{CDCl}_{3}\right)$

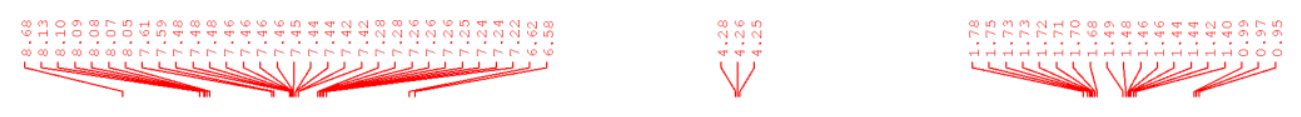<smiles>CC(C)(C)OC(=O)/C=C/c1cccc2c1[nH]c1ccccc12</smiles>

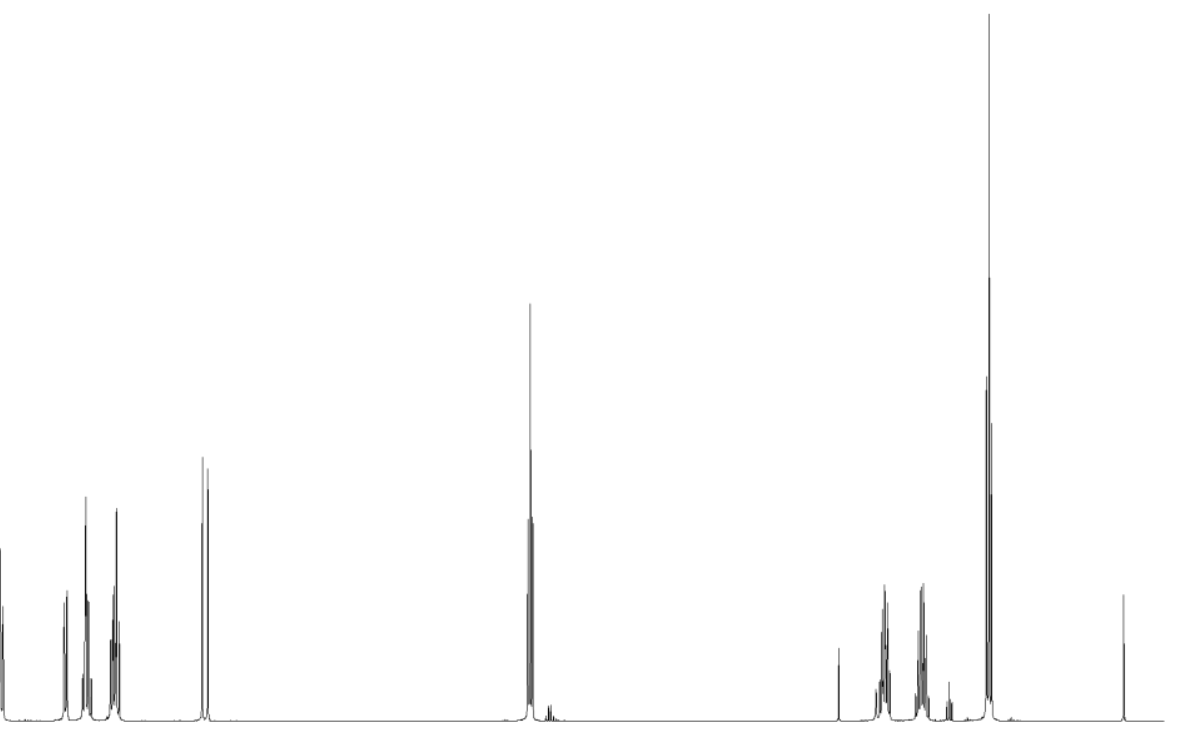

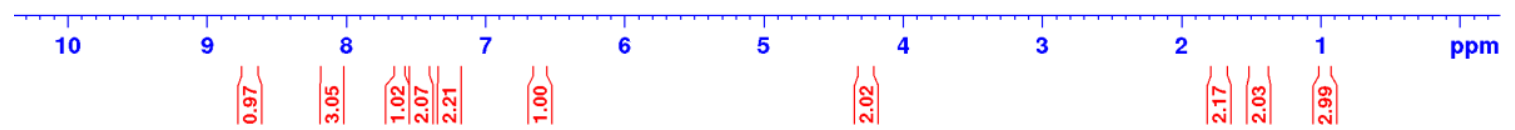

${ }^{13} \mathrm{C} \mathrm{NMR}$ of 3qa $\left(100 \mathrm{MHz}, \mathrm{CDCl}_{3}\right)$<smiles>CC(C)(C)OC(=O)/C=C/c1cccc2c1[nH]c1ccccc12</smiles>

$$
\text { BuO }
$$

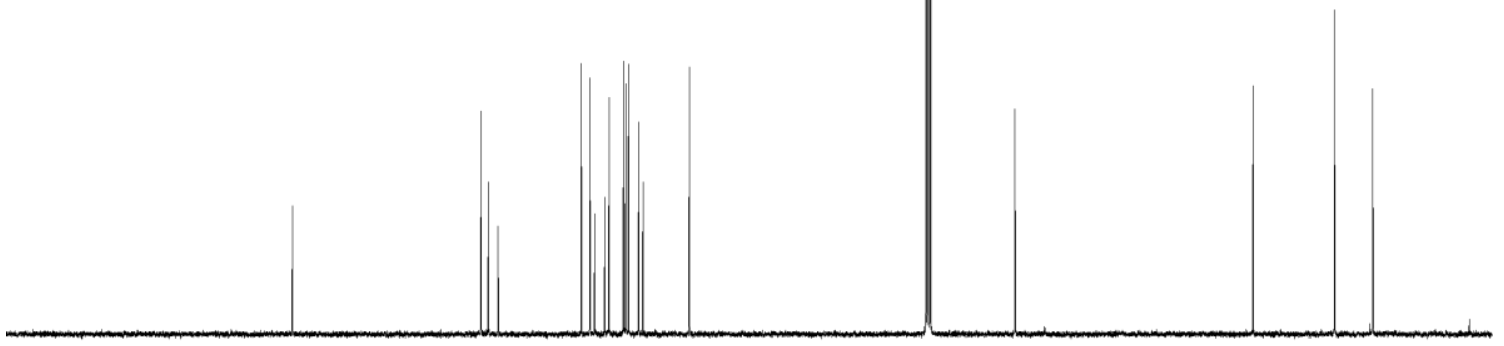

$\begin{array}{lllllllllllllllllllllllll}200 & 190 & 180 & 170 & 160 & 150 & 140 & 130 & 120 & 110 & 100 & 90 & 80 & 70 & 60 & 50 & 40 & 30 & 20 & 10 & \text { ppm }\end{array}$ 
${ }^{1} \mathrm{H} \mathrm{NMR}$ of $\mathbf{4 j a}\left(400 \mathrm{MHz}, \mathrm{CDCl}_{3}\right)$

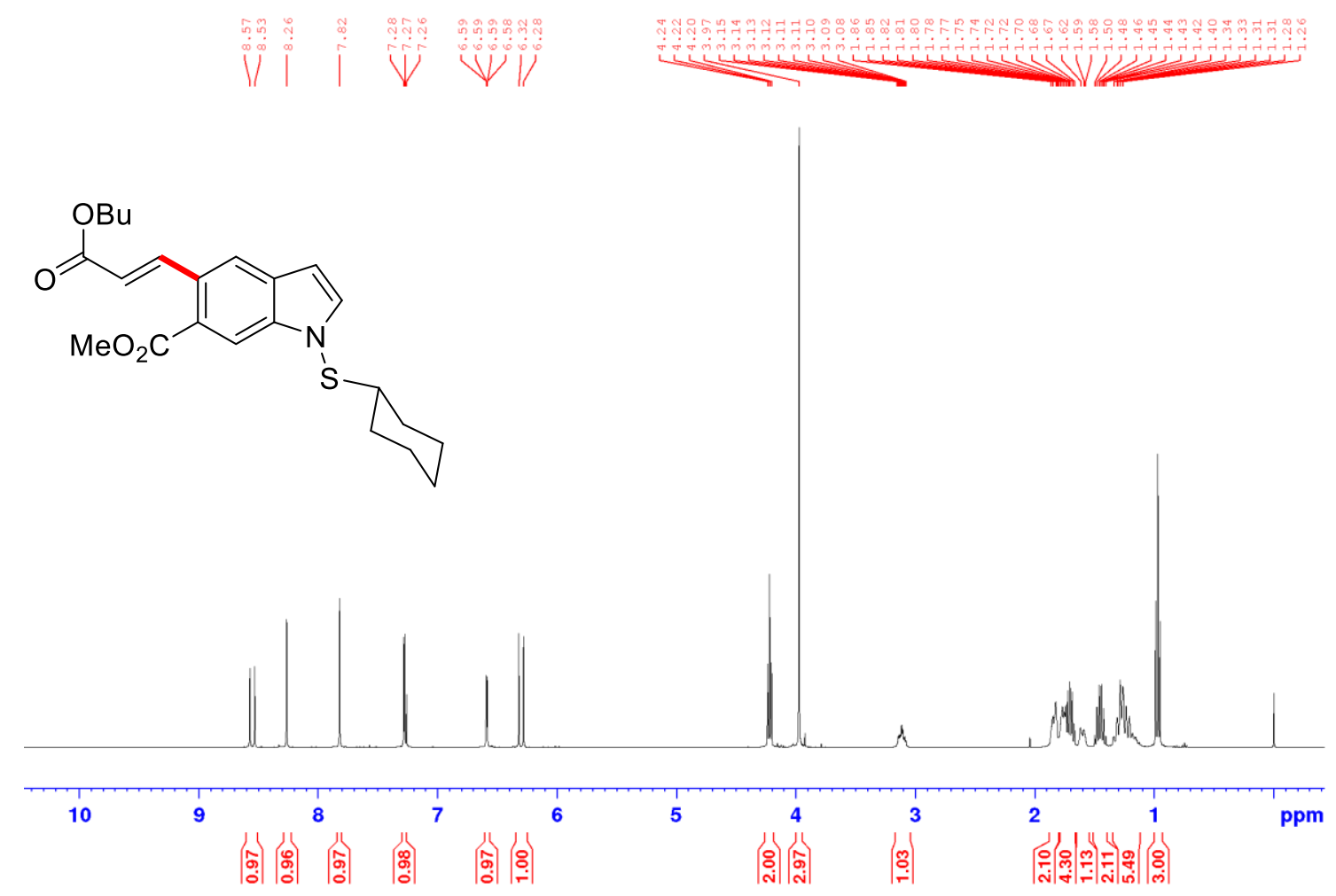

${ }^{13} \mathrm{C}$ NMR of $\mathbf{4 j a}\left(100 \mathrm{MHz}, \mathrm{CDCl}_{3}\right)$

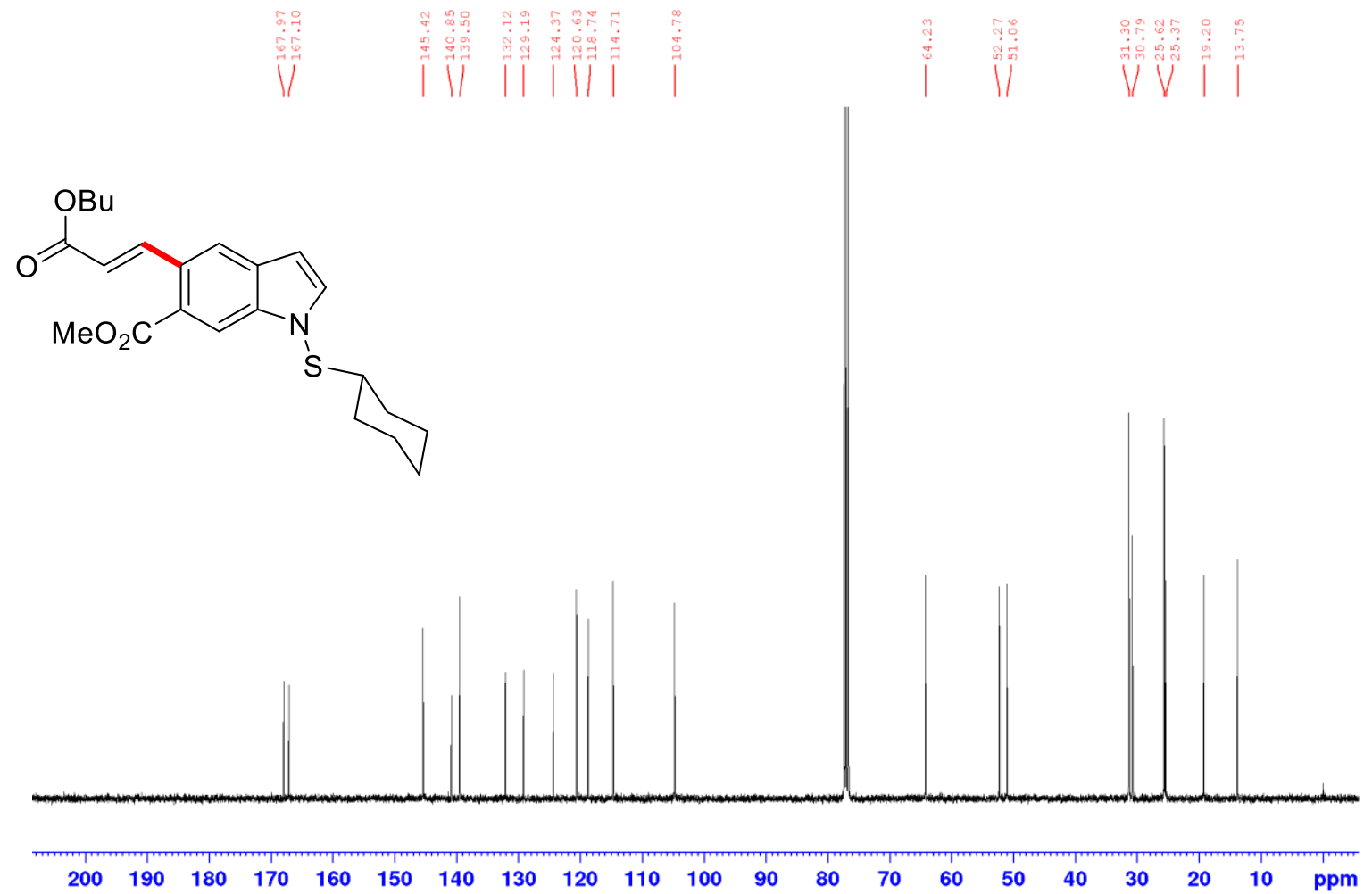


${ }^{1} \mathrm{H}$ NMR of 3ba-1 (400 MHz, $\mathrm{CDCl}_{3}$ )

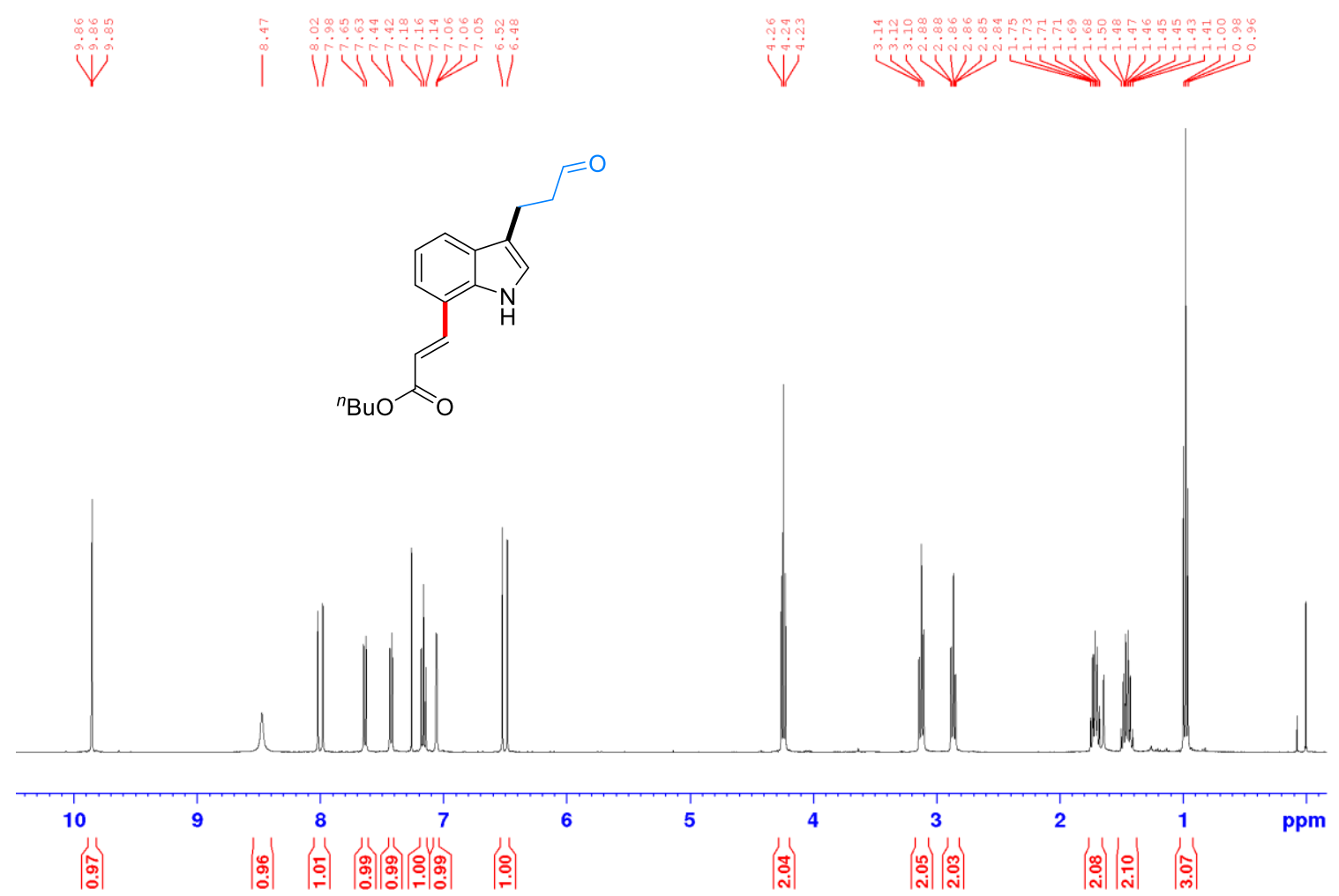

${ }^{13} \mathrm{C}$ NMR of 3ba-1 $\left(100 \mathrm{MHz}, \mathrm{CDCl}_{3}\right)$

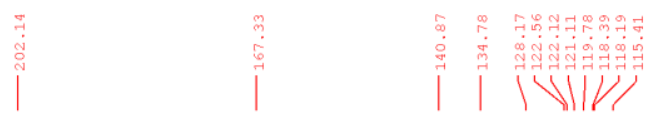<smiles>CCCCOC(=O)/C=C/c1cccc2c(CCC=O)c[nH]c12</smiles>
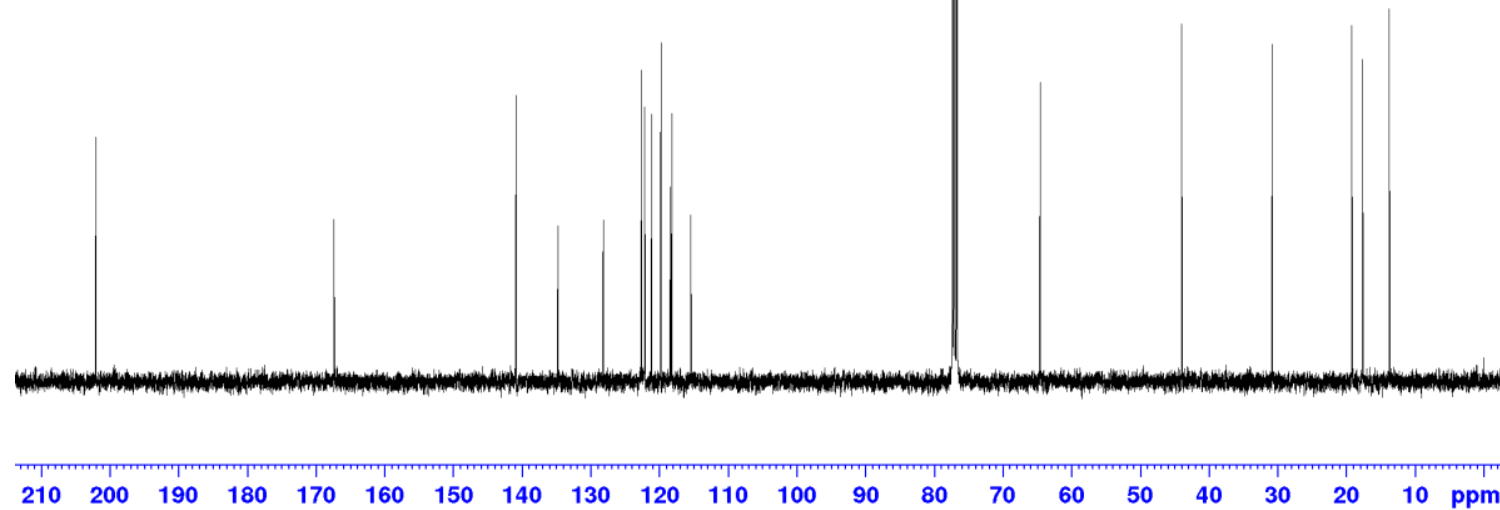
${ }^{1} \mathrm{H}$ NMR of 3ba-2 (400 MHz, $\mathrm{CDCl}_{3}$ )

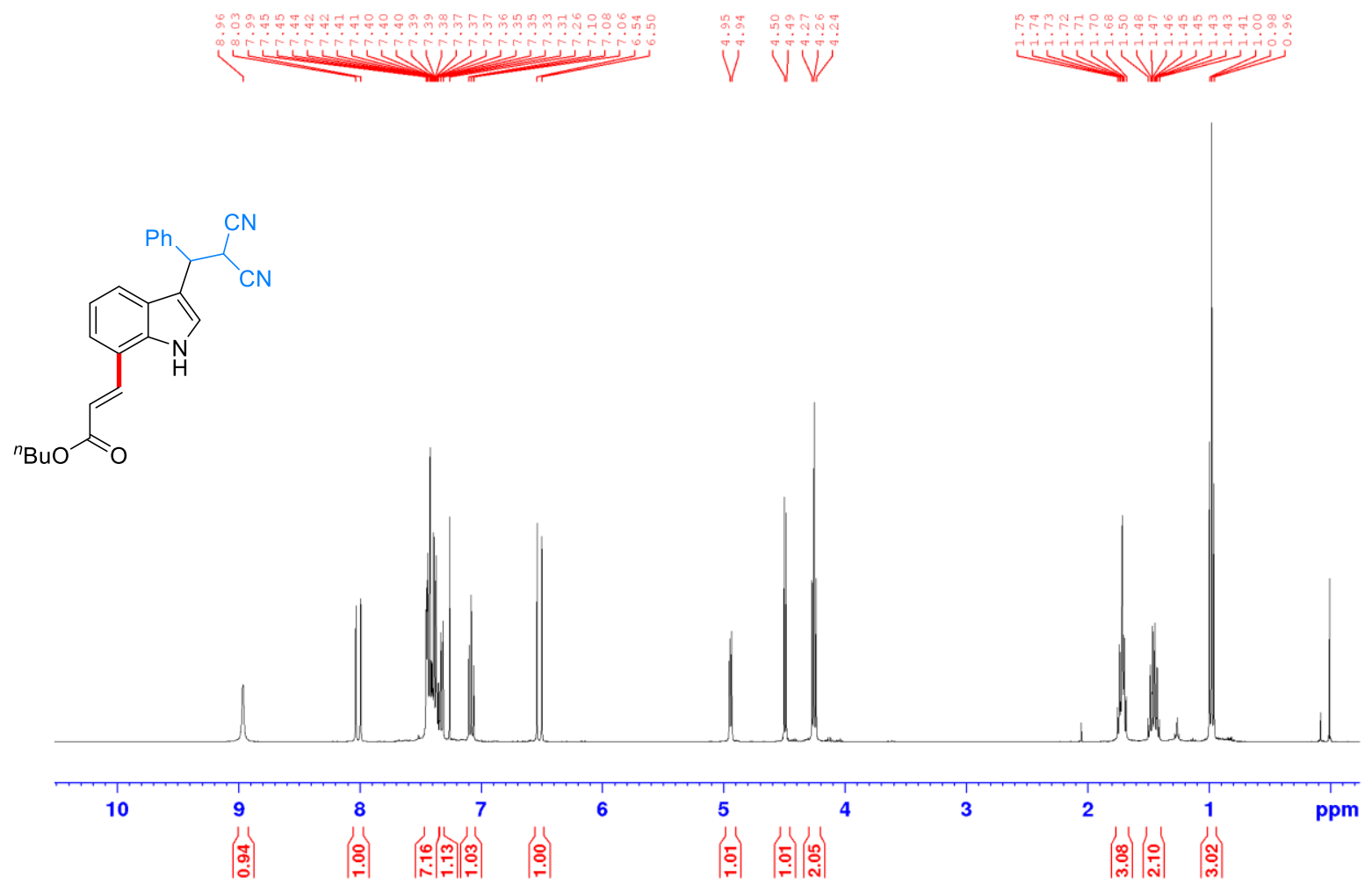

${ }^{13} \mathrm{C}$ NMR of $\mathbf{3 b a}-2\left(100 \mathrm{MHz}, \mathrm{CDCl}_{3}\right)$

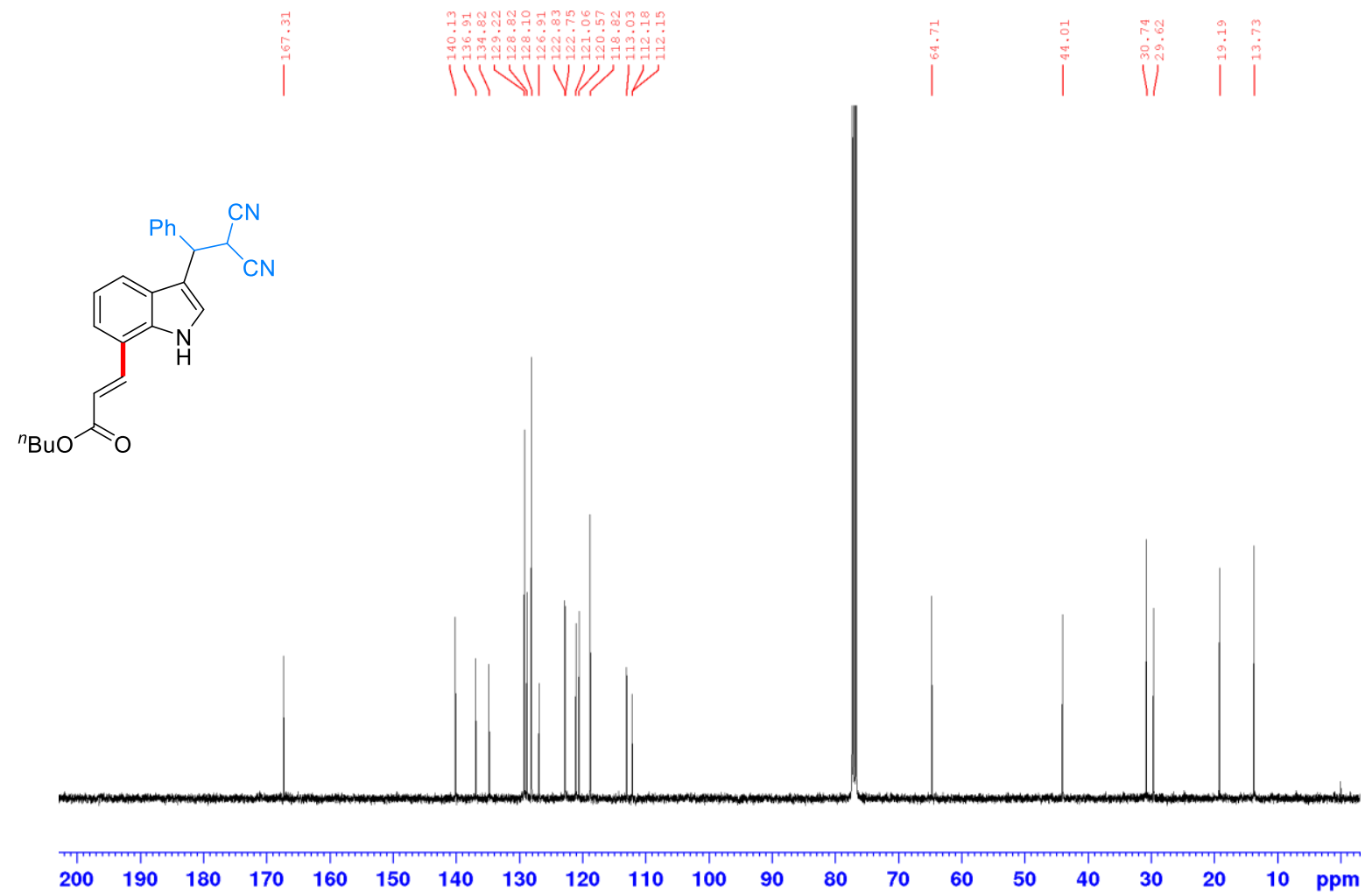


${ }^{1} \mathrm{H}$ NMR of 3ka-1 (400 MHz, $\left.\mathrm{CDCl}_{3}\right)$

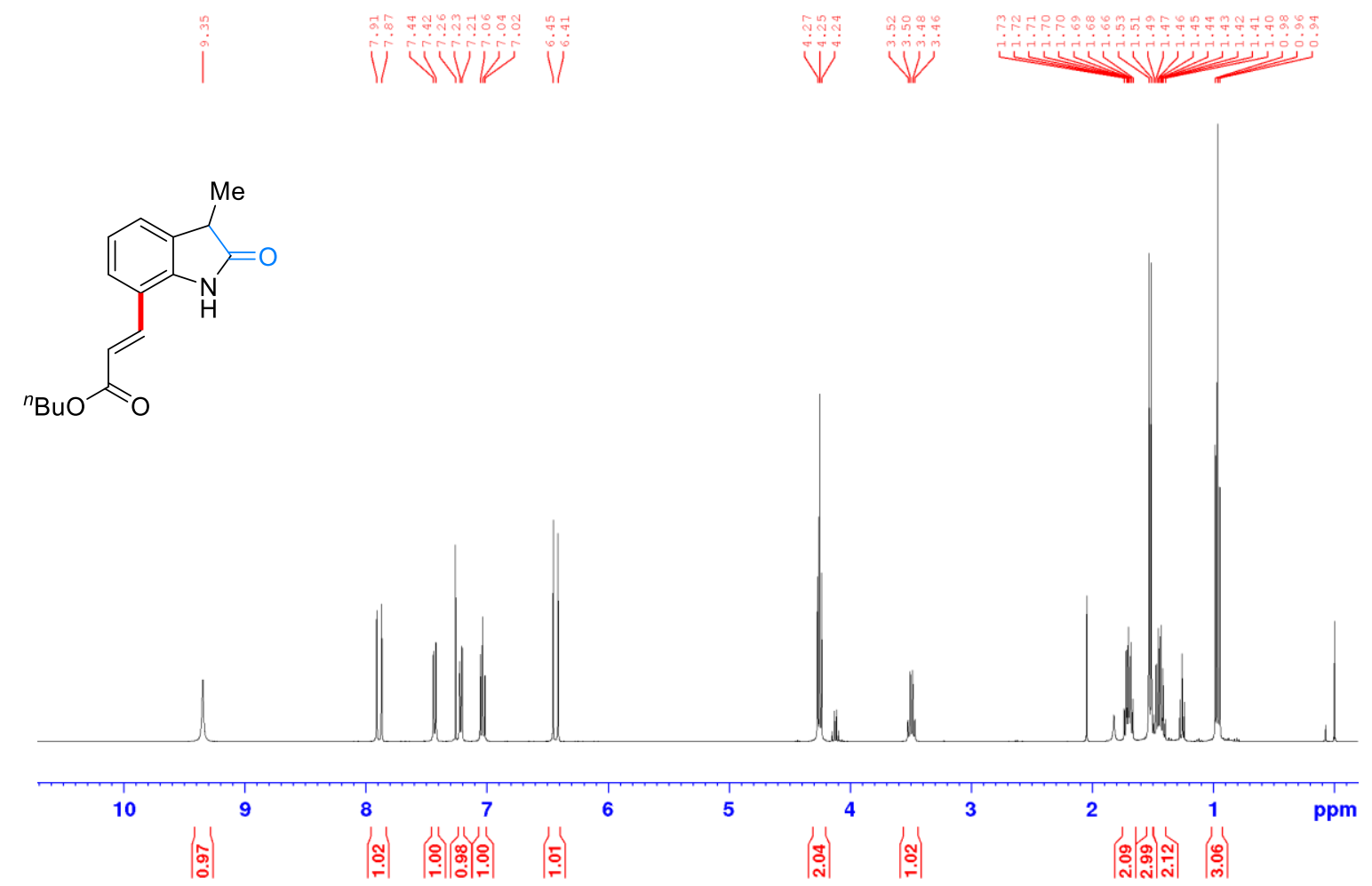

${ }^{13} \mathrm{C}$ NMR of 3ka-1 $\left(100 \mathrm{MHz}, \mathrm{CDCl}_{3}\right)$

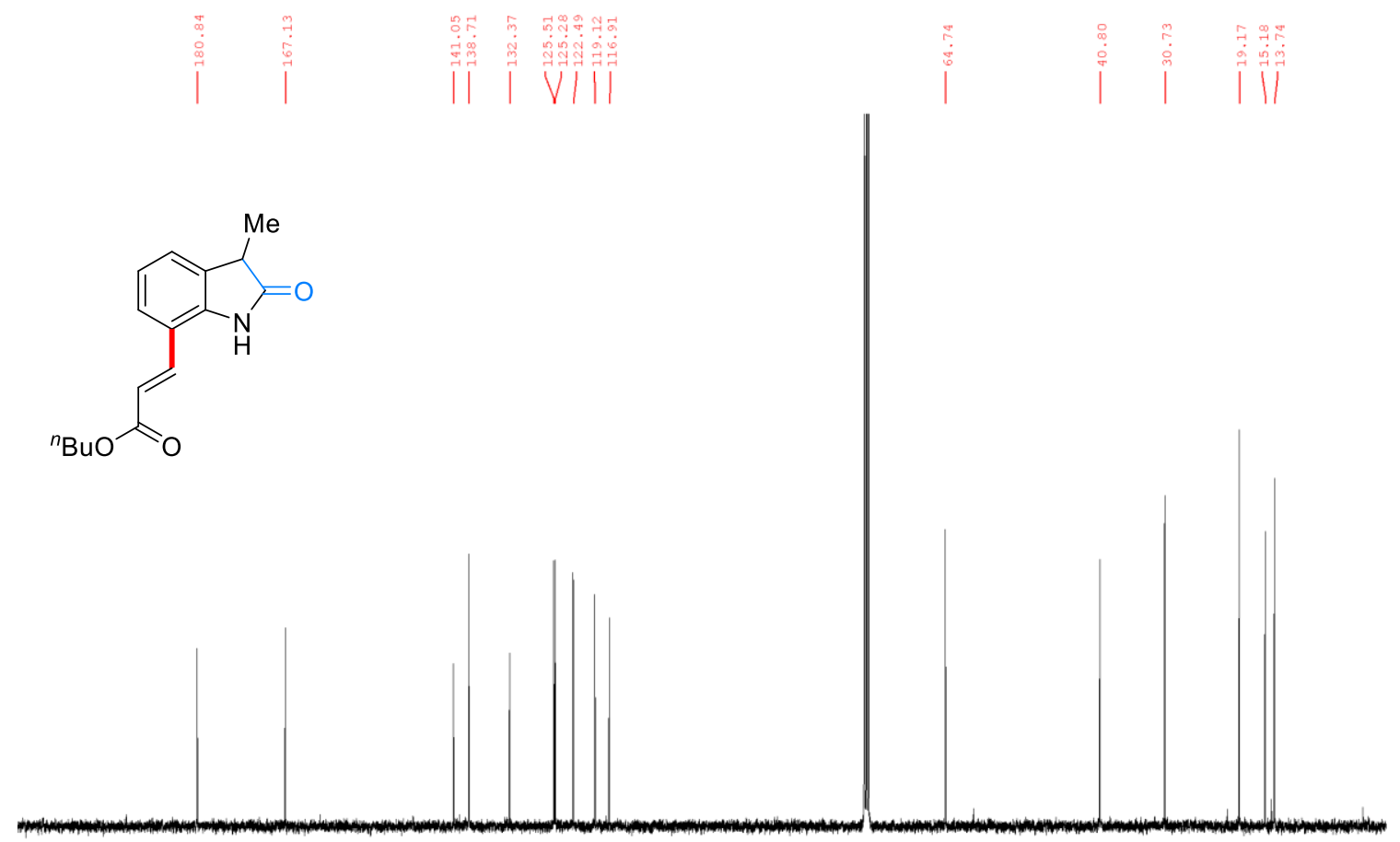

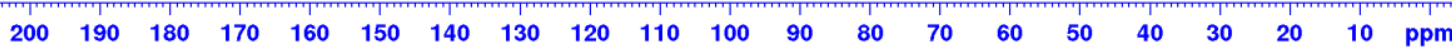


${ }^{1} \mathrm{H}$ NMR of 3ba-4 $\left(400 \mathrm{MHz}, \mathrm{CDCl}_{3}\right)$

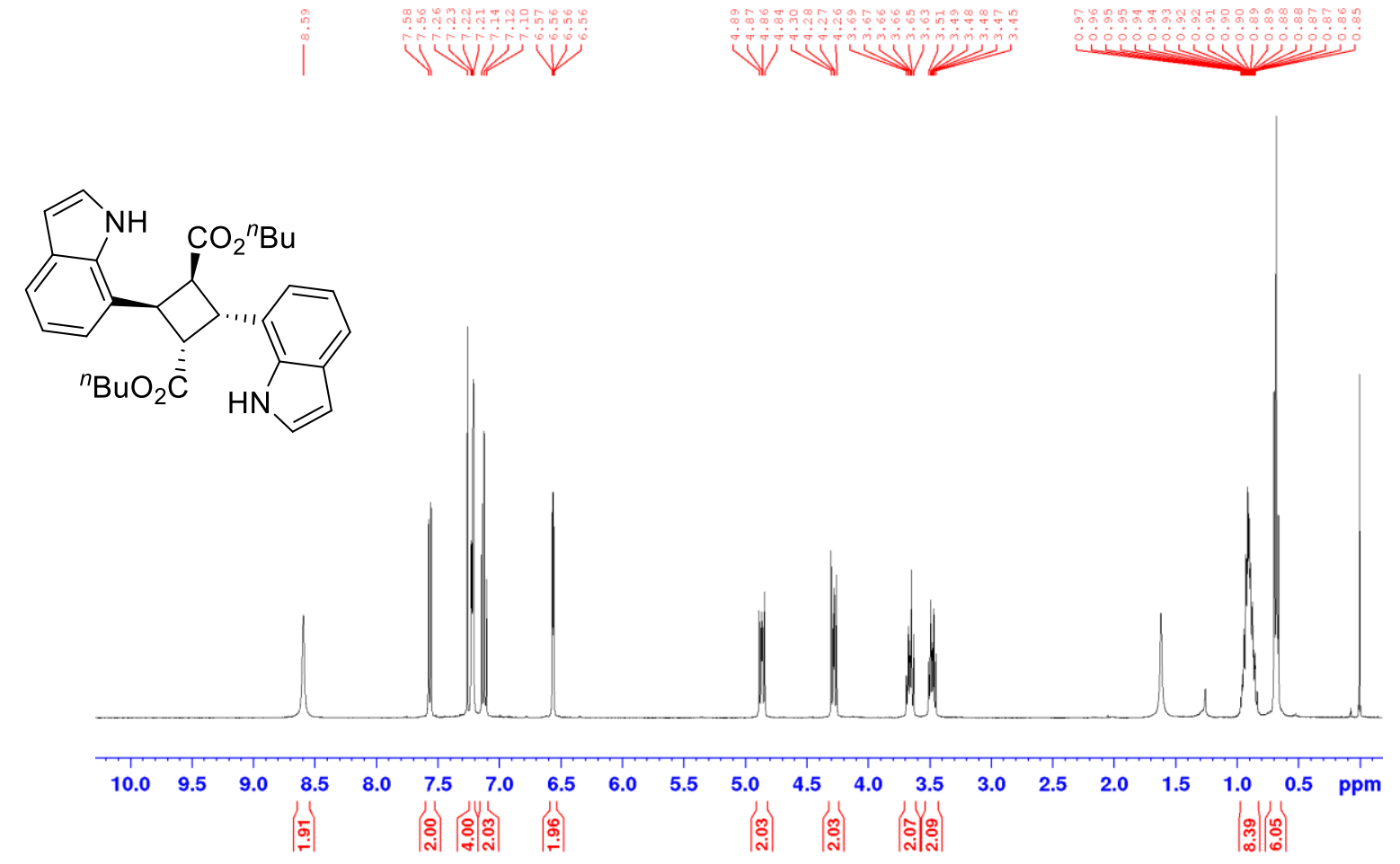

${ }^{13} \mathrm{C}$ NMR of 3ba-4 (100 MHz, $\left.\mathrm{CDCl}_{3}\right)$<smiles>CCC(C)OC(=O)[C@H]1[C@@H](c2cccc3cc[nH]c23)[C@H](c2cccc3cc[nH]c23)[C@H]1C(=O)OCC(C)(C)C</smiles>
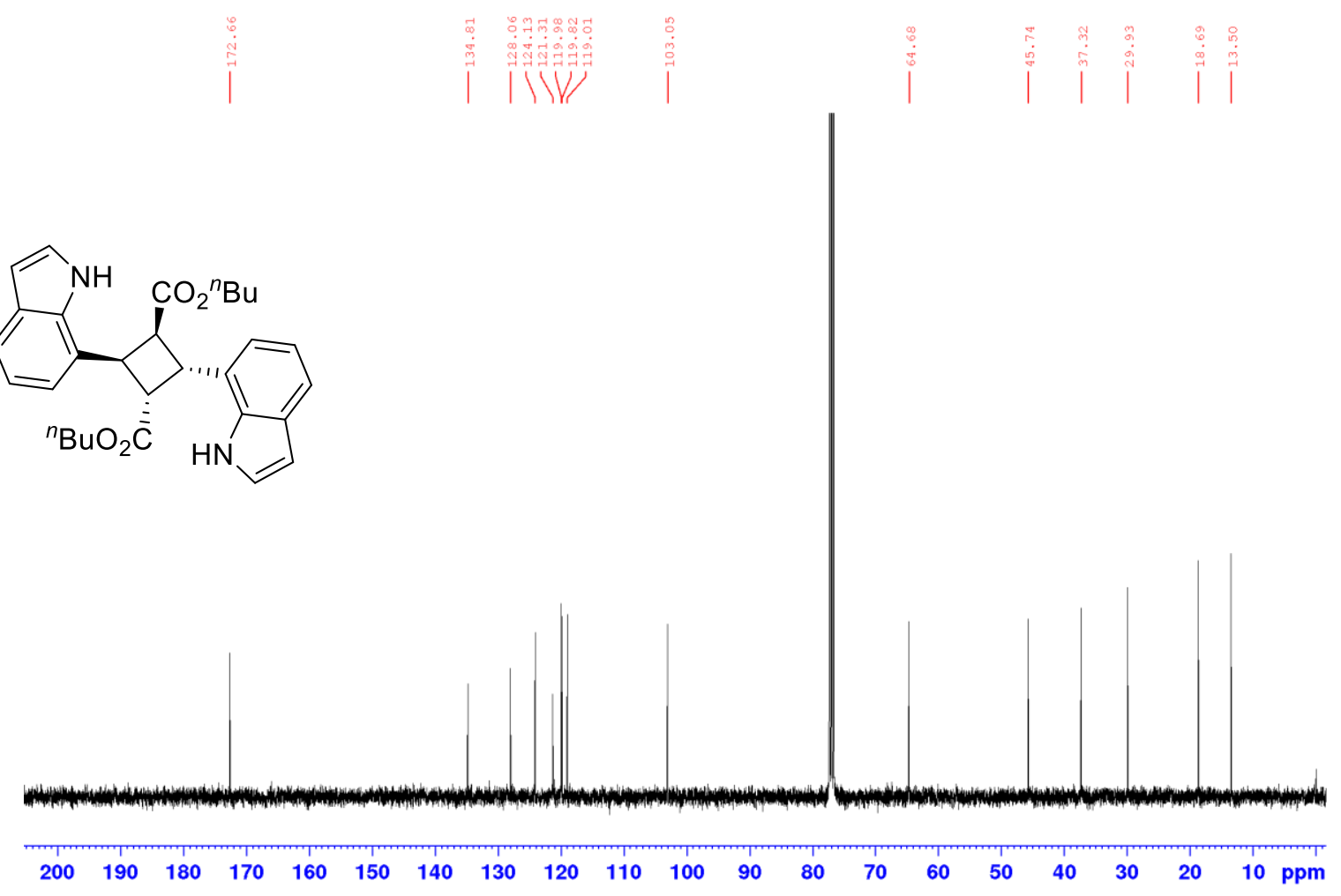
${ }^{1} \mathrm{H}$ NMR of 3ba-5 (400 MHz, $\left.\mathrm{CDCl}_{3}\right)$

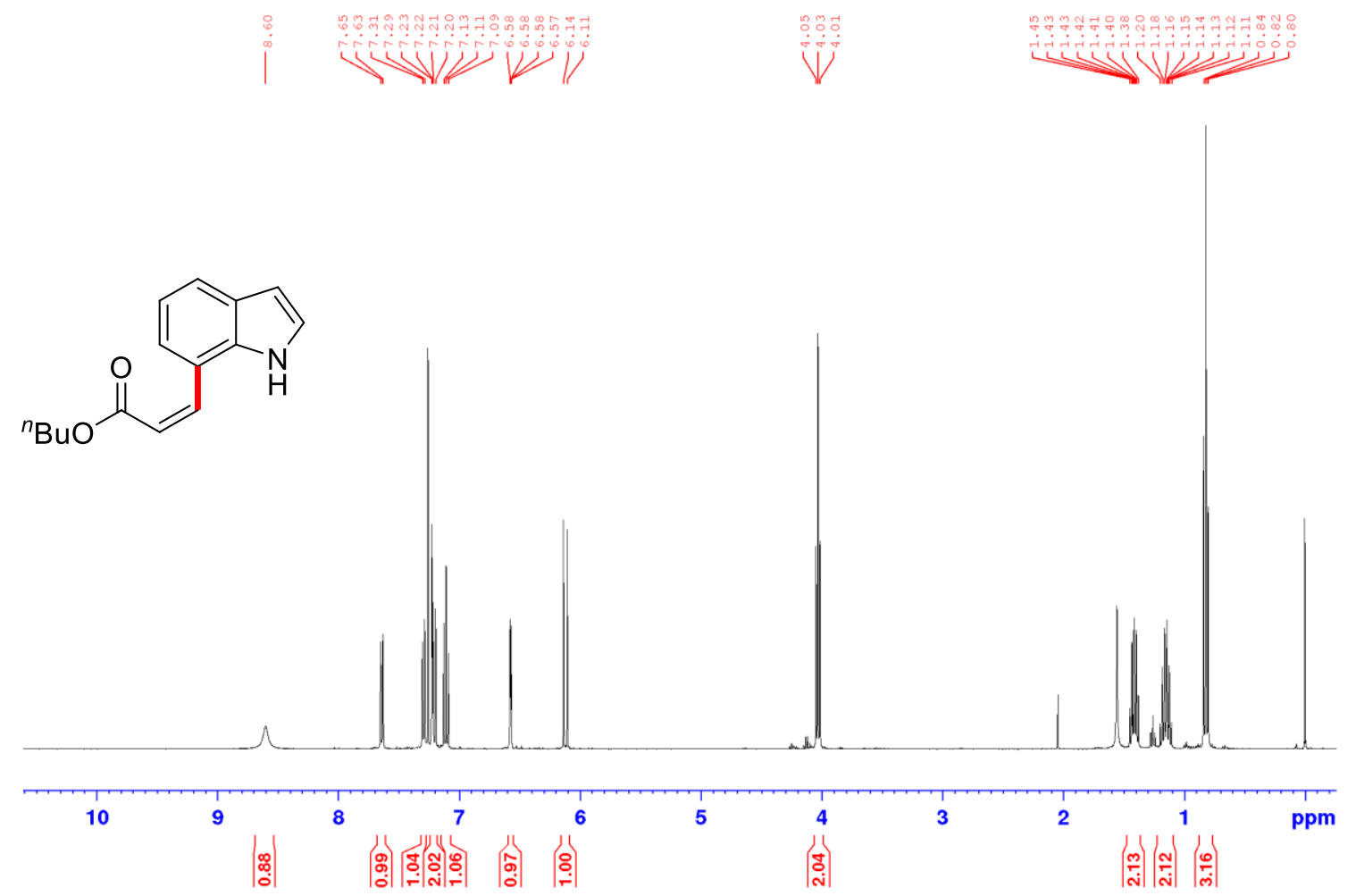

${ }^{13} \mathrm{C}$ NMR of $\mathbf{3 b a}-5\left(100 \mathrm{MHz}, \mathrm{CDCl}_{3}\right)$

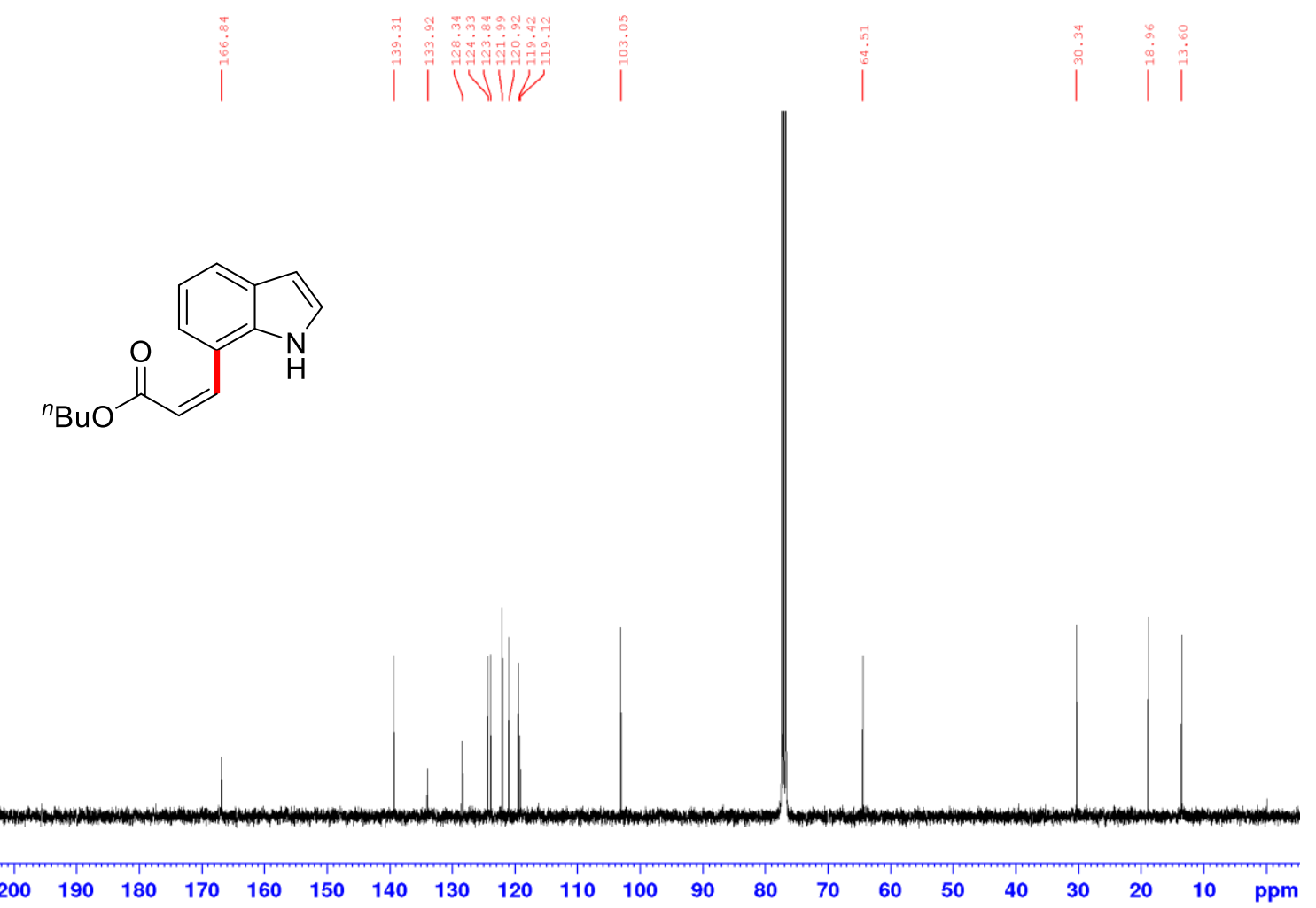




\section{References}

${ }^{1}$ Barday, M.; Janot, C.; Halcovitch, N. R.; Muir, J.; Aïssa, C. Angew. Chem., Int. Ed. 2017, 56, 13117-13121.

${ }^{2}$ Xu, L.; Tan, L.; Ma, D. Synlett 2017, 28, 2839-2844.

${ }^{3}$ Yang, D.; Mao, S.; Gao, Y.-R.; Guo, D.-D.; Guo, S.-H.; Li, B.; Wang, Y.-Q. RSC Advances 2015, 5, $23727-$ 23736 .

${ }^{4}$ Urones, B.; Arrayás, R. G.; Carretero, J. C. Pd ${ }^{\mathrm{II}}$-Catalyzed Di-o-olefination of Carbazoles Directed by the Protecting N-(2-Pyridyl)sulfonyl Group. Org. Lett. 2013, 15, 1120-1123.

${ }^{5}$ Sheldrick, G. M. A short history of SHELX. Acta Crystallogr. Sect. A 2008, 64, 112-122.

${ }^{6}$ Farrugia, L. J. WinGX and ORTEP for Windows: an update. J. Appl. Cryst. 2012, 45, 849-854.

${ }^{7}$ Frisch, M. J.; Trucks, G. W.; Schlegel, H. B.; Scuseria, G. E.; Robb, M. A.; Cheeseman, J. R.; Scalmani, G.; Barone, V.; Petersson, G. A.; Nakatsuji, H.; Li, X.; Caricato, M.; Marenich, A. V.; Bloino, J.; Janesko, B. G.; Gomperts, R.; Mennucci, B.; Hratchian, H. P.; Ortiz, J. V.; Izmaylov, A. F.; Sonnenberg, J. L.; Williams-Young, D.; Ding, F.; Lipparini, F.; Egidi, F.; Goings, J.; Peng, B.; Petrone, A.; Henderson, T.; Ranasinghe, D.; Zakrzewski, V. G.; Gao, J.; Rega, N.; Zheng, G.; Liang, W.; Hada, M.; Ehara, M.; Toyota, K.; Fukuda, R.; Hasegawa, J.; Ishida, M.; Nakajima, T.; Honda, Y.; Kitao, O.; Nakai, H.; Vreven, T.; Throssell, K.; Montgomery, J. A., Jr.; Peralta, J. E.; Ogliaro, F.; Bearpark, M. J.; Heyd, J. J.; Brothers, E. N.; Kudin, K. N.; Staroverov, V. N.; Keith, T. A.; Kobayashi, R.; Normand, J.; Raghavachari, K.; Rendell, A. P.; Burant, J. C.; Iyengar, S. S.; Tomasi, J.; Cossi, M.; Millam, J. M.; Klene, M.; Adamo, C.; Cammi, R.; Ochterski, J. W.; Martin, R. L.; Morokuma, K.; Farkas, O.; Foresman, J. B.; Fox, D. J. Gaussian, Inc., Wallingford CT, 2016. 